\section{Pacific Northwest}

National Laboratory

Operated by Battelle for the

U.S. Department of Energy

\title{
Testing of Large-Scale ICV Glasses with Hanford LAW Simulant
}

\author{
P. R. Hrma \\ D.-S. Kim \\ J. D. Vienna \\ J. Matyáš \\ D. E. Smith \\ M. J. Schweiger \\ J. D. Yeager
}

March 2005

Prepared for the U.S. Department of Energy under Contract DE-AC05-76RL01830 


\title{
DISCLAIMER
}

This report was prepared as an account of work sponsored by an agency of the United States Government. Neither the United States Government nor any agency thereof, nor Battelle Memorial Institute, nor any of their employees, makes any warranty, express or implied, or assumes any legal liability or responsibility for the accuracy, completeness, or usefulness of any information, apparatus, product, or process disclosed, or represents that its use would not infringe privately owned rights. Reference herein to any specific commercial product, process, or service by trade name, trademark, manufacturer, or otherwise does not necessarily constitute or imply its endorsement, recommendation, or favoring by the United States Government or any agency thereof, or Battelle Memorial Institute. The views and opinions of authors expressed herein do not necessarily state or reflect those of the United States Government or any agency thereof.

\author{
PACIFIC NORTHWEST NATIONAL LABORATORY \\ operated by \\ BATTELLE \\ for the \\ UNITED STATES DEPARTMENT OF ENERGY \\ under Contract DE-AC06-76RL01830
}

This document was printed on recycled paper. 


\title{
Testing of Large-Scale ICV Glasses with Hanford LAW Simulant
}

\author{
P. R. Hrma \\ D.-S. Kim \\ J. D. Vienna \\ J. Matyáš \\ D. E. Smith \\ M. J. Schweiger \\ J. D. Yeager
}

March 2005

Prepared for the U.S. Department of Energy

under Contract DE-AC06-76RL01830

Pacific Northwest National Laboratory

Richland, WA 99352 


\section{Summary}

Preliminary glass compositions for immobilizing Hanford low-activity waste (LAW) by the in-container vitrification (ICV) process were initially fabricated at crucible- and engineering-scale, including simulants and actual (radioactive) LAW. Glasses were characterized for vapor hydration test (VHT) and product consistency test (PCT) responses and crystallinity (both quenched and slow-cooled samples). Selected glasses were tested for toxicity characteristic leach procedure (TCLP) responses, viscosity, and electrical conductivity. This testing showed that glasses with LAW loading of 20 mass $\%$ can be made readily and meet all product constraints by a far margin. Glasses with over 22 mass $\% \mathrm{Na}_{2} \mathrm{O}$ can be made to meet all other product quality and process constraints.

Large-scale testing was performed at the AMEC, Geomelt Division facility in Richland. Three tests were conducted using simulated LAW with increasing loadings of 12,17 , and 20 mass $\% \mathrm{Na}_{2} \mathrm{O}$. Glass samples were taken from the test products in a manner to represent the full expected range of product

performance. These samples were characterized for composition, density, crystalline and non-crystalline phase assemblage, and durability using the VHT, PCT, and TCLP tests. The results, presented in this report, show that the AMEC ICV product with meets all waste form requirements with a large margin. These results provide strong evidence that the Hanford LAW can be successfully vitrified by the ICV technology and can meet all the constraints related to product quality. The economic feasibility of the ICV technology can be further enhanced by subsequent optimization. 



\section{Abbreviations and Acronyms}

\begin{tabular}{|c|c|}
\hline AES & atomic emission spectroscopy \\
\hline AMBG & AMEC bulk vitrification glass \\
\hline AMOG & AMEC oxidation state glass \\
\hline APEL & Applied Processing Engineering Laboratory \\
\hline ARCM & AMEC radioactive crucible melt \\
\hline ASCM & AMEC simulant crucible melt \\
\hline ASTM & American Society for Testing and Materials \\
\hline APEL & Applied Processing Engineering Laboratory \\
\hline ASTM & American Society for Testing and Materials \\
\hline CFR & Code of Federal Regulations \\
\hline $\mathrm{CHG}$ & CH2M Hill Hanford Group \\
\hline DIW & deionized water \\
\hline DOE & U.S. Department of Energy \\
\hline EDS & energy dispersive spectroscopy \\
\hline EPA & U.S. Environmental Protection Agency \\
\hline ES & engineering-scale \\
\hline GDL & Glass Development Laboratory \\
\hline GDL-ECC & Glass Development Laboratory-Electrical Conductivity Calibration \\
\hline GDL-ELC & Glass Development Laboratory-Electrical Conductivity \\
\hline GDL-GBM & Glass Development Laboratory-Glass Batching and Melting \\
\hline GDL-VIS & Glass Development Laboratory-Viscosity \\
\hline GDL-VSC & Glass Development Laboratory-Viscosity Calibration \\
\hline HLW & high-level waste \\
\hline IA & image analysis \\
\hline $\mathrm{ICP}$ & inductively coupled plasma \\
\hline ICV & in-container vitrification \\
\hline ISE & ion selective electrode \\
\hline ISV & in-situ vitrification \\
\hline LAW & low-activity waste \\
\hline LOI & loss on ignition \\
\hline LOD & loss on drying \\
\hline LRM & LAW reference material \\
\hline LS & large scale \\
\hline MS & mass spectroscopy \\
\hline NIST & National Institute for Standards and Technology \\
\hline NQARD & Nuclear Quality Assurance Requirements Description \\
\hline $\mathrm{OM}$ & optical microscopy \\
\hline ORP & Office of River Protection \\
\hline PA & Performance Assessment (Hanford Site) \\
\hline PCT & product consistency test \\
\hline PTFE & Polytetrafluoro-ethlene \\
\hline PNNL & Pacific Northwest National Laboratory \\
\hline QA & quality assurance \\
\hline QC & quality control \\
\hline RCRA & Resource Conservation and Recovery Act \\
\hline redox & oxidation reduction \\
\hline $\mathrm{SC}$ & slow cooling \\
\hline
\end{tabular}


SEM scanning electron microscopy

SOW statement of work

SRTC Savannah River Technology Center

$\mathrm{S} / \mathrm{V}$

surface area-to-volume ratio

TCLP Toxicity Characteristic Leach Procedure

TIC total inorganic carbon

TOC total organic carbon

UTS Universal Treatment Standard

UV-VIS-NIR ultraviolet visible near infrared

VHT vapor hydration test

WSRC Westinghouse Savannah River Company

WTP Waste Treatment Plant

XRD X-ray diffraction 


\section{Contents}

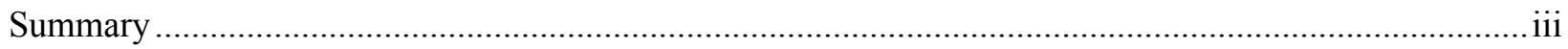

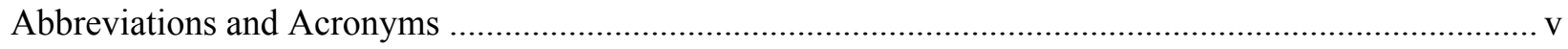

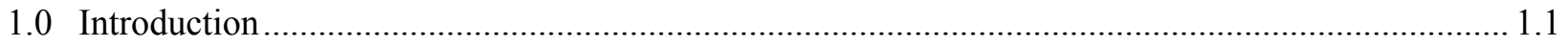

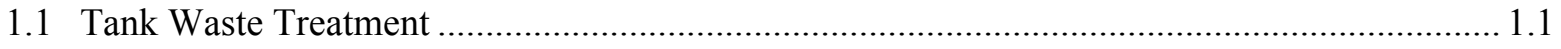

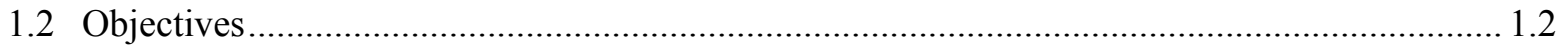

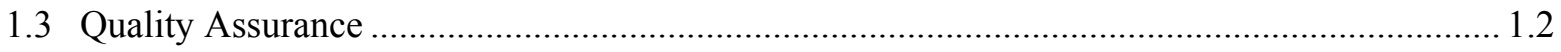

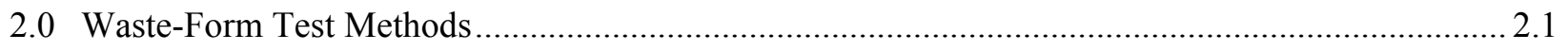

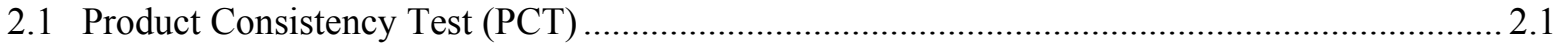

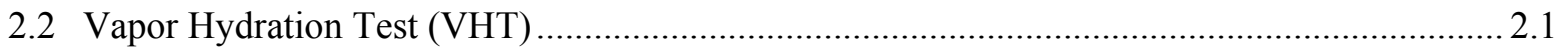

2.3 Toxicity Characteristic Leach Procedure (TCLP) …......................................................... 2.1

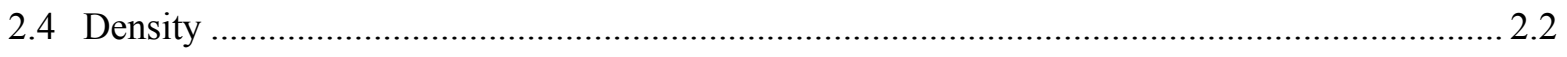

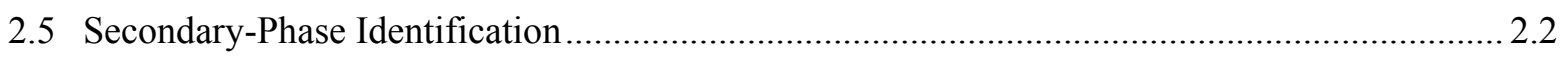

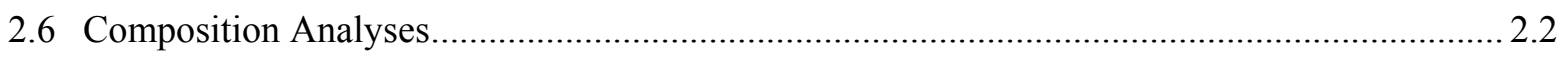

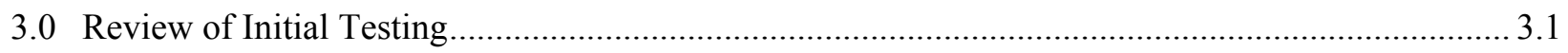

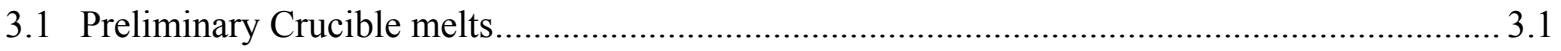

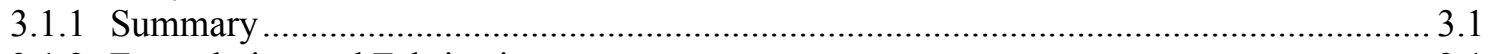

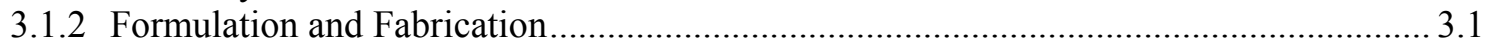

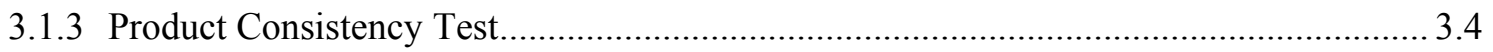

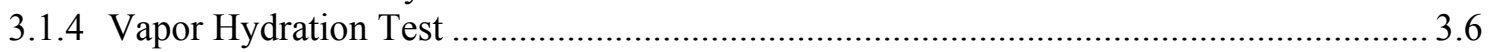

3.1.5 Toxicity Characteristic Leach Procedure .............................................................. 3.9

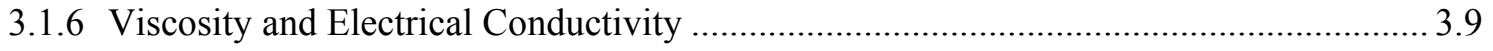

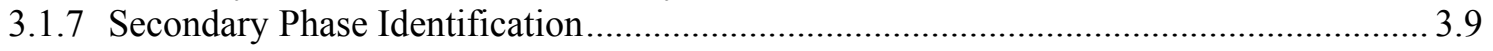

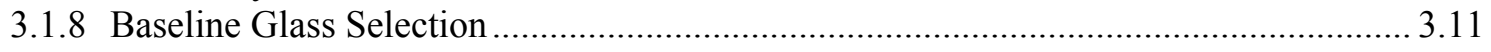

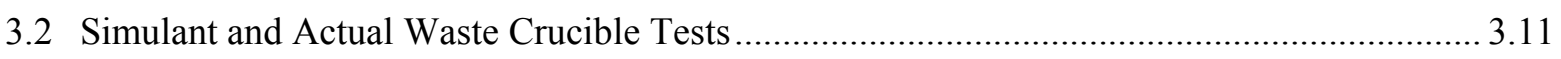

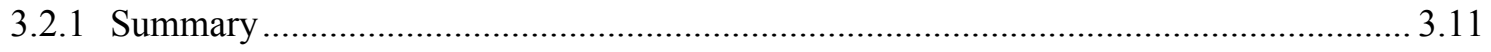

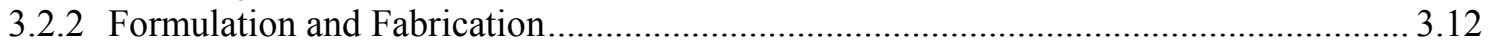

3.2.3 Glass Composition and Summary of Properties ..................................................... 3.13

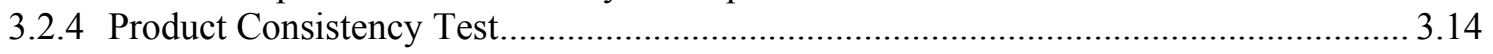

3.2.5 Vapor Hydration Test and Toxicity Characteristic Leach Procedure ............................ 3.17

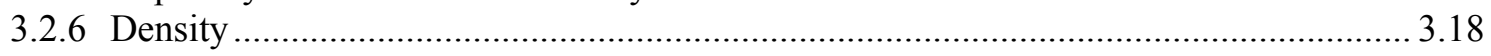

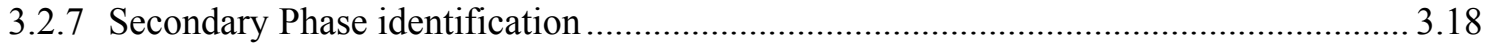

3.3 Engineering-Scale Simulant and Tc Tracer Tests ........................................................... 3.19 


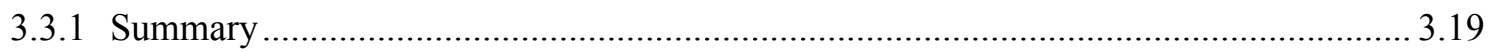

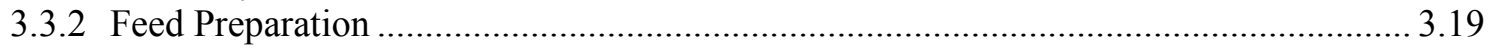

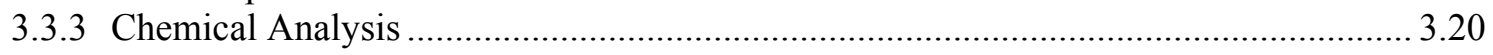

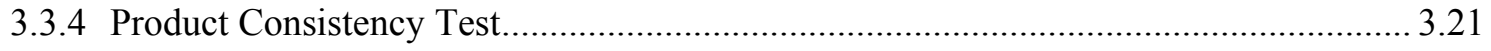

3.3.5 Vapor Hydration Test and Toxicity Characteristic Leaching Procedure ....................... 3.22

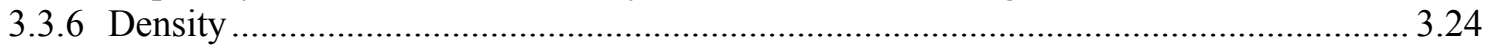

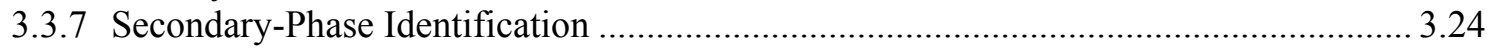

3.3.8 Sample Position and Identification for ES-2 Glass Composition Analyses .................. 3.25

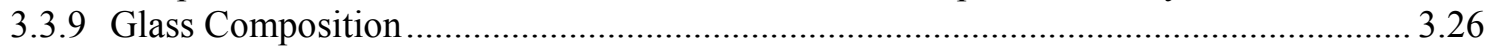

3.4 Initial Testing Summary and Conclusions .................................................................. 3.27

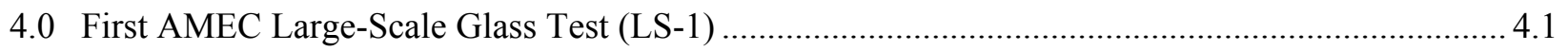

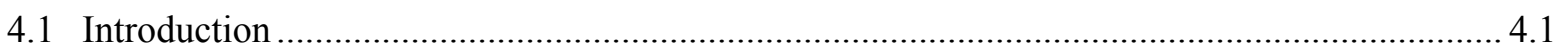

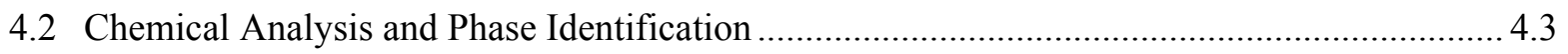

4.3 Vapor Hydration Test and Toxicity Characteristic Leach Procedure................................... 4.16

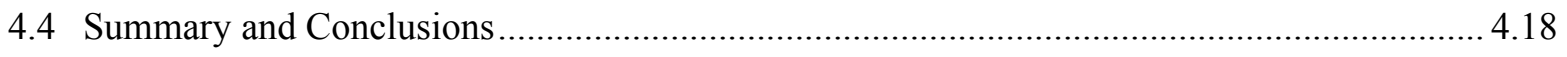

5.0 Second AMEC Large-Scale Glass Test (LS-2) ................................................................. 5.1

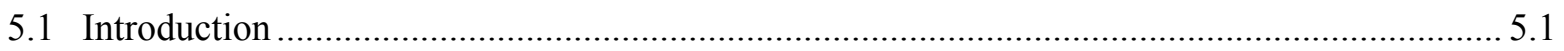

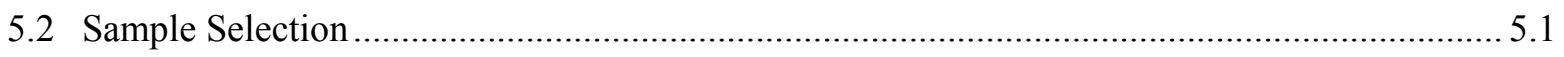

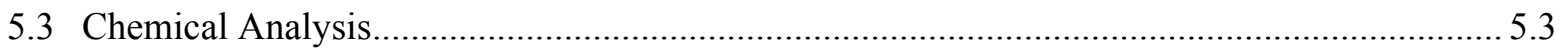

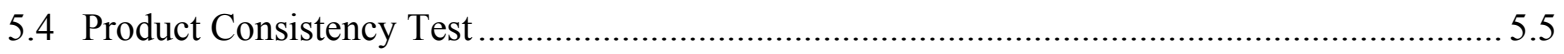

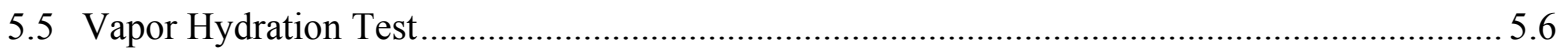

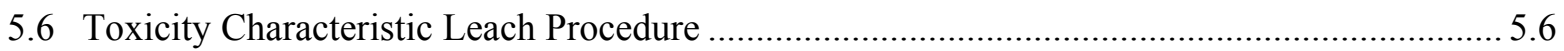

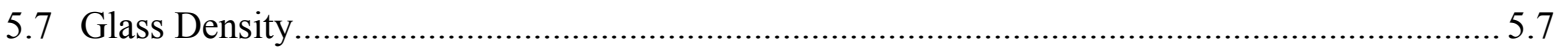

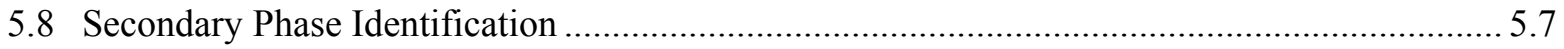

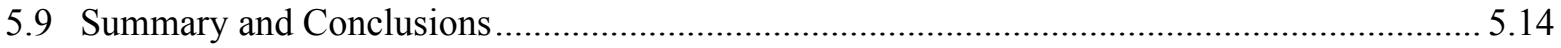

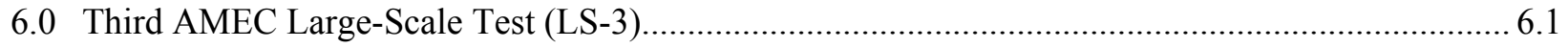

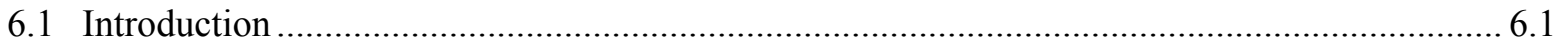

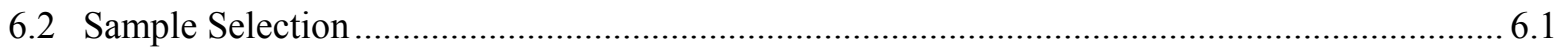

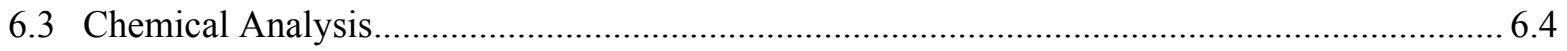

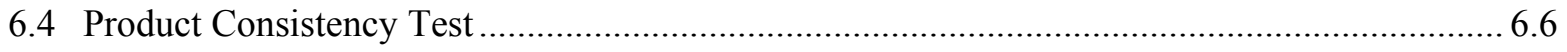




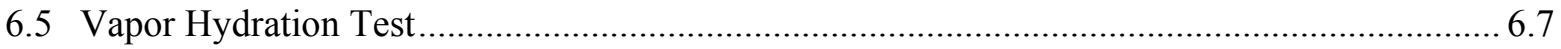

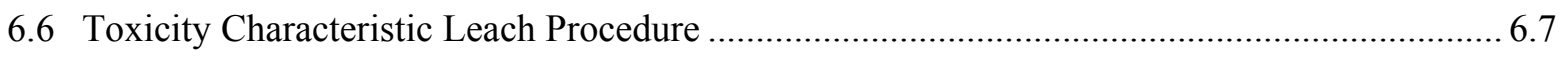

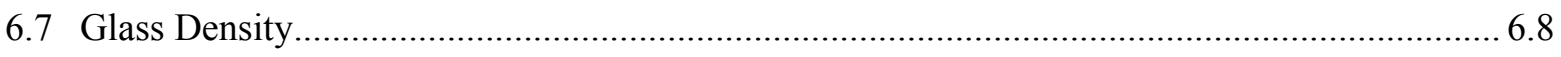

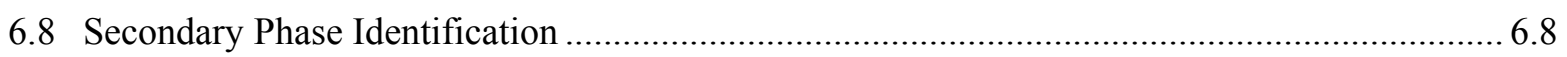

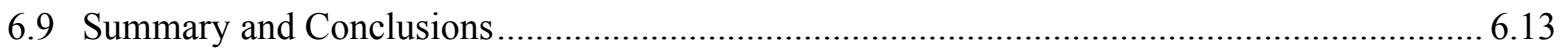

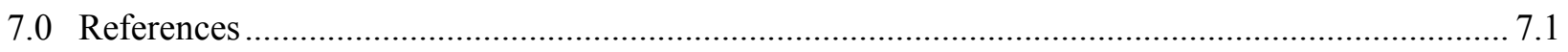

Appendix: PNNL Technical Procedures Used.............................................................................. 1 


\section{Figures}

Figure 1.1. Current ORP Schematic (from Allen et al. 2002) ............................................................ 1.1

Figure 3.1. PCT Releases (in $\mathrm{g} / \mathrm{m}^{2}$ ) versus Na Release (Quenched Samples) ........................................ 3.4

Figure 3.2. PCT Releases (in $\mathrm{g} / \mathrm{m}^{2}$ ) versus Na Release (SC Samples) ................................................

Figure 3.3. Effect of SC Treatment on Normalized Na Release ........................................................ 3.6

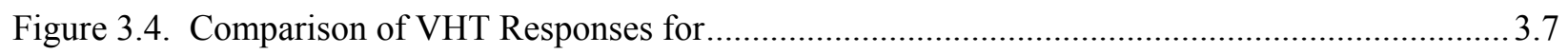

Figure 3.5. Comparison of VHT Corrosion Rates in Quenched and SC Samples ...................................3.7

Figure 3.6. VHT Effect of the Oxidation-Reduction State of Iron on the VHT Mass Loss for AMOG

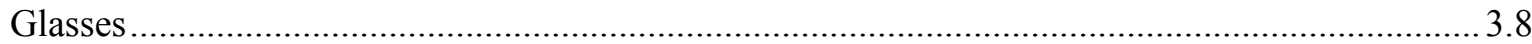

Figure 3.7. TCLP Normalized Releases for AMBG-13 and Typical WTP Glasses ............................. 3.10

Figure 3.8. SEM Micrograph Showing the Nepheline and Zirconia Crystals Formed at the Surface Layer

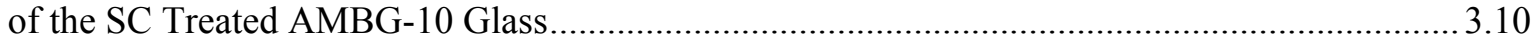

Figure 3.9. Optical Micrographs Showing Baddeleyite Crystals Formed and Settled at the Bottom of the

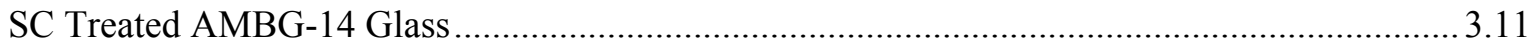

Figure 3.10. SEM Micrographs of Baddeleyite Crystals in Slow-Cooled AMBG-14 Glass ..................3.11

Figure 3.11. The Ratio of Measured to Target Concentrations for Selected Major Components in ASCM Glasses

Figure 3.12. Targeted Mass Fractions of Components in Simulated (ASCM-01) and Radioactive

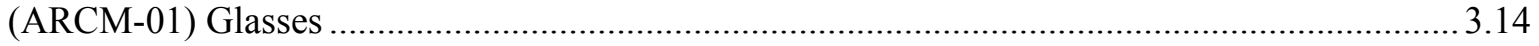

Figure 3.13. Analytical Mass Fractions of Components in Simulated (ASCM-01) and Radioactive

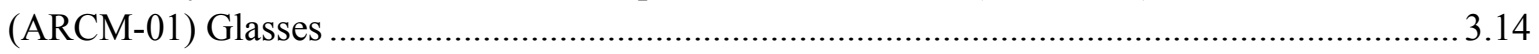

Figure 3.14. 7-Day PCT $r_{\mathrm{Na}}$ as a Function of $\mathrm{Na}_{2} \mathrm{O}$ Concentration in ASCM Glasses.......................... 3.16

Figure 3.15. Comparison of PCT Elemental Releases of Various Components from Quenched ASCM

Glasses with $\mathrm{Na}$ Release (the dashed line corresponds to $r_{i}=2 \mathrm{~g} / \mathrm{m}^{2}$ ) ......................................16

Figure 3.16. Comparison of PCT Elemental Releases of Various Components from SC Treated ASCM

Glasses with Na Release (the dashed line corresponds to $r_{i}=2 \mathrm{~g} / \mathrm{m}^{2}$ ) ..................................16

Figure 3.17. 7-Day PCT Normalized Releases from Simulated (ASCM-01) and Radioactive (ARCM-01) Quenched Glasses 
Figure 3.18. 7-Day PCT Normalized Releases from Simulated (ASCM-01) and Radioactive (ARCM-

01) SC Glasses. 3.17

Figure 3.19. VHT $r_{a}$ as a Function of $\mathrm{Na}_{2} \mathrm{O}$ Concentration in ASCM Glasses.....................................3.18

Figure 3.20. TCLP $r_{B}$ as a Function of $\mathrm{Na}_{2} \mathrm{O}$ Concentration in ASCM Glasses...................................... 3.18

Figure 3.21. Density as a Function of $\mathrm{Na}_{2} \mathrm{O}$ Concentration in ASCM Glasses ................................... 3.18

Figure 3.22. SEM Micrograph of Inclusions in SC-Treated ASCM-02 Glass ....................................... 3.19

Figure 3.23. SEM Image of an Inclusion in SC-Treated ASCM-02 Glass ..............................................19

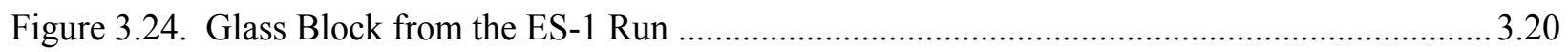

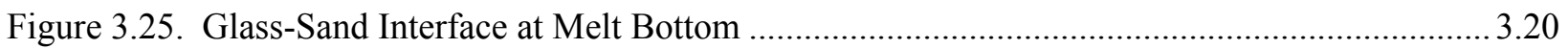

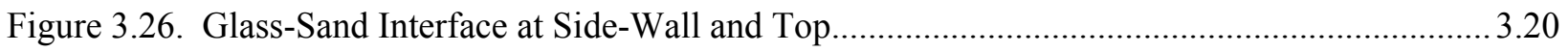

Figure 3.27. Ratio of Measured and Target Concentrations of $\mathrm{ReO}_{2}, \mathrm{SO}_{3}$, and Selected Major

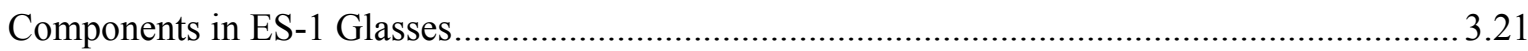

Figure 3.28. ES-1 Glass Block Showing the Areas from Where PCT, VHT, TCLP and Density Samples Were Obtained ${ }^{0}$

Figure 3.29. Illustration of How the ES-1-8 Samples for Glass Characterization Were Obtained from the Side-Wall Area of the ES-1 Glass Block ${ }^{0}$. 3.23

Figure 3.30. Optical Micrographs of VHT Coupons from Sand-Glass Interface Area, ES-1-8 ............ 3.23

Figure 3.31. Optical Micrographs of VHT Coupons from Sandy Area (ES-1-7S, left) and Sand-Glass Interface Area (ES-1-7M, right) after 14 days at $200^{\circ} \mathrm{C}$ (coupon size is $10 \mathrm{~mm}$ )

Figure 3.32. VHT Coupon from Glass near Electrode 3 (ES-1-10) with Metallic Droplets at the Contact with Electrode (iron was oxidized during the 14-days exposure to steam at $200^{\circ} \mathrm{C}$ ) .................... 3.25

Figure 3.33. A Typical Section of Glass with Metallic Droplets (SEM micrograph).......................... 3.25

Figure 3.34. Metallic Droplets Released from ES-1 Glass Block During Sampling ............................ 3.25

Figure 3.35. $\mathrm{SiO}_{2}$ Concentration as a Function of Distance from the Surface of the ES-1 Block .......... 3.25

Figure 3.36. Ratio of Measured and Target Concentrations of ES-2 Glasses Components....................3.26

Figure 3.37. SEM Micrographs of White Condensate on the Inside of Large Glass Bubbles from ES-1

Melter Test (similar to the condensate from ES-2 that was dissolved in DIW for analyses) .......3.27

Figure 4.1. Interfacial Area between Silica-Sand Lining and LS-1 Glass Block. 
Figure 4.2. A Fraction of the LS-1 Block with the Top Surface

Figure 4.3. LS-1-B Sample Taken From the Top of LS-1 Block (see Figure 4.2) .....

Figure 4.4. XRD and Optical Micrographs of LS-1-B-R Sample (purple area, see Figure 4.3) (inset and bottom right reflected light, bottom left transmitted light showing voids in the material)......

Figure 4.5. Optical Micrographs of LS-1-B-F sample (see Figure 4.3) Showing Amorphous phase with the Formation of Unidentified Crystals....

Figure 4.6. Optical Micrographs of LS-1-B-G Sample (see Figure 4.3) Showing the Sand-Glass Interface and Crystal Formation in Glass .....

Figure 4.7. Large $(\sim 30-\mathrm{cm})$ Pieces of Material from the Top Portion of the LS-1 Block 4.8

Figure 4.8. XRD Analysis of LS-1-R, the Red Sample Shown in Figure 4.7. 4.8

Figure 4.9. XRD Analysis of LS-1-T, the Tan Sample Shown in Figure 4.7.

Figure 4.10. XRD Analysis of LS-1-F Sample from the Foamy Layer on the Top of the LS-1 Block .... 4.9

Figure 4.11. A Sample of LS-1 Glass from the Block Bottom with Metallic Droplets. Massive Droplets (over $2 \mathrm{~cm}$ in diameter) are Visible on the Left Photograph, the Rough Surface of which is Displayed on the Stereo-Microscope Image on the Right

Figure 4.12. XRD Pattern of Precipitate from the LS-1 Yellow Layer Leachate (shown in the inset) .. 4.10

Figure 4.13. Blue Layer Sample Analyzed in Table 4.4

Figure 4.14. Cross Section through Glass-Silica Sand Interface (the thickness from let to right is $58 \mathrm{~mm}$ )

Figure 4.15. Glass with White Crystals of Baddeleyite Adjacent to the Interface with Silica 4.13

Figure 4.16. Silica Particles Permeated with Glass and Tridymite Crystals in the Gray Layer. .4 .13

Figure 4.17. Silica Particles with Glass and Pores in the Blue Layer..... 4.14

Figure 4.18. Silica Particles with Glass and Pores in the Yellow Layer.

Figure 4.19. Silica Particles in the White Layer.....

Figure 4.20. SEM Micrograph of the Amorphous Phase in the LS-1 Yellow Layer with Undissolved

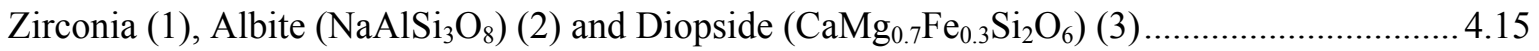

Figure 4.21. SEM Micrograph of LS-1 Blue Layer with Crystals of Tridymite $\left(\mathrm{SiO}_{2}\right)$ 4.16

Figure 4.22. SEM Micrograph of LS-1 Blue Layer with Crystals of Cristobalite $\left(\mathrm{SiO}_{2}\right)$ 4.16 
Figure 4.23. Optical Micrograph of LST-1 VHT Coupon Prior to Test Initiation

Figure 4.24. Optical Micrographs of LS-1 VHT Coupon Cross Sections after Testing for (a) 7-days and

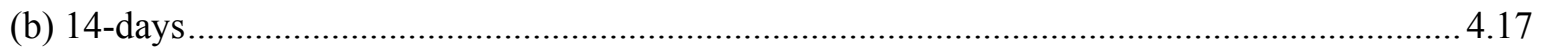

Figure 5.1. Photographs Depicting Sample Locations From LS-2 the Test ........................................ 5.3

Figure 5.2. Ratio of Measured and Target (Adjusted) Concentrations Selected Major Components in LS2 Glasses.....

Figure 5.3. SEM Micrographs of Crystals in Sample LS-2-03-200-014: Individual Crystals of Zircon (a to c) and Zircon Crystals ( 1 in d) Nucleated on and Dissolving Zirconia (2 in d) .........................5.8

Figure 5.4. SEM Micrographs of Crystals in Sample LS-2-05(1)-200-014: Zircon Crystals (1) with Residual Zirconia (2)

Figure 5.5. SEM Micrographs of Crystals in Sample LS-2-05(1)-200-014: Tridymite Crystals Originated from Residual Sand Grains; a Tiny Crystal of Zircon (1) is Seen in (a) ......................................5.10

Figure 5.6. EDS Dot Map of a Cluster of Zircon Crystals Growing from Dissolving Zirconia in Sample LS-2-05(01)-200-014 5.11

Figure 5.7. EDS Dot Map an Iron-Rich Inclusion, a Diopside Crystal, and Zircon Crystals in Sample LS2-05(01)-200-014 5.12

Figure 5.8. XRD Pattern of Samples LS-2-03 and LS-2-05 5.13

Figure 6.1. Photograph of LS-3 Glass Block 6.2

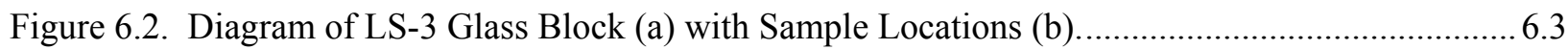

Figure 6.3. Photographs of LS-3 Block with Sample Locations Marked. 6.4

Figure 6.4. Ratio of Measured and Target (Adjusted) Concentrations Selected Major Components in LS3 Glasses

Figure 6.5. A Portion of LS-3 Glass Block Close to the Top Area Used for XRD Analysis

Figure 6.6. A Plot of Crystalline Phase Concentrations in LS-3 Glass Block Samples against Sample Location. 6.10

Figure 6.7. A Portion of LS-3 Glass Block Showing the Location of Sand Layer Samples for Composition Analyses

Figure 6.8. Regions of LS-3 Glass Block Used for SEM/EDS Analyses

Figure 6.9. Dissolving $\mathrm{ZrO}_{2}$ (1) and Crystals of Zircon (2) Observed in Region 1 of LS-3 Glass Block Sample in Figure 6.8 . 
Figure 6.10. Sand Particles and Glassy Area with Crystals in Region 2 of LS-3 Glass Block Sample in

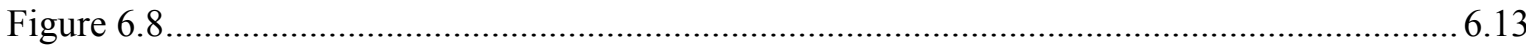

Figure 6.11. Crystals of $\mathrm{SiO}_{2}$ Polymorphs (Presumably Tridymite and Cristobalite) in Region 3 of LS-3

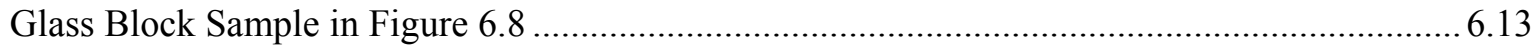




\section{Tables}

Table 2.1. Acceptable Concentrations of Some Restricted Metals in TCLP Solution............................2.1

Table 3.1. Local Soil and Waste-Simulant Compositions in Mass Fractions .......................................... 3.2

Table 3.2. Compositions of AMBG Test Glasses in Mass Fractions..................................................... 3.2

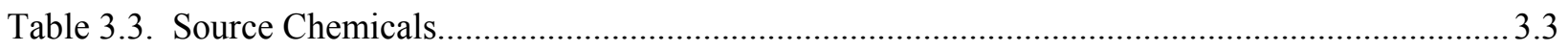

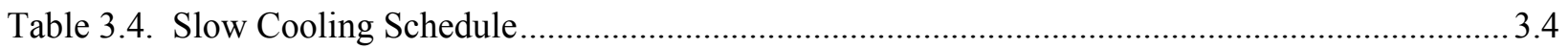

Table 3.5. PCT Normalized Releases in $\mathrm{g} / \mathrm{m}^{2}$ from AMBG Glasses ..................................................... 3.5

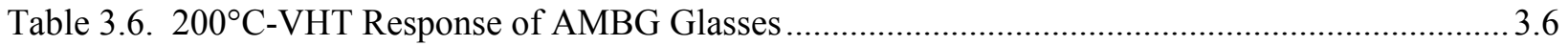

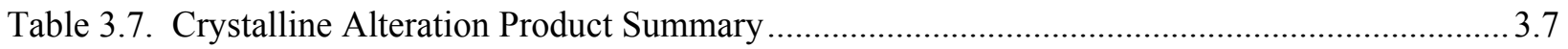

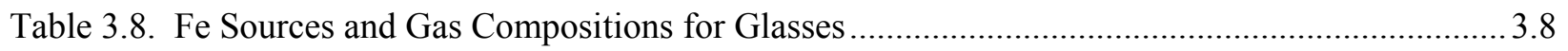

Table 3.9. Measured Redox Ratio for AMOG Samples and $200^{\circ} \mathrm{C}$ VHT Mass Losses in $\mathrm{g} / \mathrm{m}^{2}$............. 3.8

Table 3.10. TCLP Responses (in mg/L) of Selected AMBG Glasses................................................. 3.9

Table 3.11. Viscosity Results for AMBG-13 and 16 Glasses ............................................................ 3.10

Table 3.12. Electrical Conductivity Results for AMBG-13 and 16 Glasses.........................................10

Table 3.13. Crystalline Phases, in Mass\%, Determined by XRD in SC Samples within $\sim 1.5-\mathrm{mm}$ at Pt-

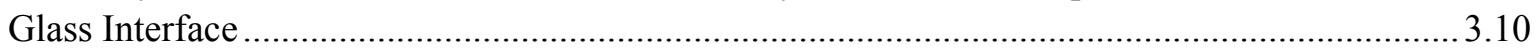

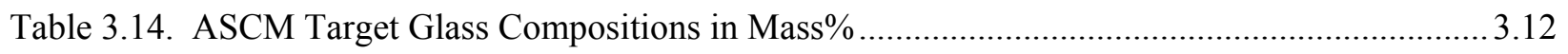

Table 3.15. Compositions of ASCM and ARCM Glasses (in mass fractions) ........................................12

Table 3.16. Analyzed Compositions of ASCM and ARCM Glasses (in mass fractions) ${ }^{(a)}$....................13

Table 3.17. Summary of Properties for Simulant and Actual Waste Crucible Test Glasses .................. 3.15

Table 3.18. Concentration of Tc in PCT Solutions from ARCM-01 Glasses and Normalized Tc Releases

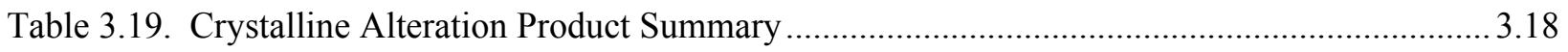

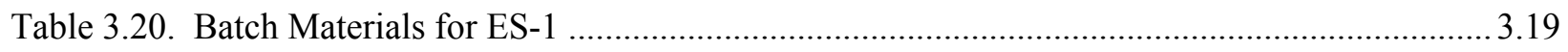

Table 3.21. Amount of Flux Materials Used in Each ES Test ................................................................2.20 
Table 3.22. Analyzed Compositions (in mass fractions) and Redox of ES-1 Glasses.

Table 3.23. PCT Normalized release $\left(\mathrm{g} / \mathrm{m}^{2}\right)$ for ES-1 Glasses 3.22

Table 3.24. 14-day VHT Responses for ES-1 Glasses...... 3.23

Table 3.25. TCLP Responses for ES-1 Glasses 3.24

Table 3.26. Density of ES-1 Glass Samples (g/mL) 3.24

Table 3.27. Analyzed Compositions (in mass fraction) of ES-2 Glasses 3.26

Table 4.1. Composition of Simulant, Soil, and Sand Used in LS Tests.

Table 4.2. Amount of Batch Feed, Flux, and Starter Path Materials Used in LS-1 Test.....

Table 4.3. Target and Measured Compositions of LS-1 Glass in Mass Fractions 4.2

Table 4.4. Measured Composition of the Blue Sample and Estimated Composition of the Salt in the Sample

Table 4.5. Estimated Mass Fractions of Batch Materials in the Blue Layer Sample 4.11

Table 4.6. Used and Calculated Compositions of Waste in the Blue Layer Sample

Table 4.7. Measured VHT Responses for LS-1 Specimen

Table 5.1. Amount of Batch Feed, Flux, and Starter Path Materials Used in the LS-2 Test 5.2

Table 5.2. Target Compositions of LS-2 Glass. 5.2

Table 5.3. Measured Compositions of LS-2 Glass Samples (in mass fractions) 5.4

Table 5.4. Estimated Mass Fraction of Sand Dissolved in the LS-2 Glass Samples (S) Calculated Based on Analyzed Glass Compositions

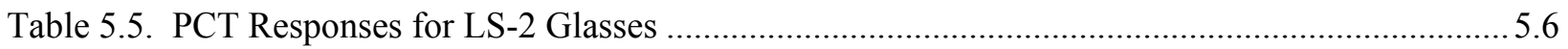

Table 5.6. Measured VHT Responses for LS-2 Specimens ................................................................ 5.6

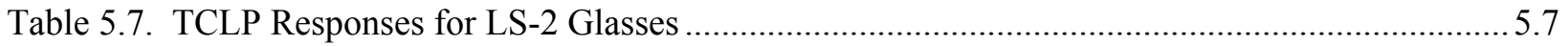

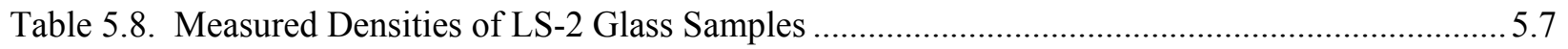

Table 5.9. Crystallinity Content in Samples LS-2-03 and LS-2-05 by XRD.....................................5.13

Table 6.1. Amount of Batch Feed, Flux, and Starter Path Materials Used in LS-3 Test ....................... 6.1

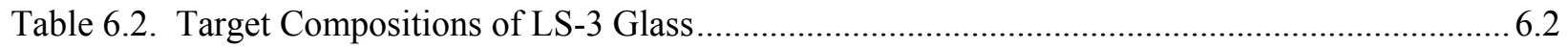


Table 6.3. Measured Compositions of LS-3 Glass Samples (in mass fractions)

Table 6.4. Estimated Mass Fraction of Sand Dissolved in the LS-3 Glass Samples (S) Calculated Based

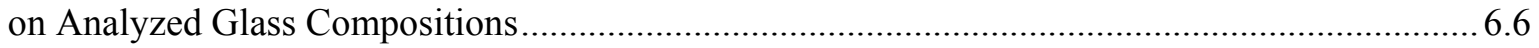

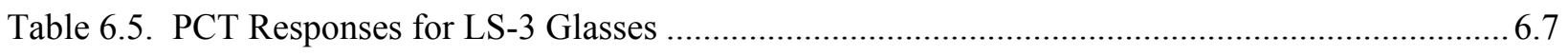

Table 6.6. Measured VHT Responses for LS-3 Specimens ............................................................ 6.7

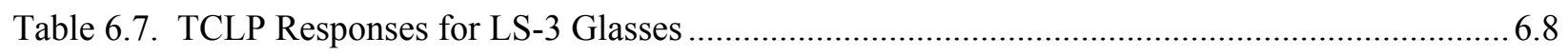

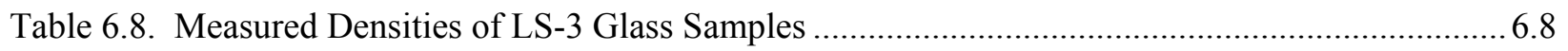

Table 6.9. Crystalline Phase Concentrations in LS-3 Glass Block Samples.......................................... 6.9

Table 6.10. Measured Compositions of Sand Layers in LS-3 Glass Block ............................................ 6.11 


\subsection{Introduction}

\subsection{Tank Waste Treatment}

Roughly 51 million gallons (232-thousand $\mathrm{m}^{3}$ ) of radioactive waste are stored in 177 underground tanks at the Hanford site in central Washington. This waste was generated from over four decades of heavy metal separations and nuclear materials processing conducted by the U.S. Department of Energy (DOE) and its predecessors. The DOE Office of River Protection (ORP) plans to retrieve the tank waste, separate it into low-activity waste (LAW) and high-level waste (HLW) fractions and separately treat the waste for disposal. Figure 1.1 shows a schematic of the current ORP flowsheet This process includes the retrieval and delivery of tank waste to the Waste Treatment Plant (WTP), at which the HLW and LAW fractions will be separated and separately vitrified (BNI 2003). However, the current planned capacity of the WTP is only a fraction of the LAW that can be treated by the scheduled completion date of 2028. Therefore, ORP, through its tank farm operator CH2M Hill Hanford Group (CHG), is considering supplemental treatment technologies for LAW. There are two possible insertion points for the supplemental treatment technologies in the current strategy: 1) divert the LAW radioactive waste tanks directly to the supplemental treatment, which may or may not include radionuclide separations steps, and 2) divert the LAW fraction of tank waste from the WTP to the supplemental treatment technology. By either option, the waste will be treated for disposal in the Hanford Site Near-Surface Burial Facility for retrievable disposal.

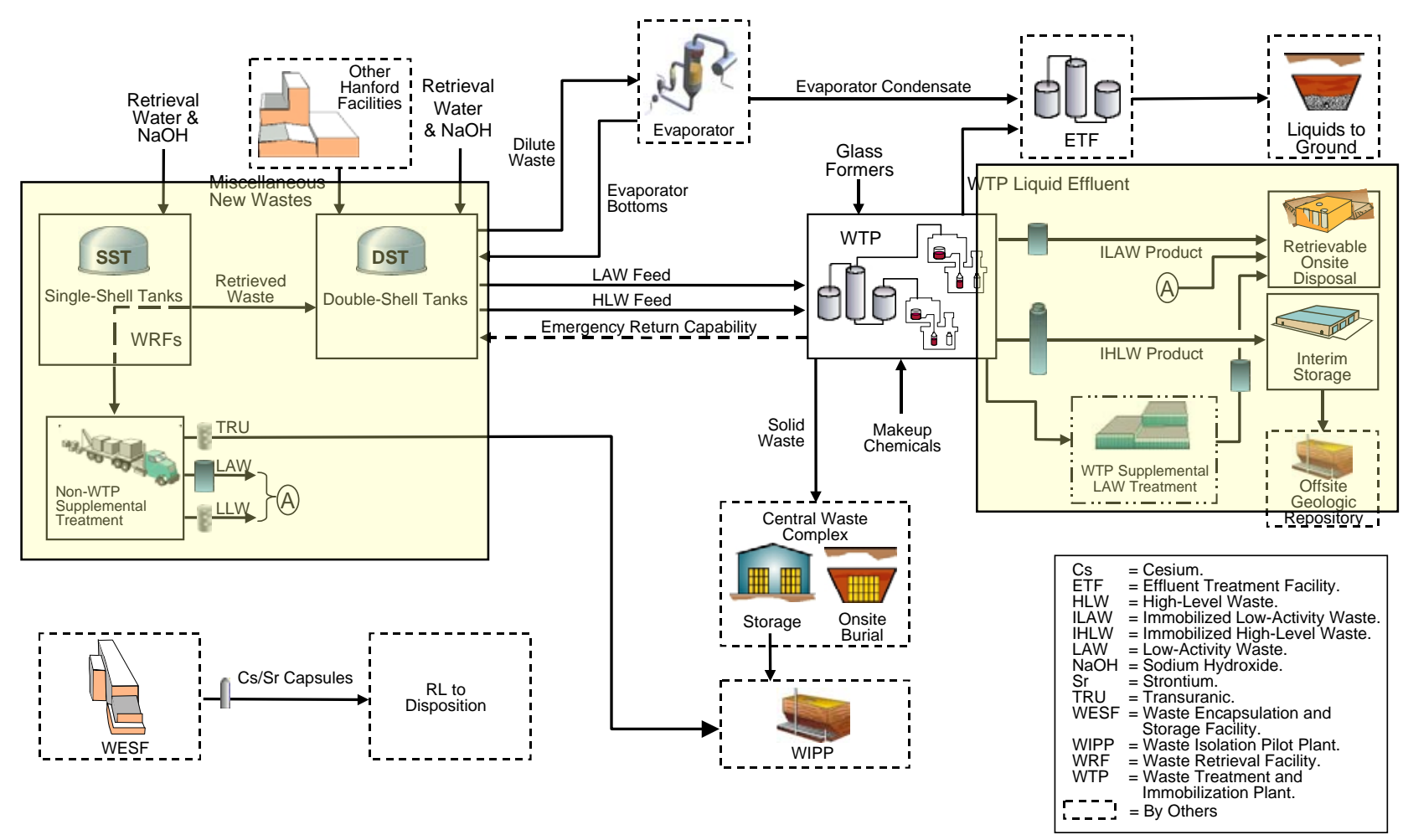

Figure 1.1. Current ORP Schematic (from Allen et al. 2002) 
One of the supplemental treatment technologies being considered by CHG and ORP is in-container vitrification (ICV). The ICV is a technology developed by AMEC, GeoMelt Division, based on in situ vitrification (ISV, Buelt et al. 1987; Geosafe 1998; Spalding et al. 1992). In this technology, the waste is mixed with soil and possibly additional additives, dried, and loaded into an insulated metal box. The dried mixture is melted with current supplied by two graphite electrodes imbedded in the batch. The process is fundamentally similar to the WTP LAW vitrification process with a few key differences. The melter used in the ICV is also the disposal container, reducing the concern for corrosion-related processes lowering the melter life. Therefore, the ICV can operate, and is typically operated, at higher temperatures than the WTP process, thus avoiding molten salt accumulation that limits the loading of higher sulfur LAWs in WTP glass. ICV waste-form composition can be lower in fluxes that must be used in the WTP to maintain adequate transport properties at lower temperatures. The ICV waste-form is typically an alumino-silicate glass but can include boron or other components if desired to maintain adequate properties (such as chemical durability). Since the ICV "melter" is used only once and the glass is not poured into a different container, the process is more tolerant of crystalline phase formation in the melt. Therefore, secondary phases are not detrimental to the waste-form as long as they do not cause the properties of the overall waste form to fail any performance requirements. Since the ICV product is primarily composed of soil and waste, the materials cost is significantly lower than that of the WTP LAW vitrification, in which high-cost chemicals are used to produce a glass.

Generally, the chemical durability of the LAW glass produced with the ICV process is expected to be better than that of the WTP process for comparable waste loadings. The improved durability is possible as a result of the capability to operate at higher temperatures than the WTP process. With higher temperatures, lower concentrations of non-waste flux components are required to maintain adequate melt viscosity and electrical conductivity. The lower flux concentrations generally lead to higher chemical durability.

\subsection{Objectives}

AMEC contracted with Pacific Northwest National Laboratory (PNNL) for technical support in wasteform development and product testing for a process demonstration with Hanford LAW. In the initial phase of the testing, the baseline glass was formulated. Versions of this glass with simulated and actual waste were prepared and tested. Engineering-scale simulant testing with a Tc surrogate and Tc tracer were accomplished. The objective of this study is testing glasses produced in large-scale tests performed by AMEC and their contractors as described in the project Demonstration and Test Plan (AMEC 2002).

\subsection{Quality Assurance}

The work described in this report was performed under the PNNL Nuclear Quality Assurance Requirements Description (NQARD) procedures in accordance with the AMEC Bulk Vitrification Project Quality Assurance Plan. These quality assurance procedures and program plan are compliant with the NQA-1 quality assurance (QA) program requirements passed down in the statement of work. ${ }^{(a)}$

(a) Statement of Work, Supplemental Technology - Bulk Vitrification, Requisition \#93505, "Phase I - Bulk Vitrification", Rev.0, dated October 2, 2002, CH2M Hill Hanford Group. 


\subsection{Waste-Form Test Methods}

The experimental methods used to characterize the waste-form samples from each task are summarized in this section. The Appendix lists the PNNL procedures used.

\subsection{Product Consistency Test (PCT)}

The product consistency test (PCT) was performed as defined in American Society for Testing and Materials (ASTM) C 1285 (ASTM 1998). Glass was ground, washed, and mixed with deionized water (DIW) at a surface area-to-volume ratio (S/V) of approximately $2000 \mathrm{~m}^{-1}$. The 7-day test was performed in sealed stainless steel vessels (Type 304L) at $90 \pm 2{ }^{\circ} \mathrm{C}$. Solutions were then filtered, acidified, and analyzed for $\mathrm{Si}, \mathrm{Na}$, and selected other components (e.g., B) with inductively coupled plasma-atomic emission spectroscopy (ICP-AES).

\subsection{Vapor Hydration Test (VHT)}

In the vapor hydration test (VHT), monolithic samples were exposed to water vapor at $200^{\circ} \mathrm{C}$ in sealed stainless steel vessels (Type $304 \mathrm{~L}$ ). A diamond-impregnated saw is used to produce samples, $10 \times 10 \times 1.5$ $\mathrm{mm}(0.4 \times 0.4 \times 0.06 \mathrm{in}$.), from glass bars. The cut samples are polished to 600 -grit surface finishes with silicon carbide paper. In the vessels, samples were suspended on Pt[?] wire above $0.20 \mathrm{~g}$ of DIW water and held at $200^{\circ} \mathrm{C}$ for a predetermined amount of time (typically 14 days). Specimens were then sectioned for optical microscopy/image analysis (OM/IA) evaluation, and the corrosion products were tested for phase identification with X-ray diffraction (XRD) and scanning electron microscopy/energy dispersive spectroscopy (SEM/EDS). The average remaining glass thickness, $m$, was measured, and the average rate of corrosion was calculated as $r_{a}=m / t$, where $t$ is the corrosion time. An estimated measurement uncertainty of $m$ is $\pm 10 \mathrm{~g} / \mathrm{m}^{2}$, e.g., $m$ value of $\pm 10 \mathrm{~g} / \mathrm{m}^{2}$ means zero response.

\subsection{Toxicity Characteristic Leach Procedure (TCLP)}

The toxicity characteristic leach procedure (TCLP) testing was performed at Severn Trent Laboratories, Inc. (STL St. Louis, 13725 Rider Trail North, Earth City, MO 63045). The extraction and analyses was performed according to SW 846 method 1311 (EPA 1992) and quality assurance/quality control requirements. Glass pieces, $\leq 9.5 \mathrm{~mm}$ ( $0.4 \mathrm{in}$.) in size and $\geq 100 \mathrm{~g}$ in mass, were placed in dilute acetic acid ( $\mathrm{pH}$ value of $4.98 \pm 0.05)$ and agitated at $30 \pm 2 \mathrm{rpm}$ for $18 \pm 2$ hours at room temperature. The concentrations of hazardous metals in solution were then measured. Table 2.1 lists values specified by the Resource Conservation and Recovery Act (RCRA) and by the Universal Treatment Standard (UTS) put forth by the U.S. Environmental Protection Agency (EPA) (40 CFR 268.48) for certain waste categories defined in 40 CFR 268.40 in May 1998.
Table 2.1. Acceptable Concentrations of Some Restricted Metals in TCLP Solution

\begin{tabular}{||c|c|c||}
\hline Metal & $\begin{array}{c}\text { Characteristic } \\
\text { Limit } \\
\text { ppm }\end{array}$ & $\begin{array}{l}\text { UTS Limit } \\
\text { ppm }\end{array}$ \\
\hline $\mathrm{Ag}$ & 5 & 0.14 \\
\hline $\mathrm{As}$ & 5 & 5 \\
\hline $\mathrm{Ba}$ & 100 & 21 \\
\hline $\mathrm{Cd}$ & 1 & 0.11 \\
\hline $\mathrm{Cr}$ & 5 & 0.6 \\
\hline $\mathrm{Pb}$ & 5 & 0.75 \\
\hline $\mathrm{Se}$ & 1 & 5.7 \\
\hline $\mathrm{Zn}$ & --- & 4.3 \\
\hline $\mathrm{Ni}$ & --- & 11 \\
\hline $\mathrm{Sb}$ & --- & 1.15 \\
\hline $\mathrm{Be}$ & --- & 1.22 \\
\hline $\mathrm{Tl}$ & --- & 0.2 \\
\hline $\mathrm{V}$ & --- & 1.6 \\
\hline
\end{tabular}




\subsection{Density}

Glass density was measured with an Accupyc 1330 Gas Pycnometer according to the procedure APELPIP-4. Annealed glasses were cut to obtain solid specimens with a nominal volume sufficient to fill at least $10 \%$ of the sample chamber in the gas pycnometer. Samples were washed with DIW, placed into a glass beaker filled with ethanol, ultrasonically cleaned, and dried at $110^{\circ} \mathrm{C}$. The sample dry mass was determined with a calibrated four-decimal-place balance. The pycnometer was calibrated before and after the experiment with a tungsten carbide ball, which is a NIST-traceable standard. The average glass density was calculated after five runs for each glass.

\subsection{Secondary-Phase Identification}

The amount of crystallinity in glass was determined by the quantitative XRD. Though the actual detection limit depends on the type of the crystalline material, XRD can generally detect and quantify as little as $0.25 \mathrm{wt} \%$ of a crystalline phase in the glass. The PNNL procedure "Quantitative and SemiQuantitative Analysis using X-Ray Diffraction" (GDL-XRD) was followed. The internal standard for quantitative XRD is 5 mass $\% \mathrm{CaF}_{2}$. The sample mass of 1 to $2 \mathrm{~g}( \pm 0.0001 \mathrm{~g})$ was milled for $2 \mathrm{~min}$. in the tungsten-carbide milling chamber. The powder was mounted in an XRD sample holder. Scanning proceeded with $0.04^{\circ} 2-\theta$ step size, 6-s dwell time, and from 5 to $70^{\circ} 2-\theta$ scan range.

The secondary phase identification and quantification was aided by OM/IA and SEM/EDS. Glass samples were sliced and polished. Glass and crystal compositions were determined by EDS. Standard glasses were analyzed with each sample to provide reference composition for bias adjustments.

\subsection{Composition Analyses}

The chemical compositions of non-radioactive glasses were measured at Westinghouse Savannah River Company (WSRC, Aiken, SC 29808). Chemical and radiochemical compositions of radioactive glasses were measured by PNNL using similar procedures. Samples were fused in $\mathrm{KOH}$ and $\mathrm{Na}_{2} \mathrm{O}_{2}$, then dissolved in dilute nitric acid and analyzed with ICP-AES for major components and with ICP-mass spectroscopy (MS) for minor components (e.g., Tc and Re). Ion chromatography was used for $\mathrm{F}, \mathrm{SO}_{4}$, and $\mathrm{PO}_{4}$. The concentrations of $\mathrm{U}$ and $\mathrm{Cs}$ were determined with U-KPA and gamma energy analyses, respectively. In addition to quality control (QC) standards, samples of LRM-1 glass (Wolf et al. 1998) were included as blind-standard glasses for the analyses. The $\mathrm{Fe}^{2+} / \Sigma \mathrm{Fe}$ ratio was measured with wet colorimetry and ultraviolet visible near infrared (UV-VIS-NIR) spectrophotometry. 


\subsection{Review of Initial Testing}

The initial studies to formulate and test simulated and actual Hanford LAW glasses for the ICV process was performed in five phases:

1. Preliminary Crucible Melts

2. Simulant Crucible Melts

3. Radioactive Crucible Melt

4. Engineering Scale Simulant Melt

5. Engineering Scale Technetium Tracer Melt

This section reviews these studies and summarizes the main results.

\subsection{Preliminary Crucible melts}

\subsubsection{Summary}

A preliminary study was performed to identify a baseline glass that met the processing, product quality, and economic constraints of the ICV process applied to Hanford LAW. Sixteen glasses were formulated, fabricated, and tested. The key parameters varied were waste loading and additive composition (soil, $\mathrm{B}_{2} \mathrm{O}_{3}$, and $\mathrm{ZrO}_{2}$ ). All glasses were characterized for VHT and PCT responses and crystallinity (both quenched and slow-cooled samples), while selected glasses were tested for TCLP responses, viscosity, and electrical conductivity. The VHT response was found to be the most restrictive property on waste loading and glass composition. The AMBG-13 glass was adopted as suitable for scale-up and radioactive demonstrations of the ICV process because it has outstanding PCT, VHT, and TCLP responses, does not contain any crystals after slow cooling (SC) heat treatment, and has 20 mass $\% \mathrm{Na}_{2} \mathrm{O}$. The temperature at a viscosity of $10 \mathrm{~Pa} \cdot \mathrm{s}$ for this glass is $1238^{\circ} \mathrm{C}$, and electrical conductivity at this temperature is $39.7 \mathrm{~S} / \mathrm{m}$.

Since the glass is melted using graphite electrodes, a special study was conducted to determine the effect of the oxygen fugacity on the VHT response of the glass. Three melts were performed with varying redox ratios of ASCM-04 glass (which has an acceptable but borderline VHT response). The VHT response improved as the glass became more reduced.

\subsubsection{Formulation and Fabrication}

In the ICV process, the major additive to the waste is soil. Additions of $\mathrm{ZrO}_{2}$ and $\mathrm{B}_{2} \mathrm{O}_{3}$ were necessary for glasses to pass the VHT specifications because the soil contained high enough levels of $\mathrm{Al}_{2} \mathrm{O}_{3}$ to impair the VHT response. The 16 preliminary glass compositions were formulated in four groups to investigate the impacts of waste loading, soil composition variation (only $\mathrm{Al}_{2} \mathrm{O}_{3}$ and $\mathrm{Fe}_{2} \mathrm{O}_{3}$ content), and additive composition and concentration on key glass properties. The LAW waste simulant formulated by Rassat et al. (2003) was used as the single representative LAW composition selected for testing (Table 3.1). Glass formulations were focused on achieving target $\mathrm{Na}_{2} \mathrm{O}$ loadings in the vitrified product ranging from 17 to 26 mass\% with soil (of the composition of "AMEC Site" in Table 3.1) as the major additive. Glasses were formulated in four general sets (Table 3.2): 
1. Glasses AMBG-01 through 04 were soil-LAW mixtures that ranged from 17 to 26 mass $\% \mathrm{Na}_{2} \mathrm{O}$.

2. Glasses AMBG-05 through 08 contained 20 and 23 mass $\% \mathrm{Na}_{2} \mathrm{O}$ and 5 mass $\% \mathrm{ZrO}_{2}$ or 2.5 mass $\% \mathrm{ZrO}_{2}+2.5$ mass $\% \mathrm{~B}_{2} \mathrm{O}_{3}$ in addition to soil.

3. Glasses AMBG-09 through 12 were formulated with varied concentrations of $\mathrm{Al}_{2} \mathrm{O}_{3}$ and $\mathrm{Fe}_{2} \mathrm{O}_{3}$ keeping the same proportions of soil, $\mathrm{LAW}$, and $\mathrm{ZrO}_{2}$ as in AMBG-07.

4. Glasses AMBG-13 through 16 contained 5 mass $\% \mathrm{~B}_{2} \mathrm{O}_{3}$ and varied $\mathrm{ZrO}_{2}$ concentration. Glass AMBG-15 contained $\mathrm{P}_{2} \mathrm{O}_{5}, \mathrm{La}_{2} \mathrm{O}_{3}$, and $\mathrm{TiO}_{2}$ as additional additives, and AMBG- 16 contained 3 mass $\% \mathrm{SiO}_{2}$ as an additive.

The AMBG glasses were fabricated and tested in accordance with the procedure GDL-GBM. ${ }^{\text {(a) }}$ Chemicals used are listed in Table 3.3. Note that $\mathrm{FeO}$ was used as the source of iron in these preliminary melts because the ICV process is expected to operate in a relatively reducing mode.

Table 3.1. Local Soil and Waste-Simulant Compositions in Mass Fractions

\begin{tabular}{|l|c|c|}
\hline & Soil & $\begin{array}{c}\text { Waste } \\
\text { Simulant }\end{array}$ \\
\hline $\mathrm{Al}_{2} \mathrm{O}_{3}$ & 0.1396 & 0.0188 \\
\hline $\mathrm{CaO}$ & 0.0550 & \\
\hline $\mathrm{Cl}$ & & 0.0090 \\
\hline $\mathrm{Cr}_{2} \mathrm{O}_{3}$ & & 0.0046 \\
\hline $\mathrm{F}$ & & 0.0035 \\
\hline $\mathrm{Fe}_{2} \mathrm{O}_{3}$ & 0.0928 & \\
\hline $\mathrm{K}_{2} \mathrm{O}$ & 0.0248 & 0.0034 \\
\hline $\mathrm{MgO}$ & 0.0143 & \\
\hline $\mathrm{Na}_{2} \mathrm{O}$ & 0.0321 & 0.8983 \\
\hline $\mathrm{P}_{2} \mathrm{O}_{5}$ & 0.0029 & 0.0202 \\
\hline $\mathrm{ReO}_{2}{ }^{(a)}$ & & 0.0001 \\
\hline $\mathrm{SO}_{3}$ & & 0.0418 \\
\hline $\mathrm{SiO}_{2}$ & 0.6242 & \\
\hline $\mathrm{TiO}_{2}$ & 0.0143 & \\
\hline $\mathrm{ZrO}_{2}$ & 0.0000 & \\
\hline $\mathrm{Total}^{2}$ & 1.0000 & 1.0000 \\
\hline
\end{tabular}

Table 3.2. Compositions of AMBG Test Glasses in Mass Fractions

\begin{tabular}{|l|c|c|c|c|c|c|c|c|}
\hline & $\mathbf{0 1}$ & $\mathbf{0 2}$ & $\mathbf{0 3}$ & $\mathbf{0 4}$ & $\mathbf{0 5}$ & $\mathbf{0 6}$ & $\mathbf{0 7}$ & $\mathbf{0 8}$ \\
\hline $\mathrm{Al}_{2} \mathrm{O}_{3}$ & 0.1204 & 0.1162 & 0.1120 & 0.1078 & 0.1090 & 0.1090 & 0.1048 & 0.1048 \\
\hline $\mathrm{B}_{2} \mathrm{O}_{3}$ & 0.0000 & 0.0000 & 0.0000 & 0.0000 & 0.0000 & 0.0250 & 0.0000 & 0.0250 \\
\hline $\mathrm{CaO}$ & 0.0462 & 0.0443 & 0.0424 & 0.0405 & 0.0415 & 0.0415 & 0.0396 & 0.0396 \\
\hline $\mathrm{Cl}$ & 0.0014 & 0.0017 & 0.0021 & 0.0024 & 0.0018 & 0.0018 & 0.0021 & 0.0021 \\
\hline $\mathrm{Cr}_{2} \mathrm{O}_{3}$ & 0.0007 & 0.0009 & 0.0011 & 0.0012 & 0.0009 & 0.0009 & 0.0011 & 0.0011 \\
\hline $\mathrm{F}$ & 0.0006 & 0.0007 & 0.0008 & 0.0009 & 0.0007 & 0.0007 & 0.0008 & 0.0008 \\
\hline $\mathrm{Fe}_{2} \mathrm{O}_{3}$ & 0.0780 & 0.0748 & 0.0716 & 0.0684 & 0.0700 & 0.0700 & 0.0668 & 0.0668 \\
\hline $\mathrm{K}_{2} \mathrm{O}$ & 0.0214 & 0.0207 & 0.0199 & 0.0192 & 0.0194 & 0.0194 & 0.0186 & 0.0186 \\
\hline $\mathrm{MgO}$ & 0.0120 & 0.0115 & 0.0110 & 0.0105 & 0.0108 & 0.0108 & 0.0103 & 0.0103 \\
\hline $\mathrm{Na}_{2} \mathrm{O}$ & 0.1700 & 0.2000 & 0.2300 & 0.2600 & 0.2000 & 0.2000 & 0.2300 & 0.2300 \\
\hline $\mathrm{P}_{2} \mathrm{O}_{5}$ & 0.0057 & 0.0063 & 0.0069 & 0.0075 & 0.0061 & 0.0061 & 0.0067 & 0.0067 \\
\hline $\mathrm{ReO}_{2}$ & 0.0001 & 0.0001 & 0.0001 & 0.0001 & 0.0001 & 0.0001 & 0.0001 & 0.0001 \\
\hline $\mathrm{SiO}_{2}$ & 0.5248 & 0.5032 & 0.4816 & 0.4600 & 0.4707 & 0.4707 & 0.4492 & 0.4492 \\
\hline $\mathrm{SO}_{3}$ & 0.0067 & 0.0081 & 0.0095 & 0.0110 & 0.0082 & 0.0082 & 0.0096 & 0.0096 \\
\hline $\mathrm{TiO}_{2}$ & 0.0120 & 0.0115 & 0.0110 & 0.0105 & 0.0108 & 0.0108 & 0.0103 & 0.0103 \\
\hline $\mathrm{ZrO}_{2}$ & 0.0000 & 0.0000 & 0.0000 & 0.0000 & 0.0500 & 0.0250 & 0.0500 & 0.0250 \\
\hline $\mathrm{Total}^{2}$ & 1.0000 & 1.0000 & 1.0000 & 1.0000 & 1.0000 & 1.0000 & 1.0000 & 1.0000 \\
\hline Soil & 0.841 & 0.806 & 0.772 & 0.737 & 0.754 & 0.754 & 0.720 & 0.720 \\
\hline aaste & 0.159 & 0.194 & 0.228 & 0.263 & 0.196 & 0.196 & 0.230 & 0.230 \\
\hline Additive & & & & & 0.050 & 0.050 & 0.050 & 0.050 \\
\hline Melting T( $\left.{ }^{\circ} \mathrm{C}\right)$ & 1512 & 1429 & 1349 & 1271 & 1405 & 1357 & 1332 & 1283 \\
\hline
\end{tabular}

(a) GDL-GBM = Glass Development Laboratory-Glass Batching and Melting. 
Table 3.2. Compositions of AMBG Test Glasses in Mass Fractions (cont.)

\begin{tabular}{|l|c|c|c|c|c|c|c|c||}
\hline & $\mathbf{0 9}$ & $\mathbf{1 0}$ & $\mathbf{1 1}$ & $\mathbf{1 2}$ & $\mathbf{1 3}$ & $\mathbf{1 4}$ & $\mathbf{1 5}$ & $\mathbf{1 6}$ \\
\hline $\mathrm{Al}_{2} \mathrm{O}_{3}$ & 0.0848 & 0.1248 & 0.1080 & 0.1016 & 0.0989 & 0.0946 & 0.0946 & 0.0988 \\
\hline $\mathrm{B}_{2} \mathrm{O}_{3}$ & 0.0000 & 0.0000 & 0.0000 & 0.0000 & 0.0500 & 0.0500 & 0.0500 & 0.0500 \\
\hline $\mathrm{CaO}$ & 0.0409 & 0.0383 & 0.0408 & 0.0383 & 0.0375 & 0.0358 & 0.0358 & 0.0377 \\
\hline $\mathrm{Cl}$ & 0.0021 & 0.0021 & 0.0021 & 0.0021 & 0.0018 & 0.0018 & 0.0018 & 0.0015 \\
\hline $\mathrm{Cr}_{2} \mathrm{O}_{3}$ & 0.0011 & 0.0011 & 0.0011 & 0.0011 & 0.0009 & 0.0009 & 0.0009 & 0.0008 \\
\hline $\mathrm{F}$ & 0.0008 & 0.0008 & 0.0008 & 0.0008 & 0.0007 & 0.0007 & 0.0007 & 0.0006 \\
\hline $\mathrm{Fe}_{2} \mathrm{O}_{3}$ & 0.0690 & 0.0645 & 0.0468 & 0.0868 & 0.0633 & 0.0604 & 0.0604 & 0.0636 \\
\hline $\mathrm{K}_{2} \mathrm{O}$ & 0.0192 & 0.0180 & 0.0192 & 0.0181 & 0.0176 & 0.0168 & 0.0168 & 0.0176 \\
\hline $\mathrm{La}_{2} \mathrm{O}_{3}$ & & & & & & & 0200 & \\
\hline $\mathrm{MgO}$ & 0.0106 & 0.0099 & 0.0106 & 0.0100 & 0.0097 & 0.0093 & 0.0093 & 0.0098 \\
\hline $\mathrm{Na}_{2} \mathrm{O}$ & 0.2300 & 0.2300 & 0.2300 & 0.2300 & 0.2000 & 0.2000 & 0.2000 & 0.1700 \\
\hline $\mathrm{P}_{2} \mathrm{O}_{5}$ & 0.0068 & 0.0067 & 0.0068 & 0.0067 & 0.0060 & 0.0059 & 0.0159 & 0.0053 \\
\hline $\mathrm{ReO}_{2}$ & 0.0001 & 0.0001 & 0.0001 & 0.0001 & 0.0001 & 0.0001 & 0.0001 & 0.0001 \\
\hline $\mathrm{SiO}_{2}$ & 0.4644 & 0.4341 & 0.4635 & 0.4347 & 0.4255 & 0.4061 & 0.4061 & 0.4575 \\
\hline $\mathrm{SO}_{3}$ & 0.0096 & 0.0097 & 0.0096 & 0.0097 & 0.0083 & 0.0083 & 0.0083 & 0.0069 \\
\hline $\mathrm{TiO}_{2}$ & 0.0106 & 0.0099 & 0.0106 & 0.0100 & 0.0097 & 0.0093 & 0.0193 & 0.0098 \\
\hline $\mathrm{ZrO}_{2}$ & 0.0500 & 0.0500 & 0.0500 & 0.0500 & 0.0700 & 0.1000 & 0.0600 & 0.0700 \\
\hline $\mathrm{Total}^{\mathrm{Soil}}$ & 1.0000 & 1.0000 & 1.0000 & 1.0000 & 1.0000 & 1.0000 & 1.0000 & 1.0000 \\
\hline $\mathrm{Waste}$ & 0.721 & 0.719 & 0.720 & 0.719 & 0.682 & 0.651 & 0.651 & 0.685 \\
\hline $\mathrm{Additive}$ & 0.229 & 0.231 & 0.230 & 0.231 & 0.198 & 0.199 & 0.199 & 0.165 \\
\left.\hline${\mathrm{Melting} \mathrm{T}\left({ }^{\circ} \mathrm{C}\right)}\right)$ & 0.050 & 0.050 & 0.050 & 0.050 & 0.120 & 0.150 & 0.150 & 0.150 \\
\hline & & & & & & & & \\
\hline
\end{tabular}

Table 3.3. Source Chemicals

\begin{tabular}{|c|c|c|c|}
\hline Component & Manufacturer & $\begin{array}{c}\text { Lot } \\
\text { Number }\end{array}$ & $\begin{array}{c}\text { LOD }^{(a)} \\
(\text { mass \%) }\end{array}$ \\
\hline $\mathrm{Al}_{2} \mathrm{O}_{3}$ & Fisher & 006627 & 0.000 \\
\hline $\mathrm{H}_{3} \mathrm{BO}_{3}$ & Noah & $20032 / 2.1$ & $44.26^{(\mathrm{b})}$ \\
\hline $\mathrm{Cr}_{2} \mathrm{O}_{3}$ & Fisher & 851377 & 0.000 \\
\hline $\mathrm{FeO}$ & Alfa products & C03N06 & 0.208 \\
\hline $\mathrm{K}_{2} \mathrm{CO}_{3}$ & Fisher & 005661 & 0.763 \\
\hline $\mathrm{Na}_{2} \mathrm{CO}_{3}$ & Fisher & 025436 & 0.000 \\
\hline $\mathrm{SiO}_{2}$ & Fisher & 016166 & 0.000 \\
\hline $\mathrm{ZrO}_{2}$ & Noah & $18151 / 1.1$ & 0.000 \\
\hline $\mathrm{CaCO}_{3}$ & Fisher & 007112 & 0.372 \\
\hline $\mathrm{NaCl}$ & Sigma & $74 \mathrm{H} 1061$ & 0.272 \\
\hline $\mathrm{NaF}$ & Mallinckrodt & WBXZ & 0.000 \\
\hline $\mathrm{MgO}$ & Fisher & 700694 & 0.904 \\
\hline $\mathrm{NaPO}_{3}$ & J.T. Baker & X09610 & 0.371 \\
\hline $\mathrm{ReO}_{2}$ & Alfa products & G23J09 & 0.000 \\
\hline $\mathrm{Na}_{2} \mathrm{SO}_{4}$ & J.T. Baker & 22102 & 0.086 \\
\hline $\mathrm{TiO}_{2}$ & J.T. Baker & 525355 & 0.273 \\
\hline
\end{tabular}

Batch chemicals were weighed to within $\pm 1 \%$ relative precision. Batches were mixed in an agate milling chamber in the Angstrom milling machine for approximately 4-min and melted in Pt-10\%Rh crucibles. The minimum temperature at which melting began is indicated in Table 3.2 (a temperature at which the viscosity estimated using the model by Vienna et al. 2002 was $5 \mathrm{~Pa} \cdot \mathrm{s}$ or higher). The crucible was covered with a lid with a hole through which argon was introduced to prevent the oxidation of $\mathrm{FeO}$. After $1 \mathrm{~h}$ of melting, the glass was quenched on a stainless steel plate. The glass was homogenized by grinding into a fine powder in a tungsten-carbide milling chamber in the Angstrom milling machine for $4 \mathrm{~min}$. The ground glass was remelted in a Pt- $10 \% \mathrm{Rh}$ crucible with a tight lid under argon at the same temperature as the first melt. If the first-melt glass contained crystals, the melting temperature of the second melt

was raised by $50^{\circ} \mathrm{C}$. The melt was poured to fill a small stainless steel mold $(15 \times 15 \times 20 \mathrm{~mm}[0.6 \times 0.6 \times 0.8$ in.]) heated on a hot plate for the VHT sample of the quenched glass, and a larger platinum mold $(25.4 \times 25.4 \times 88.9 \mathrm{~mm}[1 \times 1 \times 3.5 \mathrm{in}]$.$) for the \mathrm{SC}$ heat-treatment sample. The remaining glass melt was 
poured onto a clean stainless steel quench plate. The small mold was transferred into a preheated oven for annealing.

Each composition underwent two extreme heat treatments: a rapid cooling and a slow cooling (SC, which represents the slowest cooling that glass experiences during ICV at full scale). SC schedule is defined in Table 3.4. The starting temperature for $\mathrm{SC}$ heat treatment was determined as the temperature at which the predicted $\eta=10 \mathrm{~Pa} \cdot \mathrm{s}$. Glass samples with these two temperature histories (i.e., rapid cooling and SC) were tested for key properties (i.e., VHT, PCT, TCLP, $\rho$, and crystal-phase identification and quantification).

\subsubsection{Product Consistency Test}

Table 3.5 shows the elemental releases of six major elements by ICP-

Table 3.4. Slow Cooling Schedule

\begin{tabular}{|c|c|}
\hline $\begin{array}{c}\text { Time } \\
(\mathbf{h})\end{array}$ & $\begin{array}{c}\text { Temperature } \\
\left({ }^{\circ} \mathbf{C}\right)\end{array}$ \\
\hline 0 & 1600 \\
\hline 6 & 1400 \\
\hline 10 & 1300 \\
\hline 24 & 1080 \\
\hline 48 & 880 \\
\hline 72 & 720 \\
\hline 96 & 600 \\
\hline 120 & 500 \\
\hline
\end{tabular}
AES. All glasses except for AMBG-04 passed the $2 \mathrm{~g} / \mathrm{m}^{2}$ constraint for PCT for both quenched and SC samples; AMBG-13 through 16 passed by nearly an order of magnitude. Figure 3.1 and Figure 3.2 compare the PCT normalized releases of $\mathrm{Al}, \mathrm{B}, \mathrm{K}$, and $\mathrm{Si}$ with the normalized $\mathrm{Na}$ release for quenched and SC-treated samples, respectively, showing that the normalized release of $\mathrm{Na}$ is the most conservative indication of glass dissolution in PCT conditions. Figure 3.3 shows the effect of SC treatment on the PCT Na normalized release for AMBG glasses, indicating that the slow cooling upon SC treatment had no adverse effect on the PCT response; SC treatment results in slightly lower PCT releases as compared to quenched glass.

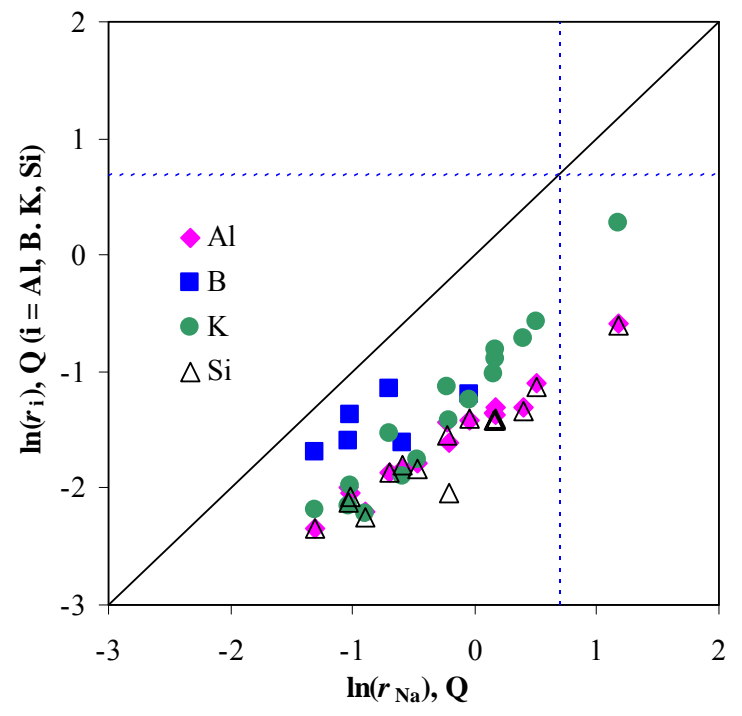

Figure 3.1. PCT Releases (in $\mathrm{g} / \mathrm{m}^{2}$ ) versus Na Release (Quenched Samples)

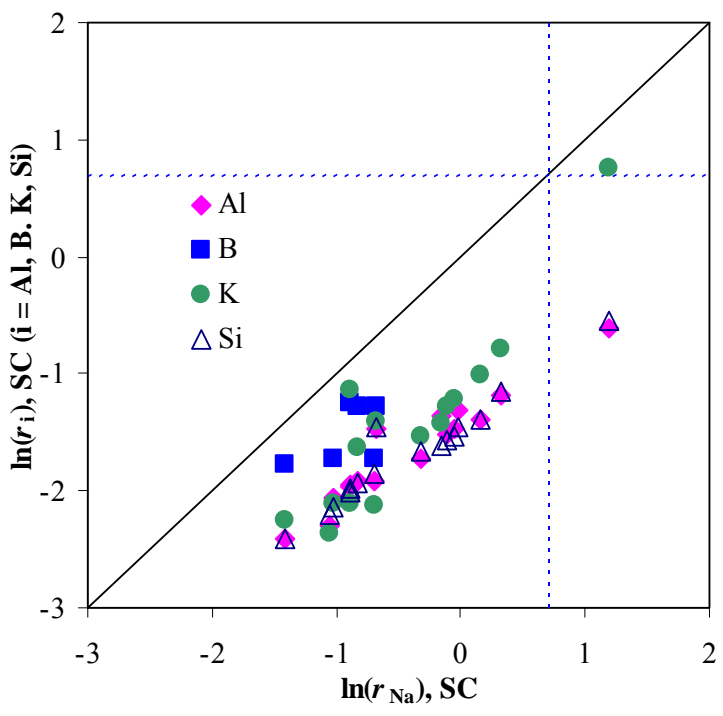

Figure 3.2. PCT Releases (in $\mathrm{g} / \mathrm{m}^{2}$ ) versus $\mathrm{Na}$ Release (SC Samples) 
Table 3.5. PCT Normalized Releases in $\mathrm{g} / \mathrm{m}^{2}$ from AMBG Glasses

\begin{tabular}{||c|c|c|c|c|c|c||}
\hline \multicolumn{7}{|c|}{ Quenched Glasses } \\
\hline & $\boldsymbol{r}_{\mathbf{N a}}$ & $\boldsymbol{r}_{\mathbf{A l}}$ & $\boldsymbol{r}_{\mathbf{B}}$ & $\boldsymbol{r}_{\mathbf{C a}}$ & $\boldsymbol{r}_{\mathbf{K}}$ & $\boldsymbol{r}_{\mathbf{S i}}$ \\
\hline AMBG-01-Q & 0.407 & 0.111 & & 0.009 & 0.109 & 0.105 \\
\hline AMBG-02-Q & 0.816 & 0.199 & & 0.013 & 0.241 & 0.129 \\
\hline AMBG-03-Q & 1.650 & 0.332 & & 0.007 & 0.569 & 0.324 \\
\hline AMBG-04-Q & 3.230 & 0.556 & & 0.009 & 1.313 & 0.549 \\
\hline AMBG-05-Q & 0.627 & 0.167 & & 0.011 & 0.174 & 0.160 \\
\hline AMBG-06-Q & 0.553 & 0.159 & 0.200 & 0.015 & 0.150 & 0.165 \\
\hline AMBG-07-Q & 1.168 & 0.257 & & 0.010 & 0.361 & 0.243 \\
\hline AMBG-08-Q & 0.952 & 0.241 & 0.303 & 0.008 & 0.290 & 0.248 \\
\hline AMBG-09-Q & 1.497 & 0.270 & & 0.010 & 0.485 & 0.263 \\
\hline AMBG-10-Q & 0.804 & 0.238 & & 0.005 & 0.321 & 0.212 \\
\hline AMBG-11-Q & 1.194 & 0.253 & & 0.004 & 0.410 & 0.242 \\
\hline AMBG-12-Q & 1.194 & 0.270 & & 0.008 & 0.441 & 0.246 \\
\hline AMBG-13-Q & 0.364 & 0.129 & 0.253 & 0.008 & 0.138 & 0.126 \\
\hline AMBG-14-Q & 0.357 & 0.137 & 0.202 & 0.008 & 0.116 & 0.120 \\
\hline AMBG-15-Q & 0.499 & 0.155 & 0.316 & 0.009 & 0.217 & 0.154 \\
\hline AMBG-16-Q & 0.269 & 0.095 & 0.184 & 0.008 & 0.112 & 0.096 \\
\hline \hline \multicolumn{7}{|c|}{ SC-Treated Glasses } \\
\hline AMBG-01-C & 0.349 & 0.100 & & 0.008 & 0.094 & 0.111 \\
\hline AMBG-02-C & 0.730 & 0.178 & & 0.010 & 0.217 & 0.189 \\
\hline AMBG-03-C & 1.384 & 0.305 & & 0.007 & 0.455 & 0.315 \\
\hline AMBG-04-C & 3.291 & 0.545 & & & 2.143 & 0.577 \\
\hline AMBG-05-C & 0.408 & 0.143 & & 0.008 & 0.121 & 0.137 \\
\hline AMBG-06-C & 0.502 & 0.147 & 0.178 & 0.005 & 0.119 & 0.157 \\
\hline AMBG-07-C & 0.953 & 0.230 & & 0.005 & 0.294 & 0.215 \\
\hline AMBG-08-C & 0.504 & 0.228 & 0.278 & 0.007 & 0.246 & 0.233 \\
\hline AMBG-09-C & 1.173 & 0.250 & & 0.010 & 0.362 & 0.249 \\
\hline AMBG-10-C & 0.862 & 0.259 & & 0.006 & 0.241 & 0.198 \\
\hline AMBG-11-C & 0.900 & 0.219 & & 0.007 & 0.280 & 0.208 \\
\hline AMBG-12-C & 0.979 & 0.268 & & 0.012 & & 0.234 \\
\hline AMBG-13-C & 0.410 & 0.140 & 0.289 & 0.028 & 0.321 & 0.134 \\
\hline AMBG-14-C & 0.361 & 0.128 & 0.178 & 0.008 & 0.121 & 0.117 \\
\hline AMBG-15-C & 0.438 & 0.147 & 0.279 & 0.008 & 0.196 & 0.145 \\
\hline AMBG-16-C & 0.245 & 0.090 & 0.169 & 0.008 & 0.105 & 0.090 \\
\hline \hline
\end{tabular}




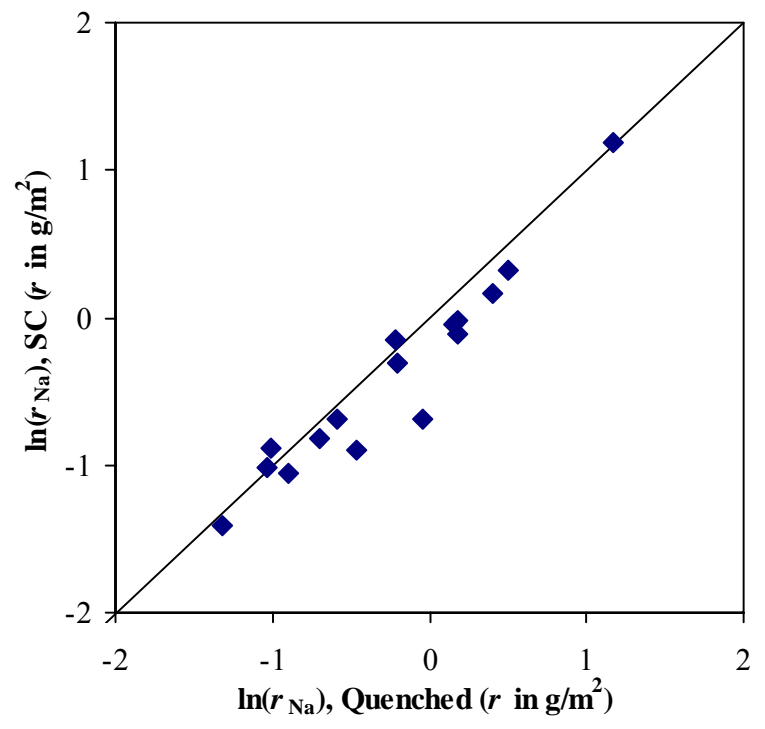

\subsubsection{Vapor Hydration Test}

Table 3.6 lists the $200^{\circ} \mathrm{C}$ VHT responses of AMBG glasses. Not included in the table are both quenched and SC-treated samples of AMBG-01 to 04, 07, 08, and 10 to 12, and quenched 06 , which completely corroded during the 14-day tests. Glasses AMBG-13, 14 , and 16 had average corrosion rates lower than $50 \mathrm{~g} /\left(\mathrm{m}^{2} \cdot \mathrm{d}\right)$ for quenched and SC samples and for all durations tested.

Figure 3.3. Effect of SC Treatment on Normalized Na Release

Table 3.6. $200^{\circ} \mathrm{C}$-VHT Response of AMBG Glasses

\begin{tabular}{|c|c|c|c|c|c|c|c|}
\hline Test Number & $\begin{array}{c}\text { Duration } \\
\text { (days) }\end{array}$ & $\begin{array}{c}m \\
\left(\mathrm{~g} / \mathrm{m}^{2}\right)\end{array}$ & $\begin{array}{c}r_{\mathrm{a}} \\
\left(\mathrm{g} /\left[\mathrm{m}^{2} \cdot \mathrm{d}\right]\right)\end{array}$ & Test Number & $\begin{array}{c}\text { Duration } \\
\text { (days) }\end{array}$ & $\begin{array}{c}m \\
\left(g / m^{2}\right)\end{array}$ & $\begin{array}{c}r_{\mathrm{a}} \\
\left(\mathrm{g} /\left[\mathrm{m}^{2} \cdot \mathrm{d}\right]\right)\end{array}$ \\
\hline AMBG-05-Q & 6.9 & 122.2 & 17.59 & AMBG-13-SC & 13.9 & 5.2 & 0.37 \\
\hline AMBG-05-Q & 13.9 & 1193.3 & 85.75 & AMBG-13-SC & 28.0 & 96.9 & 3.46 \\
\hline AMBG-05-Q & 18.1 & 1449.9 & 80.25 & AMBG-14-Q & 6.9 & 4.0 & 0.58 \\
\hline AMBG-05-Q & 21.0 & 1875.2 & 89.12 & AMBG-14(2)-Q & 7.2 & 5.5 & 0.76 \\
\hline AMBG-05-SC & 6.9 & 118.6 & 17.12 & AMBG-14-Q & 14.0 & 0.0 & 0.00 \\
\hline AMBG-05-SC & 13.9 & 482.9 & 34.68 & AMBG-14(2)-Q & 14.2 & 9.2 & 0.65 \\
\hline AMBG-05-SC & 18.1 & 952.3 & 52.71 & AMBG-14(2)-Q & 28.1 & 2.7 & 0.10 \\
\hline AMBG-05-SC & 21.0 & 1545.7 & 73.53 & AMBG-14(2)-SC & 7.0 & 4.0 & 0.58 \\
\hline AMBG-06-Q & 6.9 & 141.8 & 20.42 & AMBG-14(2)-SC & 14.0 & 5.3 & 0.38 \\
\hline AMBG-06-Q & 9.9 & 1369.5 & 137.83 & AMBG-14(2)-SC & 28.0 & 1.3 & 0.05 \\
\hline AMBG-06-SC & 6.9 & 452.4 & 65.30 & AMBG-15-Q & 6.9 & 401.1 & 57.74 \\
\hline AMBG-06-SC & 9.9 & 1276.8 & 128.76 & AMBG-15-Q & 10.0 & 684.1 & 68.60 \\
\hline AMBG-06-SC & 13.9 & 1715.8 & 123.24 & AMBG-15-Q & 14.0 & 1050.2 & 75.01 \\
\hline AMBG-09-Q & 6.9 & 87.4 & 12.59 & AMBG-15-SC & 7.0 & 480.4 & 68.78 \\
\hline AMBG-09-Q & 9.9 & 492.2 & 49.54 & AMBG-15-SC & 13.9 & 588.2 & 42.17 \\
\hline AMBG-09-Q & 13.9 & 1655.6 & 118.98 & AMBG-15-SC & 21.0 & 630.7 & 30.04 \\
\hline AMBG-09-SC & 6.9 & 111.6 & 16.11 & AMBG-16-Q & 7.1 & 2.7 & 0.38 \\
\hline AMBG-09-SC & 9.9 & 1056.5 & 106.54 & AMBG-16-Q & 14.1 & 3.9 & 0.28 \\
\hline AMBG-09-SC & 13.9 & 1901.3 & 136.66 & AMBG-16-Q & 28.1 & 9.2 & 0.33 \\
\hline AMBG-13-Q & 6.9 & 6.7 & 0.96 & AMBG-16-SC & 7.0 & 42.2 & 6.05 \\
\hline AMBG-13-Q & 14.0 & 13.5 & 0.97 & AMBG-16-SC & 13.9 & 14.2 & 1.02 \\
\hline AMBG-13-Q & 28.1 & 223.3 & 7.96 & AMBG-16-SC & 28.0 & 2.7 & 0.10 \\
\hline AMBG-13-SC & 7.0 & 44.6 & 6.39 & & & & \\
\hline
\end{tabular}


The VHT responses of these three glasses are compared to data from literature in Figure 3.4 showing that the VHT response of typical ICV glass samples performed well below those used to set the constraint on acceptable glasses (LAW-A33), better than the glass that formed the basis of the 2001 PA (LAW-

ABP1-Mann et al. 2001), and at least as well as typical WTP glasses (Muller et al. 2001). Figure 3.5 compares average corrosion rates of quenched and SC-treated samples (the large scatter for the glasses with low corrosion rates is exaggerated by using a logarithmic scale).

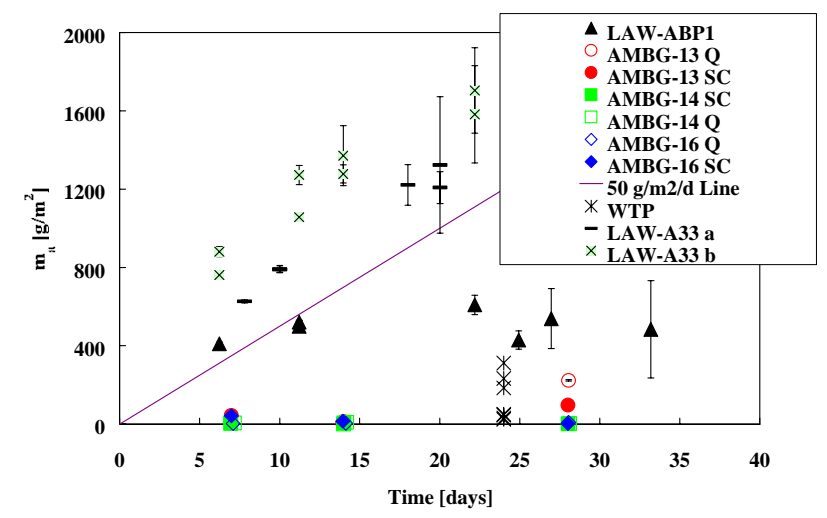

Figure 3.4. Comparison of VHT Responses for AMBG-13, -14, and -16 with those from LAW-A33, LAW-ABP1, and Typical WTP Glasses

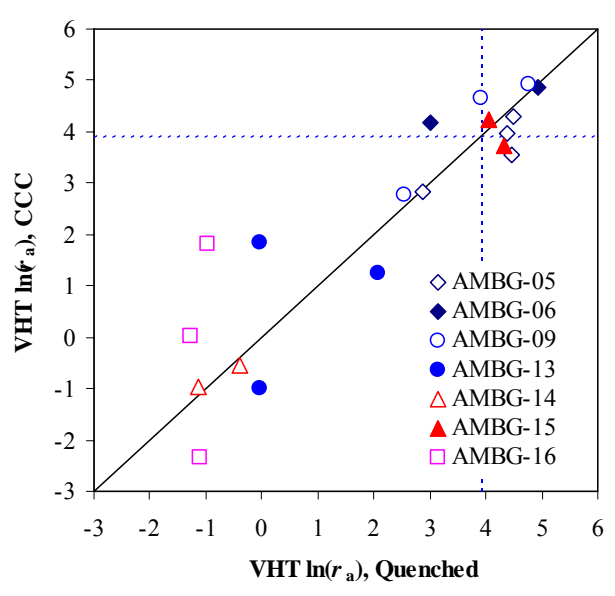

Figure 3.5. Comparison of VHT Corrosion Rates in Quenched and SC Samples
The crystalline alteration products were identified for a selected set of samples by XRD. These phases are listed in Table 3.7. It should be noted that the phases were identified by crystal structure and not chemical analyses, so, other minerals or compositions with the structure of those identified may be present. For example, $\mathrm{SrZrO}_{3}$ is unlikely while $\mathrm{CaZrO}_{3}$ may have formed.

The VHT response is the most limiting property for the ICV product with Hanford LAW. ASCM-04 glass was prepared in three different oxidation-reduction states (Table 3.8). Glass AMOG-01 was heat treated under Ar with 2.84 vol\% $\mathrm{H}_{2}$, glass AMOG-02 under a $\mathrm{CO}_{2}-\mathrm{CO}$ gas mixture, and glass AMOG-03 under pure oxygen. To control the atmosphere, glasses were melted in a sealed alumina tube with openings for inlet and outlet gas tubes and for the thermocouple.

Figure 3.6 shows that the VHT mass loss decreased linearly with the increasing fraction of Fe(II). Table 3.9 lists the measured glass-redox values for AMOG glasses and the VHT mass losses at 7-, 14-, and 28- 
days. This result implies that Fe(III) tends to increase the VHT mass loss - an effect similar to that of Al. Both $\mathrm{Al}$ and $\mathrm{Fe}(\mathrm{III})$ are glass formers in high-alkali borosilicate glasses and both need alkali ions for charge compensation. Both $\mathrm{Al}$ and $\mathrm{Fe}(\mathrm{III})$ decrease the initial rate of corrosion, but hasten the transition to the final stage of corrosion. These corrosion stages were identified by Vienna et al. (2001) who also showed that the final corrosion rate $\left(r_{\infty}\right)$ decreased with increasing $\mathrm{Fe}(\mathrm{II}) / \mathrm{Fe}$ fraction. They measured this effect of $\mathrm{Fe}(\mathrm{II}) / \mathrm{Fe}$ on $r_{\infty}$ for only one glass and no attempt was made to demonstrate that it applies to more than one composition. Longer test duration (beyond the deadline for this report) would be needed to establish the final corrosion rate $\left(r_{\infty}\right)$ for AMOG glasses.

Table 3.8. Fe Sources and Gas Compositions for Glasses with Varied Iron Redox Melted at $1270^{\circ} \mathrm{C}$

\begin{tabular}{||l|c|c|c||}
\hline & ID & Iron Source & Atmosphere \\
\hline Reduced & AMOG-01 & $\mathrm{FeO}$ & $2.84 \% \mathrm{H}_{2}$ in $\mathrm{Ar}$ \\
\hline Half-reduced & AMOG-02 & $0.763 \mathrm{Fe}_{3} \mathrm{O}_{4}+0.237 \mathrm{FeO}$ & $3.26 \% \mathrm{CO}$ in $\mathrm{CO}_{2}$ \\
\hline Oxidized & AMOG-03 & $\mathrm{Fe}_{2} \mathrm{O}_{3}$ & $\mathrm{O}_{2}$ \\
\hline
\end{tabular}

Table 3.9. Measured Redox Ratio for AMOG Samples and $200^{\circ} \mathrm{C}$ VHT Mass Losses in $\mathrm{g} / \mathrm{m}^{2}$

\begin{tabular}{||c|c|c|r|c||}
\hline Glass ID & Fe(II)/Fe & 7 days & 14 days & 28 days \\
\hline AMOG-01 & 0.87 & 4.1 & 45.4 & 150.7 \\
\hline AMOG-02 & 0.40 & 2.7 & 149.3 & 303.7 \\
\hline AMOG-03 & 0.00 & 2.7 & 159.3 & 564.5 \\
\hline
\end{tabular}

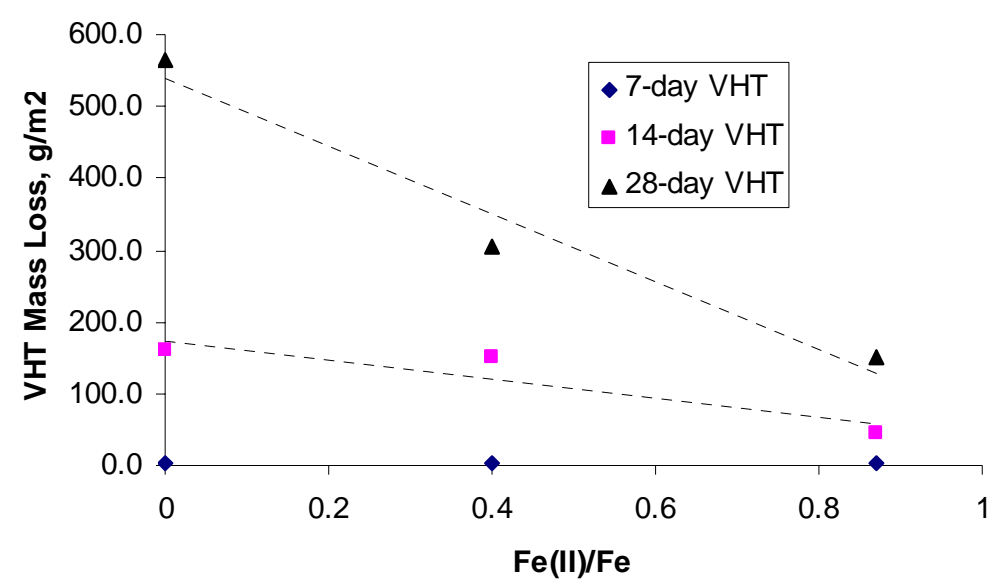

Figure 3.6. VHT Effect of the Oxidation-Reduction State of Iron on the VHT Mass Loss for AMOG Glasses 


\subsubsection{Toxicity Characteristic Leach Procedure}

The $\mathrm{Cr}$ is the only regulated component in AMBG glasses (Table 3.10). The B release is included in Table 3.10 because the normalized $\mathrm{B}$ release $\left(r_{\mathrm{B}}\right)$ is used as a representative measure of glass dissolution in the TCLP condition (Kim and Vienna 2002). As expected from low $\mathrm{Cr}_{2} \mathrm{O}_{3}$ in glass ( 0.09 to 0.12 mass\%), all the glasses, even the glass with 26 mass $\% \mathrm{Na}_{2} \mathrm{O}$ (AMBG-04), exhibited $\mathrm{Cr}$ release well below the EPA UTS limit of $0.6 \mathrm{mg} / \mathrm{L}$.
Table 3.10. TCLP Responses (in $\mathrm{mg} / \mathrm{L}$ ) of Selected AMBG Glasses

\begin{tabular}{||c|c|c|c|c|c||}
\hline & $\begin{array}{c}\text { UTS } \\
\text { Limit }\end{array}$ & 04-Q & 10-SC & 13-Q & 13-SC \\
\hline B & NA & NA & NA & 0.43 & 0.52 \\
\hline $\mathrm{Cr}$ & 0.6 & 0.0071 & 0.07 & 0.011 & 0.0059 \\
\hline $\begin{array}{l}\text { The italicized values in highlighted cells are } \\
\text { estimated results because they are below the } \\
\text { reporting limits (0.5 and } 0.25 \mathrm{mg} / \mathrm{L} \text { for B and } \mathrm{Cr} \\
\text { respectively). }\end{array}$
\end{tabular}

The relative performance of different glasses under TCLP leach conditions can be properly compared based on normalized releases, generally normalized B release. Figure 3.7 shows that the ICV glasses had lower normalized B releases than typical WTP LAW glasses (Muller et al. 2001, Muller and Pegg 1998, and Kot and Pegg 2001). This result implies that the ICV glasses can contain higher concentrations of regulated toxic elements without failing the TCLP requirements.

\subsubsection{Viscosity and Electrical Conductivity}

Table 3.11 and Table 3.12 list the viscosity and electrical conductivity data for AMBG-13 and 16 glasses.

\subsubsection{Secondary Phase Identification}

Only glasses AMBG-04, 10, 12, and 14 showed signs of phase changes upon SC. In glasses AMBG-04, -10, and -12 , large numbers of crystals appeared at the $\mathrm{SC}$ sample surfaces within $\sim 1.5 \mathrm{~mm}$ from the Pt-glass interface. A few crystals, below the detection limit of $\mathrm{XRD}$, were seen in the bulk glass.

Table 3.13 lists mass fractions of crystalline phases identified in this layer: nepheline $\left[\mathrm{NaAlSiO}_{4}\right]$, combeite $\left[\mathrm{Na}_{4} \mathrm{Ca}_{4}\left(\mathrm{Si}_{6} \mathrm{O}_{18}\right)\right]$, and baddeleyite $\left[\mathrm{ZrO}_{2}\right]$. Figure 3.8 is an example SEM micrograph with nepheline and baddeleyite in the AMBG-10-SC sample. In AMBG-14 $\mathrm{ZrO}_{2}$ crystals partially settled to a $\sim 5-\mathrm{mm}$ layer at the crucible bottom. Mass fractions of a $\mathrm{ZrO}_{2}$ phase in the bulk sample and the crucible bottom area are also included in Table 3.13. Figure 3.9 shows optical micrographs of baddeleyite crystals formed and settled at the bottom of the SC-treated AMBG-14 glass. Figure 3.10 shows the SEM micrographs of baddeleyite crystals formed in SC-treated AMBG-14 glass. Dendritic growth patterns and agglomerates of irregular shapes with well defined edges were observed.

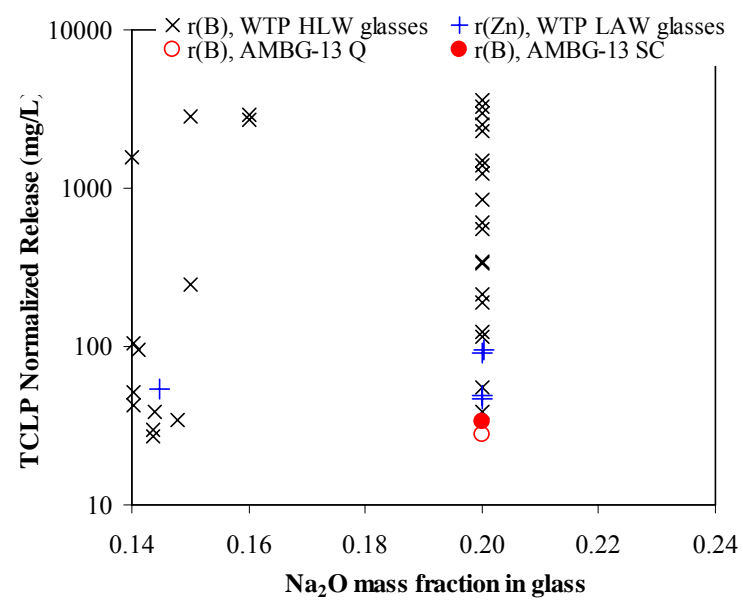

Figure 3.7. TCLP Normalized Releases for AMBG-13 and Typical WTP Glasses 
Table 3.11. Viscosity Results for AMBG-13 and 16 Glasses

\begin{tabular}{||c|c||c|c||}
\hline \hline \multicolumn{2}{||c||}{ AMBG-13 } & \multicolumn{2}{c||}{ AMBG-16 } \\
\hline$T\left({ }^{\circ} \mathrm{C}\right)$ & $\eta(\mathrm{Pa} \cdot \mathrm{s})$ & $T\left({ }^{\circ} \mathrm{C}\right)$ & $\eta(\mathrm{Pa} \cdot \mathrm{s})$ \\
\hline 1394 & 2.132 & 1343 & 5.439 \\
\hline 1344 & 3.646 & 1294 & 8.611 \\
\hline 1294 & 5.795 & 1244 & 14.871 \\
\hline 1344 & 3.680 & 1293 & 9.153 \\
\hline 1393 & 2.254 & 1343 & 6.124 \\
\hline 1343 & 3.840 & 1393 & 3.993 \\
\hline 1245 & 9.237 & 1343 & 6.437 \\
\hline 1195 & 16.039 & 1194 & 27.442 \\
\hline 1145 & 27.831 & 1144 & 47.481 \\
\hline 1095 & 51.116 & 1094 & 91.603 \\
\hline
\end{tabular}

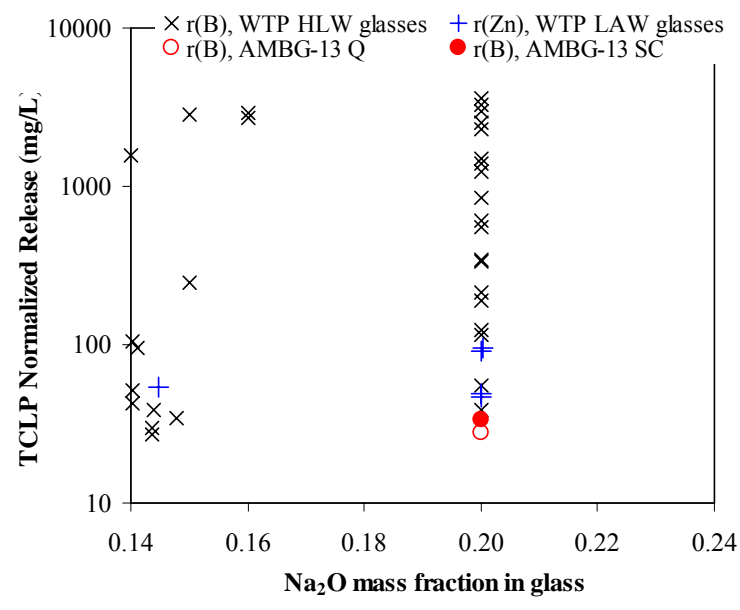

Figure 3.7. TCLP Normalized Releases for AMBG-13 and Typical WTP Glasses

Table 3.12. Electrical Conductivity Results for AMBG-13 and 16 Glasses

\begin{tabular}{|c|c|c|c|c|c|c|c|c|c|}
\hline \multicolumn{5}{|c|}{$\varepsilon$ of $A M B G-13(\mathrm{~S} / \mathrm{m})$} & \multicolumn{5}{|c|}{$\varepsilon$ of AMBG-16 (S/m) } \\
\hline$T\left({ }^{\circ} \mathbf{C}\right)$ & $100 \mathrm{~Hz}$ & $1 \mathrm{kHz}$ & $10 \mathrm{kHz}$ & $100 \mathrm{kHz}$ & $T\left({ }^{\circ} \mathrm{C}\right)$ & $100 \mathrm{~Hz}$ & $1 \mathrm{kHz}$ & $10 \mathrm{kHz}$ & $100 \mathrm{kHz}$ \\
\hline 1391 & 48.22 & 63.29 & 69.85 & 69.38 & 1392 & 38.57 & 39.75 & 39.55 & 39.35 \\
\hline 1294 & 36.11 & 47.15 & 51.97 & 51.88 & 1295 & 28.82 & 30.89 & 30.38 & 30.41 \\
\hline 1195 & 26.13 & 34.13 & 37.25 & 37.25 & 1195 & 20.65 & 23.05 & 22.33 & 22.25 \\
\hline 1095 & 18.10 & 23.47 & 25.23 & 25.27 & 1096 & 14.05 & 16.02 & 15.44 & 15.37 \\
\hline
\end{tabular}

Table 3.13. Crystalline Phases, in Mass\%, Determined by XRD in SC Samples within $~ 1.5-\mathrm{mm}$ at Pt-Glass Interface

\begin{tabular}{|c|c|c|c|}
\hline Glass & $\begin{array}{c}\text { Nepheline } \\
\left(\mathrm{NaAlSiO}_{4}\right) \\
\text { Mass\% }\end{array}$ & $\begin{array}{c}\text { Combeite } \\
{\left[\mathrm{Na}_{4} \mathrm{Ca}_{4}\left(\mathrm{Si}_{6} \mathrm{O}_{18}\right)\right]} \\
\mathrm{Mass} \%\end{array}$ & $\begin{array}{c}\text { Baddeleyite } \\
\left(\mathrm{ZrO}_{2}\right) \\
\text { Mass\% }\end{array}$ \\
\hline AMBG-04-SC, Pt-glass interface area & 1.15 & 0 & 0 \\
\hline AMBG-10-SC, Pt-glass interface area & 6.90 & 1.95 & 0.35 \\
\hline AMBG-12-SC, Pt-glass interface area & 0.35 & 0 & 0.27 \\
\hline AMBG-14B2-SC, bulk glass & 0 & 0 & 0.49 \\
\hline AMBG-14B2-SC, crucible bottom area & 0 & 0 & 5.36 \\
\hline
\end{tabular}

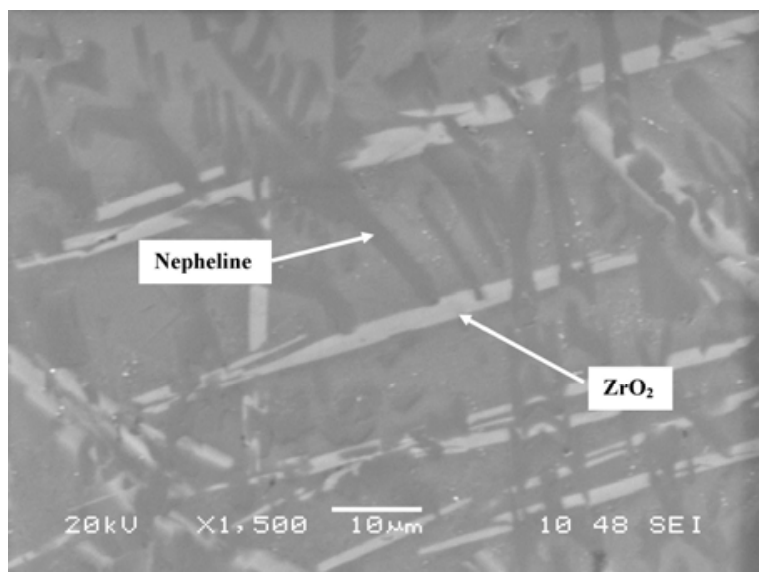

Figure 3.8. SEM

Micrograph Showing the Nepheline and Zirconia Crystals Formed at the Surface Layer of the SC Treated AMBG-10 Glass 


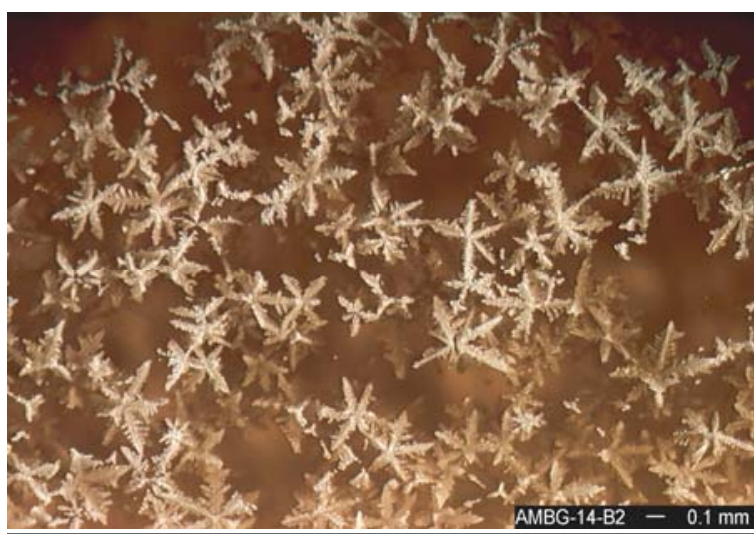

Figure 3.9. Optical

Micrographs Showing

Baddeleyite Crystals

Formed and Settled

at the Bottom of the SC

Treated AMBG-14 Glass

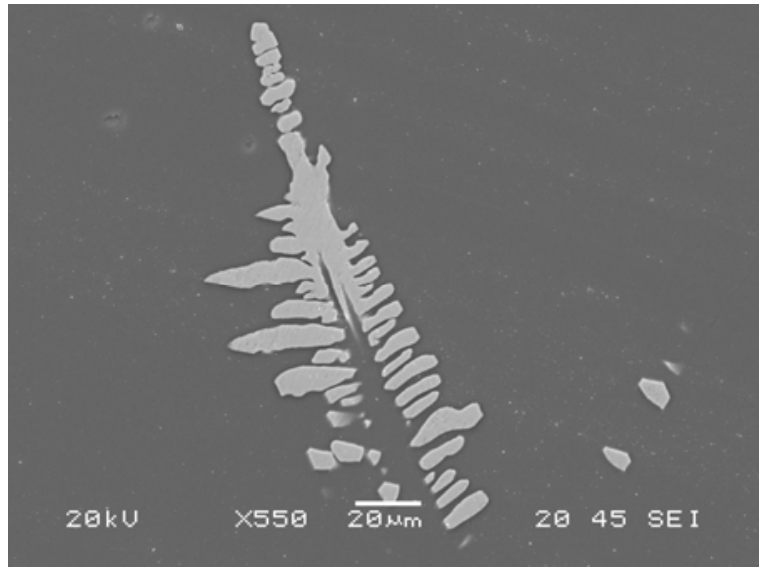

Figure 3.10. SEM

Micrographs of

Baddeleyite Crystals in

Slow-Cooled AMBG-14

Glass

\subsubsection{Baseline Glass Selection}

The glass with the best mix of properties is AMBG-13. This glass contains 20 mass $\% \mathrm{Na}_{2} \mathrm{O}$, has outstanding PCT and VHT responses, and does not contain crystals after SC heat treatment. This made it suitable for scale-up and radioactive testing of the ICV process.

\subsection{Simulant and Actual Waste Crucible Tests}

\subsubsection{Summary}

The LAW simulant was supplied by CH2M Hill Hanford Group. To determine its waste-loading bounds, five ASCM glasses with 17 to 24 mass $\% \mathrm{Na}_{2} \mathrm{O}$ were formulated around the AMBG-13 composition; ASCM-01 was the simulant version of AMBG-13. All five glasses passed the PCT and TCLP requirements with a large margin. The VHT requirement was met in four glasses (ASCM-01 through 04); ASCM-05 with target and measured 24 and 25.5 mass $\% \mathrm{Na}_{2} \mathrm{O}$ had $r_{a}>50 \mathrm{~g} / \mathrm{m}^{2} / \mathrm{d}$. Crystallization occurred in some ASCM glasses without any significant effect on their chemical durability. A radioactive version of AMBG-13 (ARCM-01) was prepared from actual Hanford LAW. No noticeable difference in measured properties was observed between these two glasses confirming the validity of data obtained from simulated LAW. The normalized Tc release from ARCM-01 was several time lower than the normalized $\mathrm{Na}$ or B release suggesting that there was no selective leaching of Tc under PCT condition. 


\subsubsection{Formulation and Fabrication}

To identify the effect of loading on the product quality for the baseline glass, five melts (Table 3.14) were formulated with simulated saltcake. One melt (ARCM-01) with an actual radioactive tank LAW had the same target composition as ASCM-01. All glasses had the same additive concentration of 7 mass $\% \mathrm{ZrO}_{2}$ and 5 mass $\% \mathrm{~B}_{2} \mathrm{O}_{3}$. In ASCM-02 glass, 3 mass $\% \mathrm{SiO}_{2}$ replaced an equivalent amount of soil in ASCM03 on a mass basis.

The actual AMEC-site soil and LAW simulant supplied by CHG (Rassat et al. 2003) were used (Table 3.1). The glass-oxide fractions that would result from the target mixtures of soil, LAW/LAW simulant, and additives are listed in Table 3.15.

Table 3.14. ASCM Target Glass Compositions in Mass\%

\begin{tabular}{||l|c|c|c|c|c||}
\hline \hline & ASCM-01 & ASCM-02 & ASCM-03 & ASCM-04 & ASCM-05 \\
\hline Soil & 68.2 & 68.5 & 71.6 & 65.9 & 63.6 \\
\hline Waste & 19.8 & 16.5 & 16.4 & 22.1 & 24.4 \\
\hline $\mathrm{B}_{2} \mathrm{O}_{3}$ & 5.0 & 5.0 & 5.0 & 5.0 & 5.0 \\
\hline $\mathrm{ZrO}_{2}$ & 7.0 & 7.0 & 7.0 & 7.0 & 7.0 \\
\hline $\mathrm{SiO}_{2}$ & 0.0 & 3.0 & 0.0 & 0.0 & 0.0 \\
\hline
\end{tabular}

To determine the total content of glass components in the simulant solution, the solution was calcined with an addition of silica. A $10-\mathrm{mL}$ solution sample was pipetted over $10 \mathrm{~g}$ of pure silica, dried at $105 \pm 5^{\circ} \mathrm{C}$ and slowly heated to $1050^{\circ} \mathrm{C}$. The mass of glass components in the simulant solution was 172 $\mathrm{g} / \mathrm{L}$. Based on this result, the saltcake solution was partly evaporated and spiked with Re (in the form of $\mathrm{Re}_{2} \mathrm{O}_{7}$ ) as a Tc surrogate at the level of 0.01 mass $\% \mathrm{ReO}_{2}$ in glass. Batches were prepared by mixing the saltcake with the soil, $\mathrm{H}_{3} \mathrm{BO}_{3}, \mathrm{ZrO}_{2}$, and $\mathrm{SiO}_{2}$, and sugar to reduce $\mathrm{Fe}_{2} \mathrm{O}_{3}$ to $\mathrm{FeO}$. Extra sugar was added to decompose $\mathrm{Na}_{8}\left(\mathrm{AlSiO}_{4}\right)_{6}\left(\mathrm{NO}_{2}\right)_{2}$, a compound that has a sodalite structure and persists to high temperatures (possibly up to $1450^{\circ} \mathrm{C}$ ), and to compensate for losses due to combustion. Batches were dried, calcined, and melted under argon atmosphere. The glass was poured on a stainless steel plate, crushed in the tungsten carbide mill, and remelted under argon. The same pouring procedure was followed as for the preliminary glasses.

Table 3.15. Compositions of ASCM and ARCM Glasses (in mass fractions)

\begin{tabular}{||l|c|c|c|c|c|c||}
\hline & ASCM-01 & ASCM-02 & ASCM-03 & ASCM-04 & ASCM-05 & ARCM-01 \\
\hline $\mathrm{Al}_{2} \mathrm{O}_{3}$ & 0.0989 & 0.0988 & 0.1031 & 0.0961 & 0.0933 & 0.1059 \\
\hline $\mathrm{B}_{2} \mathrm{O}_{3}$ & 0.0500 & 0.0500 & 0.0500 & 0.0500 & 0.0500 & 0.0501 \\
\hline $\mathrm{CaO}$ & 0.0375 & 0.0377 & 0.0394 & 0.0362 & 0.0350 & 0.0371 \\
\hline $\mathrm{Cl}$ & 0.0018 & 0.0015 & 0.0015 & 0.0020 & 0.0022 & 0.0017 \\
\hline $\mathrm{Cr}_{2} \mathrm{O}_{3}$ & 0.0009 & 0.0008 & 0.0008 & 0.0010 & 0.0011 & 0.0016 \\
\hline $\mathrm{F}$ & 0.0007 & 0.0006 & 0.0006 & 0.0008 & 0.0009 & 0.0004 \\
\hline $\mathrm{Fe}_{2} \mathrm{O}_{3}$ & 0.0633 & 0.0636 & 0.0665 & 0.0611 & 0.0590 & 0.0624 \\
\hline $\mathrm{K}_{2} \mathrm{O}$ & 0.0176 & 0.0176 & 0.0183 & 0.0171 & 0.0166 & 0.0172 \\
\hline $\mathrm{MgO}$ & 0.0097 & 0.0098 & 0.0102 & 0.0094 & 0.0091 & 0.0096 \\
\hline $\mathrm{Na}_{2} \mathrm{O}$ & 0.2000 & 0.1700 & 0.1700 & 0.2200 & 0.2400 & 0.2000 \\
\hline $\mathrm{P}_{2} \mathrm{O}_{5}$ & 0.0060 & 0.0053 & 0.0054 & 0.0064 & 0.0068 & 0.0061 \\
\hline $\mathrm{ReO}_{2}$ & 0.0001 & 0.0001 & 0.0001 & 0.0001 & 0.0001 & \\
\hline $\mathrm{SiO}_{2}$ & 0.4255 & 0.4575 & 0.4471 & 0.4111 & 0.3966 & 0.4199 \\
\hline $\mathrm{SO}_{3}$ & 0.0083 & 0.0069 & 0.0068 & 0.0093 & 0.0102 & 0.0084 \\
\hline \hline
\end{tabular}




\begin{tabular}{||l|l|l|l|l|l|l||}
\hline \hline $\mathrm{TiO}_{2}$ & 0.0097 & 0.0098 & 0.0102 & 0.0094 & 0.0091 & 0.0096 \\
\hline $\mathrm{ZrO}_{2}$ & 0.0700 & 0.0700 & 0.0700 & 0.0700 & 0.0700 & 0.0700 \\
\hline Total & 1.0000 & 1.0000 & 1.0000 & 1.0000 & 1.0000 & 1.0000 \\
\hline Soil & 0.6817 & 0.6852 & 0.7164 & 0.6586 & 0.6355 & 0.6725 \\
\hline Waste & 0.1983 & 0.1648 & 0.1636 & 0.2214 & 0.2445 & 0.2075 \\
\hline Additive & 0.1200 & 0.1500 & 0.1200 & 0.1200 & 0.1200 & 0.1200 \\
\hline
\end{tabular}

\subsubsection{Glass Composition and Summary of Properties}

Glasses were analyzed together with LRM-1 standard glass (Wolf et al. 1998) and bias-corrected following the methodology by Weier and Piepel (2003).

Table 3.16summarizes the results. Figure 3.11 compares measured and target concentrations as a function of $\mathrm{Na}_{2} \mathrm{O}$ concentration in glass for the components with a target mass fraction higher than 0.03. The measured concentrations were higher than the targets for $\mathrm{Na}_{2} \mathrm{O}$ and $\mathrm{SiO}_{2}$ and lower for $\mathrm{Al}_{2} \mathrm{O}_{3}, \mathrm{~B}_{2} \mathrm{O}_{3}$, $\mathrm{CaO}, \mathrm{Fe}_{2} \mathrm{O}_{3}$, and $\mathrm{ZrO}_{2}$. These differences were within roughly $15 \%$ of target concentrations except for $\mathrm{CaO}$ and $\mathrm{Fe}_{2} \mathrm{O}_{3}$, which showed a 30 to $50 \%$ difference. There was no trend in the measured-to-target ratio affected by the glass composition. The source for these differences could be a combined effect of analytical biases involved in soil, waste, and glass analyses as well as possible variation of soil compositions. The calculated retentions of $\mathrm{SO}_{3}$ in $\mathrm{ASCM}$ glasses ranged from 62 to $76 \%$.

The $\mathrm{Fe}(\mathrm{II}) / \mathrm{Fe}$ (total) fraction varied between 9 and $16 \%$ with a minimum at 22 mass $\% \mathrm{Na}_{2} \mathrm{O}$. ASCM glasses were prepared with sufficient sugar that can "theoretically" reduce most of the iron. However, as Table 3.17 shows, the glasses were oxidized either because of excessive sodalite formation or atmospheric oxygen that could consume the reducing agent to an unforeseen extent.

Table 3.16. Analyzed Compositions of ASCM and ARCM Glasses (in mass fractions) ${ }^{(a)}$

\begin{tabular}{||l|c|c|c|c|c|c||}
\hline & ASCM-01 & ASCM-02 & ASCM-03 & ASCM-04 & ASCM-05 & ARCM-01 \\
\hline $\mathrm{Al}_{2} \mathrm{O}_{3}$ & 0.0912 & 0.0915 & 0.0947 & 0.0872 & 0.0852 & 0.0939 \\
\hline $\mathrm{B}_{2} \mathrm{O}_{3}$ & 0.0465 & 0.0447 & 0.0454 & 0.0465 & 0.0474 & 0.0528 \\
\hline $\mathrm{CaO}$ & 0.0262 & 0.0266 & 0.0269 & 0.0250 & 0.0247 & 0.0348 \\
\hline $\mathrm{Cr}_{2} \mathrm{O}_{3}$ & 0.0010 & 0.0009 & 0.0009 & 0.0011 & 0.0012 & 0.0020 \\
\hline $\mathrm{Fe}_{2} \mathrm{O}_{3}$ & 0.0300 & 0.0306 & 0.0317 & 0.0286 & 0.0272 & 0.0288 \\
\hline $\mathrm{K}_{2} \mathrm{O}$ & 0.0125 & 0.0119 & 0.0119 & 0.0114 & 0.0118 & 0.0117 \\
\hline $\mathrm{MgO}$ & 0.0165 & 0.0164 & 0.0171 & 0.0156 & 0.0148 & 0.0118 \\
\hline $\mathrm{Na}_{2} \mathrm{O}$ & 0.2137 & 0.1793 & 0.1757 & 0.2297 & 0.2546 & 0.1722 \\
\hline $\mathrm{P}_{2} \mathrm{O}_{5}$ & 0.0049 & 0.0044 & 0.0043 & 0.0053 & 0.0058 & 0.0058 \\
\hline $\mathrm{ReO}_{2}$ & $1.55 \mathrm{E}-05$ & $1.58 \mathrm{E}-05$ & $1.43 \mathrm{E}-05$ & $1.50 \mathrm{E}-05$ & $3.70 \mathrm{E}-08$ & NA ${ }^{(\text {b) }}$ \\
\hline $\mathrm{SiO}_{2}$ & 0.4679 & 0.4969 & 0.4846 & 0.4433 & 0.4252 & 0.4216 \\
\hline $\mathrm{SO}_{3}$ & 0.0052 & 0.0044 & 0.0042 & 0.0058 & 0.0077 & NA ${ }^{(\text {b) }}$ \\
\hline $\mathrm{TiO}_{2}$ & 0.0092 & 0.0093 & 0.0100 & 0.0090 & 0.0085 & 0.0071 \\
\hline $\mathrm{ZrO}_{2}$ & 0.0609 & 0.0652 & 0.0623 & 0.0601 & 0.0609 & 0.0831 \\
\hline $\mathrm{Total}^{(\mathrm{a})} \mathrm{Average} \mathrm{from} \mathrm{duplicate} \mathrm{measurements.}_{(\text {b) }} \mathrm{ReO}_{2}$ and SO & 0.9859 & 0.9821 & 0.9697 & 0.9686 & 0.9753 & 0.9255 \\
\hline \hline
\end{tabular}




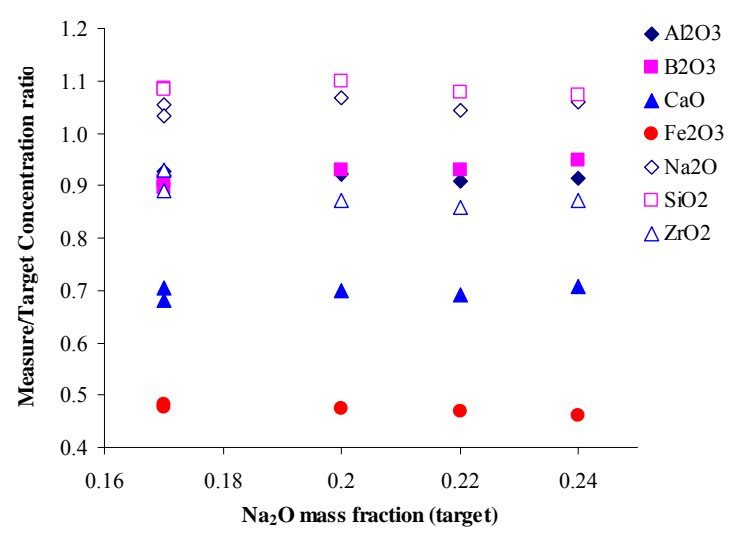

Figure 3.11. The Ratio of Measured to Target Concentrations for Selected Major Components in ASCM Glasses

Figure 3.12 and Figure 3.13 show that ASCM-01 and ARCM-01 are virtually identical despite minor differences in the compositions of the waste simulant and the actual waste. The larger differences between analytical compositions of ASCM-01 and ARCM-01 as compared to target compositions can be attributed to several possible sources of errors: 1) analyses of the wastes, 2) analyses of the glasses, 3) variation in soil composition, and 4) differences in the redox states of $\mathrm{Fe}$ (the $\mathrm{Fe}(\mathrm{II}) / \mathrm{Fe}$ fraction was measured only for ASCM-01).

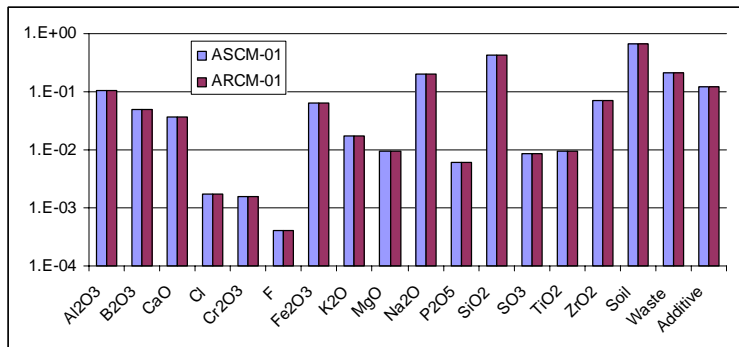

Figure 3.12. Targeted Mass Fractions of Components in Simulated (ASCM-01) and Radioactive (ARCM-01) Glasses

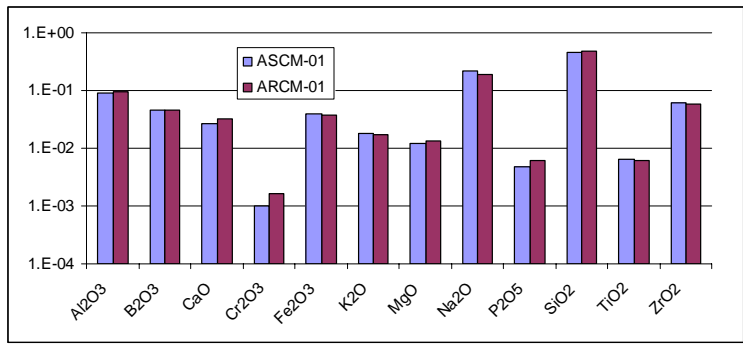

Figure 3.13. Analytical Mass Fractions of Components in Simulated (ASCM-01) and Radioactive (ARCM-01) Glasses

Table 3.17 summarizes the results of properties measured for ASCM glasses and the ARCM glass.

\subsubsection{Product Consistency Test}

Figure 3.14 shows the 7-day PCT normalized sodium releases from quenched and SC-treated samples of ASCM glasses as a function of $\mathrm{Na}_{2} \mathrm{O}$ concentration in glass. All ASCM glasses, even ASCM-05 with 24 mass $\% \mathrm{Na}_{2} \mathrm{O}$, passed the $2-\mathrm{g} / \mathrm{m}^{2}$ requirement. The data show that $\mathrm{SC}$ treatment slightly decreased the $r_{N a}$ for glasses with 20 mass $\% \mathrm{Na}_{2} \mathrm{O}$ or higher. There was no noticeable difference between ASCM- 02 and 
-03 , indicating that the effect of 3 mass $\% \mathrm{SiO}_{2}$ replacing soil had a negligible effect on PCT release. Predicted $r_{N a}$ values for quenched glasses are equal to or slightly lower than the measured releases for all ASCM glasses. Figure 3.15 and Figure 3.16 compare PCT $r_{i}$ values for Al, B, K, and Si with $r_{N a}$ values for quenched and SC-treated samples of ASCM glasses showing consistent results with the preliminary crucible test glasses discussed in Section 3.1.3. The Na concentration in the ASCM-01 leachate was 10\% higher than that from the ARCM-01 leachate regardless of whether the samples were quenched or subjected to the SC-treatment (Table 3.17, Figure 3.17, and Figure 3.18). The PCT solutions from ARCM-01 glasses were also analyzed for Tc and Re concentrations with ICP-MS. The results are summarized in Table 3.18 Re was not detected in all samples. These normalized Tc releases are several times lower than normalized $\mathrm{Na}$ or B releases given in Table 3.17. Since Na or B represent glass dissolution, the low Tc releases rule out a possibility of a selective leaching of Tc under PCT conditions.

Table 3.17. Summary of Properties for Simulant and Actual Waste Crucible Test Glasses

\begin{tabular}{|c|c|c|c|c|c|c|}
\hline Component & ASCM-01 & ASCM-02 & ASCM-03 & ASCM-04 & ASCM-05 & ARCM-01 \\
\hline \multicolumn{7}{|c|}{ 7-Day PCT Normalized Release $\left(\mathrm{g} / \mathrm{m}^{2}\right)$} \\
\hline$r_{N a}, \mathrm{Q}$ & 0.451 & 0.276 & 0.276 & 0.669 & 0.964 & 0.409 \\
\hline$r_{A l}, \mathrm{Q}$ & 0.119 & 0.082 & 0.084 & 0.153 & 0.196 & 0.125 \\
\hline$r_{B}, \mathrm{Q}$ & 0.220 & 0.139 & 0.146 & 0.301 & 0.417 & 0.221 \\
\hline$r_{C a}, \mathrm{Q}$ & 0.006 & 0.004 & 0.003 & 0.005 & 0.004 & 0.006 \\
\hline$r_{K}, \mathrm{Q}$ & 0.121 & 0.065 & 0.073 & 0.175 & 0.281 & 0.152 \\
\hline$r_{S i}, \mathrm{Q}$ & 0.137 & 0.101 & 0.101 & 0.169 & 0.214 & 0.160 \\
\hline$r_{\mathrm{Na}}, \mathrm{SC}$ & 0.406 & 0.280 & 0.266 & 0.612 & 0.858 & 0.371 \\
\hline$\overline{r_{A l}, \mathrm{SC}}$ & 0.113 & 0.078 & 0.080 & 0.141 & 0.177 & 0.123 \\
\hline$r_{B}, \mathrm{SC}$ & 0.206 & 0.129 & 0.135 & 0.278 & 0.381 & 0.155 \\
\hline$r_{C a}, \mathrm{SC}$ & 0.004 & 0.004 & 0.004 & 0.005 & 0.004 & 0.006 \\
\hline$r_{K}, \mathrm{SC}$ & 0.098 & 0.056 & 0.064 & 0.147 & 0.224 & 0.121 \\
\hline$r_{S i}, \mathrm{SC}$ & 0.132 & 0.096 & 0.098 & 0.163 & 0.204 & 0.154 \\
\hline \multicolumn{7}{|c|}{ 14-Day VHT Mass Loss $\left(g / \mathrm{m}^{2}\right)$} \\
\hline$m, \mathrm{Q}$ & 5.16 & 16.59 & 5.16 & 221.16 & 1389.03 & 0.00 \\
\hline$m, \mathrm{SC}$ & 13.11 & 4.00 & 3.96 & 9.15 & 935.44 & 11.69 \\
\hline \multicolumn{7}{|c|}{ TCLP Releases (concentration in $\mathbf{m g} / \mathbf{L})^{(\mathbf{a})}$} \\
\hline$c_{B}, \mathrm{Q}$ & 0.51 & 0.40 & 0.46 & 0.68 & 0.77 & 1.00 \\
\hline$c_{C r}, \mathrm{Q}$ & 0.022 & 0.015 & 0.011 & 0.021 & 0.015 & 0.007 \\
\hline$c_{B}, \mathrm{SC}$ & 0.60 & 0.52 & 0.86 & 0.47 & 0.92 & 1.20 \\
\hline$c_{C r}, \mathrm{SC}$ & ND & 0.005 & 0.033 & 0.015 & 0.059 & 0.030 \\
\hline \multicolumn{7}{|c|}{ Density $\left(\mathrm{g} / \mathrm{cm}^{3}\right)$} \\
\hline$\rho, \mathrm{Q}$ & 2.649 & 2.635 & 2.654 & 2.655 & 2.655 & 2.645 \\
\hline$\rho, \mathrm{SC}$ & 2.666 & 2.651 & 2.655 & 2.671 & 2.672 & 2.651 \\
\hline \multicolumn{7}{|c|}{ Iron Redox [Fe(II)/Fe(total)] } \\
\hline $\mathrm{Fe}(\mathrm{II}) / \mathrm{Fe}$ & 0.09 & 0.13 & 0.16 & 0.07 & 0.14 & \\
\hline $\begin{array}{ll}\text { (a) } & \text { The italici } \\
(0.25 \mathrm{mg} / \mathrm{l}\end{array}$ & $\begin{array}{l}\text { values in hig } \\
\text { Cr in all gla }\end{array}$ & $\begin{array}{l}\text { hted cells are } \\
; 0.5 \text { and } 1.2\end{array}$ & $\begin{array}{l}\text { timated result } \\
\mathrm{g} / \mathrm{L} \text { for } \mathrm{B} \text { in } \mathrm{A}\end{array}$ & $\begin{array}{l}\text { ecause they ar } \\
\mathrm{M} \text { glasses and }\end{array}$ & $\begin{array}{l}\text { elow the repor } \\
\text { RCM-01 glass }\end{array}$ & $\begin{array}{l}\text { limits } \\
\text { pectively). }\end{array}$ \\
\hline
\end{tabular}




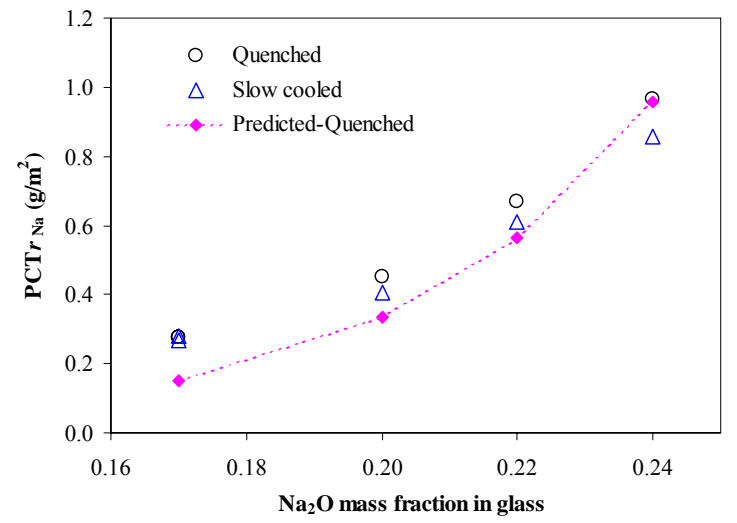

Figure 3.14. 7-Day

PCT $r_{N a}$ as a Function

of $\mathrm{Na}_{2} \mathrm{O}$ Concentration in ASCM Glasses

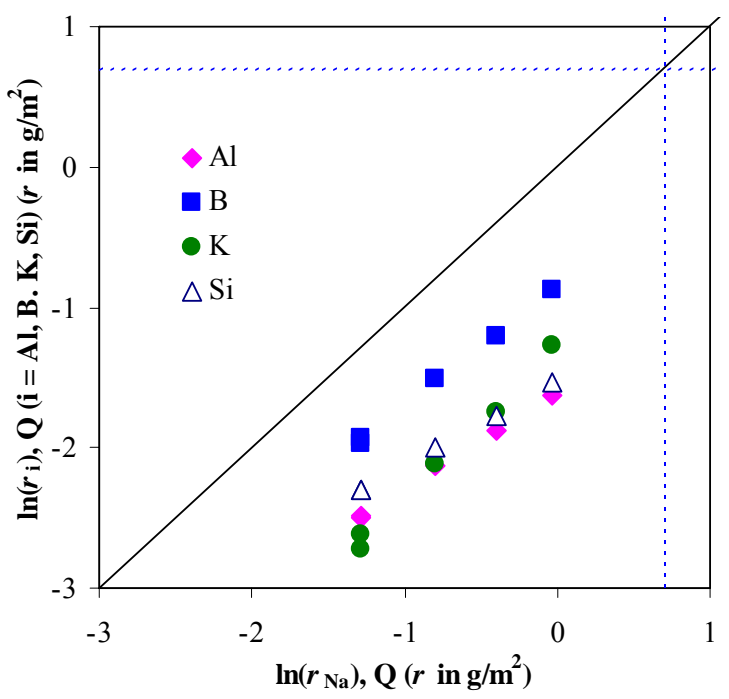

Figure 3.15.

Comparison of PCT

Elemental Releases of

Various Components

from Quenched ASCM

Glasses with $\mathrm{Na}$

Release (the dashed

line corresponds to

$$
r_{i}=2 \mathrm{~g} / \mathrm{m}^{2} \text { ) }
$$

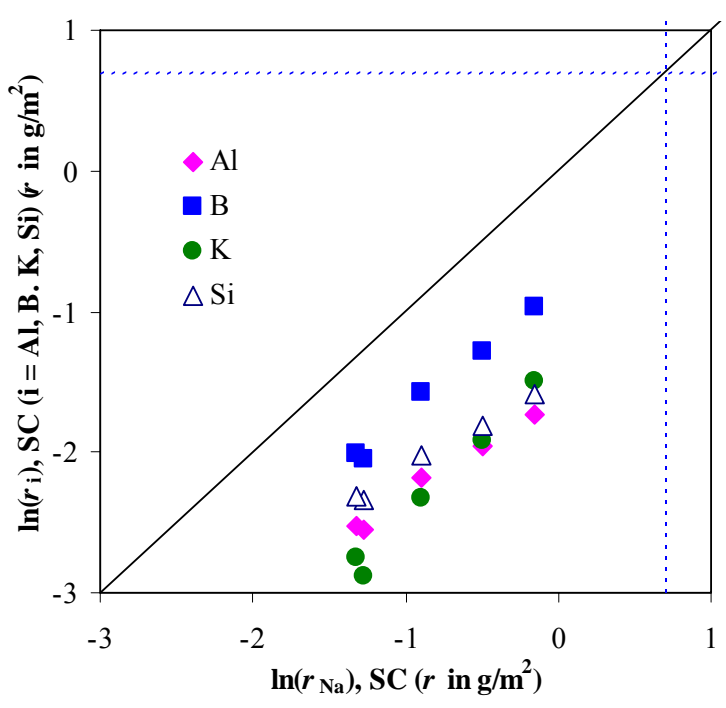

Figure 3.16.

Comparison of PCT

Elemental Releases of

Various Components

from SC Treated

ASCM Glasses with $\mathrm{Na}$

Release (the dashed

line corresponds to

$$
r_{i}=2 \mathrm{~g} / \mathrm{m}^{2} \text { ) }
$$




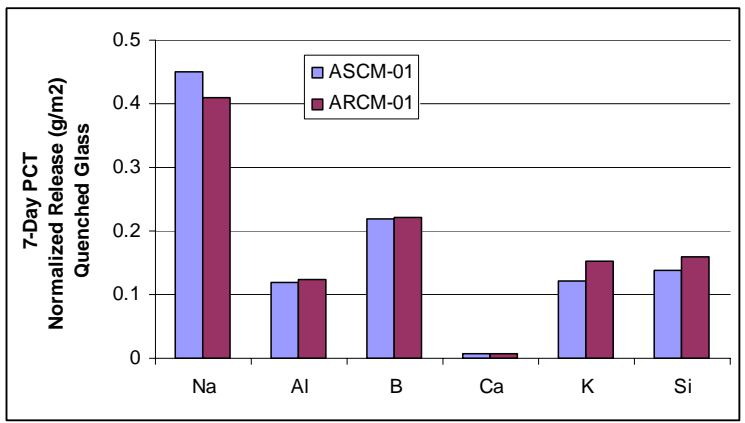

Figure 3.17. 7-Day PCT Normalized Releases from Simulated (ASCM-01) and Radioactive (ARCM-01) Quenched Glasses

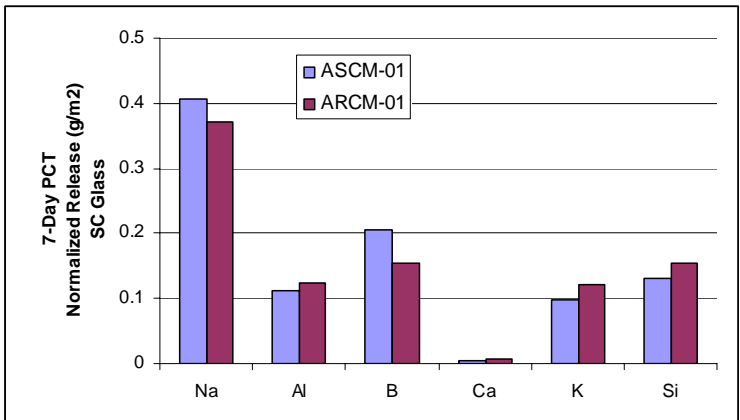

Figure 3.18. 7-Day PCT Normalized Releases from Simulated (ASCM-01) and Radioactive (ARCM-01) SC Glasses

Table 3.18. Concentration of Tc in PCT Solutions from ARCM-01 Glasses and Normalized Tc Releases

\begin{tabular}{||c|c|c||}
\hline Glass & $\boldsymbol{c}_{\mathbf{T c}}, \mathbf{m g} / \mathbf{L}$ & $\boldsymbol{r}_{\mathbf{T c}}, \mathbf{g} / \mathbf{m}^{2}$ \\
\hline ARCM-1-Q(1) & $2.96 \mathrm{E}-04$ & 0.054 \\
\hline ARCM-1-Q(2) & $2.94 \mathrm{E}-04$ & 0.054 \\
\hline ARCM-1-SC(1) & $2.06 \mathrm{E}-04$ & 0.038 \\
\hline ARCM-1-SC(2) & $2.15 \mathrm{E}-04$ & 0.039 \\
\hline
\end{tabular}

\subsubsection{Vapor Hydration Test and Toxicity Characteristic Leach Procedure}

Figure 3.19 shows 14-day VHT average corrosion rates from quenched and SC-treated samples of ASCM glasses as a function of $\mathrm{Na}_{2} \mathrm{O}$ concentration in glass. Except for ASCM- 05 with 24 mass $\% \mathrm{Na}_{2} \mathrm{O}, \mathrm{ASCM}$ glasses passed the $50 \mathrm{~g} /\left(\mathrm{m}^{2} \cdot \mathrm{d}\right)$ requirement (see the dashed line). The corrosion rate was decreased by SC treatment, at least for glasses with 22 mass $\% \mathrm{Na}_{2} \mathrm{O}$ or higher. For glasses with 20 mass $\% \mathrm{Na}_{2} \mathrm{O}$ or lower, the corrosion rate was too small to detect a difference.

Figure 3.20 shows the TCLP normalized B releases from quenched and SC-treated samples of ASCM glasses as a function of $\mathrm{Na}_{2} \mathrm{O}$ concentration in glass. Table 3.17 shows that the $\mathrm{Cr}$ releases in all the glasses, even for ASCM- 05 with 24 mass $\% \mathrm{Na}_{2} \mathrm{O}$, pass the UTS limit of the $0.6 \mathrm{mg} / \mathrm{L}$ requirement by at least an order of magnitude. As shown in Figure 3.20, normalized B releases were slightly increased after $\mathrm{SC}$ treatment except for one glass (ASCM-04). Strangely, $\mathrm{Na}_{2} \mathrm{O}$ content had little effect on TCLP B release, which is at variance with model prediction.

According to Table 3.17, no measurable corrosion was detected for ARCM-01 quenched glass and barely measurable corrosion for ARCM-01 SC. The differences in corrosion behavior between ASCM and ARCM glasses are within the limits of variations expected for repeated experiments with an identical composition. The TCLP B release was twice as high from ARCM-01 samples, both quenched and SCtreated, than from ASCM-01 samples. However, low B concentrations that are very close to or below reporting limits and extremely low $\mathrm{Cr}$ concentrations well below reporting limit do not allow reasonable comparison. 


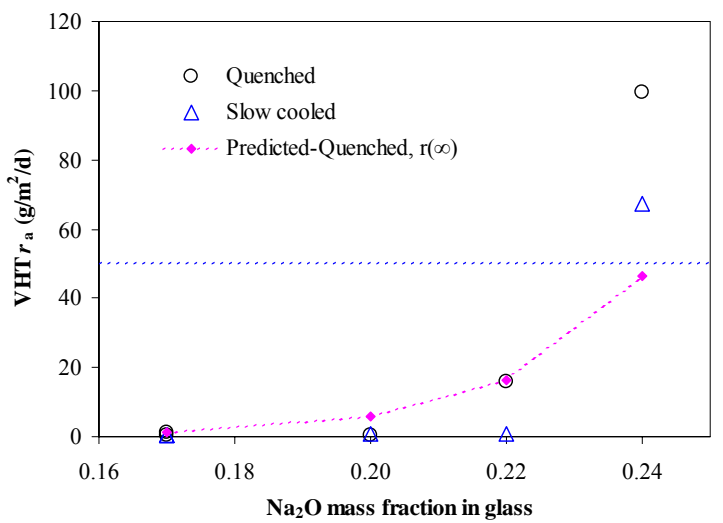

Figure 3.19. VHT $r_{a}$ as a Function of $\mathrm{Na}_{2} \mathrm{O}$ Concentration in ASCM Glasses

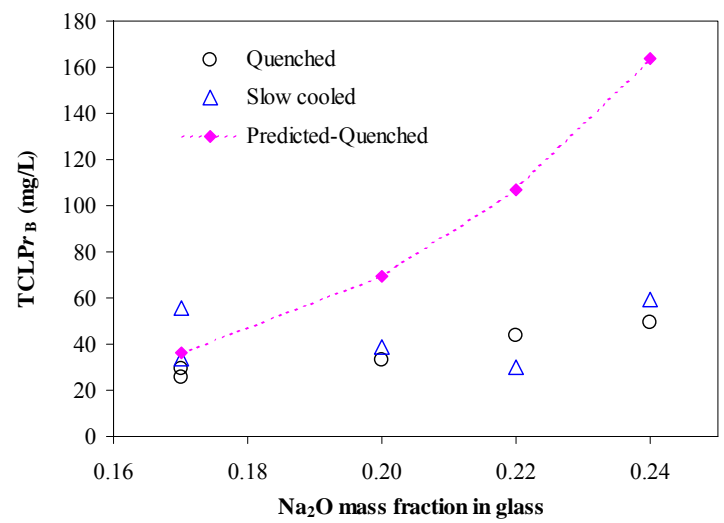

Figure 3.20. TCLP $\boldsymbol{r}_{B}$ as a Function of $\mathrm{Na}_{2} \mathrm{O}$ Concentration in ASCM Glasses

The crystalline alteration products were identified for a selected set of samples by XRD. Only ASCM-04 and -05 contained enough alteration products to allow the identification of the crystalline phases. These phases are listed in Table 3.13. It should be noted that the phases were identified from the crystal structures and not chemical analyses, so, other minerals or compositions with the structure of those identified may be present. For example, $\mathrm{SrZrO}_{3}$ is unlikely while $\mathrm{CaZrO}_{3}$ may have formed.

Table 3.19. Crystalline Alteration Product Summary

\begin{tabular}{l|l|c|l||}
\hline Sample ID & Time, $\mathrm{d}$ & Crystalline Phases $^{(\mathrm{a})}$ \\
\hline ASCM-04-Q & 14 & Analcime, Strontium Zirconium Oxide, Lithium Iron Oxide \\
\hline ASCM-05-Q & 14 & Sodalite, Lithium Iron Oxide \\
\hline
\end{tabular}

\subsubsection{Density}

As Figure 3.21 shows, the SC treatment increased the density as expected. The current model slightly overpredicted the density with a $2.0 \%$ difference on average. The densities of quenched and SC-treated ASCM-01 and ARCM-01 glasses are identical within $1 \%$.

\subsubsection{Secondary Phase identification}

Glasses with greater than 17 mass $\% \mathrm{Na}_{2} \mathrm{O}$ did not form any secondary phases during the $\mathrm{SC}$ heat treatment. However, in ASCM-02 and -03 glasses with 17 mass $\% \mathrm{Na}_{2} \mathrm{O}$, scattered spots or holes

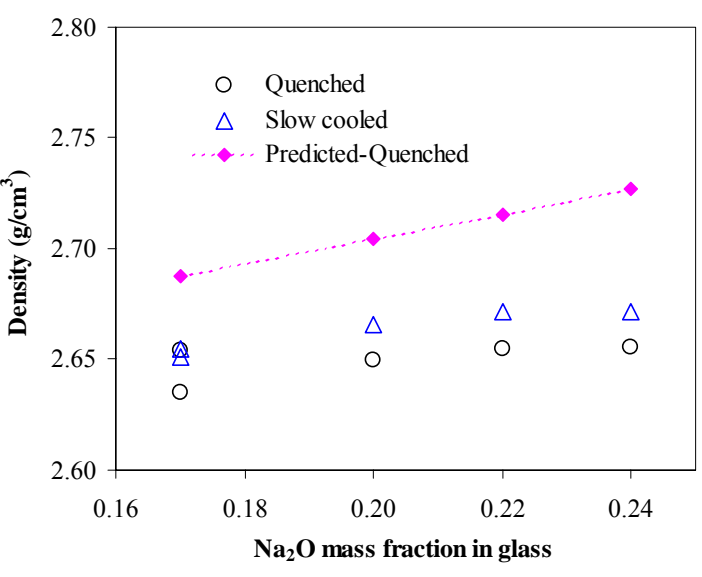

Figure 3.21. Density as a Function of $\mathrm{Na}_{2} \mathrm{O}$ Concentration in ASCM Glasses covered with deposits containing elemental $\mathrm{Fe}, \mathrm{Cr}$, and Ti were observed as shown in Figure 3.22 and Figure 3.23. Current test methods (PCT, VHT, and TCLP) do not show any impact of the presence of metallic inclusions and cavities on the product performance. 


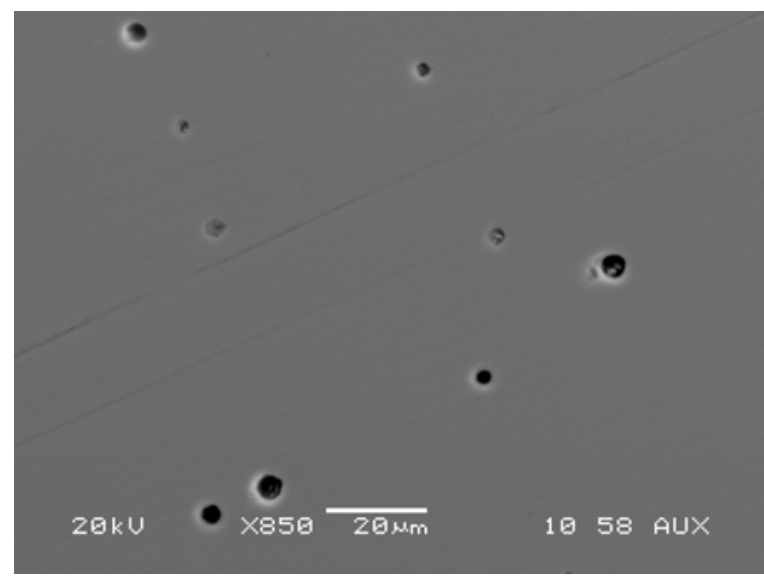

Figure 3.22. SEM Micrograph of Inclusions in SC-Treated ASCM-02 Glass

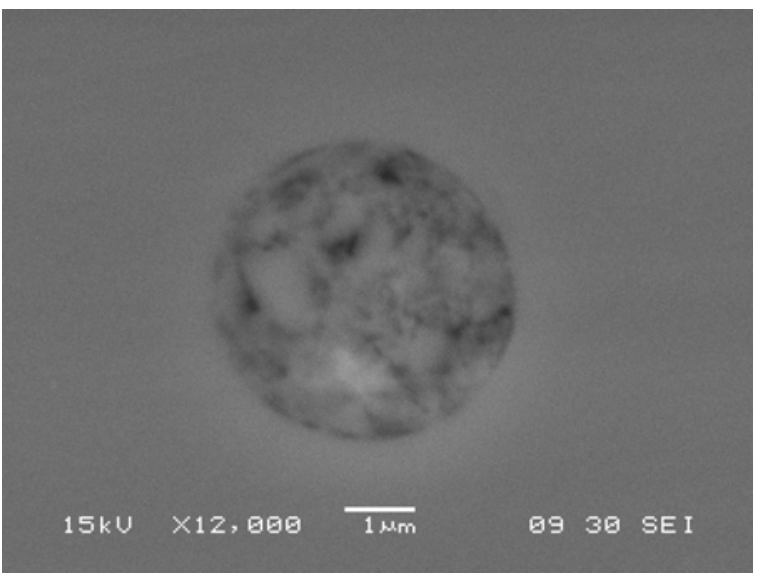

Figure 3.23. SEM Image of an Inclusion in SCTreated ASCM-02 Glass

\subsection{Engineering-Scale Simulant and Tc Tracer Tests}

\subsubsection{Summary}

An engineering-scale test (ES-1) with ASCM-01 composition and LAW simulant was performed to demonstrate ICV process feasibility with Hanford LAW simulant and to generate data related to product quality and off-gas. The chemical analyses of the glasses taken from various positions of the test-glass block indicated that the mixing of glass was very good. Multiple solid phases, including crystals and metallic droplets, were found in the engineering scale glass block. Dissolution of sand used to line the container walls as an insulating barrier increased the silica content in the glass. The glass performed (VHT, PCT, and TCLP testing) as well or better, due to a higher silica concentration, than comparable crucible melts and well below any imposed constraints. Another engineering-scale test (ES-2) was performed duplicating ES-1 with an addition of $\sim 1.46 \mathrm{mCi}$ of ${ }^{99} \mathrm{Tc}$ as a tracer. Like ES-1, the composition of the bulk glass from extreme points within the block had a nearly uniform composition.

\subsubsection{Feed Preparation}

The engineering-scale study was performed with ASCM01 glass. The content of glass components in the saltcake solution was measured and the saltcake volume was reduced as described in previous section. Of the two tests performed, the first (ES-1) used Re as a Tc surrogate, and the second (ES-2) used both Re and Tc. The received $\sim 40 \mathrm{~L}$ of saltcake solution was concentrated with evaporation using a heat lamp, flowing air, and a motorized agitator. Adding Re as a Tc surrogate for ES-1 proceeded as described for the crucible tests. Half of the solution was poured into a black iron pan placed in a water bath.

Table 3.20. Batch Materials for ES-1

\begin{tabular}{||l|r|r||}
\hline & \multicolumn{2}{|c||}{ Mass per batch (kg) } \\
\hline Dry batch amount & 39.64 & 34.01 \\
\hline Dry soil & 21.35 & 18.31 \\
\hline Saltcake (reduced volume) & 28.33 & 24.30 \\
\hline $\mathrm{H}_{3} \mathrm{BO}_{3}$ & 2.79 & 2.39 \\
\hline $\mathrm{ZrO}_{2}$ & 2.17 & 1.86 \\
\hline Total & 54.64 & 46.86 \\
\hline
\end{tabular}


The corresponding amounts of soil and $\mathrm{ZrO}_{2}$ (Table 3.20) in two batches were each mixed in a V-blender for 30 to $60 \mathrm{~min}$. The soil- $\mathrm{ZrO}_{2}$ mixture was added to the solution in the black iron pan. The paste was thoroughly homogenized with a rod while heating the pan with heat lamps and flowing compressed air. The dried batches were combined, mixed, and finally melted in an engineering-scale facility. An extra amount of flux materials was added to the top of the dried feed. Table 3.21 summarizes the amount of flux materials used in each ES test. The resulting ES-1 block of glass was removed from the melter to characterize for glass-composition distribution and glass properties (Figure 3.24).

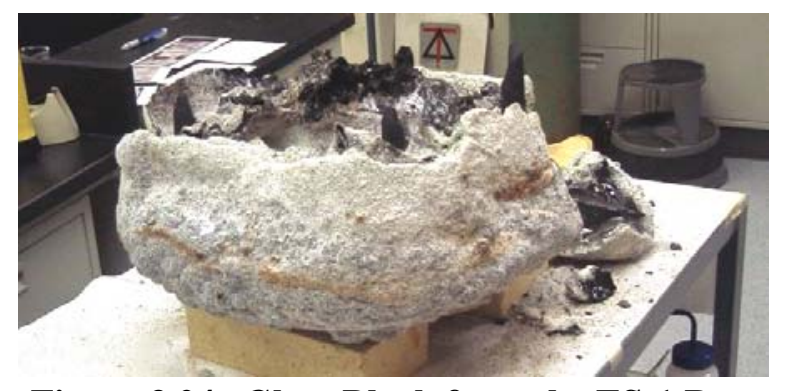

Figure 3.24. Glass Block from the ES-1 Run

The batch for ES-2 was made from the same masses of soil, $\mathrm{H}_{3} \mathrm{BO}_{3}$, and $\mathrm{ZrO}_{2}$ as in ES-1. Batch preparation and melting were also the same. The amount of ${ }^{99} \mathrm{Tc}$ (as $\mathrm{NH}_{4} \mathrm{TcO}_{4}$ solution) stirred into the saltcake was $1.46 \mathrm{mCi}\left(0.0885 \mathrm{~g}{ }^{99} \mathrm{Tc}\right)$.

Clusters of sand particle

s occurred in some areas, swept to the bulk glass from the sides and bottom by the convective currents in the melt. In some areas of the melt top and corners, sand particles collected as the less-dense sand grains floated to the uneven surface pockets at the top. Samples for chemical analysis

Table 3.21. Amount of Flux Materials Used in Each ES Test

\begin{tabular}{||c|c|c||}
\hline \hline & ES-1 & ES-2 \\
\hline Hanford soil & 5.995 & 5.624 \\
\hline $\mathrm{NaNO}_{3}$ & 4.110 & 3.855 \\
\hline $\mathrm{ZrO}_{2}$ & 0.598 & 0.561 \\
\hline $\mathrm{B}_{2} \mathrm{O}_{3}$ & 0.419 & 0.393 \\
\hline Total & 11.122 & 10.433 \\
\hline oxide mass & 8.511 & 7.983 \\
\hline
\end{tabular}
were taken several centimeters from the glass-sand interface to avoid including any sand particles. Figure 3.25 and Figure 3.26 show cross-sections of the glass-sand interface.

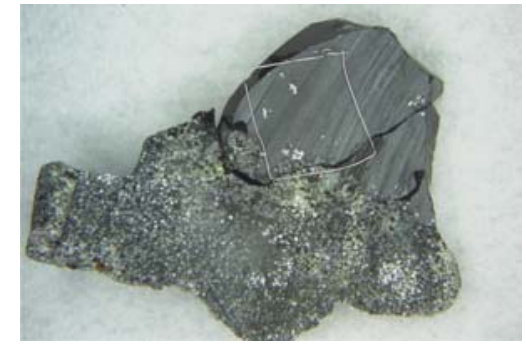

Figure 3.25. Glass-Sand Interface at Melt Bottom

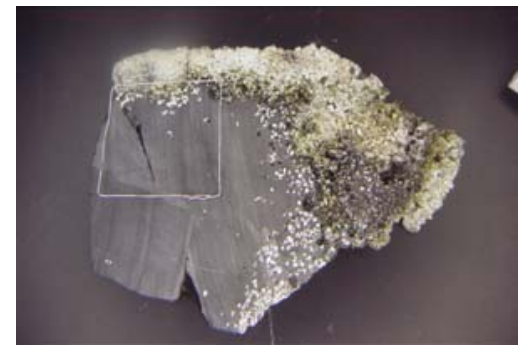

Figure 3.26. Glass-Sand Interface at Side-Wall and Top

\subsubsection{Chemical Analysis}

Table 3.22 summarizes the results of glass-composition and iron-redox analyses of five samples taken from different locations in the ES-1 glass block. 
Table 3.22. Analyzed Compositions (in mass fractions) and Redox of ES-1 Glasses

\begin{tabular}{||l|c|c|c|c|c|c||}
\hline & ES-1-1 & ES-1-2 & ES-1-3 & ES-1-4 & ES-1-5 & Average \\
\hline $\mathrm{Al}_{2} \mathrm{O}_{3}$ & 0.0719 & 0.0661 & 0.0700 & 0.0695 & 0.0712 & 0.0697 \\
\hline $\mathrm{B}_{2} \mathrm{O}_{3}$ & 0.0336 & 0.0332 & 0.0334 & 0.0341 & 0.0351 & 0.0339 \\
\hline $\mathrm{CaO}$ & 0.0205 & 0.0212 & 0.0216 & 0.0207 & 0.0218 & 0.0212 \\
\hline $\mathrm{Cr}_{2} \mathrm{O}_{3}$ & 0.0006 & 0.0006 & 0.0006 & 0.0006 & 0.0006 & 0.0006 \\
\hline $\mathrm{Fe}_{2} \mathrm{O}_{3}$ & 0.0208 & 0.0232 & 0.0201 & 0.0201 & 0.0206 & 0.0209 \\
\hline $\mathrm{K}_{2} \mathrm{O}$ & 0.0099 & 0.0113 & 0.0115 & 0.0102 & 0.0114 & 0.0109 \\
\hline $\mathrm{MgO}$ & 0.0121 & 0.0113 & 0.0114 & 0.0120 & 0.0114 & 0.0116 \\
\hline $\mathrm{Na}_{2} \mathrm{O}$ & 0.1499 & 0.1233 & 0.1500 & 0.1479 & 0.1503 & 0.1443 \\
\hline $\mathrm{P}_{2} \mathrm{O}_{5}$ & 0.0026 & 0.0022 & 0.0024 & 0.0024 & 0.0025 & 0.0024 \\
\hline $\mathrm{ReO}_{2}$ & 0.000012 & 0.000011 & 0.000011 & 0.000011 & 0.000011 & 0.000011 \\
\hline $\mathrm{SiO}_{2}$ & 0.6136 & 0.6602 & 0.6052 & 0.6127 & 0.6087 & 0.6201 \\
\hline $\mathrm{SO}_{3}$ & 0.0019 & 0.0017 & 0.0017 & 0.0015 & 0.0013 & 0.0016 \\
\hline $\mathrm{TiO}_{2}$ & 0.0072 & 0.0066 & 0.0066 & 0.0069 & 0.0066 & 0.0068 \\
\hline $\mathrm{ZrO}{ }_{2}$ & 0.0471 & 0.0515 & 0.0456 & 0.0467 & 0.0452 & 0.0472 \\
\hline $\mathrm{Total}^{2}$ & 0.9918 & 1.0124 & 0.9800 & 0.9854 & 0.9867 & 0.9913 \\
\hline \hline $\mathrm{Fe}(\mathrm{II}) / \mathrm{Fe}$ (total) & \multicolumn{1}{|c|}{1.00} & 1.00 & 0.87 & 1.00 & 0.90 & 0.95 \\
\hline \hline$(\mathrm{a})$ Fe(II)/Fe =1 suggests that a portion of Fe was reduced to Fe metal inclusions, which was actually observed. \\
\hline
\end{tabular}

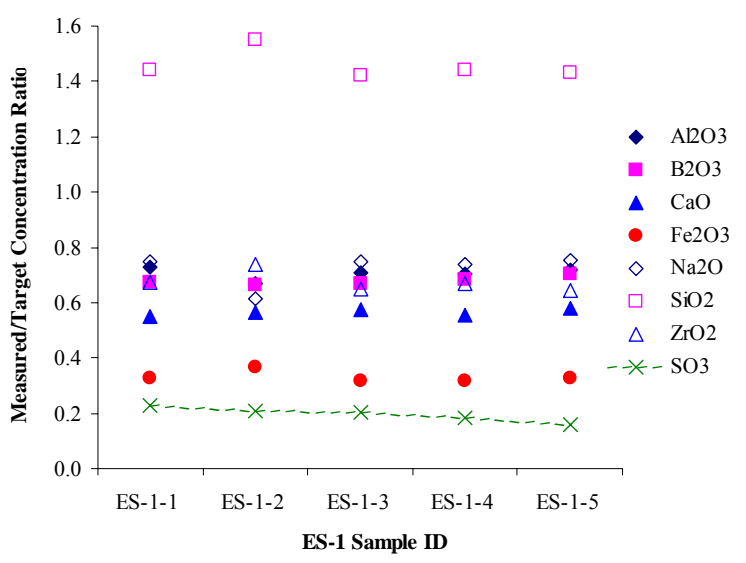

Figure 3.27. Ratio of Measured and Target Concentrations of $\mathrm{ReO}_{2}, \mathrm{SO}_{3}$, and Selected Major Components in ES-1 Glasses
Despite the color variations, the glass composition was reasonably constant, indicating good mixing by convection currents during the melting process. Figure 3.27 shows the comparison of measured and target concentrations of major glass components (those with a target mass fraction higher than 0.03) in ES-1 glass. As expected, measured concentrations were lower than the targets for all components except for $\mathrm{SiO}_{2}$. The relative difference of measured versus target concentrations ( 42 to $55 \%$ for $\mathrm{SiO}_{2}$ and 25 to $60 \%$ for the remaining major components) resulted from the dilution effect caused by the dissolution of silica sand that lined the container walls to insulate them from the glass melt (the ES test had a large melt surface area-to-volume ratio, $\sim 17 \mathrm{~m}^{-1}$; the mass fraction of sand dissolved in ES-1 glass estimated

from the $\mathrm{SiO}_{2}$ mass-balance ranges from 0.30 to 0.39 ). ES-1 glasses had a lower $\mathrm{SO}_{3}$ retention than the simulant crucible tests - the $\mathrm{SO}_{3}$ retention in glass ranged from 16 to $23 \%$ (relative). The iron-redox results listed in Table 3.22 indicate that the glass was highly reduced.

\subsubsection{Product Consistency Test}

A schematic of the ES-1 glass block in Figure 3.28 shows positions of glass samples taken for PCT, VHT, TCLP, and density. Figure 3.29 illustrates how samples ES-1 through -8 were taken from the side-wall area of the ES-1 glass block. The PCT sample came from close to the glass-sand interface. 
The normalized releases from ES- 1 glasses were roughly 1/3 lower than from ASCM-01 glass (compare Table 3.17 and Table 3.23). This is attributable to the dissolution of silica sand in ES-1. There was no noticeable difference between samples taken at different locations.

Table 3.23. PCT Normalized release $\left(\mathrm{g} / \mathrm{m}^{2}\right)$ for ES-1 Glasses

\begin{tabular}{||c|c|c|c|c|c||}
\hline \hline Component & ES-1-6 & ES-1-7 & ES-1-8 & ES-1-9 & ES-1-10 \\
\hline$r_{N a}$ & 0.152 & 0.140 & 0.150 & 0.132 & 0.143 \\
\hline$r_{A l}$ & 0.047 & 0.047 & 0.046 & 0.039 & 0.046 \\
\hline$r_{B}$ & 0.073 & 0.071 & 0.073 & 0.063 & 0.073 \\
\hline$r_{S i}$ & 0.126 & 0.120 & 0.129 & 0.126 & 0.124 \\
\hline $\begin{array}{l}6 \text { center of the glass block } \\
9 \text { between electrodes \#3 and \#4 }\end{array}$ & $\begin{array}{l}7 \text { bottom of the glass block } \\
10 \text { next to the electrode \#3 }\end{array}$ \\
\hline
\end{tabular}

\subsubsection{Vapor Hydration Test and Toxicity Characteristic Leaching Procedure}

Table 3.24 summarizes the 14-day VHT responses of the glass samples taken from different positions of the ES-1 glass block. Several samples were taken to span the sand layer-glass interface, i.e., sand soaked with glass, the interface, and the bulk glass outside the interface (Figure 3.30). The samples showed no visible corrosion after $200^{\circ} \mathrm{C}$ for 14 days. Only the sample taken from the area close to the graphite electrode (ES-1-10) and samples with sand from the bottom (ES-1-7S and 7M) showed signs of corrosion after the 14-day VHT.

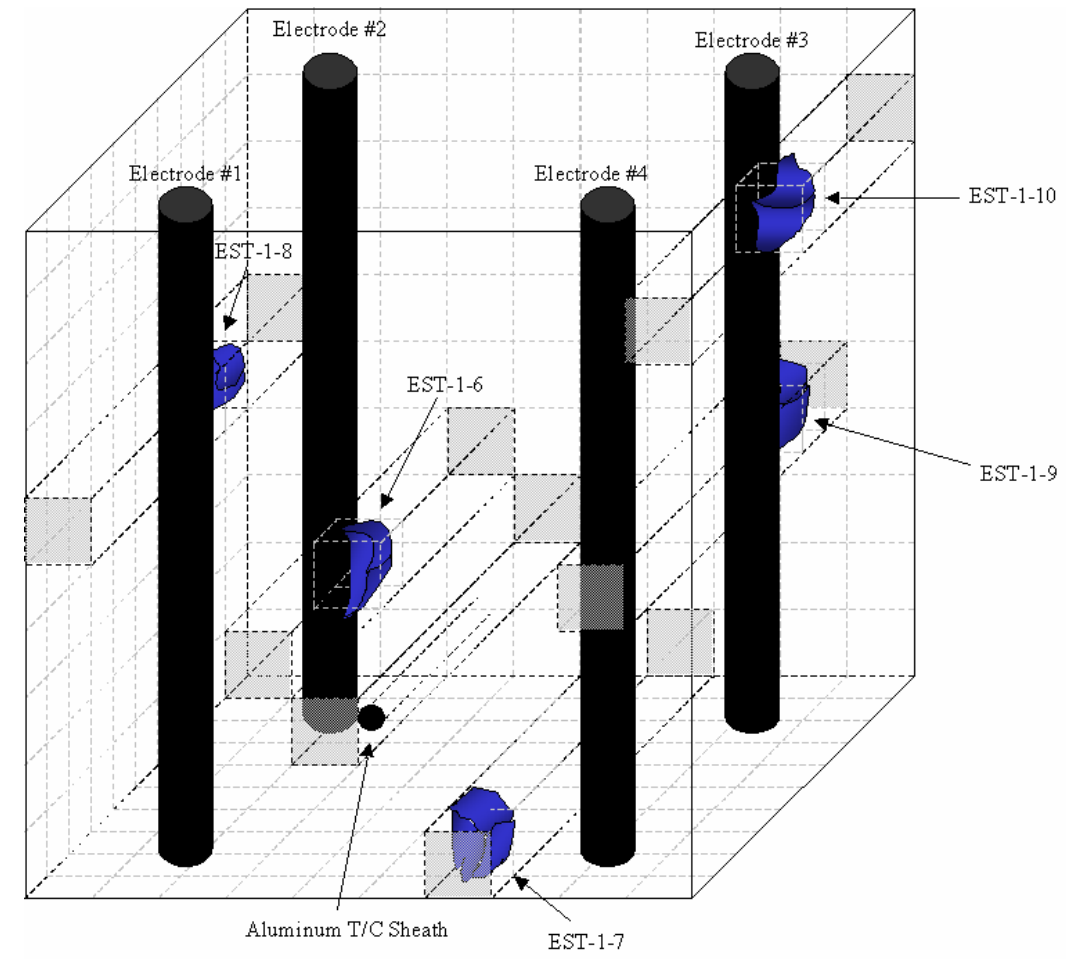

Figure 3.28. ES-1 Glass Block Showing the Areas from Where PCT, VHT, TCLP and Density Samples Were Obtained ${ }^{(a)}$

\footnotetext{
a The term "EST" used within the diagram is the same as "ES".
} 


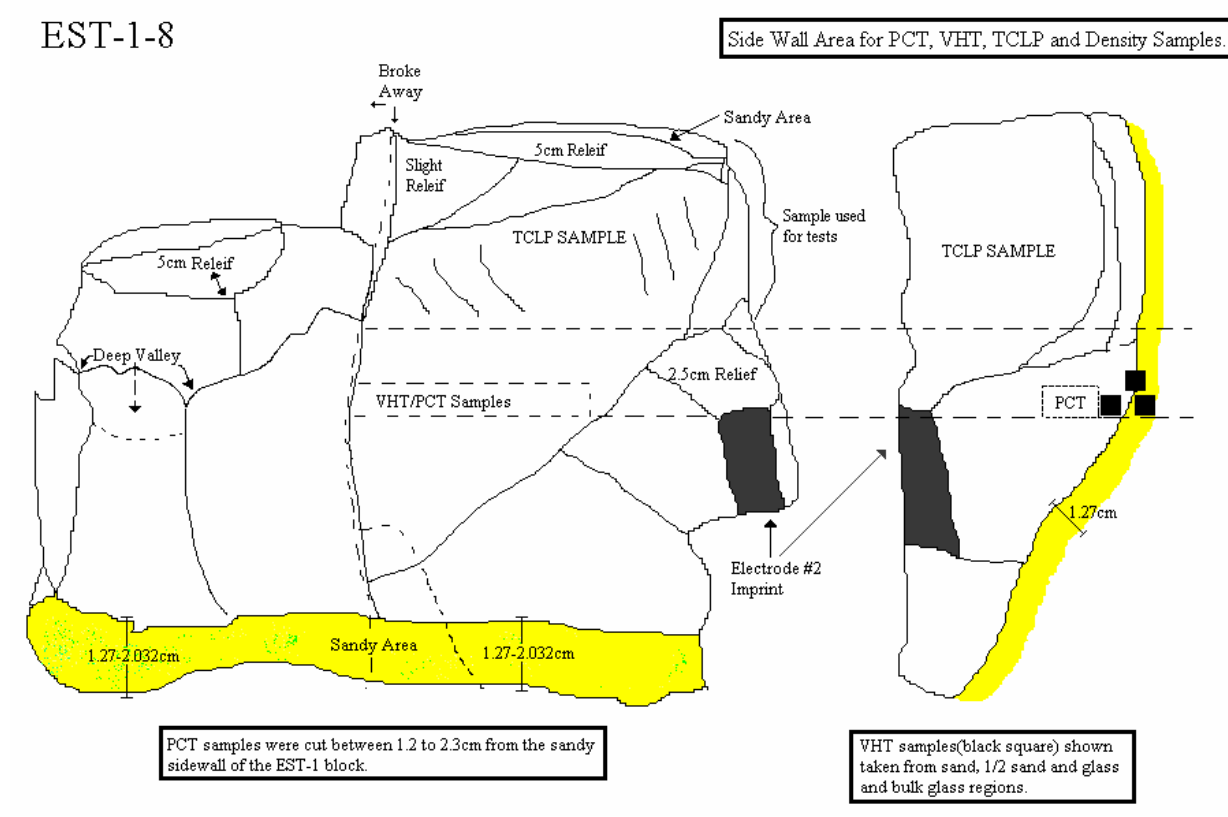

Figure 3.29. Illustration of How the ES-1-8 Samples for Glass Characterization Were Obtained from the Side-Wall Area of the ES-1 Glass Block ${ }^{(a)}$

Table 3.24. 14-day VHT Responses for ES-1 Glasses

\begin{tabular}{||c|c|c|c|c|c|c|c|c|c||}
\hline \hline Sample & ES-1-6 & ES-1-7S & ES-1-7M & ES-1-7G & ES-1-8S & ES-1-8M & ES-1-8G & ES-1-9 & ES-1-10 \\
\hline$m\left(\mathrm{~g} / \mathrm{m}^{2}\right)$ & 5.06 & 5.27 & 3.58 & 3.74 & 5.08 & 4.91 & 3.79 & 1.27 & 66.20 \\
\hline$r_{a}\left(\mathrm{~g} / \mathrm{m}^{2} / \mathrm{d}\right)$ & 0.36 & 0.38 & 0.26 & 0.27 & 0.36 & 0.35 & 0.27 & 0.09 & 4.68 \\
\hline 6 & 6 center of the glass block \\
9 between electrodes \#3 and \#4 bottom of the glass block & \multicolumn{1}{c}{ 10 next to the electrode \#3 mix of glassy and sand areas } \\
G glassy area \\
\hline
\end{tabular}
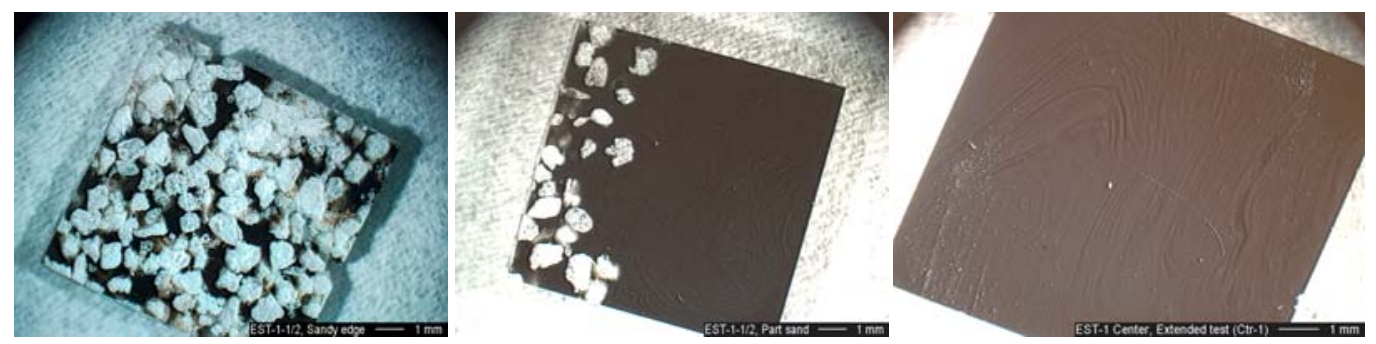

Figure 3.30. Optical Micrographs of VHT Coupons from Sand-Glass Interface Area, ES-1-8

Needle-like crystals were visible by microscope on the 14-day VHT ES-1-7S sample surface (Figure 3.31, left). Numerous small cavities were inside the sample. Reaction products are clearly visible Figure 3.31, right, on the 14-day VHT coupons from the sand-glass interface area (ES-1-7M).

(a) The term "EST" used within the diagram is the same as "ES." 


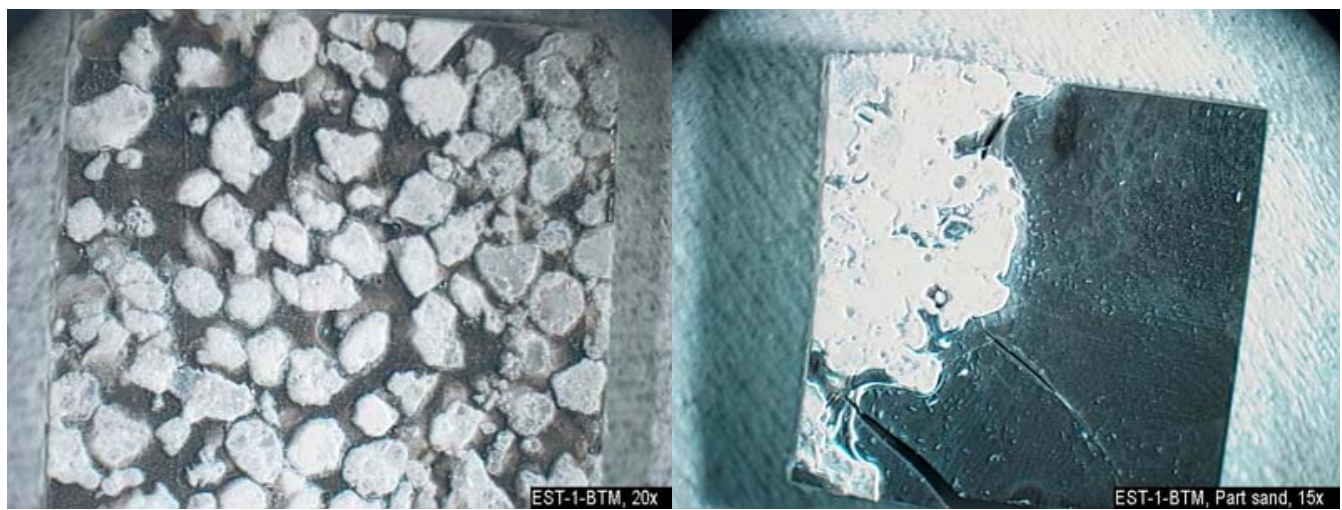

Figure 3.31. Optical Micrographs of VHT Coupons from Sandy Area (ES-1-7S, left) and SandGlass Interface Area (ES-1-7M, right) after 14 days at $200^{\circ} \mathrm{C}$ (coupon size is $10 \mathrm{~mm}$ )

Table 3.25 shows that the TCLP Cr releases are at least an order of magnitude lower than the UTS limit of $0.6 \mathrm{mg} / \mathrm{L}$.

Table 3.25. TCLP Responses for ES-1 Glasses

\begin{tabular}{||l|c|c|c|c|c|c||}
\hline Glass Sample & ES-1-6 & ES-1-7 & ES-1-8 & ES-1-9 & ES-1-10 & ES-1-Foam \\
\hline$C_{B}(\mathrm{mg} / \mathrm{L})$ & 0.23 & 0.36 & 0.33 & 0.2 & 0.24 & 1.1 \\
\hline$C_{C r}(\mathrm{mg} / \mathrm{L})$ & 0.0052 & $\mathrm{ND}$ & $\mathrm{ND}$ & 0.01 & $\mathrm{ND}$ & 0.009 \\
\hline$r_{B}(\mathrm{mg} / \mathrm{L})$ & 14.81 & 23.18 & 21.25 & 12.88 & 15.46 & 70.84 \\
\hline
\end{tabular}

\subsubsection{Density}

Density values (Table 3.26) span the range of $2.566 \pm 0.006 \mathrm{~g} / \mathrm{mL}$, which is lower than that of ASCM-01 glass because of the increased silica concentrations in ES-1 glass.

Table 3.26. Density of ES-1 Glass Samples $(\mathrm{g} / \mathrm{mL})$

\begin{tabular}{|c|c|c|c|c|c|c|c|}
\hline Sample & ES-1-6 & ES-1-7 & ES-1-8 & ES-1-9 & ES-1-10 & Mean & ASCM-01 \\
\hline$\rho$ & 2.567 & 2.565 & 2.564 & 2.572 & 2.561 & 2.566 & $2.649-2.666$ \\
\hline $\begin{array}{l}6 \text { center of th } \\
9 \text { between el }\end{array}$ & $\begin{array}{l}\text { lass bloc } \\
\text { rodes \#3 }\end{array}$ & & $\begin{array}{l}\text { ttom of } \\
\text { ext to th }\end{array}$ & $\begin{array}{l}\text { glass b } \\
\text { lectrod }\end{array}$ & & een e & des \#1 and \#2 \\
\hline
\end{tabular}

\subsubsection{Secondary-Phase Identification}

Four types of secondary-phases may occur in the ICV glass: oxide crystals, metals, gas bubbles, and inorganic salts. All these inclusions were observed in crucible melts as discussed in previous sections. A view of the ES-1 block clearly contains a multi-phased material, but most of the block mass is singlephased glass. No inclusions were found in the glass at the block exterior. Metallic (mainly $\mathrm{Fe}$ ) droplets were found near the electrodes, mostly on the electrode surface and at the bottom of the melt. The size of the droplets varied from $1 \mu \mathrm{m}$ to $2 \mathrm{~mm}$. Some droplets contained inclusions of $\mathrm{Cr}$, Ti, and $\mathrm{Zr}$. Most droplets were spherical in shape. Droplets of irregular shape are shown in Figure 3.33 and Figure 3.34. 
These large droplets $(\sim 0.5 \mathrm{~mm})$ consist of metallic Fe and are often surrounded by irregular metallic precipitates that are rich in $\mathrm{Fe}$ and $\mathrm{Cr}$. Note irregular inclusions of Ti.

The distribution of crystallinity within the transition layer between the glass and silica-sand lining was determined by XRD — see Figure 3.35. The transition begins with roughly 50 mass $\%$ of an amorphous phase and develops gradually to a fully amorphous material over roughly $4 \mathrm{~cm}$ in both sample locations.

\subsubsection{Sample Position and Identification for ES-2 Glass Composition Analyses}

After the melt of ES-2, the glass block was broken into several big pieces, from which five samples were selected for analysis by ICP-AES, and Re and Tc by ICP-MS (ES-2-1 and ES-2-5 from the center area on the melt, ES-2-3 and ES-2-4 from the electrode areas, and ES-2-2 from a section of the crust.). Insulating sand had been pulled into the melt and in some areas mixed through the glass.

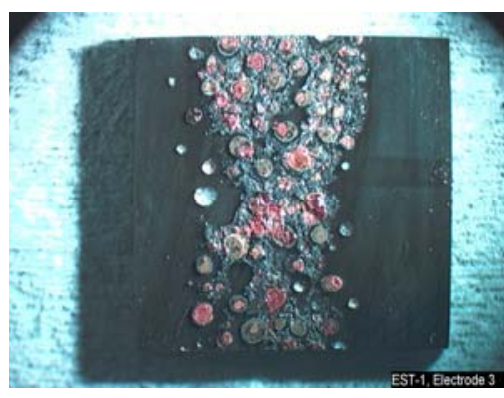

Figure 3.32. VHT Coupon from Glass near Electrode 3 (ES-1-10) with Metallic Droplets at the Contact with Electrode (iron was oxidized during the 14-days exposure to steam at $200^{\circ} \mathrm{C}$ )

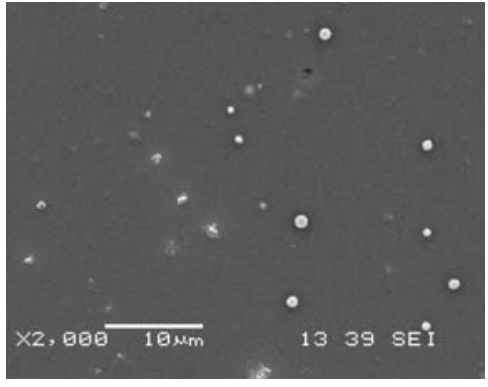

Figure 3.33. A Typical Section of Glass with Metallic Droplets (SEM micrograph)

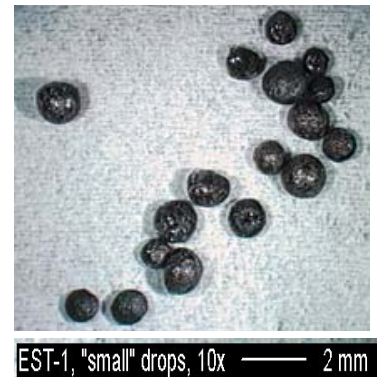

Figure 3.34. Metallic Droplets Released from ES-1 Glass Block During Sampling

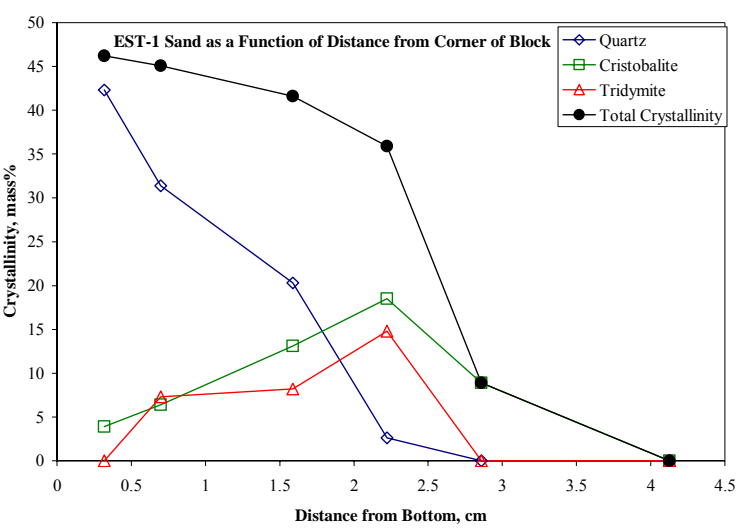

(a) Bottom Corner of the Block

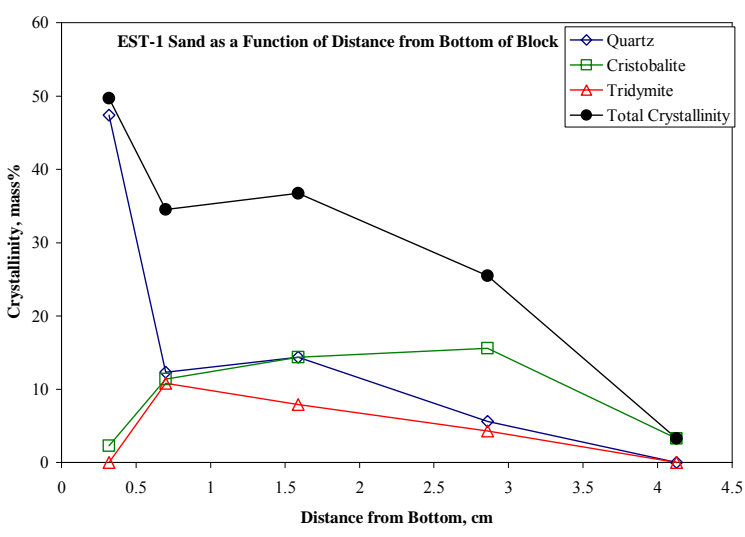

(b) Bottom Surface of the Block

Figure 3.35. $\mathrm{SiO}_{2}$ Concentration as a Function of Distance from the Surface of the ES-1 Block 


\subsubsection{Glass Composition}

Table 3.27 summarizes the results of glass-composition analyses of samples from different locations of the ES-2 glass block. Figure 3.36 compares measured and target concentrations of components with a target mass fraction higher than 0.03 . The effect of sand dissolution during the ES-2 process is evident. The extent of sand dissolution in ES-2 ranged from 0.18 to 0.23 , which was slightly less than in ES-1.

Table 3.27. Analyzed Compositions (in mass fraction) of ES-2 Glasses

\begin{tabular}{|c|c|c|c|c|c|c|}
\hline Component & ES-2-1 & ES-2-2 & ES-2-3 & ES-2-4 & ES-2-5 & Average \\
\hline $\mathrm{Al}_{2} \mathrm{O}_{3}$ & 0.0818 & 0.0853 & 0.0834 & 0.0763 & 0.0823 & 0.0818 \\
\hline $\mathrm{B}_{2} \mathrm{O}_{3}$ & 0.0442 & 0.0477 & 0.0451 & 0.0397 & 0.0442 & 0.0442 \\
\hline $\mathrm{CaO}$ & 0.0322 & 0.0337 & 0.0321 & 0.0298 & 0.0320 & 0.0320 \\
\hline $\mathrm{Cr}_{2} \mathrm{O}_{3}$ & 0.0007 & 0.0008 & 0.0007 & 0.0006 & 0.0007 & 0.0007 \\
\hline $\mathrm{Fe}_{2} \mathrm{O}_{3}$ & 0.0257 & 0.0303 & 0.0267 & 0.0208 & 0.0256 & 0.0258 \\
\hline $\mathrm{K}_{2} \mathrm{O}$ & 0.0117 & 0.0125 & 0.0117 & 0.0109 & 0.0109 & 0.0115 \\
\hline $\mathrm{MgO}$ & 0.0114 & 0.0120 & 0.0115 & 0.0106 & 0.0114 & 0.0114 \\
\hline $\mathrm{Na}_{2} \mathrm{O}$ & 0.1336 & 0.1448 & 0.1411 & 0.1261 & 0.1349 & 0.1361 \\
\hline $\mathrm{P}_{2} \mathrm{O}_{5}$ & 0.0035 & 0.0046 & 0.0040 & 0.0028 & 0.0037 & 0.0037 \\
\hline $\mathrm{ReO}_{2}$ & $9.73 \mathrm{E}-06$ & $5.55 \mathrm{E}-05$ & $1.66 \mathrm{E}-05$ & $8.98 \mathrm{E}-06$ & $1.01 \mathrm{E}-05$ & $2.02 \mathrm{E}-05$ \\
\hline $\mathrm{SiO}_{2}$ & 0.4920 & 0.4570 & 0.4967 & 0.5147 & 0.5052 & 0.4931 \\
\hline $\mathrm{SO}_{3}$ & $\mathrm{NA}^{(\mathrm{a})}$ & $\mathrm{NA}^{(\mathrm{a})}$ & $\mathrm{NA}^{(\mathrm{a})}$ & $\mathrm{NA}^{(\mathrm{a})}$ & $\mathrm{NA}^{(\mathrm{a})}$ & \\
\hline $\mathrm{TiO}_{2}$ & 0.0069 & 0.0073 & 0.0070 & 0.0064 & 0.0069 & 0.0069 \\
\hline $\mathrm{ZrO}_{2}$ & 0.0723 & 0.0771 & 0.0752 & 0.0603 & 0.0752 & 0.0720 \\
\hline Total & 0.9161 & 0.9131 & 0.9352 & 0.8990 & 0.9329 & 0.9193 \\
\hline${ }^{99} \mathrm{Tc}(\mu \mathrm{Ci} / \mathrm{g})$ & 0.0142 & 0.0305 & 0.0149 & 0.0103 & 0.0142 & 0.0168 \\
\hline
\end{tabular}

(a) Not analyzed - the analytical instruments used for these radioactive glasses were not capable of detecting sulfur.

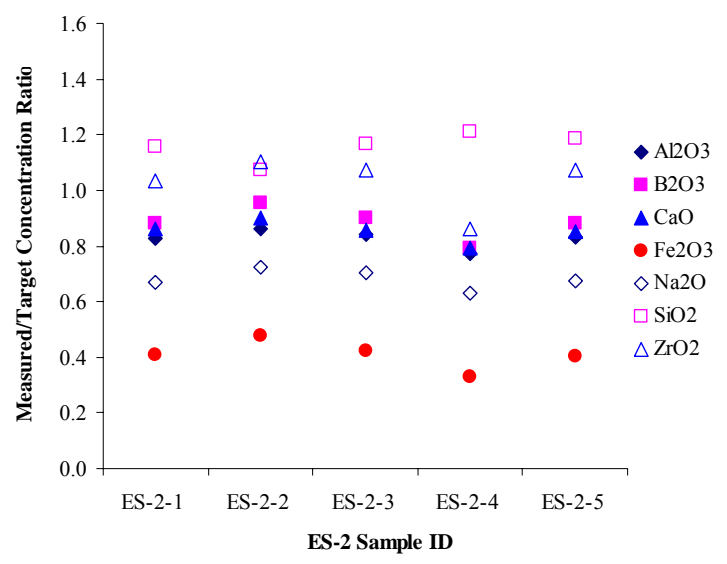

Figure 3.36. Ratio of Measured and Target Concentrations of ES-2 Glasses Components

As both the ES-1 and ES-2 melts progressed, large bubbles (up to $1 \mathrm{~L}$ in volume) formed around the electrodes where the off-gas from the melt was escaping. Bubbles collapsed and formed a mass of irregular glass lobes above the insulating sand layer over the melt surface. White powdery condensed volatiles were found on inner surfaces of the bubbles; some inner-bubble surfaces appeared reddish to metallic. Figure 3.37 shows SEM micrographs of the porous condensate materials from an ES-1 sample. Similar condensate from ES-2 was dissolved in DIW. Assuming that nearly all of the condensate was 
dissolved, only $0.06 \%$ of the Tc and $1.12 \%$ of the Re was in the condensate. For the Tc mass balance, the entire ES-2 glass block was ground to $\leq 1.5 \mathrm{~mm}$ size and well blended. Sample analysis showed that $93 \%$ Tc and $79 \%$ Re were retained in the waste form.
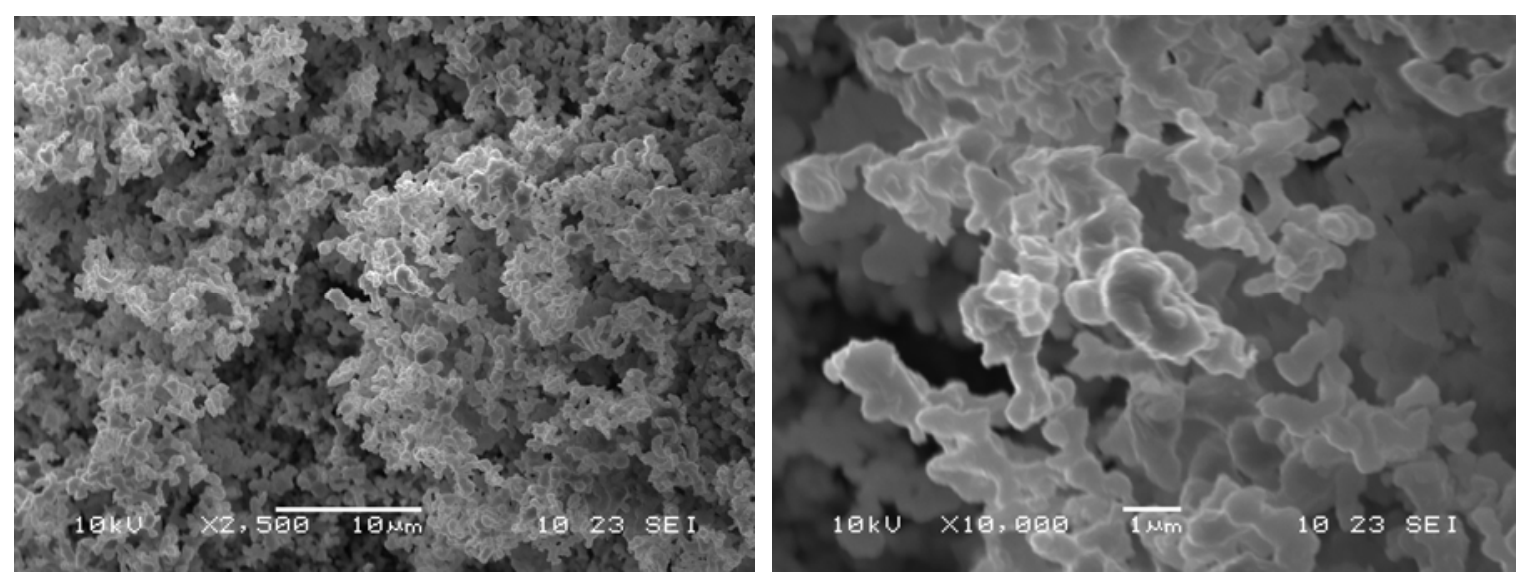

Figure 3.37. SEM Micrographs of White Condensate on the Inside of Large Glass Bubbles from ES-1 Melter Test (similar to the condensate from ES-2 that was dissolved in DIW for analyses)

\subsection{Initial Testing Summary and Conclusions}

The initial study to formulate and test simulated and actual Hanford LAW glasses to be produced by the ICV process was performed in five phases as described below.

1. A preliminary study identified a baseline glass that met the processing, product quality, and economic constraints of the ICV process applied to Hanford LAW. Sixteen glasses were formulated, fabricated, and tested. The key variables were waste loading and additive composition. Based on VHT and PCT responses and crystallinity in both quenched and slow cooled samples, the AMBG-13 was adopted as the baseline for scale-up and radioactive demonstrations. The AMBG-13 has 20 mass $\% \mathrm{Na}_{2} \mathrm{O}$, its viscosity is $10 \mathrm{~Pa} \cdot \mathrm{s}$ at $1238^{\circ} \mathrm{C}$, and its electrical conductivity at $1238^{\circ} \mathrm{C}$ is $39.7 \mathrm{~S} / \mathrm{m}$. In a limited series of tests, the oxygen fugacity of ASCM-04 glass was varied to determine its impact on the VHT response. The VHT response improved as the glass became more reduced.

2. Crucible tests were performed with the LAW simulant using five glasses with varied waste loading ranging. All the glasses passed the PCT and TCLP requirements with a large margin, and the VHT requirement was met in four glasses (the glass with the highest $\mathrm{Na}_{2} \mathrm{O}$ concentration of 24 mass $\%$ failed the test).

3. The radioactive glass (ARCM-01) with almost the same target composition as AMBG-13 (from preliminary tests) and ASCM-01 (from simulant crucible melts) was prepared with the actual Hanford LAW. No noticeable difference in measured properties was observed between these two glasses. The normalized Tc release was several times lower than normalized $\mathrm{Na}$ or $\mathrm{B}$ release, suggesting that there was no selective leaching of Tc under the PCT condition. 
4. An engineering-scale test with LAW simulant produced a glass block with nearly uniform composition. The glass performed better than comparable crucible melts and well below any imposed constraints. A significant dissolution of sand used to line the container walls as an insulating barrier improved the glass responses under PCT, VHT and TCLP conditions as compared to crucible melts.

5. An engineering-scale test with the LAW simulant as $\sim 1.46 \mathrm{mCi}$ of ${ }^{99} \mathrm{Tc}$ as a tracer produced a glass-block with composition similar to that of the nonradioactive simulant. 


\subsection{First AMEC Large-Scale Glass Test (LS-1)}

\subsection{Introduction}

This test has been designed to provide information necessary for the assessment of the ICV process and product quality performance and to support subsequent large-scale tests as well as full-scale engineering design. Glass formulation for the large-scale (LS) testing was based on the initial tests described in the previous section. Three LS tests were conducted with the saltcake simulant, local soil, sand, and chemical additives $\left(\mathrm{B}_{2} \mathrm{O}_{3}\right.$ and $\left.\mathrm{ZrO}_{2}\right)$. Table 4.1 shows the composition of simulant, soil, and sand, expressed in oxides and halides that are expected to remain in glass. The glass component fraction in each material is also listed in Table 4.1. The balance includes all components that will leave the glass melt during processing. In addition, extra amount of flux and starter path materials were added to the top of the dried feed in the starter region of the melt.

Table 4.2 summarizes the amount of dried feed, flux, and starter path materials used in the LS-1 test. Note that the glass produced by this test contained only 10 mass $\% \mathrm{Na}_{2} \mathrm{O}$ to evaluate the effect of waste loading on the large-scale tests (glasses for subsequent two tests are formulated with progressively higher fractions of $\mathrm{Na}_{2} \mathrm{O}$ ). The flux material used in large-scale tests was different from those used in engineering-scale tests in that it did not contain a $\mathrm{Na}_{2} \mathrm{O}$ source. The flux material in engineering-scale tests was basically the glass composition without the waste component. Therefore, unlike in engineeringscale tests, the target glass composition is significantly affected by this flux material.

Calculated target compositions for LS-1 are shown in Table 4.3. The Target/Batch Feed column was calculated assuming that only the dried batch feed was converted into glass whereas the Target/Combined column was calculated assuming all the flux and starter path materials were dissolved in glass. A sample of glass was taken from LS-1 for the chemical composition by XRF and 7- and 14-day VHT.

Table 4.1. Composition of Simulant, Soil, and Sand Used in LS Tests

\begin{tabular}{|c|c|c|c|}
\hline Component & Simulant & Soil & Sand \\
\hline $\mathrm{Al}_{2} \mathrm{O}_{3}$ & 0.0188 & 0.1294 & 0.0119 \\
\hline $\mathrm{BaO}$ & & 0.0007 & 0.0002 \\
\hline $\mathrm{CaO}$ & & 0.0404 & \\
\hline $\mathrm{Cl}$ & 0.0090 & & \\
\hline $\mathrm{Cr}_{2} \mathrm{O}_{3}$ & 0.0046 & 0.0001 & \\
\hline $\mathrm{F}$ & 0.0035 & & \\
\hline $\mathrm{Fe}_{2} \mathrm{O}_{3}$ & & 0.0627 & 0.0016 \\
\hline $\mathrm{K}_{2} \mathrm{O}$ & 0.0034 & 0.0217 & 0.0014 \\
\hline $\mathrm{MgO}$ & & 0.0197 & 0.0003 \\
\hline $\mathrm{MnO}$ & & 0.0010 & 0.0001 \\
\hline $\mathrm{Na}_{2} \mathrm{O}$ & 0.8987 & 0.0272 & 0.0001 \\
\hline $\mathrm{P}_{2} \mathrm{O}_{5}$ & 0.0203 & 0.0021 & 0.0001 \\
\hline $\mathrm{SiO}_{2}$ & & 0.6829 & 0.9824 \\
\hline $\mathrm{SO}_{3}$ & 0.0418 & & 0.0001 \\
\hline $\mathrm{SrO}$ & & 0.0004 & \\
\hline $\mathrm{TiO}_{2}$ & & 0.0116 & 0.0010 \\
\hline $\mathrm{ZrO}_{2}$ & & & 0.0008 \\
\hline Total & 1.0000 & 1.0000 & 1.0000 \\
\hline Glass component fraction & 0.4539 & 0.9767 & 0.9928 \\
\hline
\end{tabular}


Table 4.2. Amount of Batch Feed, Flux, and Starter Path Materials Used in LS-1 Test

\begin{tabular}{||c|c|c||}
\hline Type & Material & Mass (kg) \\
\hline \multirow{4}{*}{ Dried batch feed } & Soil & 7110.9 \\
\cline { 2 - 3 } & Simulant & 2242.8 \\
\cline { 2 - 3 } & $\mathrm{ZrO}_{2}$ & 645.8 \\
\cline { 2 - 3 } & $\mathrm{B}_{2} \mathrm{O}_{3}$ & 455.8 \\
\cline { 2 - 3 } & $\mathrm{Subtotal}$ & 10455.3 \\
\hline \multirow{4}{*}{ Flux } & $\mathrm{Soil}_{1}$ & 2686.1 \\
\cline { 2 - 3 } & $\mathrm{ZrO}_{2}$ & 214.9 \\
\cline { 2 - 3 } & $\mathrm{B}_{2} \mathrm{O}_{3}$ & 150.7 \\
\cline { 2 - 3 } & $\mathrm{Subtotal}$ & 3051.7 \\
\hline Starter path & Soil & 185.0 \\
\hline \multicolumn{2}{|c|}{ Total } & 13692.0 \\
\hline
\end{tabular}

Table 4.3. Target and Measured Compositions of LS-1 Glass in Mass Fractions

\begin{tabular}{|c|c|c|c|}
\hline Component & $\begin{array}{c}\text { Target/ } \\
\text { Batch Feed }^{\text {(a) }}\end{array}$ & $\begin{array}{c}\text { Target/ } \\
\text { Combined }^{(b)}\end{array}$ & Measured \\
\hline $\mathrm{Al}_{2} \mathrm{O}_{3}$ & 0.1013 & 0.1047 & 0.0954 \\
\hline $\mathrm{B}_{2} \mathrm{O}_{3}$ & 0.0503 & 0.0496 & 0.0374 \\
\hline $\mathrm{BaO}$ & 0.0006 & 0.0006 & \\
\hline $\mathrm{CaO}$ & 0.0310 & 0.0322 & 0.0260 \\
\hline $\mathrm{Cl}$ & 0.0010 & 0.0007 & 0.0004 \\
\hline $\mathrm{Cr}_{2} \mathrm{O}_{3}$ & 0.0006 & 0.0005 & 0.0004 \\
\hline $\mathrm{F}$ & 0.0004 & 0.0003 & \\
\hline $\mathrm{Fe}_{2} \mathrm{O}_{3}$ & 0.0480 & 0.0500 & 0.0435 \\
\hline $\mathrm{K}_{2} \mathrm{O}$ & 0.0170 & 0.0175 & 0.0117 \\
\hline $\mathrm{MgO}$ & 0.0151 & 0.0157 & 0.0227 \\
\hline $\mathrm{MnO}$ & 0.0008 & 0.0008 & \\
\hline $\mathrm{Na}_{2} \mathrm{O}$ & 0.1217 & 0.0964 & 0.0836 \\
\hline $\mathrm{P}_{2} \mathrm{O}_{5}$ & 0.0039 & 0.0034 & 0.0027 \\
\hline $\mathrm{SiO}_{2}$ & 0.5233 & 0.5442 & 0.5669 \\
\hline $\mathrm{SO}_{3}$ & 0.0047 & 0.0035 & 0.0026 \\
\hline $\mathrm{SrO}$ & 0.0003 & 0.0003 & \\
\hline $\mathrm{TiO}_{2}$ & 0.0089 & 0.0092 & 0.0075 \\
\hline $\mathrm{ZrO}_{2}$ & 0.0712 & 0.0703 & 0.0717 \\
\hline Total & 1.0000 & 1.0000 & 0.9725 \\
\hline Soil & 0.7662 & 0.7969 & \\
\hline Simulant & 0.1123 & 0.0832 & \\
\hline Additive & 0.1215 & 0.1199 & \\
\hline \multicolumn{4}{|c|}{$\begin{array}{l}\text { (a) Calculated assuming only the dried batch feed was convertec } \\
\text { to glass. } \\
\text { (b) Calculated assuming that all the flux and starter path } \\
\text { materials were dissolved into glass. }\end{array}$} \\
\hline
\end{tabular}




\subsection{Chemical Analysis and Phase Identification}

The result of one representative XRF analysis of the LS-1 glass sample is in Table 4.3.

Attention was also paid to the silica lining separating the container refractory walls from the melt. The initial silica-sand lining was approximately $25 \mathrm{~cm}$ thick before melting began. During the melting process, the lining was permeated with molten glass to the depth of 15 to $50 \mathrm{~mm}$ depending on the position in the block. Generally, the depth of melt penetration decreased with the vertical distance above the block bottom (see Figure 4.1). Several transition layers formed at the sand side of the interface between the silica-sand lining and the glass block. The sand-glass interfacial layer had five major zones: black bulk melt, gray sand-melt layer, blue sand-melt layer, yellow sand-melt layer, and pure white sand layer. This configuration was typical for the bottom and lower portion of the glass block side-walls. Higher on the sidewalls, the yellow layer was generally absent.

As Figure 4.2 shows, the top surface of the large-scale melt was a result of the collapse of large offgas bubbles from the melting reactions. A sample of the top surface is shown in Figure 4.3 indicating locations from which thin sections were prepared.

The sample in Figure 4.3 contains several areas. XRD analysis was performed for the grainy LS-1-B-R sample shown in Figure 4.4. Silica, hematite, and anorthite are probably unreacted soil minerals; zircon is a reaction product of zirconia with silica sand from the soil. The LS-1-B-F sample from the glassy area with large bubbles contains a large fraction of crystalline phase (Figure 4.5). Finally, Figure 4.6 (LS-1-BG sample) shows that the glass phase penetrated into the sand layer.

Figure 4.7 shows samples of a red compact material and a tan porous melt, both from the top portion of the block - see the results of XRD analysis in Figure 4.8 and Figure 4.9. The XRD analysis of these samples indicates that their crystalline portion (silica, albite, and anorthite) originated from the soil and added zirconia (baddeleyite). Figure 4.10 shows results of XRD analysis of a lava-like sample (LS-1-F) also from the top portion of the block. The crystalline fraction in this sample consists of residual silica and baddeleyite. A single sample from the block bottom (Figure 4.11) contains metallic droplets, similar to those seen in the engineering-scale melts (ES-1 and ES-2). 

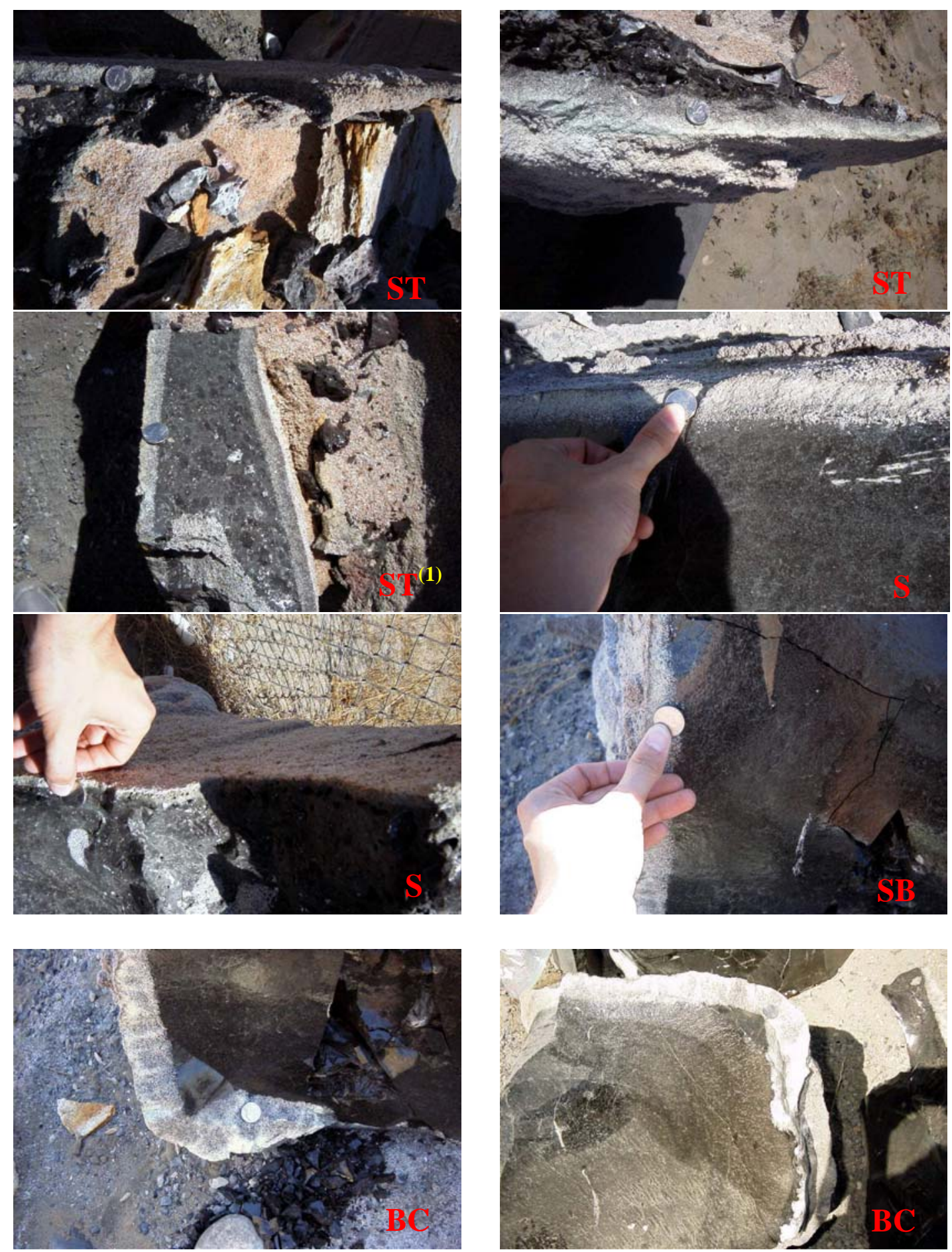

Figure 4.1. Interfacial Area between Silica-Sand Lining and LS-1 Glass Block.

The Interface Thickness is Indicated with a U.S. Nickel. The Position with Respect to the Block: BC Bottom corner, S Sidewall, SB Sidewall near the bottom, ST Sidewall near the top Note: A portion of the top material including the sand insulating layer has been drawn into the melt next to the sidewall 


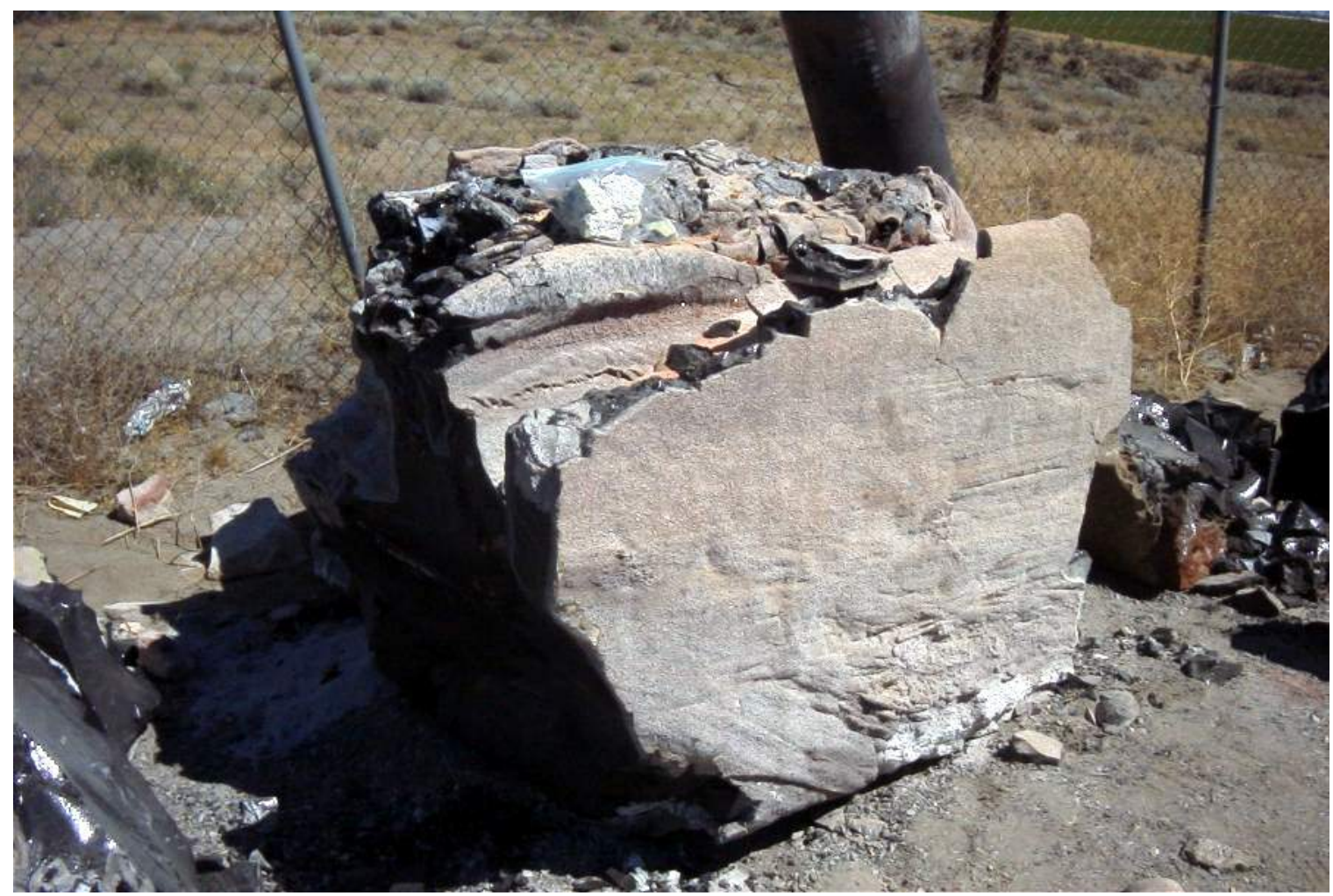

Figure 4.2. A Fraction of the LS-1 Block with the Top Surface

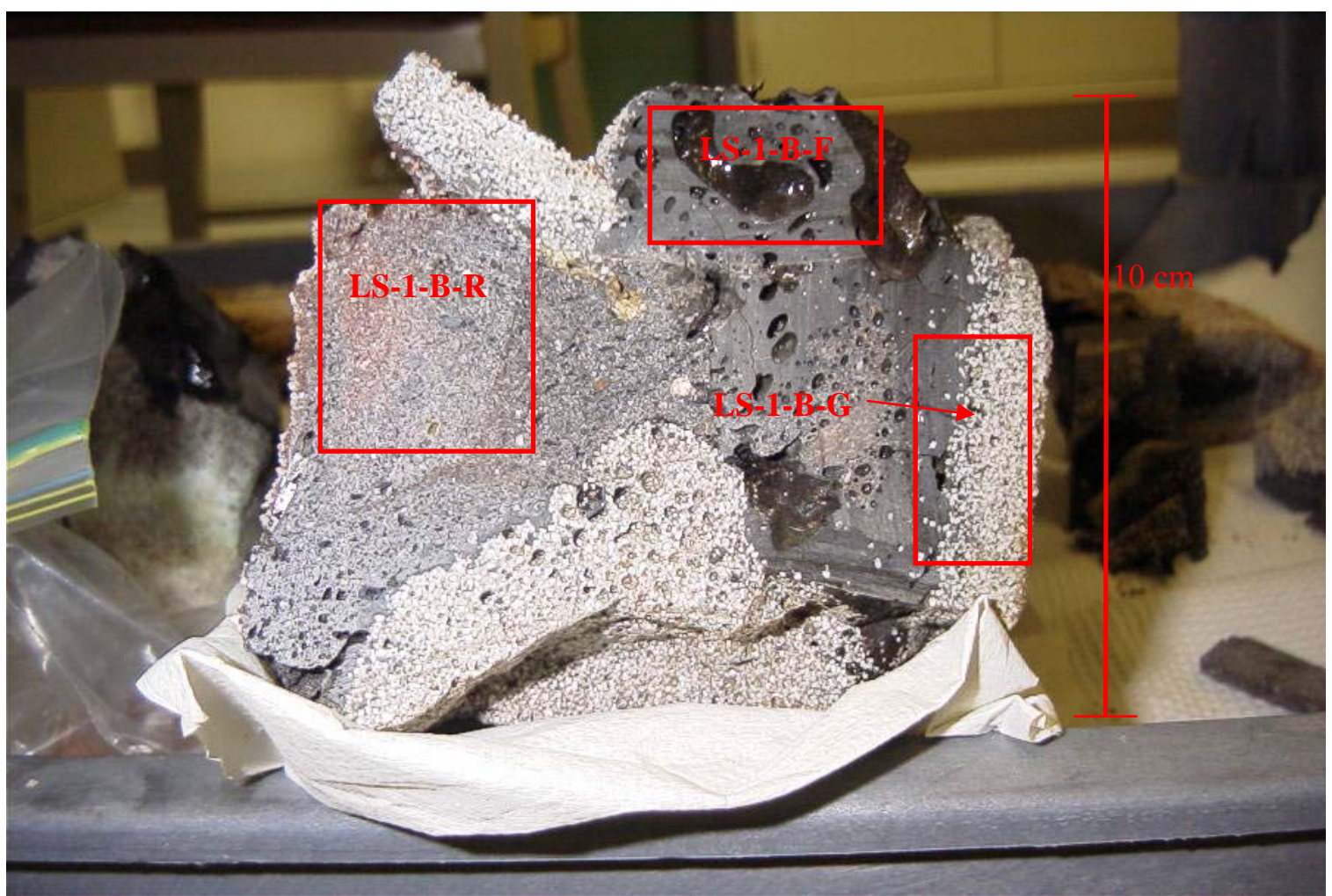

Figure 4.3. LS-1-B Sample Taken From the Top of LS-1 Block (see Figure 4.2) 

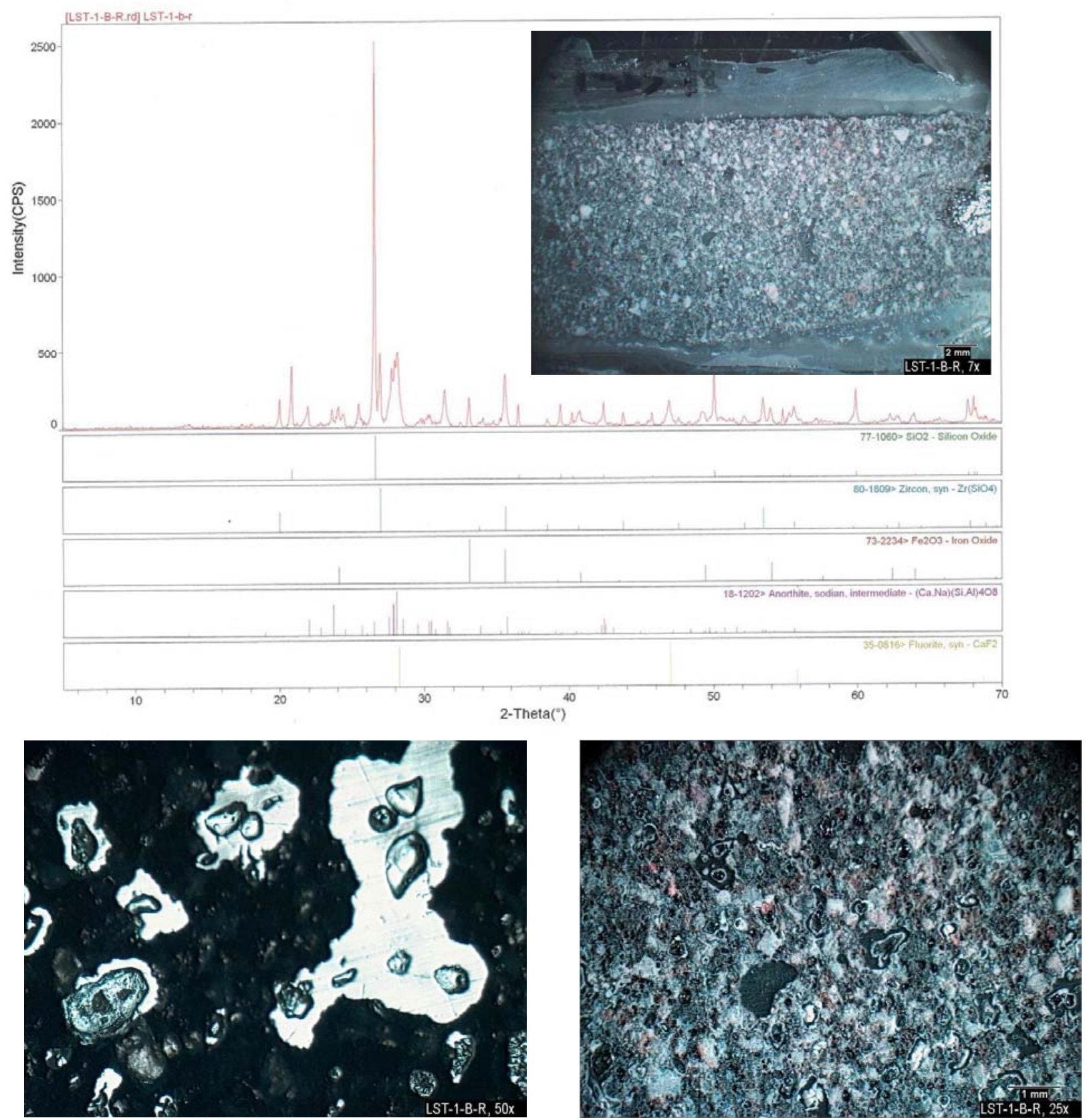

Figure 4.4. XRD and Optical Micrographs of LS-1-B-R Sample (purple area, see Figure 4.3) (inset and bottom right reflected light, bottom left transmitted light showing voids in the material) 

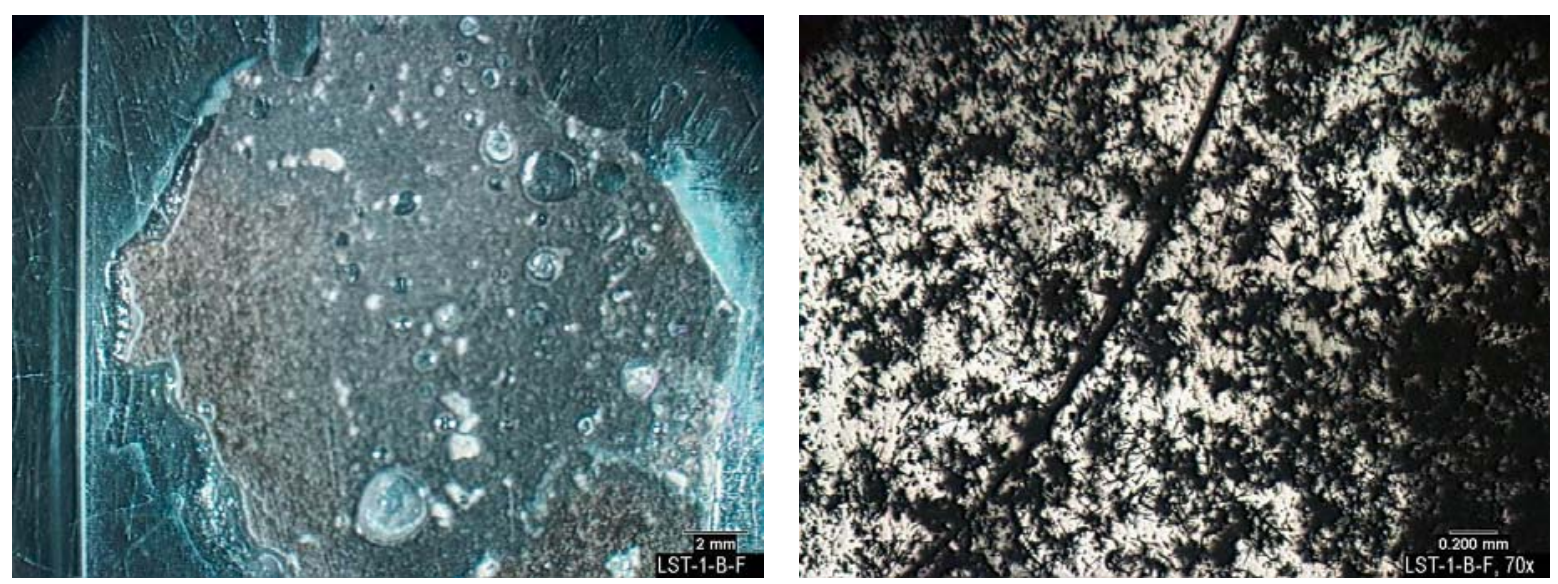

Figure 4.5. Optical Micrographs of LS-1-B-F sample (see Figure 4.3) Showing Amorphous phase with the Formation of Unidentified Crystals
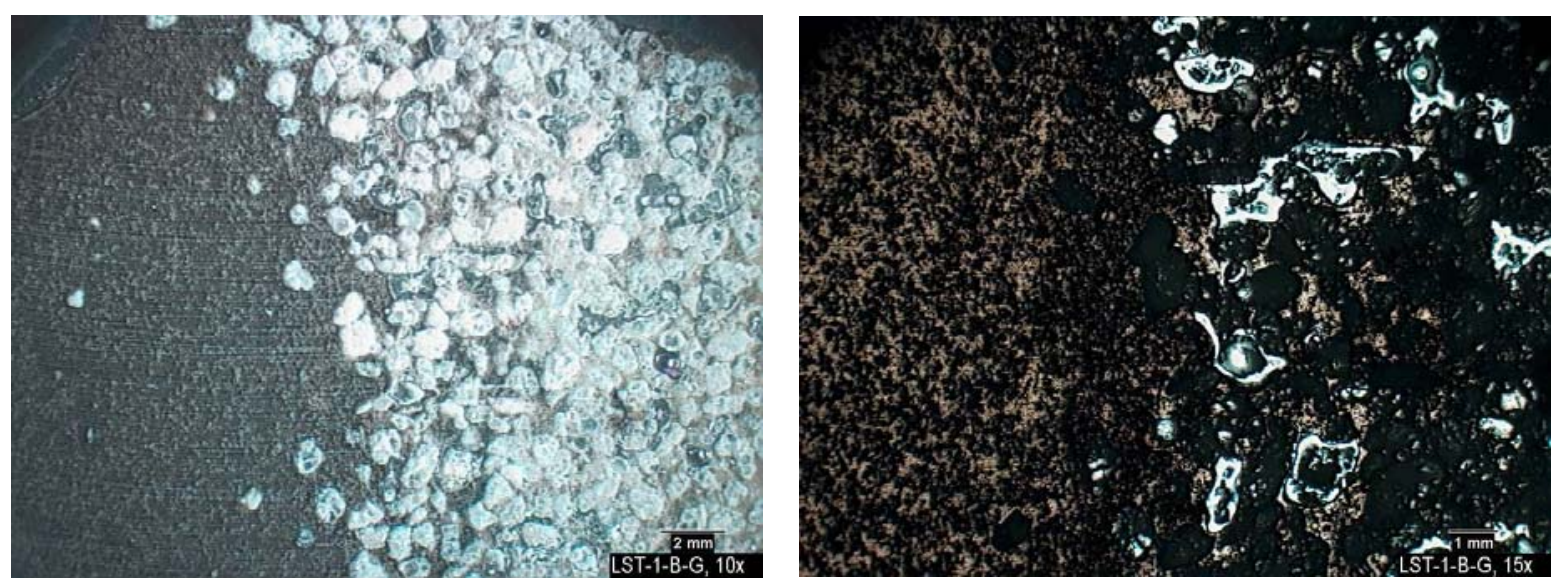

Figure 4.6. Optical Micrographs of LS-1-B-G Sample (see Figure 4.3) Showing the Sand-Glass Interface and Crystal Formation in Glass 


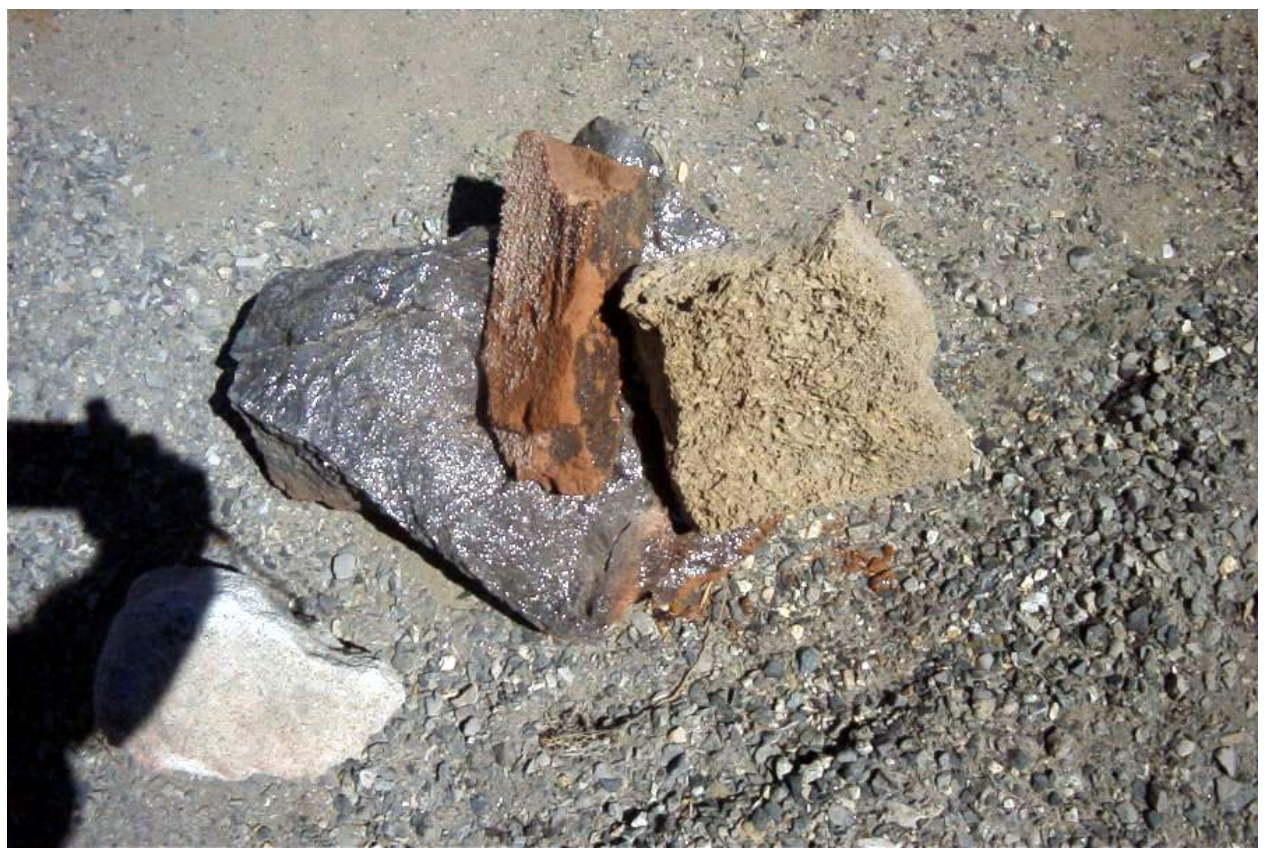

Figure 4.7. Large $(\sim 30-\mathrm{cm})$ Pieces of Material from the Top Portion of the LS-1 Block

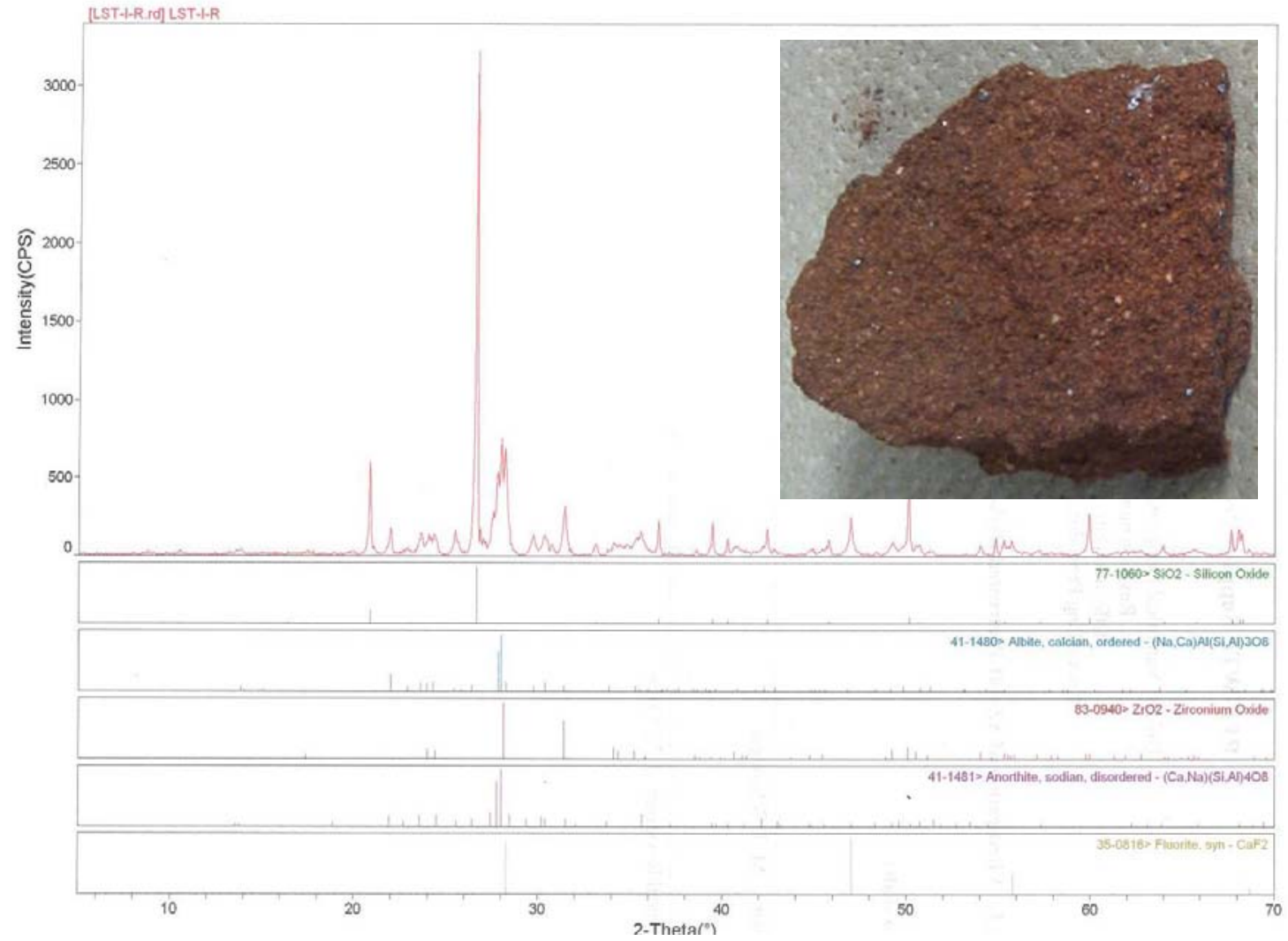

Figure 4.8. XRD Analysis of LS-1-R, the Red Sample Shown in Figure 4.7 


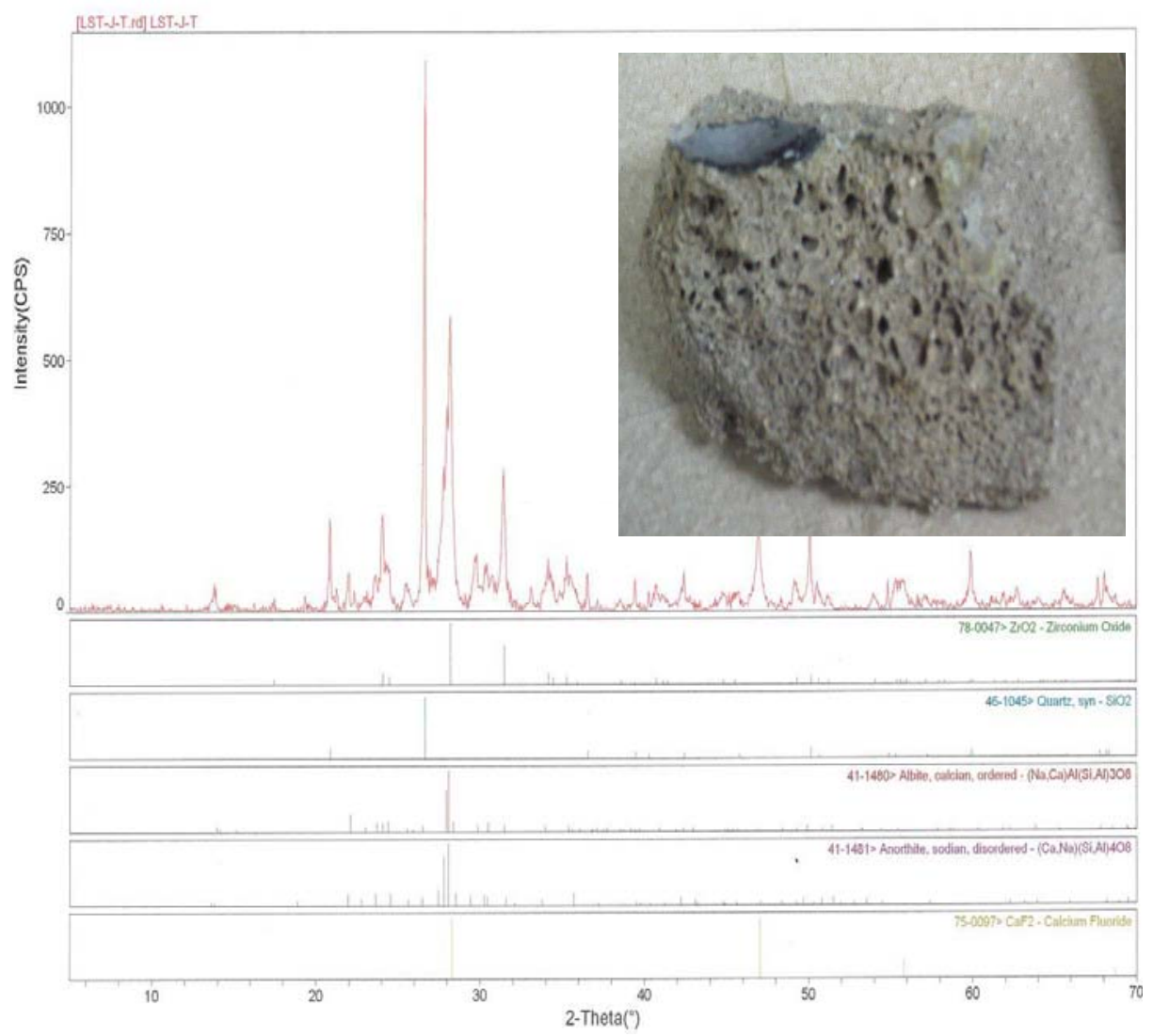

Figure 4.9. XRD Analysis of LS-1-T, the Tan Sample Shown in Figure 4.7

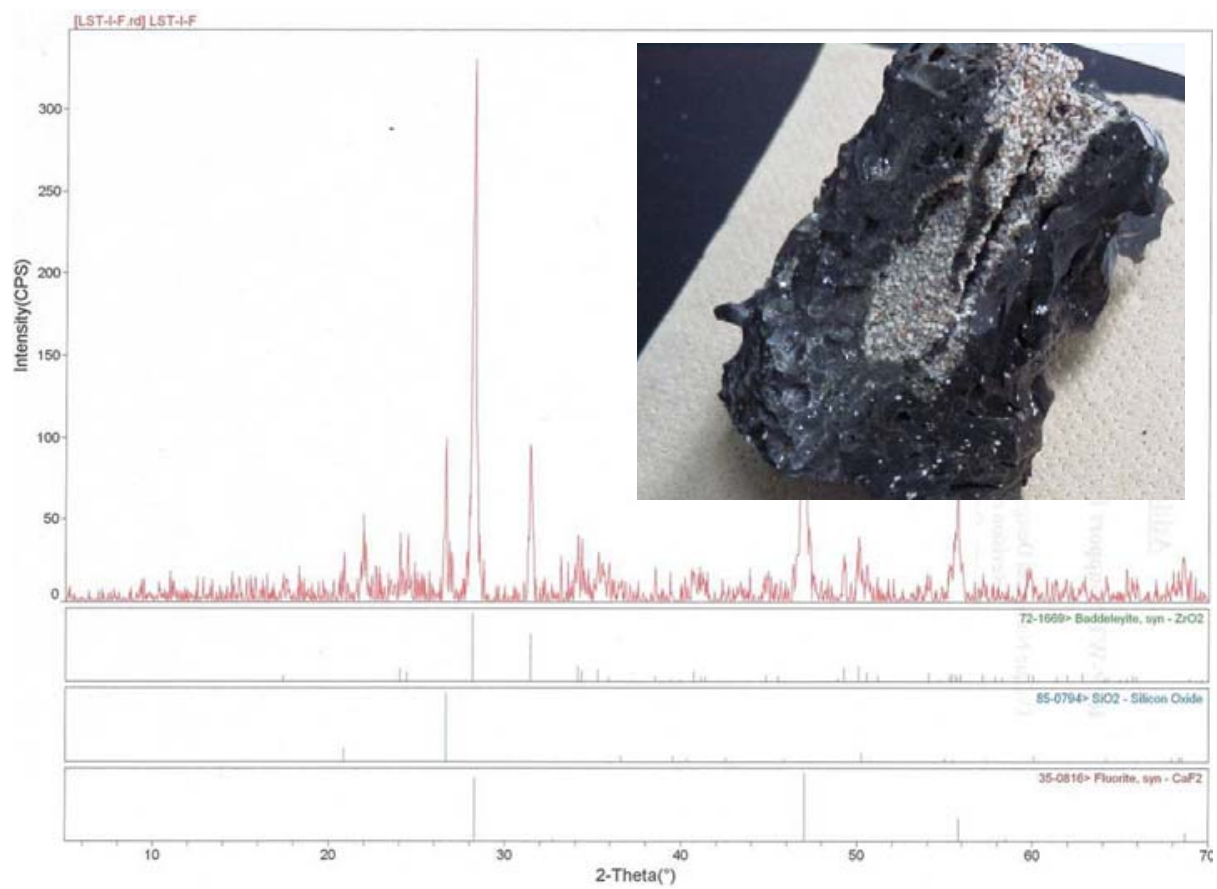

Figure 4.10. XRD Analysis of LS-1-F Sample from the Foamy Layer on the Top of the LS-1 Block 

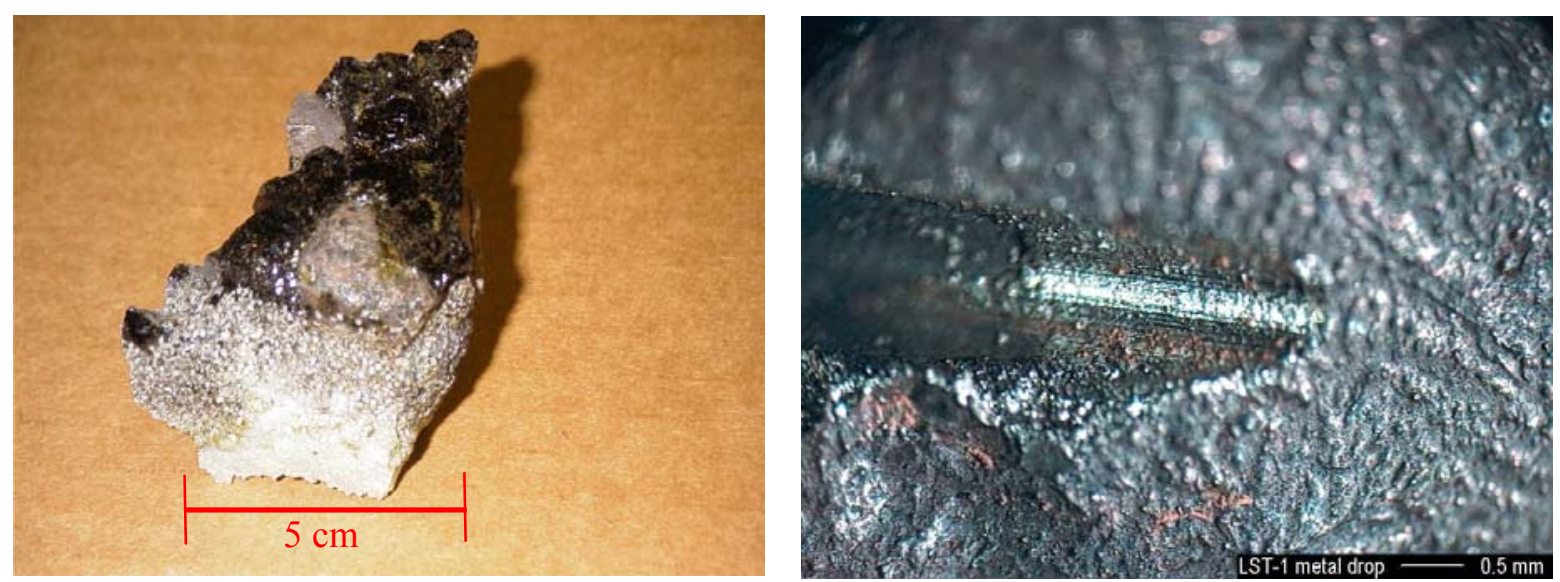

Figure 4.11. A Sample of LS-1 Glass from the Block Bottom with Metallic Droplets. Massive Droplets (over $2 \mathrm{~cm}$ in diameter) are Visible on the Left Photograph, the Rough Surface of which is Displayed on the Stereo-Microscope Image on the Right

A portion of the yellow layer at the silica-sand-glass interface was soaked in DI water and the solution (see inset in Figure 4.12) was subsequently evaporated. The XRD pattern of the precipitate is shown in Figure 4.12, identifying sodium sulfate salts.

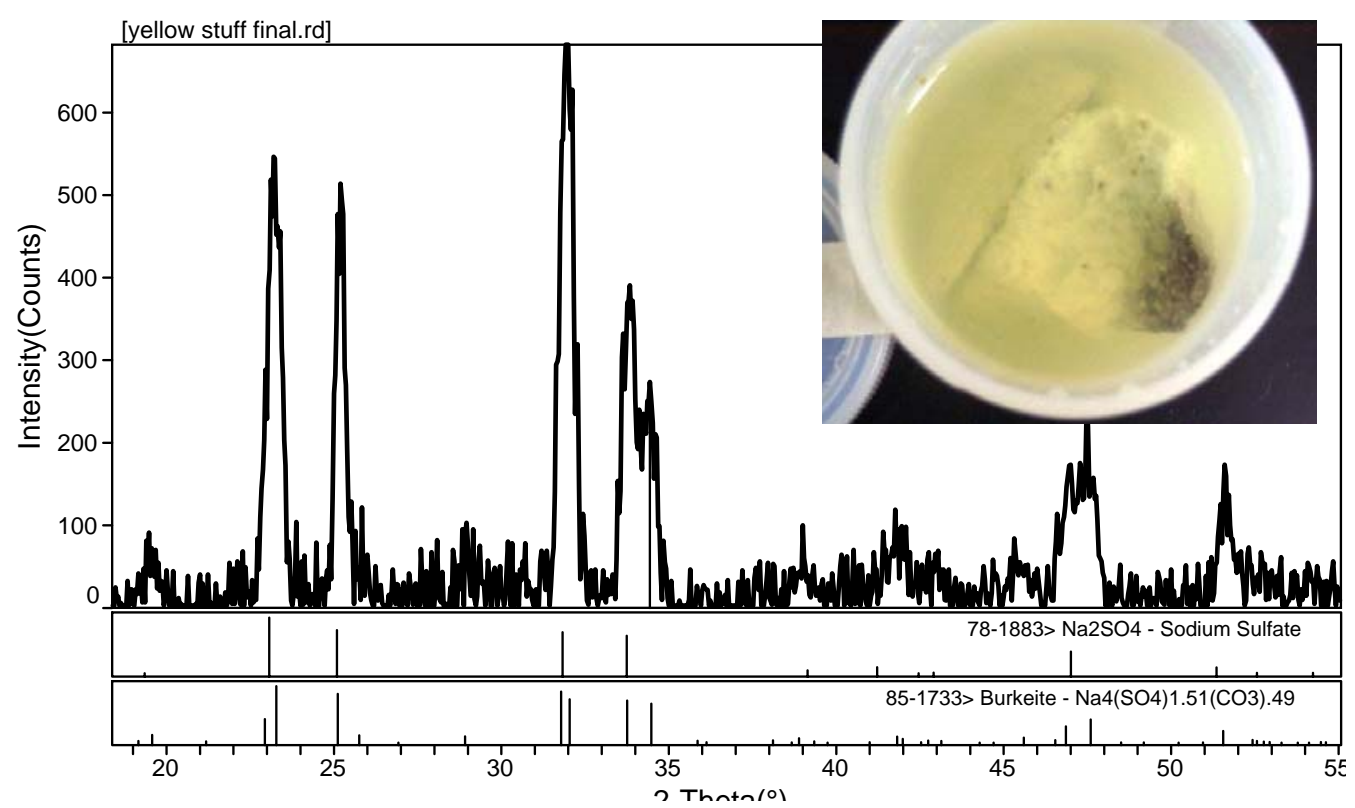

Figure 4.12. XRD Pattern of Precipitate from the LS-1 Yellow Layer Leachate (shown in the inset)

An XRF chemical analysis of a blue layer sample (Figure 4.13) is in Table 4.4. Using the mass balance and the compositions of the soil, fractions of basic components in the blue layer were estimated. The soil fraction of 0.047 was obtained assuming that the soil was the single source of $\mathrm{CaO}, \mathrm{MgO}$, and $\mathrm{Fe}_{2} \mathrm{O}_{3}$ in the blue layer. Calculation the $\mathrm{ZrO}_{2}$ fraction was straightforward because added zirconia was its only source. The sand fraction (0.877) was obtained by subtracting from the overall silica content the portion supplied by the soil (the sand was $100 \%$ silica). The waste fraction was than the remaining material that 
was not soil, silica, or zirconia. Table 4.5 shows the results of calculations. The fractions in Table 4.5 do not include $\mathrm{B}_{2} \mathrm{O}_{3}$, the only component that was not available from the XRF analysis. The result shows that the major component of the blue layer was silica sand and only 12.3 mass $\%$ was glass. The calculated waste composition roughly corresponds to the composition of the waste simulant used (see Table 4.6).

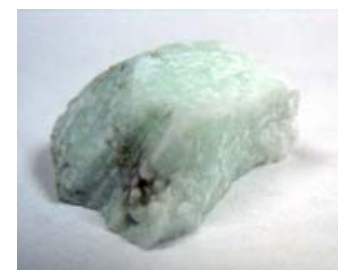

Figure 4.13. Blue Layer Sample Analyzed in Table 4.4

Table 4.4. Measured Composition of the Blue Sample and Estimated Composition of the Salt in the Sample

\begin{tabular}{||c|c||}
\hline Component & $\begin{array}{c}\text { Blue } \\
\text { layer }\end{array}$ \\
\hline $\mathrm{Al}_{2} \mathrm{O}_{3}$ & 0.0185 \\
\hline $\mathrm{B}_{2} \mathrm{O}_{3}$ & $\mathrm{ND}$ \\
\hline $\mathrm{CaO}$ & 0.0013 \\
\hline $\mathrm{Cl}$ & 0.0008 \\
\hline $\mathrm{Cr}_{2} \mathrm{O}_{3}$ & 0.0002 \\
\hline $\mathrm{F}$ & 0.0000 \\
\hline $\mathrm{Fe}_{2} \mathrm{O}_{3}$ & 0.0046 \\
\hline $\mathrm{K}_{2} \mathrm{O}$ & 0.0049 \\
\hline $\mathrm{MgO}$ & 0.0017 \\
\hline $\mathrm{Na}_{2} \mathrm{O}$ & 0.0534 \\
\hline $\mathrm{P}_{2} \mathrm{O}_{5}$ & 0.0011 \\
\hline $\mathrm{SiO}_{2}$ & 0.8996 \\
\hline $\mathrm{SO}_{3}$ & 0.0012 \\
\hline $\mathrm{TiO}_{2}$ & 0.0018 \\
\hline $\mathrm{ZrO}_{2}$ & 0.0041 \\
\hline $\mathrm{Sum}^{2}$ & 0.9931 \\
\hline
\end{tabular}

ND Not determined

Table 4.5. Estimated Mass Fractions of Batch Materials in the Blue Layer Sample

\begin{tabular}{||l|c||}
\hline \hline Soil & 0.0466 \\
\hline Salts from waste & 0.0726 \\
\hline $\mathrm{ZrO}_{2}$ & 0.0041 \\
\hline Silica sand & 0.8767 \\
\hline
\end{tabular}


Table 4.6. Used and Calculated Compositions of Waste in the Blue Layer Sample

\begin{tabular}{||l|c|c||}
\hline Component & Target & Calculated \\
\hline $\mathrm{Al}_{2} \mathrm{O}_{3}$ & 0.019 & 0.167 \\
\hline $\mathrm{Cl}$ & 0.009 & 0.012 \\
\hline $\mathrm{Cr}_{2} \mathrm{O}_{3}$ & 0.005 & 0.003 \\
\hline $\mathrm{F}$ & 0.004 & \\
\hline $\mathrm{K}_{2} \mathrm{O}$ & 0.003 & 0.052 \\
\hline $\mathrm{Na}_{2} \mathrm{O}$ & 0.898 & 0.720 \\
\hline $\mathrm{P}_{2} \mathrm{O}_{5}$ & 0.020 & 0.013 \\
\hline $\mathrm{SO}_{3}$ & 0.042 & 0.017 \\
\hline $\mathrm{TiO}_{2}$ & & 0.016 \\
\hline $\mathrm{Sum}$ & 1.000 & 1.000 \\
\hline
\end{tabular}

Optical micrographs of the transition layers between glass and silica-sand lining are in Figure 4.14 through Figure 4.19. Figure 4.14 shows the layer arrangement from glass through the gray, blue, and yellow layers to the white silica. Details of the individual layers are in Figure 4.15 through Figure 4.19. Figure 4.15 shows the baddeleyite crystals in the glass adjacent to the interface with silica. Baddeleyite precipitated from the glass on cooling when the melt became oversaturated with $\mathrm{ZrO}_{2}$ as a result of an increase concentration of $\mathrm{SiO}_{2}$. Inside the silica sand lining, the glass was saturated with $\mathrm{SiO}_{2}$ that precipitated on cooling in the form tridymite needles as seen in Figure 4.16. The blue layer (Figure 4.17) was a mixture of silica particles, glass and pores. The images of the blue, yellow, and white layers (Figure 4.17 through Figure 4.19) are somewhat obscured by the epoxy resin used to keep the sample together.

The SEM EDS analyses of the blue and yellow layers are shown in Figure 4.20 through Figure 4.22, which show the presence of additional minerals, such as albite, diopside, tridymite, and cristobalite.

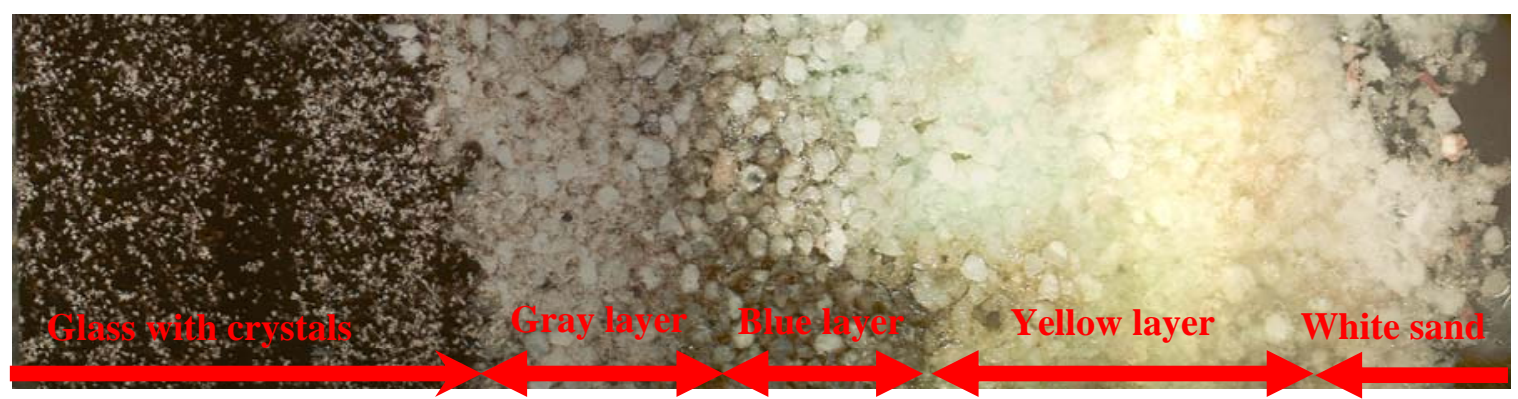

Figure 4.14. Cross Section through Glass-Silica Sand Interface (the thickness from let to right is 58 mm) 


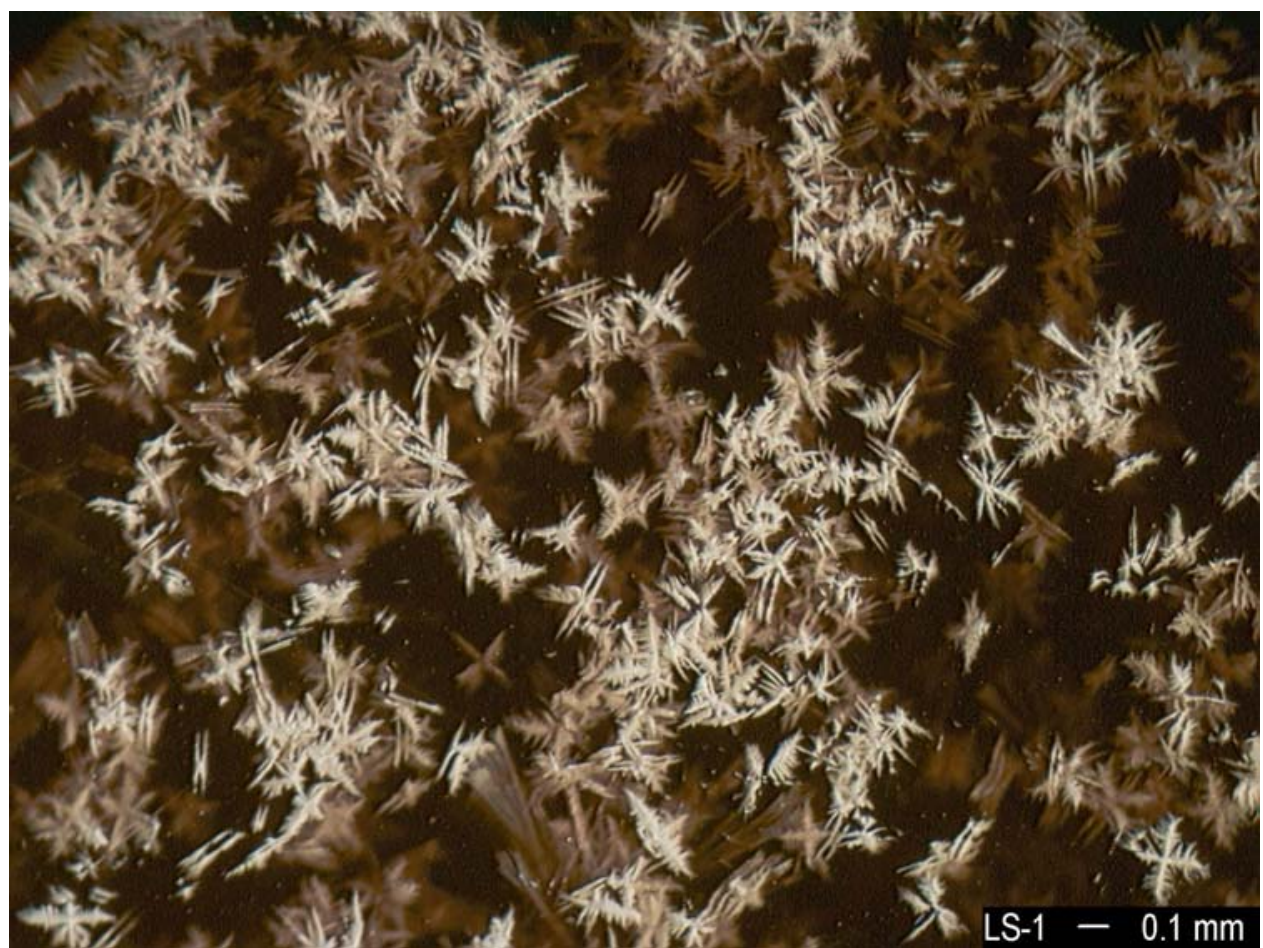

Figure 4.15. Glass with White Crystals of Baddeleyite Adjacent to the Interface with Silica

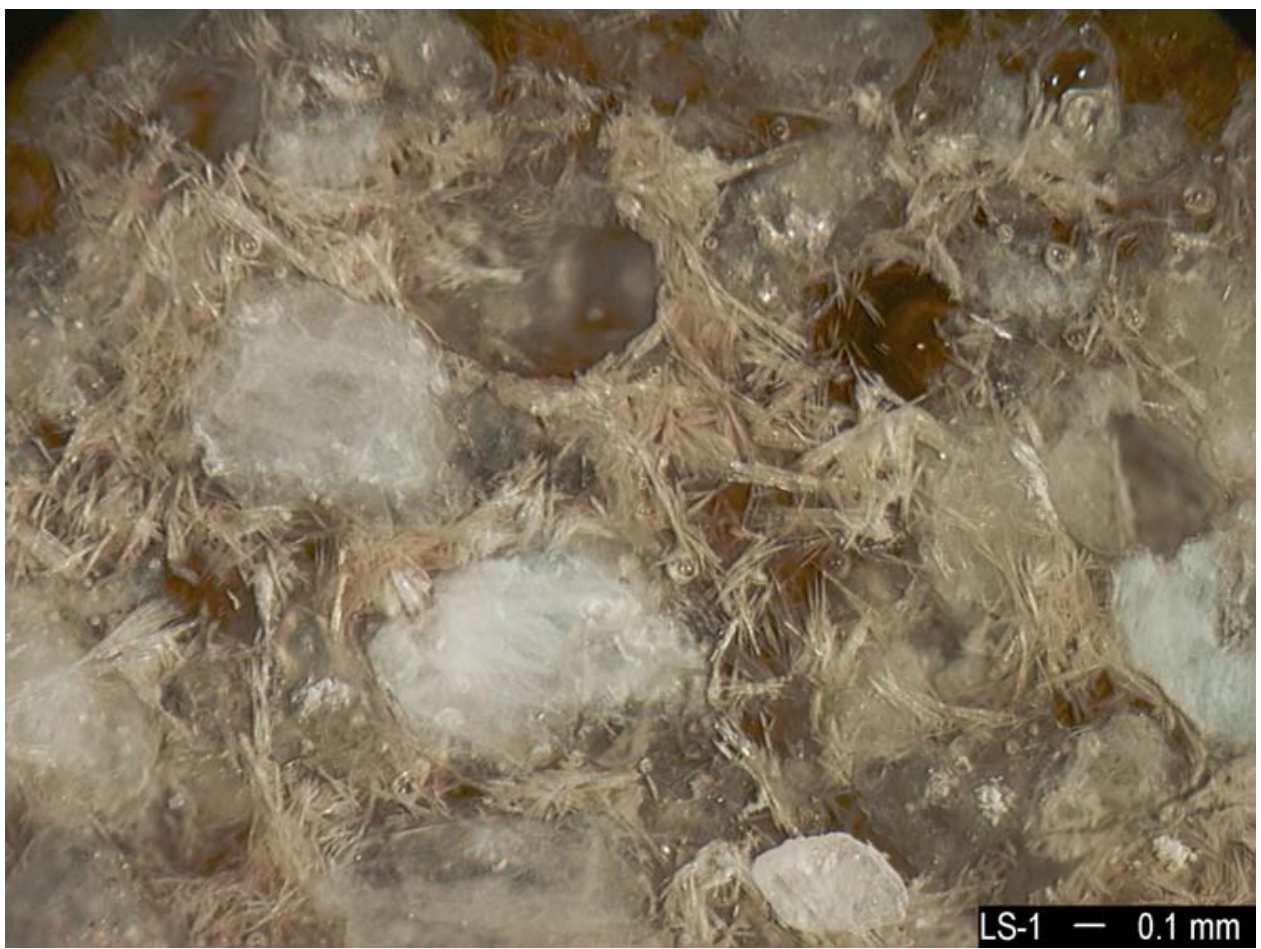

Figure 4.16. Silica Particles Permeated with Glass and Tridymite Crystals in the Gray Layer 


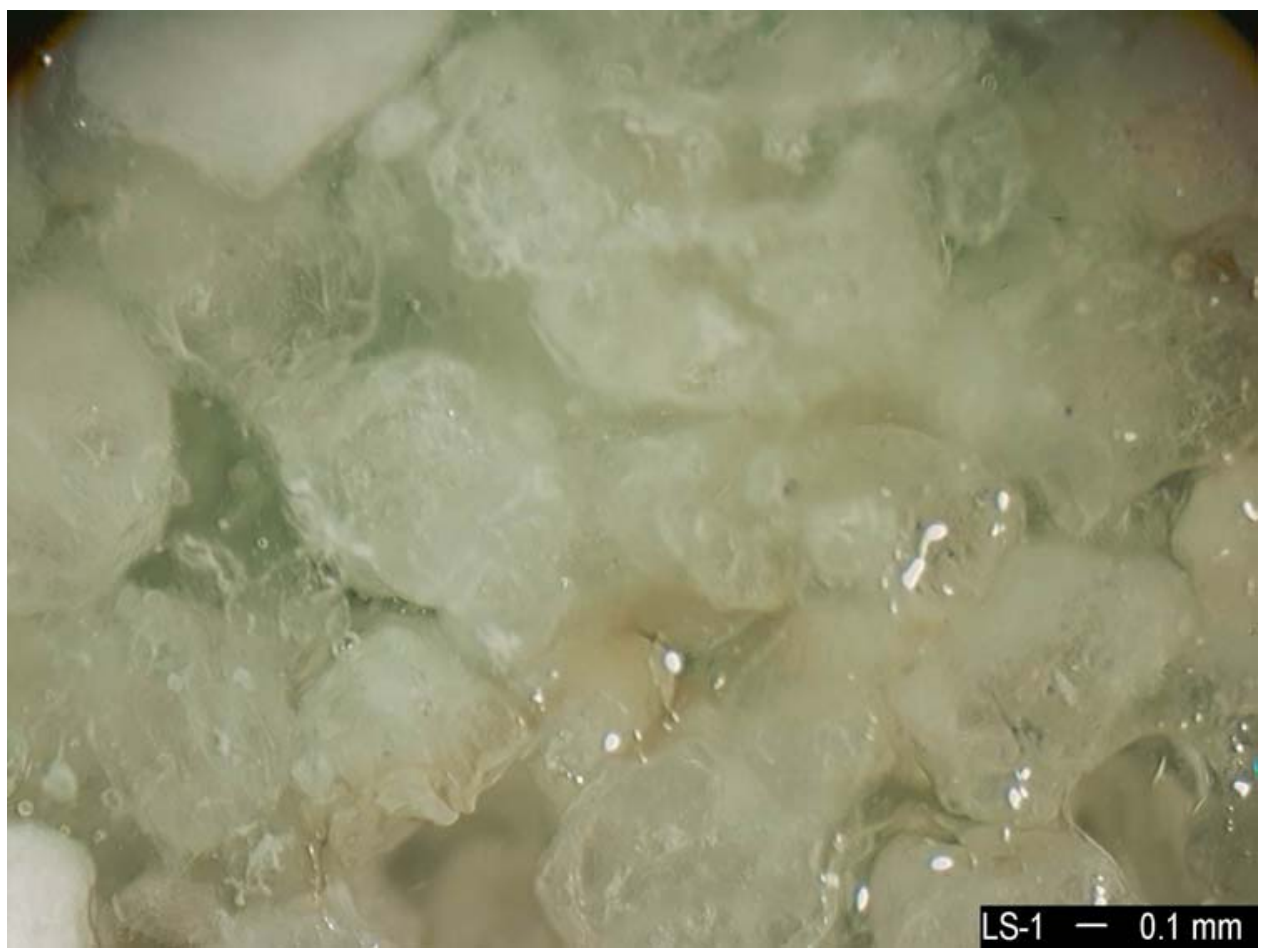

Figure 4.17. Silica Particles with Glass and Pores in the Blue Layer

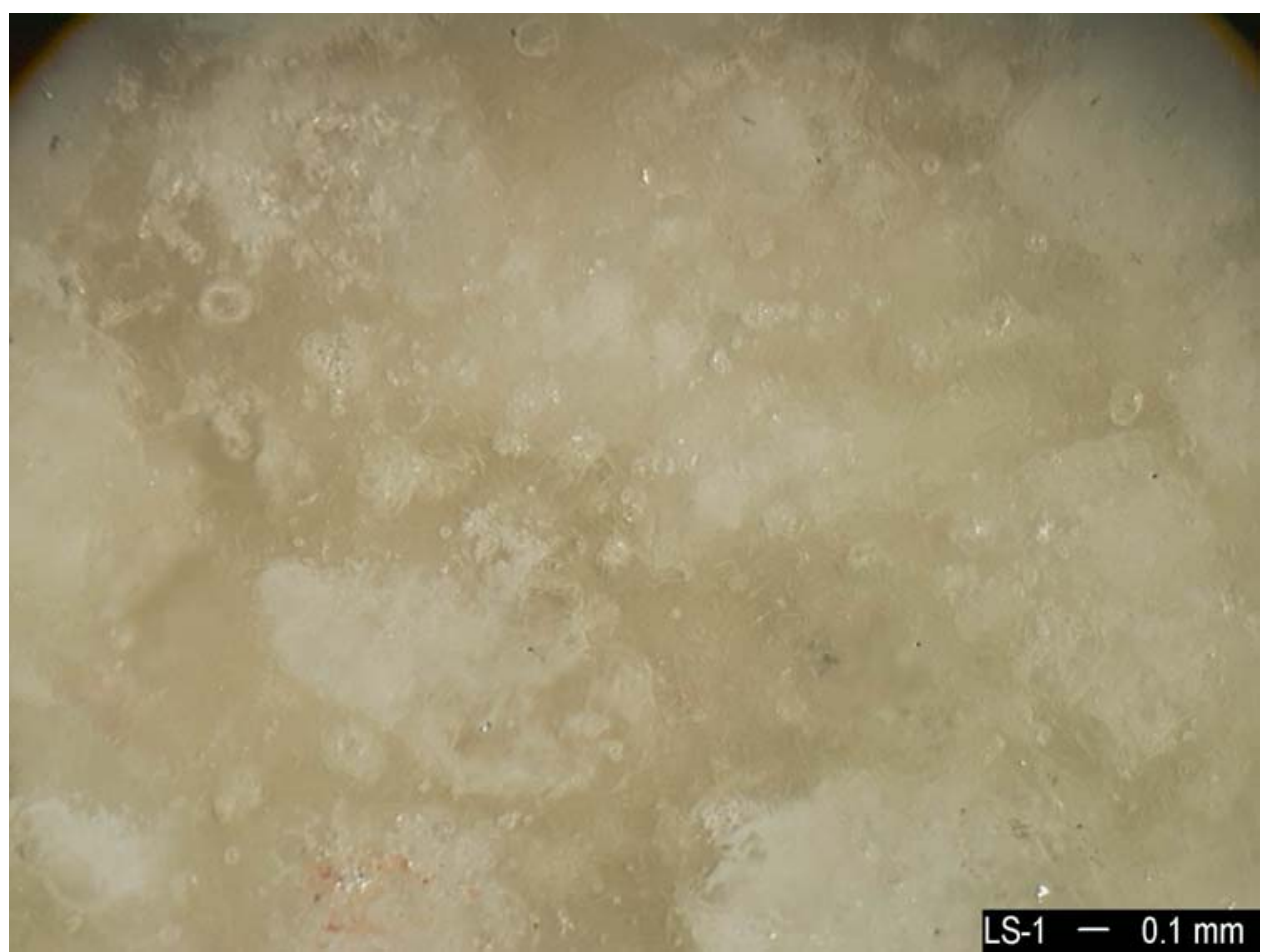

Figure 4.18. Silica Particles with Glass and Pores in the Yellow Layer 


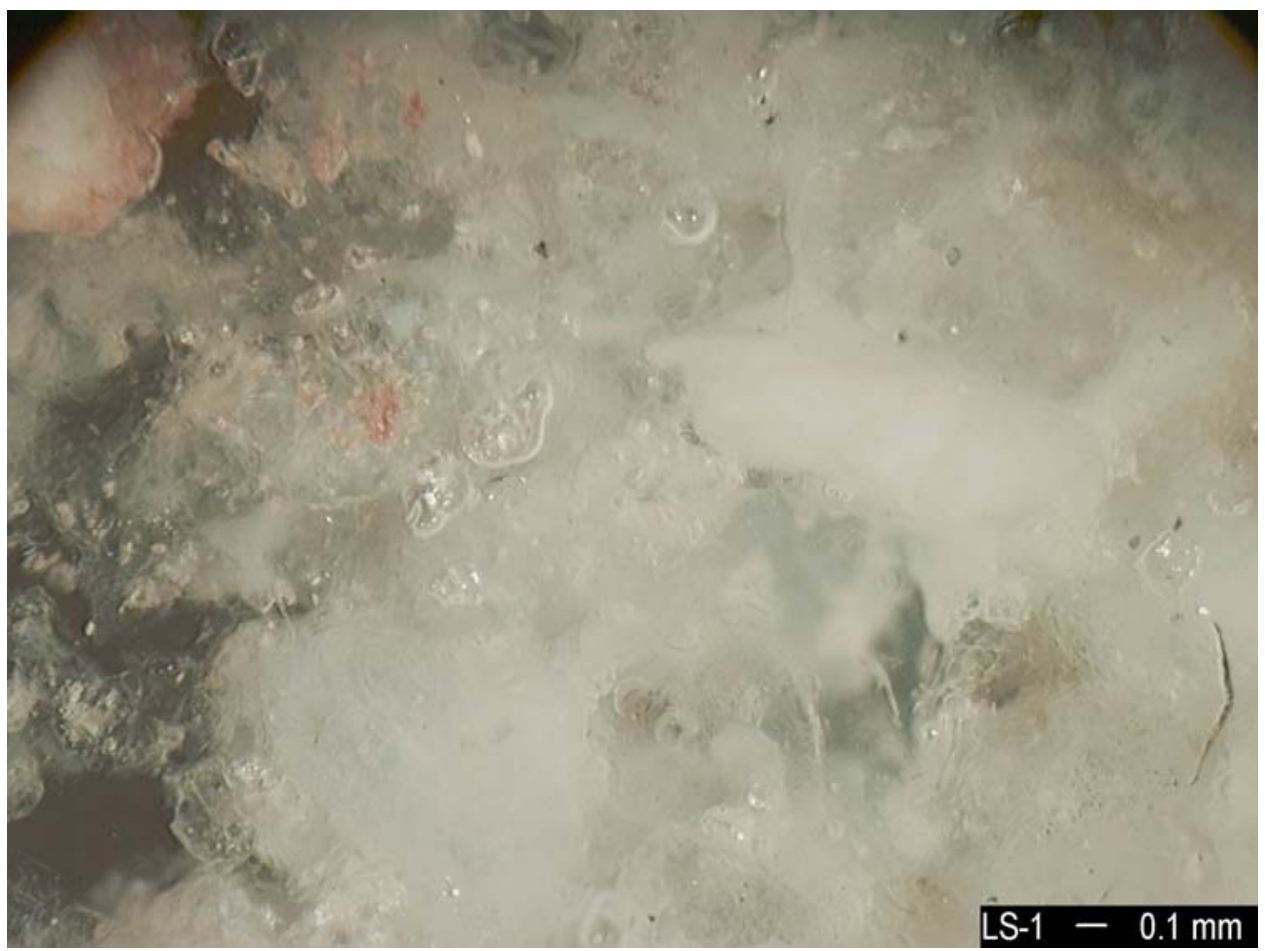

Figure 4.19. Silica Particles in the White Layer

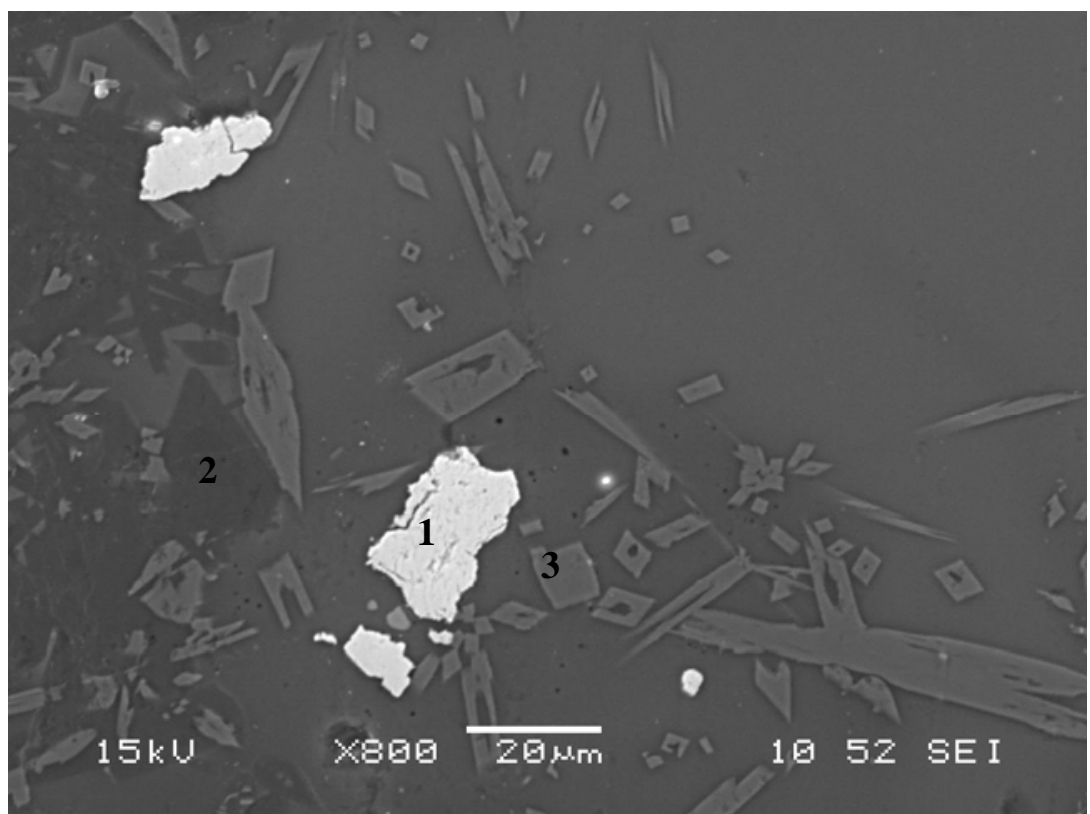

Figure 4.20. SEM Micrograph of the Amorphous Phase in the LS-1 Yellow Layer with Undissolved Zirconia (1), Albite $\left(\mathrm{NaAlSi}_{3} \mathrm{O}_{8}\right)$ (2) and Diopside (CaMg. $\left.\mathrm{Fe}_{0.3} \mathrm{Si}_{2} \mathrm{O}_{6}\right)$ (3) 


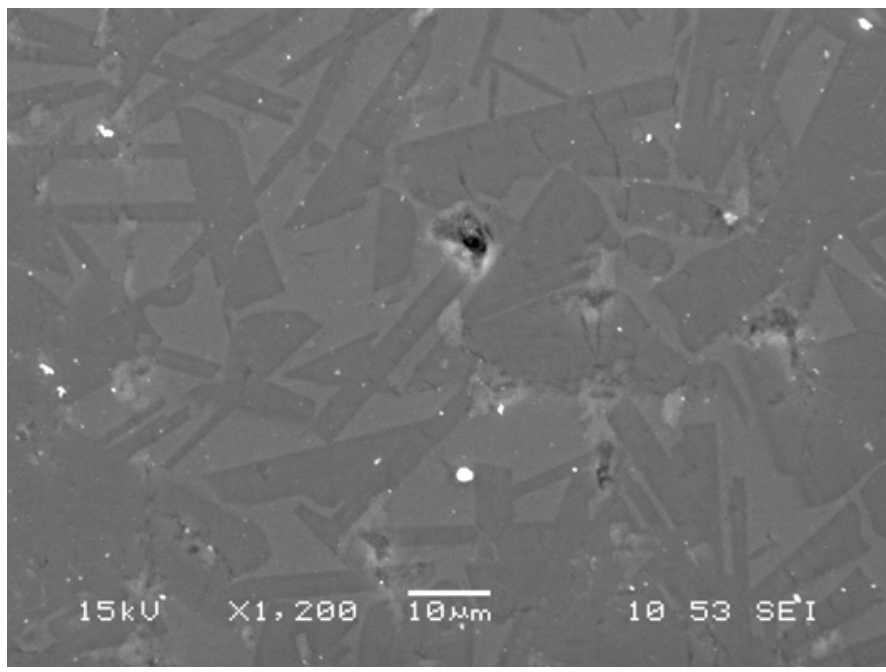

Figure 4.21. SEM Micrograph of LS-1 Blue Layer with Crystals of Tridymite $\left(\mathrm{SiO}_{2}\right)$

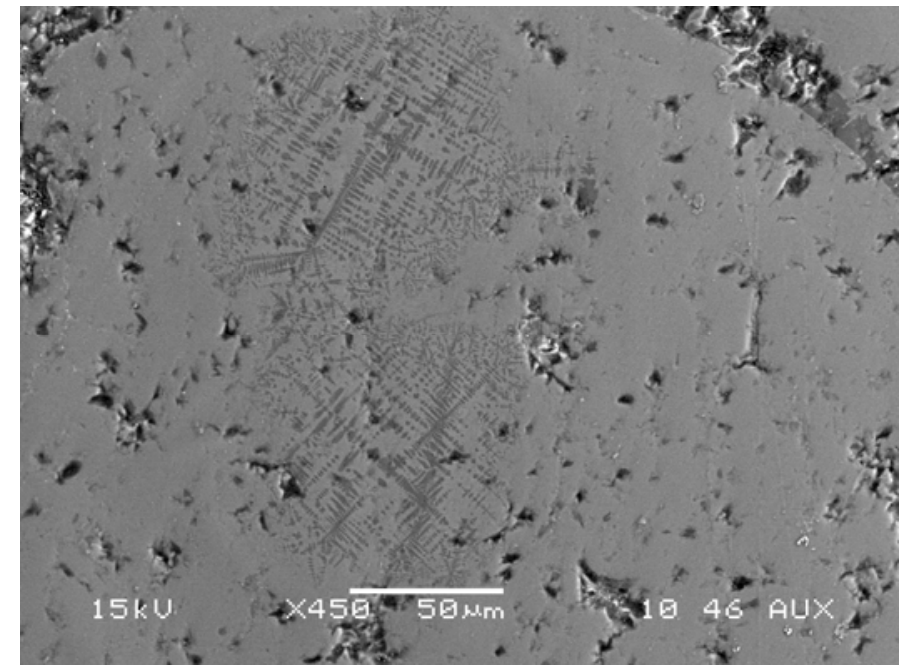

Figure 4.22. SEM Micrograph of LS-1 Blue Layer with Crystals of Cristobalite $\left(\mathrm{SiO}_{2}\right)$

\subsection{Vapor Hydration Test and Toxicity Characteristic Leach Procedure}

Two representative samples were subjected to the VHT for 7- and 14-days. The "snow-like" crystalline inclusions are seen in the pre-tested sample coupon shown in Figure 4.23. Figure 4.24 shows the cross sections of the two VHT specimens after testing for 7- and 14-days. There is no visible sign of alteration. Only crystalline inclusions in unaltered glass can be seen. Table 4.7 lists the measured VHT responses and calculated alteration rates for the two specimens. The VHT results from these samples confirmed that the product was highly durable (e.g., resistant to the VHT, which is the most challenging chemical durability test for LAW glasses to meet). 


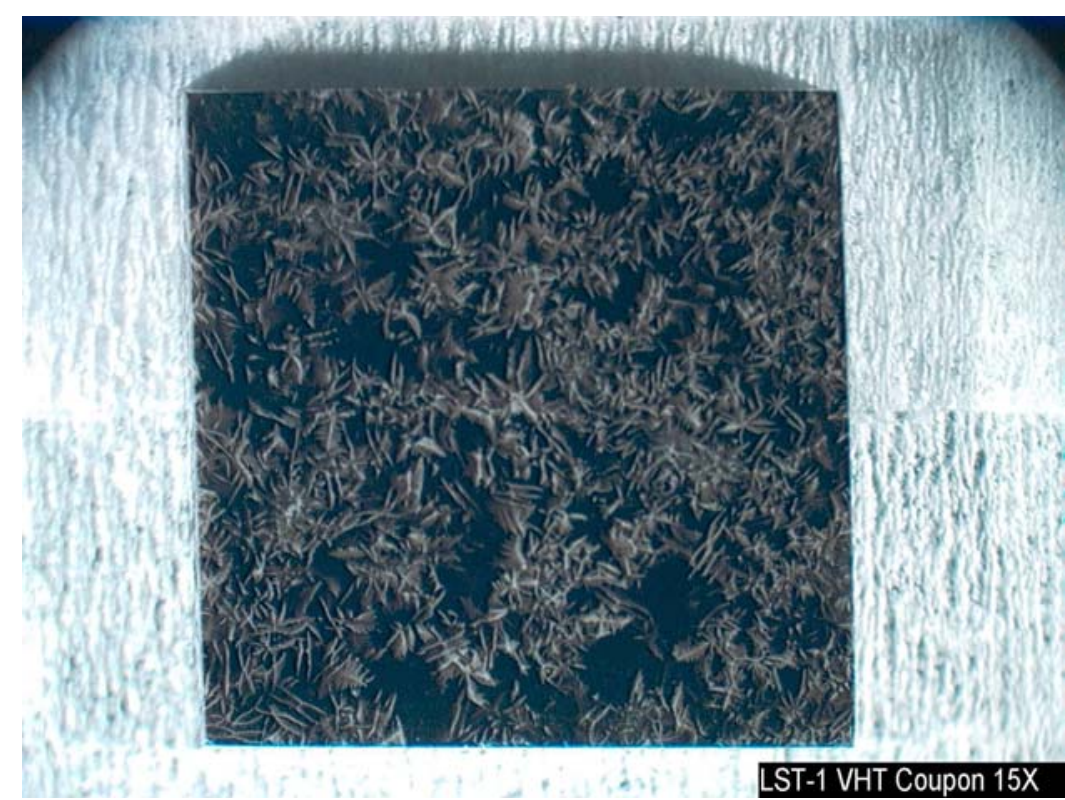

Figure 4.23. Optical Micrograph of LST-1 VHT Coupon Prior to Test Initiation

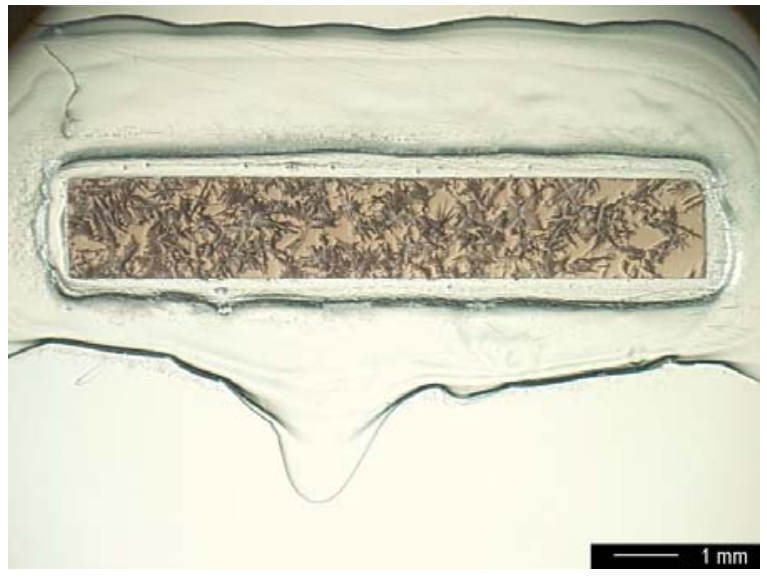

(a)

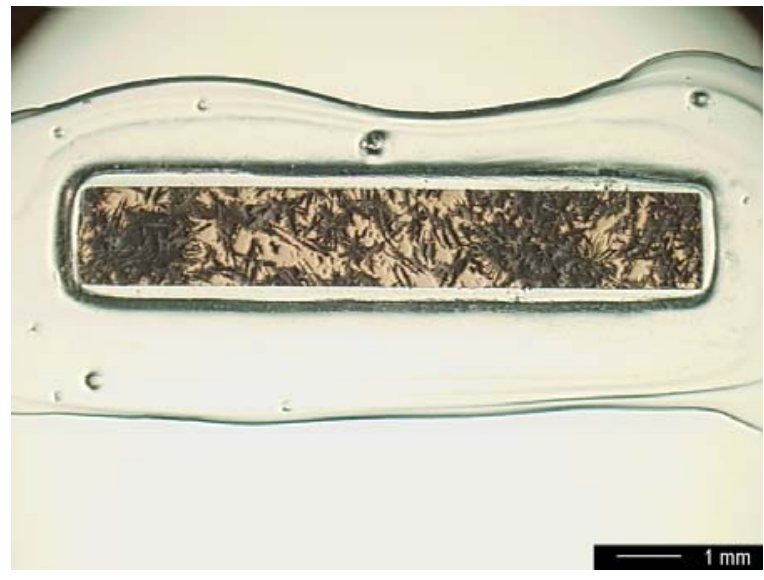

(b)

Figure 4.24. Optical Micrographs of LS-1 VHT Coupon Cross Sections after Testing for (a) 7-days and (b) 14-days

Table 4.7. Measured VHT Responses for LS-1 Specimen

\begin{tabular}{||l|c|c|c||}
\hline Sample ID & $\mathrm{t}($ day $)$ & $\mathrm{m}_{\mathrm{a}}\left(\mathrm{g} / \mathrm{m}^{2}\right)^{*}$ & $\mathrm{r}_{\mathrm{a}}\left(\mathrm{g} / \mathrm{m}^{2} / \mathrm{d}\right)$ \\
\hline LS-1-VHT-007 & 7.0 & -2.6 & -0.4 \\
\hline LS-1-VHT-014 & 13.9 & 10.3 & 0.7 \\
\hline * Estimated measurement uncertainty is $\pm 10 \mathrm{~g} / \mathrm{m}^{2}$. \\
\hline
\end{tabular}




\subsection{Summary and Conclusions}

This test has been designed to provide information for the assessment of the ICV process and equipment performance to support subsequent large-scale and full-scale engineering design. Glass was formulated with 12 mass $\% \mathrm{Na}_{2} \mathrm{O}$. With an extra amount of flux materials added to the top of the dried feed, the final target content of $\mathrm{Na}_{2} \mathrm{O}$ was 10 mass $\%$.

The bulk glass contained a number of crystalline phases- $\mathrm{ZrO}_{2}$ (1.8 mass\%), $\mathrm{CaMgSi}_{2} \mathrm{O}_{6}(2.2 \mathrm{mass} \%$ ), and $\mathrm{ZrSiO}_{4}(0.3$ mass $\%)$. The silica lining separating the container refractory walls from the melt was permeated with molten glass to 15 to $50-\mathrm{mm}$ depth, depending on the position in the block, forming several transition layers. The top surface of the large-scale melt was altered by the collapse of large offgas bubbles from the melting reactions. XRD analysis detected several crystalline phases in the surface glass and the transition areas: silica, albite, hematite, anorthite, baddeleyite, and zircon. A sample from the block bottom contained metallic droplets. Sodium sulfate was identified at the silica-sand-glass interface. Tridymite needles, albite, diopside, and cristobalite were observed by SEM.

"Snow-like" crystalline inclusions were seen in the pre-tested VHT coupon. No visible sign of alteration was observed after testing for 7- and 14-days. The VHT results confirmed that the product was highly durable. 


\subsection{Second AMEC Large-Scale Glass Test (LS-2)}

\subsection{Introduction}

This test has been designed to provide information necessary to assess the ICV process and product quality performance and to support full-scale engineering design. Glass formulation for the LS testing was based on the initial tests described in Section 3.0. This test (LS-2) was conducted with the saltcake simulant, local soil, and chemical additives $\left(\mathrm{B}_{2} \mathrm{O}_{3}\right.$ and $\left.\mathrm{ZrO}_{2}\right)$. Table 5.1 summarizes the amount of dried feed, flux, and starter path materials used in the LS-2 test. Calculated target compositions for LS-2 are shown in Table 5.2.

\subsection{Sample Selection}

The following describes roughly the location of each sample taken from LS-2 glass block (see Figure $5.1)$ :

- GB-01 -- 16 in. $(41 \mathrm{~cm})$ from the bottom of the block and 15 in. $(38 \mathrm{~cm})$ from the south-east corner edge of the melt (including adherent sand layer).

- GB-02 - 24 in. $(61 \mathrm{~cm})$ from south-east corner edge and 22 in. $(56 \mathrm{~cm})$ from the top.

- GB-03 - was at the electrode scar, 10 in. $(25 \mathrm{~cm})$ from the bottom of the east electrode at the south side.

- GB-04 (also known as Rind-01) - was the sand rind sample taken directly below GB-01

- GB-05 - 96 in. $(244 \mathrm{~cm})$ from the east end, 24 in. $(61 \mathrm{~cm})$ from the south wall, at the top of the block, under (and including) the foam.

- GB-06 - southwest wall, at the very top of the block, constituted mostly sand layer in the crown area.

- GB-07 - 18 in. $(46 \mathrm{~cm})$ from north end, 15 in. $(38 \mathrm{~cm})$ from bottom, and 128 in. (325) from west wall, constitutes a sample from roughly the block center.

Five of the seven samples described above (LS-2-GB-01 through -07) were selected to represent the range of possible chemical and physical parameters for the waste form in the LS-2 product. These samples (LS2-GB-01, -02, -03, -05, and -07) ${ }^{\mathrm{a}}$ were tested for chemical composition, oxidation-reduction state of iron, PCT, TCLP, VHT, and the secondary-phase evaluation.

\footnotetext{
a The "-GB" notation in sample ID was omitted in the summary and discussion of the test results in the following sections.
} 
Table 5.1. Amount of Batch Feed, Flux, and Starter Path Materials Used in the LS-2 Test

\begin{tabular}{|c|c|c|}
\hline Type & Material & Mass (kg) \\
\hline \multirow{5}{*}{ Dried Batch feed } & Soil & 5987.7 \\
\hline & Simulant & 2990.5 \\
\hline & $\mathrm{ZrO}_{2}$ & 619.9 \\
\hline & $\mathrm{B}_{2} \mathrm{O}_{3}$ & 433.2 \\
\hline & Subtotal & 10031.3 \\
\hline \multirow{4}{*}{ Flux } & Soil & 2976.2 \\
\hline & $\mathrm{ZrO}_{2}$ & 232.1 \\
\hline & $\mathrm{B}_{2} \mathrm{O}_{3}$ & 166.7 \\
\hline & Subtotal & 3375.0 \\
\hline Starter path & Soil & 185.0 \\
\hline \multicolumn{2}{|l|}{$\begin{array}{r}\text { Starter path } \\
\text { Total }\end{array}$} & 13591.3 \\
\hline
\end{tabular}

Table 5.2. Target Compositions of LS-2 Glass

\begin{tabular}{|c|c|c|}
\hline Component & $\begin{array}{c}\text { Target/ } \\
\text { Batch Feed }^{(\mathrm{a})}\end{array}$ & $\begin{array}{c}\text { Target/ } \\
\text { Combined }^{(\mathbf{b})}\end{array}$ \\
\hline $\mathrm{Al}_{2} \mathrm{O}_{3}$ & 0.0948 & 0.1007 \\
\hline $\mathrm{B}_{2} \mathrm{O}_{3}$ & 0.0524 & 0.0511 \\
\hline $\mathrm{BaO}$ & 0.0005 & 0.0005 \\
\hline $\mathrm{CaO}$ & 0.0286 & 0.0308 \\
\hline $\mathrm{Cl}$ & 0.0015 & 0.0010 \\
\hline $\mathrm{Cr}_{2} \mathrm{O}_{3}$ & 0.0008 & 0.0006 \\
\hline $\mathrm{F}$ & 0.0006 & 0.0004 \\
\hline $\mathrm{Fe}_{2} \mathrm{O}_{3}$ & 0.0444 & 0.0477 \\
\hline $\mathrm{K}_{2} \mathrm{O}$ & 0.0159 & 0.0169 \\
\hline $\mathrm{MgO}$ & 0.0140 & 0.0150 \\
\hline $\mathrm{MnO}$ & 0.0007 & 0.0008 \\
\hline $\mathrm{Na}_{2} \mathrm{O}$ & 0.1670 & 0.1245 \\
\hline $\mathrm{P}_{2} \mathrm{O}_{5}$ & 0.0048 & 0.0039 \\
\hline $\mathrm{SiO}_{2}$ & 0.4836 & 0.5196 \\
\hline $\mathrm{SO}_{3}$ & 0.0069 & 0.0048 \\
\hline $\mathrm{SrO}$ & 0.0003 & 0.0003 \\
\hline $\mathrm{TiO}_{2}$ & 0.0082 & 0.0088 \\
\hline $\mathrm{ZrO}_{2}$ & 0.0751 & 0.0725 \\
\hline Total & 1.0000 & 1.0000 \\
\hline Soil & 0.7081 & 0.7608 \\
\hline Waste & 0.1643 & 0.1156 \\
\hline Additive & 0.1275 & 0.1236 \\
\hline \multicolumn{3}{|c|}{$\begin{array}{l}\text { (a) Calculated assuming only the dried batch feed was } \\
\text { converted to glass. } \\
\text { (b) Calculated assuming that all the flux and starter path } \\
\text { materials were dissolved into glass. }\end{array}$} \\
\hline
\end{tabular}



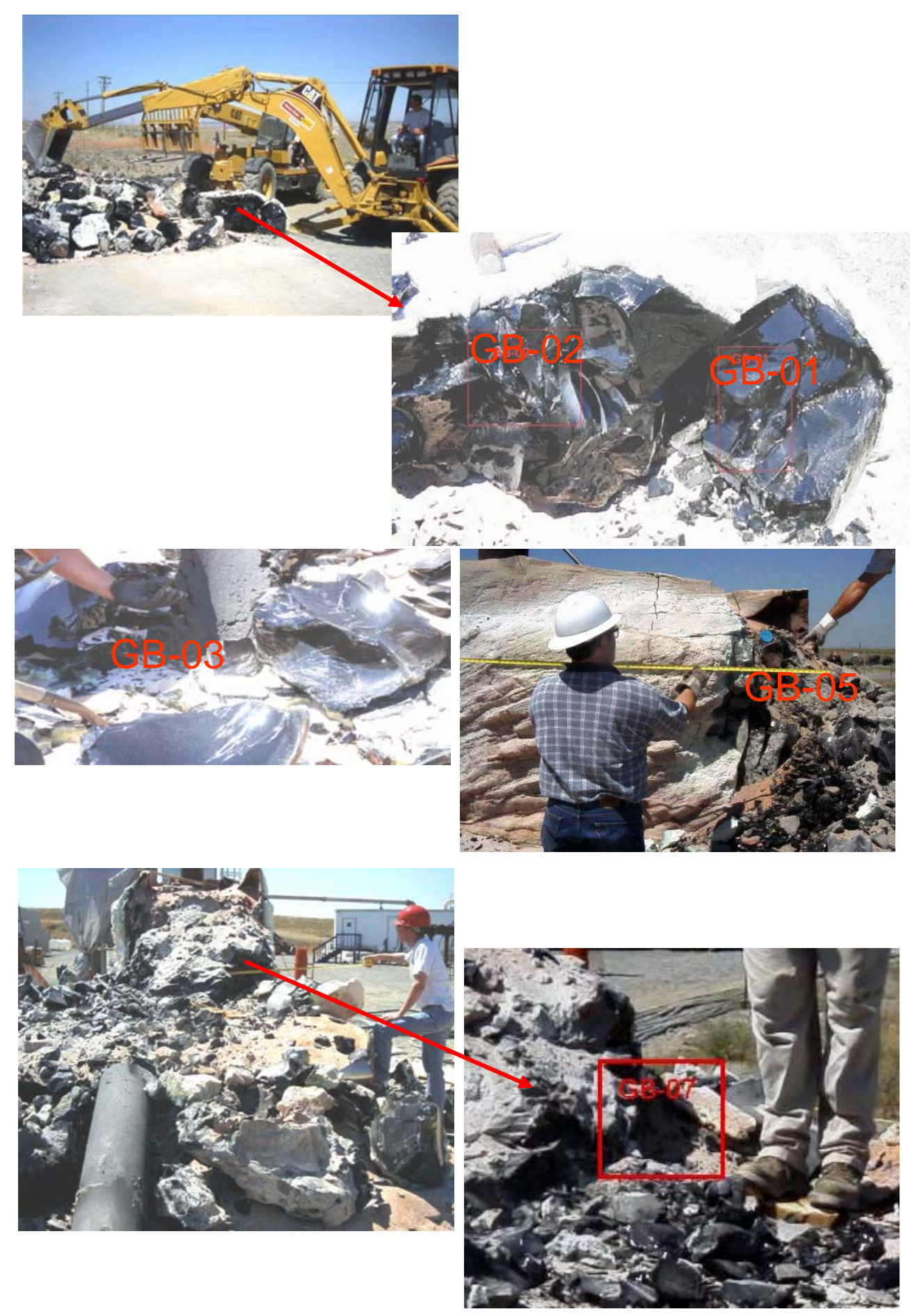

Figure 5.1. Photographs Depicting Sample Locations From LS-2 the Test

\subsection{Chemical Analysis}

The large-scale tests were performed with non-radioactive glass spiked with $\mathrm{Re}_{2} \mathrm{O}_{7}$ as a surrogate for $\mathrm{Tc}_{2} \mathrm{O}_{7}$. Table 5.3 summarizes the results of glass-composition and iron-redox analyses of the five samples 
taken from different locations in the LS-2 glass block. Among the five samples taken from different positions, glass composition was reasonably constant, which indicates that the glass melt was well mixed by convection currents during the melting process. Figure 5.2 shows the comparison of measured and target concentrations of major glass components (those with a target mass fraction higher than 0.03 ) in these samples. The Target/Combined column in Table 5.2 was used for target composition. Measured concentrations were lower than the targets for all components except for $\mathrm{SiO}_{2}$. The difference of measured versus target concentrations ranged from 14 to $19 \%$ for $\mathrm{SiO}_{2}$ and from 4 to $22 \%$ for the remaining six major components. As in engineering-scale tests, the significant increase of $\mathrm{SiO}_{2} \mathrm{resulted}$ from the dissolution of silica sand that lined the container walls to insulate them from the glass melt. The decrease in concentrations of other major oxides was a dilution effect caused by the dissolution of silica in the melt.

The average redox ratio of iron in glass was 0.67 , ranging from 0.38 to 0.80 , which represents a moderately reduced state compared to the highly reduced engineering-scale test glasses. The LS-2-03 glass had an outlying redox value the cause of which is not clear since it was expected to be fully reduced due to its proximity to the graphite electrode.

The least-square-regression approach expressed by the following equation was used to obtain the fraction of sand dissolved in each glass sample:

$$
S=\left(\mathbf{g}^{\mathrm{T}} \mathbf{g}\right)^{-1} \mathbf{g}^{\mathrm{T}} \mathbf{G}
$$

where the $\mathbf{g}$ is a two-column matrix of the target glass composition (the first column) and the sand composition (the second column) and $\mathbf{G}$ is the measured composition vector of glass with dissolved send. Table 5.4 lists the $S$ values obtained from Equation 5.1, using the analyzed compositions of sand and LS2 glass samples in Table 4.1 and Table 5.3. Estimated $S$ ranged from 0.15 to 0.20 with an average of 0.17 . As expected, this estimated value is smaller than those obtained from engineering-scale tests, i.e., 0.30 to 0.39 for ES-1 and 0.18 to 0.23 for ES-2 (the same formula was used to obtain these estimates).

Table 5.3. Measured Compositions of LS-2 Glass Samples (in mass fractions)

\begin{tabular}{||l|c|c|c|c|c|c||}
\hline \hline \multicolumn{1}{|c|}{ Component } & LS-2-01 & LS-2-02 & LS-2-03 & LS-2-05 & LS-2-07 & Average \\
\hline $\mathrm{Al}_{2} \mathrm{O}_{3}$ & 0.0859 & 0.0864 & 0.0851 & 0.0827 & 0.0867 & 0.0854 \\
\hline $\mathrm{B}_{2} \mathrm{O}_{3}$ & 0.0484 & 0.0486 & 0.0474 & 0.0468 & 0.0487 & 0.0480 \\
\hline $\mathrm{CaO}$ & 0.0286 & 0.0293 & 0.0293 & 0.0280 & 0.0296 & 0.0290 \\
\hline $\mathrm{Cr}_{2} \mathrm{O}_{3}$ & 0.0007 & 0.0007 & 0.0008 & 0.0007 & 0.0007 & 0.0007 \\
\hline $\mathrm{Fe}_{2} \mathrm{O}_{3}$ & 0.0440 & 0.0439 & 0.0436 & 0.0428 & 0.0441 & 0.0437 \\
\hline $\mathrm{K}_{2} \mathrm{O}$ & 0.0158 & 0.0157 & 0.0155 & 0.0150 & 0.0166 & 0.0157 \\
\hline $\mathrm{MgO}$ & 0.0130 & 0.0133 & 0.0133 & 0.0128 & 0.0132 & 0.0131 \\
\hline $\mathrm{Na}_{2} \mathrm{O}$ & 0.1019 & 0.1026 & 0.1024 & 0.0981 & 0.1051 & 0.1020 \\
\hline $\mathrm{P}_{2} \mathrm{O}_{5}$ & 0.0032 & 0.0032 & 0.0034 & 0.0033 & 0.0034 & 0.0033 \\
\hline $\mathrm{SiO}_{2}$ & 0.5934 & 0.5926 & 0.6011 & 0.6162 & 0.5925 & 0.5992 \\
\hline $\mathrm{SO}_{3}$ & 0.0001 & 0.0000 & 0.0002 & 0.0002 & 0.0000 & 0.0001 \\
\hline $\mathrm{TiO}_{2}$ & 0.0076 & 0.0078 & 0.0078 & 0.0076 & 0.0078 & 0.0077 \\
\hline $\mathrm{ZrO}$ & 0.0567 & 0.0578 & 0.0581 & 0.0567 & 0.0588 & 0.0576 \\
\hline $\mathrm{Total}^{\mid}$ & 0.9918 & 1.0124 & 0.98 & 0.9854 & 0.9867 & 0.9913 \\
\hline $\mathrm{Redox}^{\mid}$ & 0.75 & 0.62 & 0.38 & 0.78 & 0.80 & 0.67 \\
\hline $\mathrm{Fe}(\mathrm{II}) / \mathrm{Fe}$ (total) & 0.78 \\
\hline
\end{tabular}




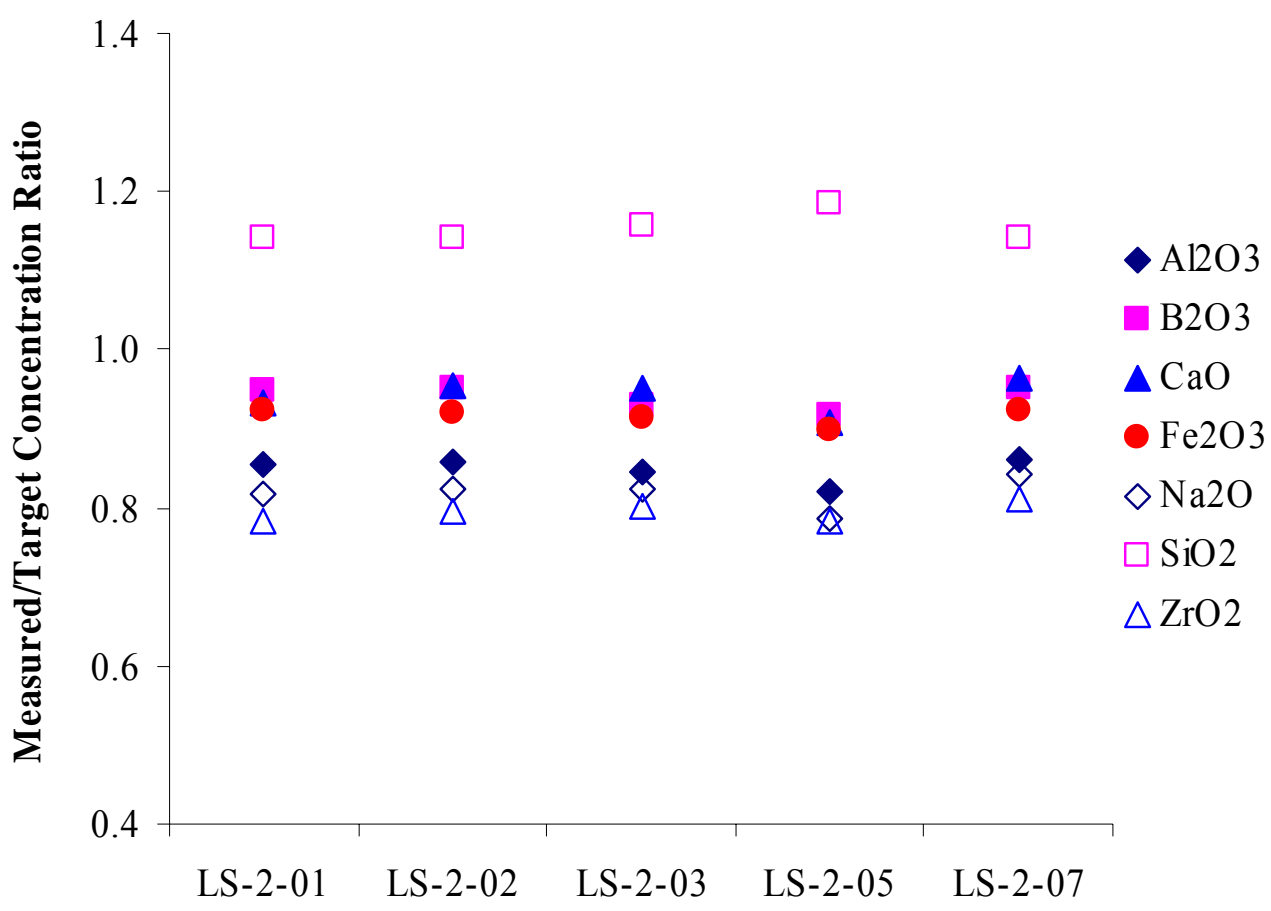

LS-2 Sample ID

Figure 5.2. Ratio of Measured and Target (Adjusted) Concentrations Selected Major Components in LS-2 Glasses

Table 5.4. Estimated Mass Fraction of Sand Dissolved in the LS-2 Glass Samples $(\mathcal{S})$ Calculated Based on Analyzed Glass Compositions

\begin{tabular}{||c|c|c|c|c|c|c||}
\hline \hline Component & LS-2-01 & LS-2-02 & LS-2-03 & LS-2-05 & LS-2-07 & Average \\
\hline$S$ & 0.16 & 0.16 & 0.17 & 0.20 & 0.15 & 0.17 \\
\hline \hline
\end{tabular}

\subsection{Product Consistency Test}

Table 5.5 summarizes the PCT response of LS-2 glass samples. The normalized releases were calculated based on the "Combined" target compositions in Table 5.2. The normalized releases from LS-2 glasses were much lower (roughly 1/4) than both quenched and SC-treated ASCM-02 glasses of similar target compositions. This low PCT release can be attributed to the dissolution of silica sand and the use of the sodium-free flux materials on top of the LS-2 feed. There was little difference between samples taken at different locations suggesting that the glass was well homogenized during the melting process. The same conclusion was reached from the analyses of measured glass compositions (see Section 5.3). 
Table 5.5. PCT Responses for LS-2 Glasses

\begin{tabular}{|c|c|c|c|c|c|}
\hline Component & LS-2-01 & LS-2-02 & LS-2-03 & LS-2-05 & LS-2-07 \\
\hline \multicolumn{6}{|c|}{ 7-day PCT leachate concentration $(\mathrm{mg} / \mathrm{L})$} \\
\hline$c_{N a}$ & 14.1 & 14.0 & 13.8 & 14.3 & 14.5 \\
\hline$c_{A l}$ & 3.78 & 3.57 & 3.55 & 3.76 & 3.74 \\
\hline$C_{B}$ & 3.72 & 3.13 & 3.15 & 3.13 & 3.03 \\
\hline$c_{S i}$ & 26.6 & 27.9 & 26.4 & 30.2 & 28.6 \\
\hline \multicolumn{6}{|c|}{ 7-day PCT Normalized release $\left(\mathrm{g} / \mathrm{m}^{2}\right)$} \\
\hline$r_{N a}$ & 0.076 & 0.076 & 0.075 & 0.077 & 0.078 \\
\hline$r_{A l}$ & 0.035 & 0.034 & 0.033 & 0.035 & 0.035 \\
\hline$r_{B}$ & 0.117 & 0.099 & 0.099 & 0.099 & 0.096 \\
\hline$r_{S i}$ & 0.055 & 0.057 & 0.054 & 0.062 & 0.059 \\
\hline
\end{tabular}

\subsection{Vapor Hydration Test}

Five samples (LS-2- 01, -02, -03, -05, and -07) were tested for 14-days at $200^{\circ} \mathrm{C}$. Two specimens of LS2-05 were tested since this sample included some of the foam-like material and was more heterogeneous than the other samples. The VHT results are listed in Table 5.6. Within experimental uncertainty, the 14day VHT responses of all these specimens are zero, suggesting a very durable glass product.

Table 5.6. Measured VHT Responses for LS-2 Specimens

\begin{tabular}{||l|c|c|c||}
\hline \hline Sample ID & $\mathrm{t}$ (day) & $\mathrm{m}_{\mathrm{a}}\left(\mathrm{g} / \mathrm{m}^{2}\right)^{*}$ & $\mathrm{r}_{\mathrm{a}}\left(\mathrm{g} / \mathrm{m}^{2} / \mathrm{d}\right)$ \\
\hline LS-2-01-VHT-014 & 14.0 & 2.5 & 0.2 \\
\hline LS-2-02-VHT-014 & 14.0 & -1.3 & -0.1 \\
\hline LS-2-03-VHT-014 & 14.0 & -3.8 & -0.3 \\
\hline LS-2-05(1)-VHT-014 & 14.0 & 5.0 & 0.4 \\
\hline LS-2-05(2)-VHT-014 & 14.0 & 1.3 & 0.1 \\
\hline LS-2-07-VHT-014 & 14.0 & -1.3 & -0.1 \\
\hline Average & 14.0 & 0.4 & 0.0 \\
\hline * Estimated measurement uncertainty is $\pm 10 \mathrm{~g} / \mathrm{m}^{2}$. \\
\hline
\end{tabular}

\subsection{Toxicity Characteristic Leach Procedure}

Table 5.7 lists the TCLP responses of the glass samples taken from different positions of the LS-2 glass block. Table 5.7 shows that the Cr releases are nearly an order of magnitude lower than the Universal Treatment Standard (UTS) limit of $0.6 \mathrm{mg} / \mathrm{L}$. The LS-2 glasses had slightly lower TCLP $r_{\mathrm{B}}$ compared to quenched and SC-treated ASCM-02 glasses, which is consistent with the PCT results although the difference is smaller than in PCT results. 
Table 5.7. TCLP Responses for LS-2 Glasses

\begin{tabular}{||c|c|c|c|c|c||}
\hline $\begin{array}{c}\text { Glass } \\
\text { Sample }\end{array}$ & LS-2-01 & LS-2-02 & LS-2-03 & LS-2-05 & LS-2-07 \\
\hline$C_{B}(\mathrm{mg} / \mathrm{L})$ & 0.37 & 0.30 & 0.30 & 0.33 & 0.29 \\
\hline$C_{C r}(\mathrm{mg} / \mathrm{L})$ & 0.0051 & & & & 0.0068 \\
\hline$r_{B}(\mathrm{mg} / \mathrm{L})$ & 23.33 & 18.92 & 18.92 & 20.81 & 18.28 \\
\hline $\begin{array}{l}\text { The italicized values in highlighted cells are estimated results because they are below the reporting } \\
\text { limits }(0.25 \mathrm{mg} / \mathrm{L} \text { for Cr and 0.5 mg/L for B). }\end{array}$
\end{tabular}

\subsection{Glass Density}

Glass density was measured for the same set of samples characterized in previous tests (GB-01, $-02,-03$, $05,-06$, and -07). The results are listed in Table 5.8. The density values range from roughly 2.56 to 2.59 $\mathrm{g} / \mathrm{mL}$ with a mean of $2.58 \mathrm{~g} / \mathrm{mL}$ and a standard deviation of $0.01 \mathrm{~g} / \mathrm{mL}$.

Table 5.8. Measured Densities of LS-2 Glass Samples

\begin{tabular}{||l|c|c||}
\hline \hline Sample ID & Mean Density $(\mathrm{g} / \mathrm{mL})$ & Standard Deviation $(\mathrm{g} / \mathrm{mL})$ \\
\hline LS-2-01-D & 2.5927 & 0.0004 \\
\hline LS-2-02-D & 2.5892 & 0.0003 \\
\hline LS-2-03-D & 2.5878 & 0.0001 \\
\hline LS-2-05-D & 2.5847 & 0.0004 \\
\hline LS-2-06-D & 2.5611 & 0.0007 \\
\hline LS-2-07-D & 2.5881 & 0.0003 \\
\hline Average & 2.5839 & 0.0115 \\
\hline
\end{tabular}

\subsection{Secondary Phase Identification}

Samples of glass (LS-2-01 to -07) were analyzed for the amount and type of inclusions, such as crystalline material, metallic droplets, graphite, or bubbles that formed during the process or cooling. Glasses LS-2-01, LS-2-02, LS-2-07 did not contain measurable secondary phases. Results of SEM-EDS and XRD analysis of LS-2-03 and LS-2-05 glasses are shown in Figure 5.3 to Figure 5.8 and in Table 5.9. Figure 5.3 and Figure 5.4 show SEM micrographs of zircon $\left(\mathrm{ZrSiO}_{4}\right)$ crystals and indicate that these zircon crystals nucleate and grow from dissolving zirconia as it reacts with silica in the glass melt. Residual grains of silica were converted to tridymite $\left(\mathrm{SiO}_{2}\right)$ as shown in Figure 5.5. As the EDS dot map in Figure 5.6 shows, the amorphous phase contains more silica than zircon crystals. The residual zirconia, some of which recrystallized, probably during cooling, to baddeleyite, is clearly identifiable by the absence of $\mathrm{Si}$. Interestingly, the zircon crystals contain chromium. Diopside $\left(\mathrm{CaMg}_{0.5} \mathrm{Fe}_{0.5} \mathrm{Si}_{2} \mathrm{O}_{6}\right)$ has also been identified, see Figure 5.7. Also in Figure 5.7, an irregular shape of iron-rich material is evidenced, probably a residue from the soil. Table 5.9 lists crystallinity fractions in samples LS-2-03 and LS-2-05. Only zircon was found in a measurable fraction ( 0.35 mass $\%)$ in sample LS-2-03. Apart from zircon (2 mass $\%$ ), several silica phases (quartz, tridymite, and cristobalite, 2 mass $\%$ altogether) occurred in sample LS-2-05. 


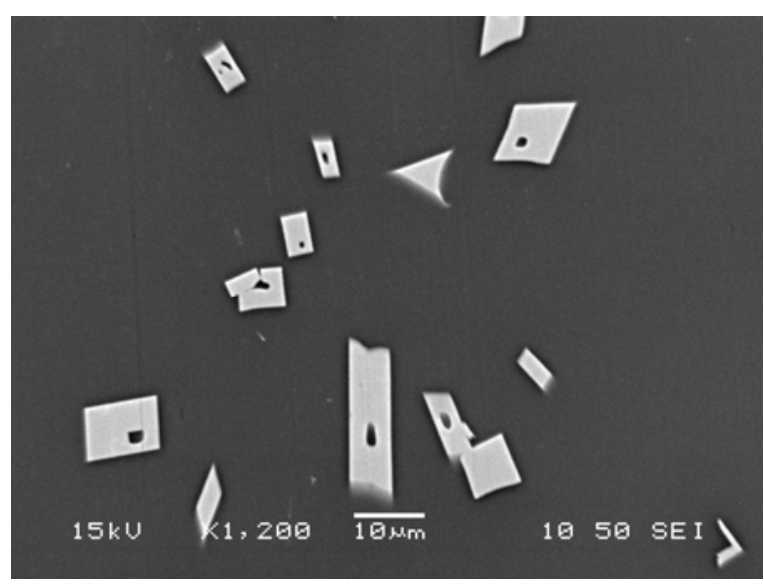

$\mathrm{a}$

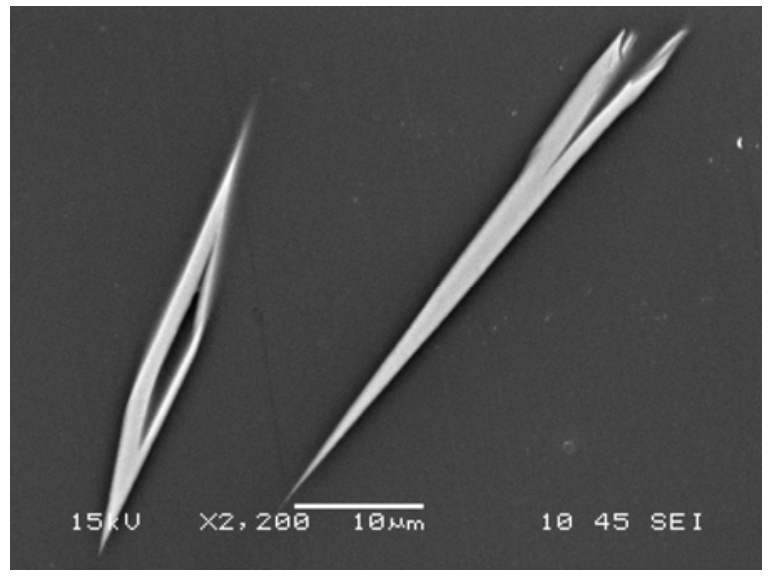

c

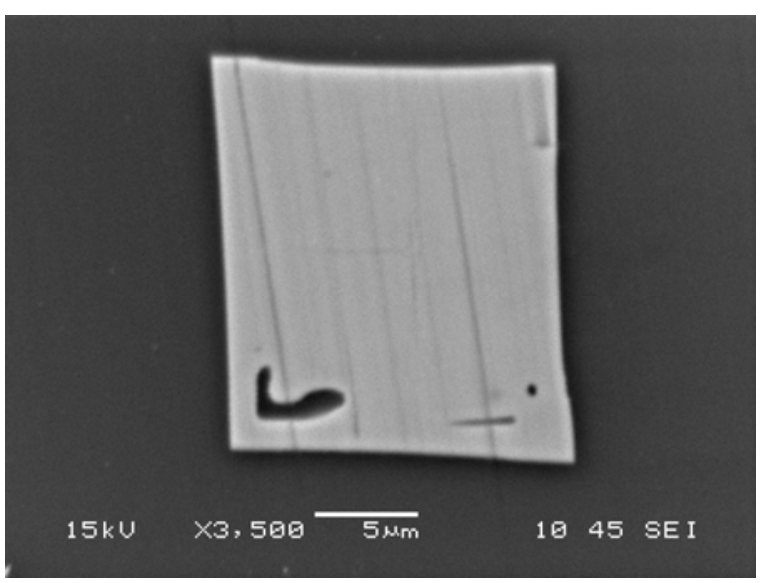

b

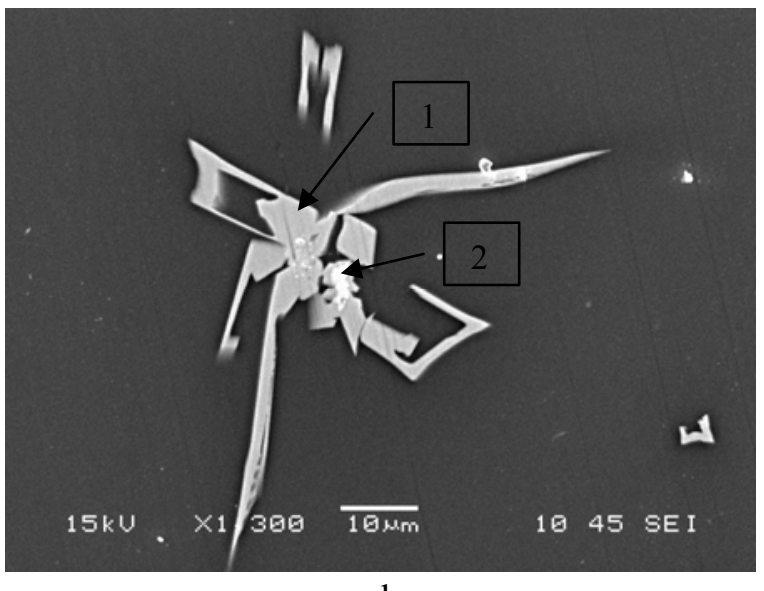

d

Figure 5.3. SEM Micrographs of Crystals in Sample LS-2-03-200-014: Individual Crystals of Zircon (a to c) and Zircon Crystals ( 1 in d) Nucleated on and Dissolving Zirconia (2 in d) 


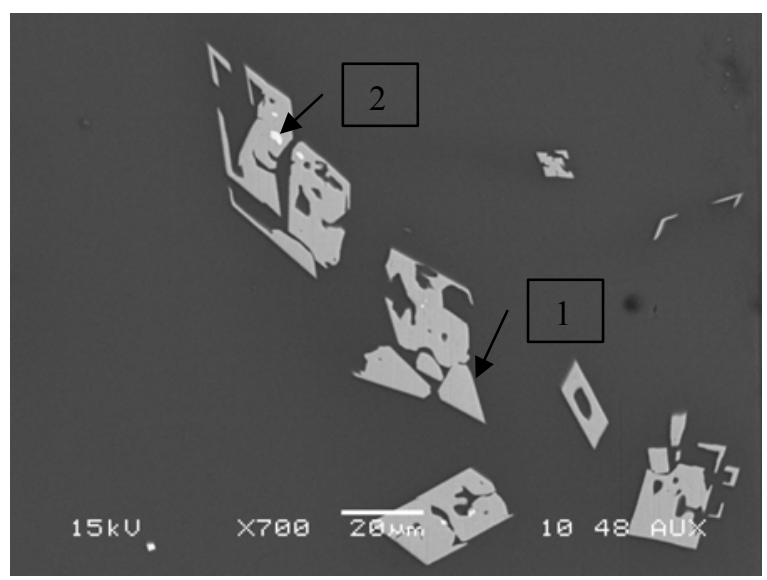

a

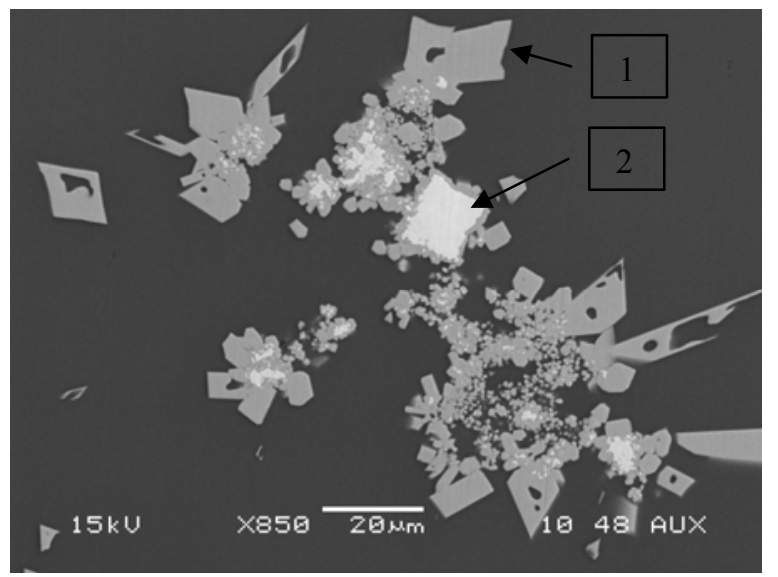

c

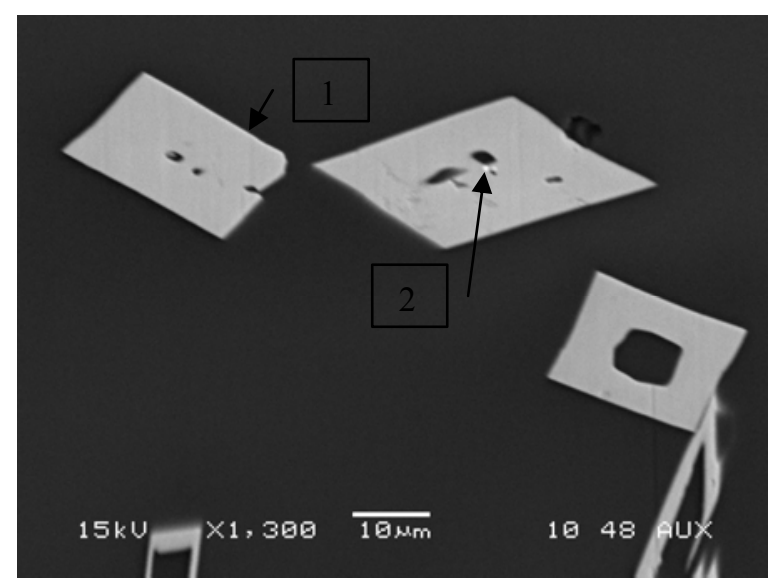

b

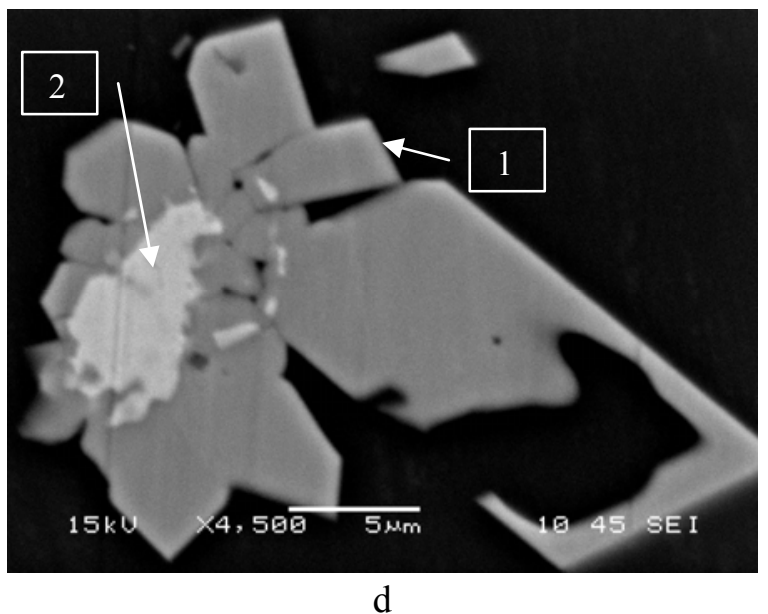

Figure 5.4. SEM Micrographs of Crystals in Sample LS-2-05(1)-200-014: Zircon Crystals (1) with Residual Zirconia (2) 


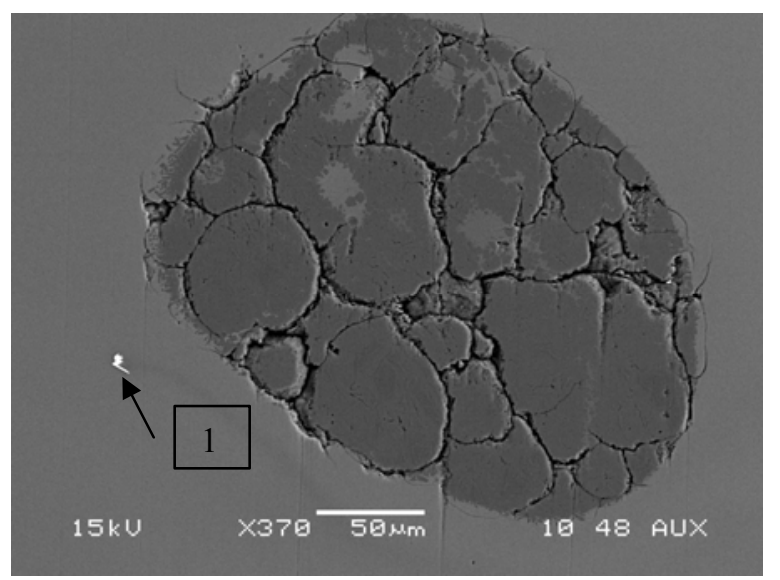

a

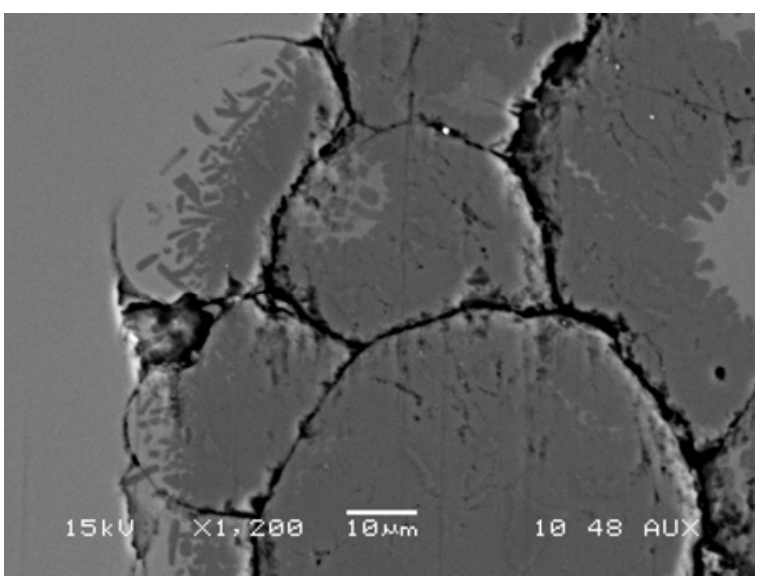

b

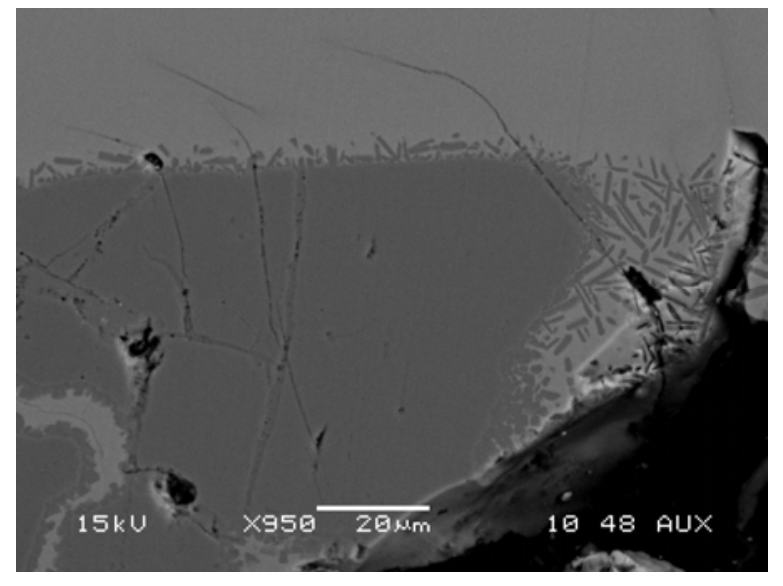

c

Figure 5.5. SEM Micrographs of Crystals in Sample LS-2-05(1)-200-014: Tridymite Crystals Originated from Residual Sand Grains; a Tiny Crystal of Zircon (1) is Seen in (a) 


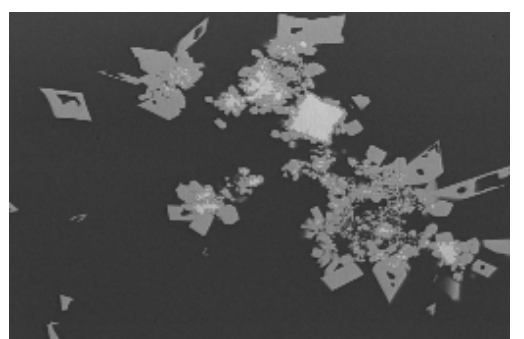

LS-2-05(01)-200-014

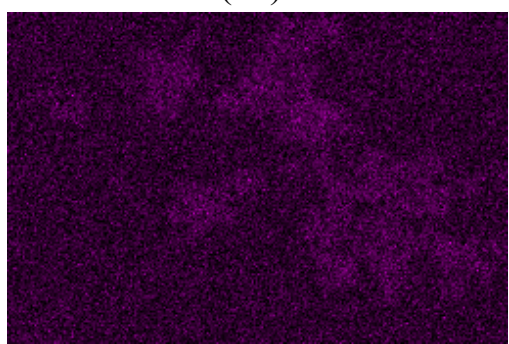

$\mathrm{Cr}$

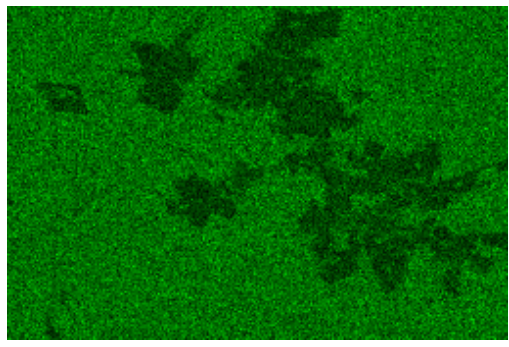

K

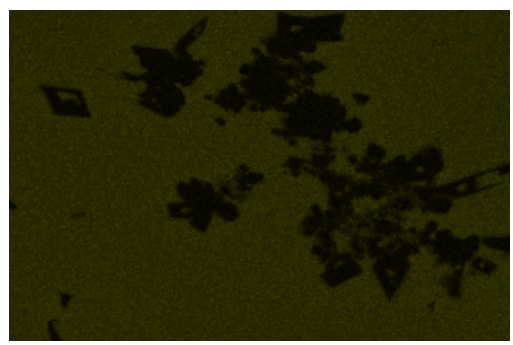

$\mathrm{Al}$

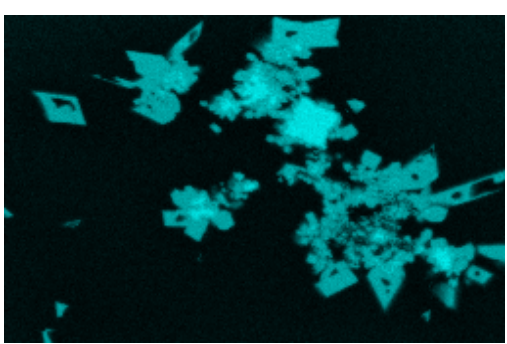

$\mathrm{Zr}$

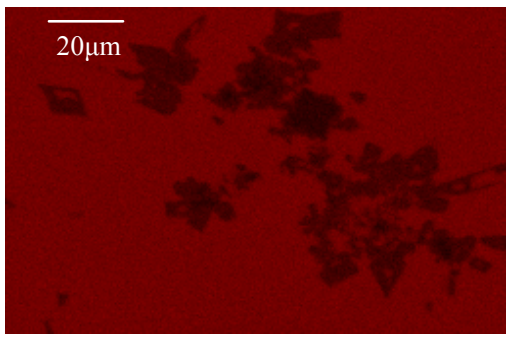

$\mathrm{O}$

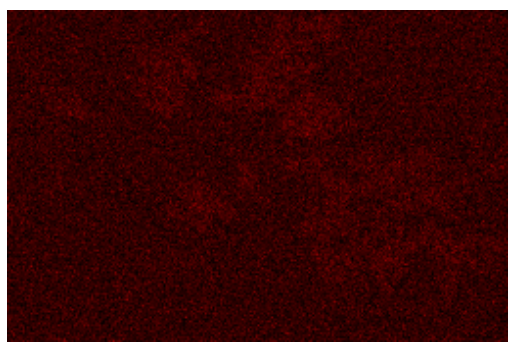

$\mathrm{Cl}$

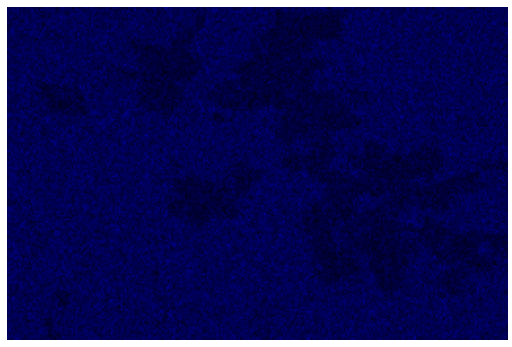

$\mathrm{Mg}$

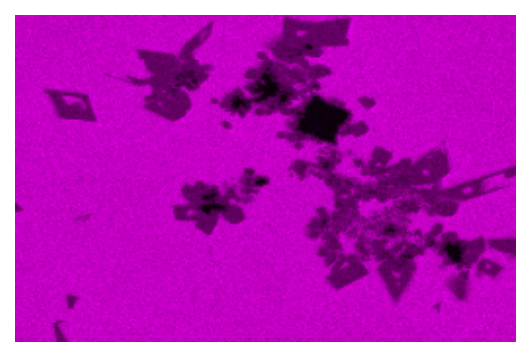

$\mathrm{Si}$

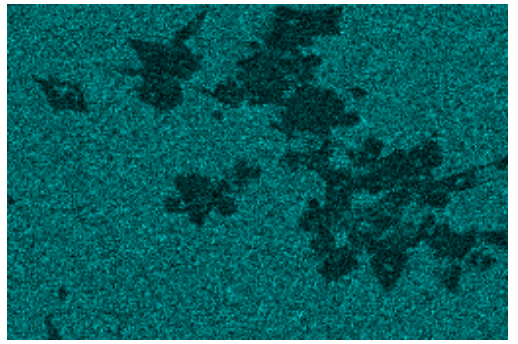

$\mathrm{Fe}$

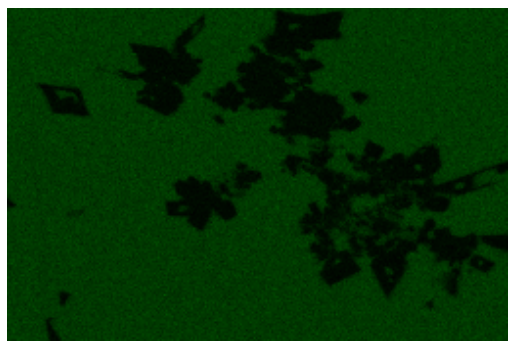

$\mathrm{Na}$

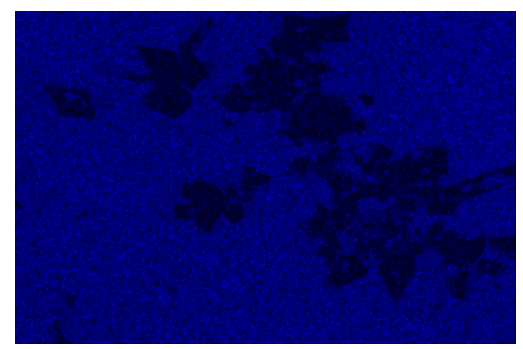

$\mathrm{Ca}$

Figure 5.6. EDS Dot Map of a Cluster of Zircon Crystals Growing from Dissolving Zirconia in Sample LS-2-05(01)-200-014 


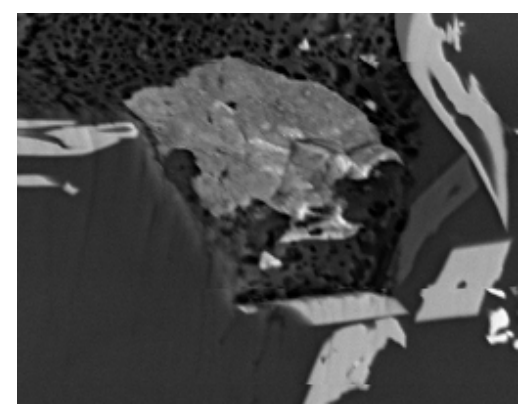

LS-2-05(01)-200-014

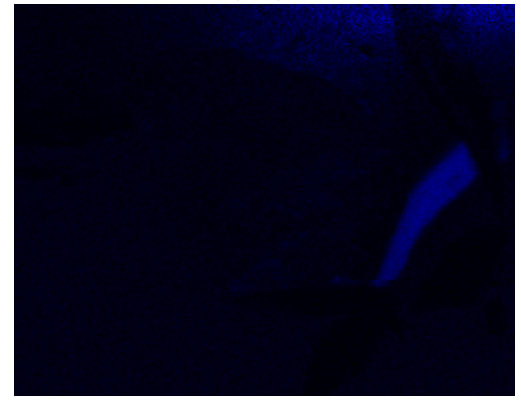

$\mathrm{Ca}$

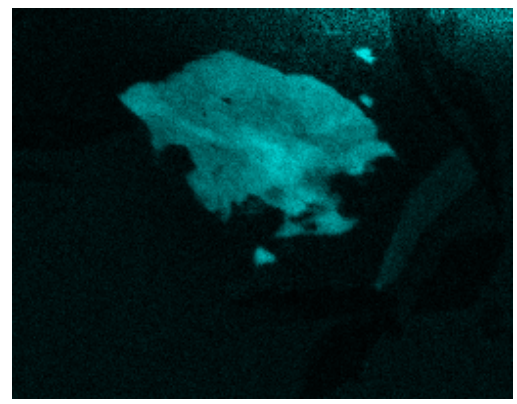

$\mathrm{Fe}$

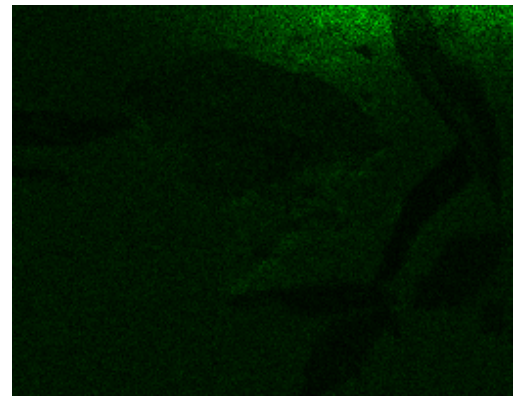

K

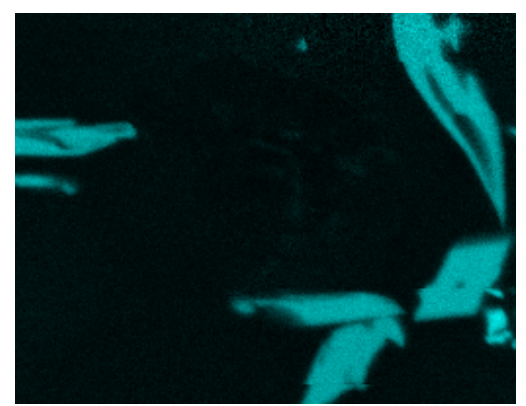

$\mathrm{Zr}$

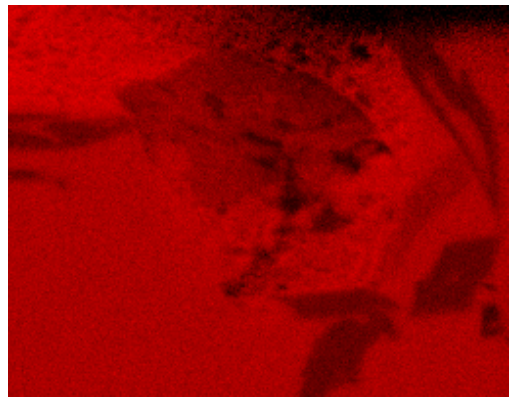

$\mathrm{O}$

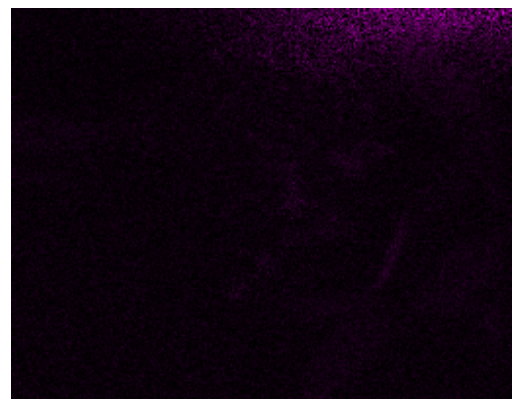

$\mathrm{Cr}$

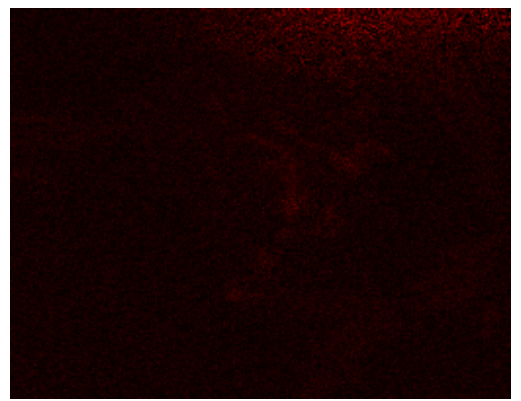

$\mathrm{Cl}$

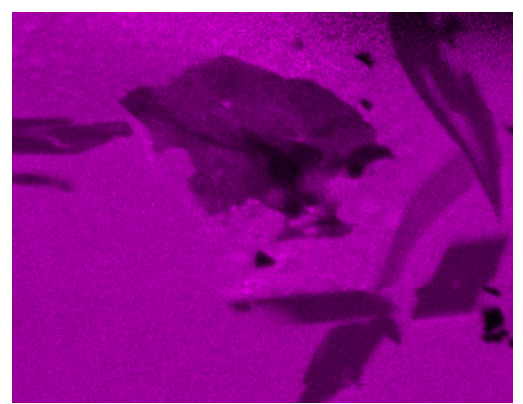

$\mathrm{Si}$

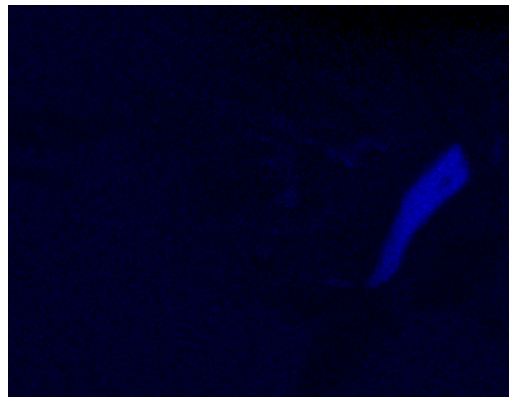

$\mathrm{Mg}$

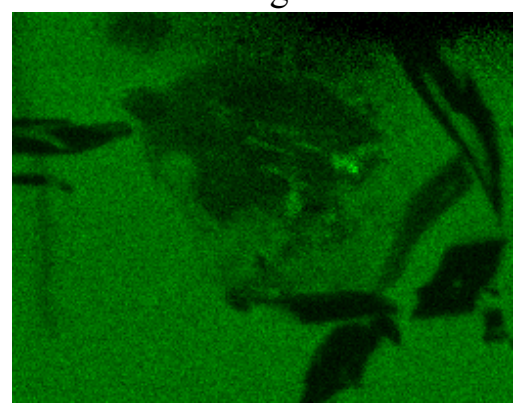

$\mathrm{Na}$

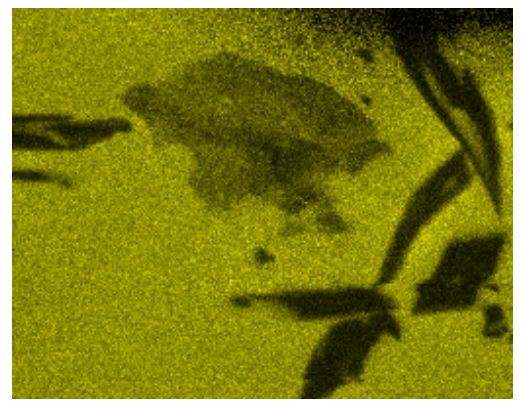

$\mathrm{Al}$

Figure 5.7. EDS Dot Map an Iron-Rich Inclusion, a Diopside Crystal, and Zircon Crystals in Sample LS-2-05(01)-200-014 

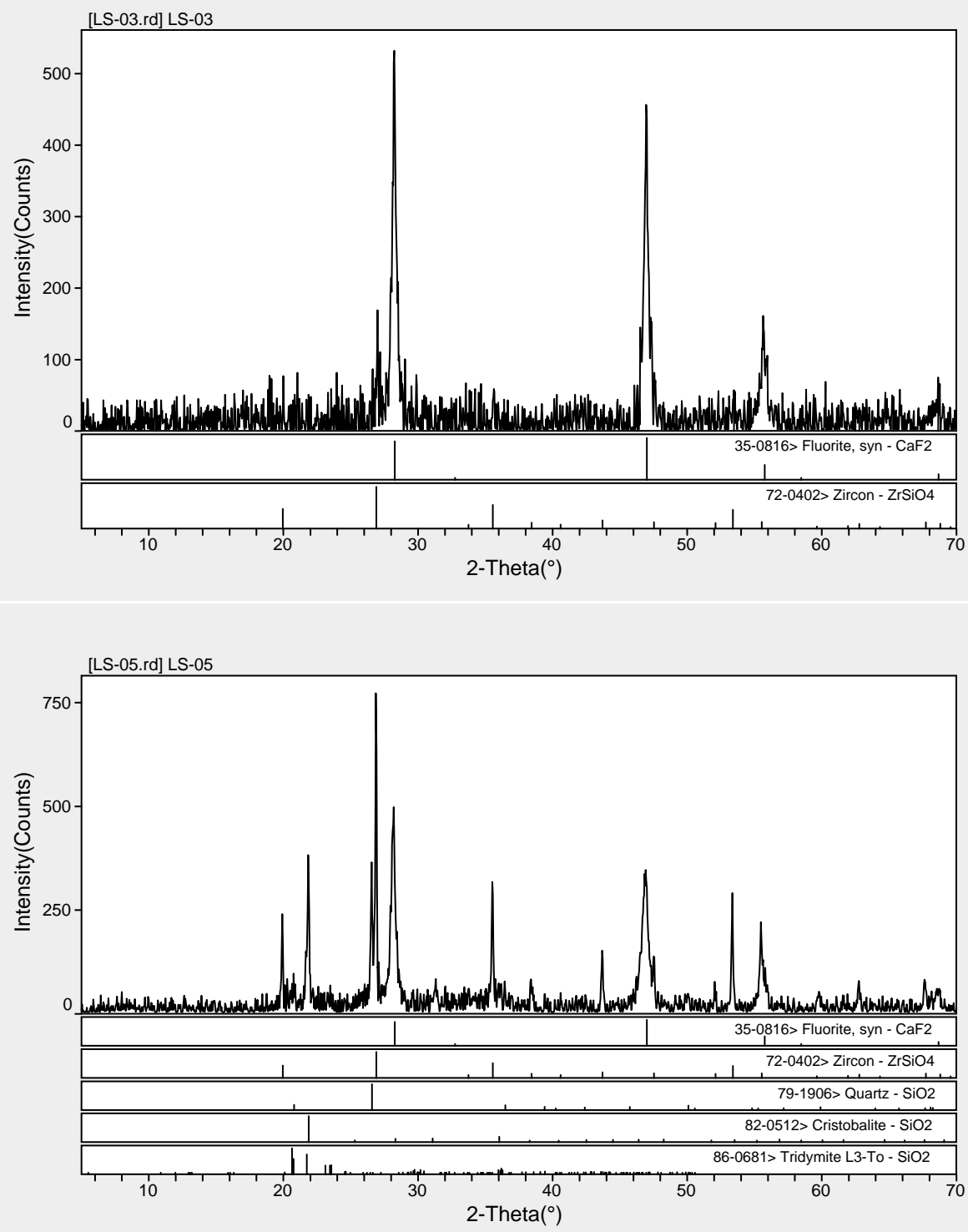

Figure 5.8. XRD Pattern of Samples LS-2-03 and LS-2-05

Table 5.9. Crystallinity Content in Samples LS-2-03 and LS-2-05 by XRD

\begin{tabular}{||c|c|c|c|c||}
\hline Glass ID & $\begin{array}{c}\mathbf{Z r S i O}_{4} \\
(\mathrm{mass} \%)\end{array}$ & $\begin{array}{c}\text { Quartz } \\
(\text { mass\%) }\end{array}$ & $\begin{array}{c}\text { Tridymite } \\
(\text { mass\%) }\end{array}$ & $\begin{array}{c}\text { Cristobalite } \\
\text { (mass\%) }\end{array}$ \\
\hline LS-2-03 & 0.35 & 0 & 0 & 0 \\
\hline LS-2-05 & 2.14 & 0.87 & 0.32 & 0.80 \\
\hline
\end{tabular}




\subsection{Summary and Conclusions}

This test has been designed to provide information necessary to assess the ICV process and product quality performance and to support full-scale engineering design. Glass was formulated with 16.8 mass $\%$ $\mathrm{Na}_{2} \mathrm{O}$. An extra amount of flux materials was added to the top of the dried feed. Assuming perfect mixing, the final target content of $\mathrm{Na}_{2} \mathrm{O}$ was 12.8 mass\%. Among five samples taken from different positions of the final block, glass composition was reasonably constant, indicating that the glass melt was well mixed. Due to the dissolution of silica sand that lined the container walls, measured concentrations of all components except $\mathrm{SiO}_{2}$ were lower than the targets. The average analyzed $\mathrm{Na}_{2} \mathrm{O}$ content in glass dropped to 10.2 mass $\%$. The average redox ratio of iron in glass was 0.67 , ranging from 0.38 to 0.80 .

The average normalized 7-day PCT Na release from LS-2 glasses was $0.08 \mathrm{~g} / \mathrm{m}^{2}$. The average TCLP Cr releases was $0.006 \mathrm{mg} / \mathrm{L}$. Both PCT and TCLP releases are more than an order of magnitude lower than the regulatory limits. The 14-day VHT responses of all specimens tested were zero, suggesting a very durable glass product. Glass density ranged from 2.56 to $2.59 \mathrm{~g} / \mathrm{mL}$ with a mean of $2.58 \mathrm{~g} / \mathrm{mL}$ and a standard deviation of $0.01 \mathrm{~g} / \mathrm{mL}$. SEM-EDS and XRD analysis of glass samples detected a small fraction of zircon, tridymite $\left(\mathrm{SiO}_{2}\right)$, baddeleyite, and diopside phases only in two of five samples analyzed. 


\subsection{Third AMEC Large-Scale Test (LS-3)}

\subsection{Introduction}

The purpose of LS-3 was to demonstrate the ICV process with the baseline glass composition, including $20 \% \mathrm{Na}_{2} \mathrm{O}$. The component masses used in LS-3 are listed in Table 6.1 and the resulting estimated compositions are listed in Table 6.2.

Table 6.1. Amount of Batch Feed, Flux, and Starter Path Materials Used in LS-3 Test

\begin{tabular}{||c|c|c||}
\hline Type & Material & Mass (kg) \\
\hline \multirow{4}{*}{ Dried Batch feed } & Soil & 5770.8 \\
\cline { 2 - 3 } & Simulant & 3687.3 \\
\cline { 2 - 3 } & $\mathrm{ZrO}_{2}$ & 652.9 \\
\cline { 2 - 3 } & $\mathrm{B}_{2} \mathrm{O}_{3}$ & 456.4 \\
\cline { 2 - 3 } & $\mathrm{Subtotal}_{1}$ & 10567.4 \\
\hline \multirow{4}{*}{ Flux } & Soil & 1168.0 \\
\cline { 2 - 3 } & $\mathrm{ZrO}_{2}$ & 106.1 \\
\cline { 2 - 3 } & $\mathrm{B}_{2} \mathrm{O}_{3}$ & 75.9 \\
\cline { 2 - 3 } & $\mathrm{Subtotal}_{1}$ & 1350.0 \\
\hline Starter path & Soil & 185.0 \\
\hline Total & & 12102.4 \\
\hline \hline \multicolumn{2}{|c|}{} &
\end{tabular}

\subsection{Sample Selection}

Figure 6.1 shows a picture of the LS-3 glass block. Seven samples were taken from three breaks roughly perpendicular to the long axis of the block (labeled LS-3-GB-01 through -07). A diagram of the LS-3 block with precise sample locations is shown in Figure 6.2. Photographs of the sample locations are given in Figure 6.3. These samples were taken specifically to span the full range of possible product behavior within the glass block. Five of these seven samples (LS-3-GB-02, -04, -05, -06, and -07) ${ }^{\mathrm{a}}$ were tested for chemical composition, oxidation-reduction state of iron, PCT, TCLP, VHT, and the secondaryphase evaluation. Density was measured for all seven samples.

${ }^{a}$ The "-GB" notation in sample ID was omitted in the summary and discussion of the test results in following sections. 
Table 6.2. Target Compositions of LS-3 Glass

\begin{tabular}{|c|c|c|}
\hline Component & $\begin{array}{c}\text { Target/ } \\
\text { Batch Feed }^{(a)}\end{array}$ & $\begin{array}{c}\text { Target/ } \\
\text { Combined }^{(b)}\end{array}$ \\
\hline $\mathrm{Al}_{2} \mathrm{O}_{3}$ & 0.0904 & 0.0939 \\
\hline $\mathrm{B}_{2} \mathrm{O}_{3}$ & 0.0542 & 0.0536 \\
\hline $\mathrm{BaO}$ & 0.0005 & 0.0005 \\
\hline $\mathrm{CaO}$ & 0.0271 & 0.0283 \\
\hline $\mathrm{Cl}$ & 0.0018 & 0.0015 \\
\hline $\mathrm{Cr}_{2} \mathrm{O}_{3}$ & 0.0010 & 0.0008 \\
\hline $\mathrm{F}$ & 0.0007 & 0.0006 \\
\hline $\mathrm{Fe}_{2} \mathrm{O}_{3}$ & 0.0420 & 0.0440 \\
\hline $\mathrm{K}_{2} \mathrm{O}$ & 0.0152 & 0.0158 \\
\hline $\mathrm{MgO}$ & 0.0132 & 0.0138 \\
\hline $\mathrm{MnO}$ & 0.0007 & 0.0007 \\
\hline $\mathrm{Na}_{2} \mathrm{O}$ & 0.1968 & 0.1706 \\
\hline $\mathrm{P}_{2} \mathrm{O}_{5}$ & 0.0054 & 0.0049 \\
\hline $\mathrm{SiO}_{2}$ & 0.4572 & 0.4789 \\
\hline $\mathrm{SO}_{3}$ & 0.0083 & 0.0070 \\
\hline $\mathrm{SrO}$ & 0.0003 & 0.0003 \\
\hline $\mathrm{TiO}_{2}$ & 0.0078 & 0.0081 \\
\hline $\mathrm{ZrO}_{2}$ & 0.0775 & 0.0765 \\
\hline Total & 1.0000 & 1.0000 \\
\hline Soil & 0.6694 & 0.7012 \\
\hline Waste & 0.1988 & 0.1687 \\
\hline Additive & 0.1318 & 0.1301 \\
\hline \multicolumn{3}{|c|}{$\begin{array}{l}\text { (a) Calculated assuming only the dried batch feed was } \\
\text { converted into glass. } \\
\text { (b) Calculated assuming that all the flux and starter path } \\
\text { materials were dissolved in glass. }\end{array}$} \\
\hline
\end{tabular}

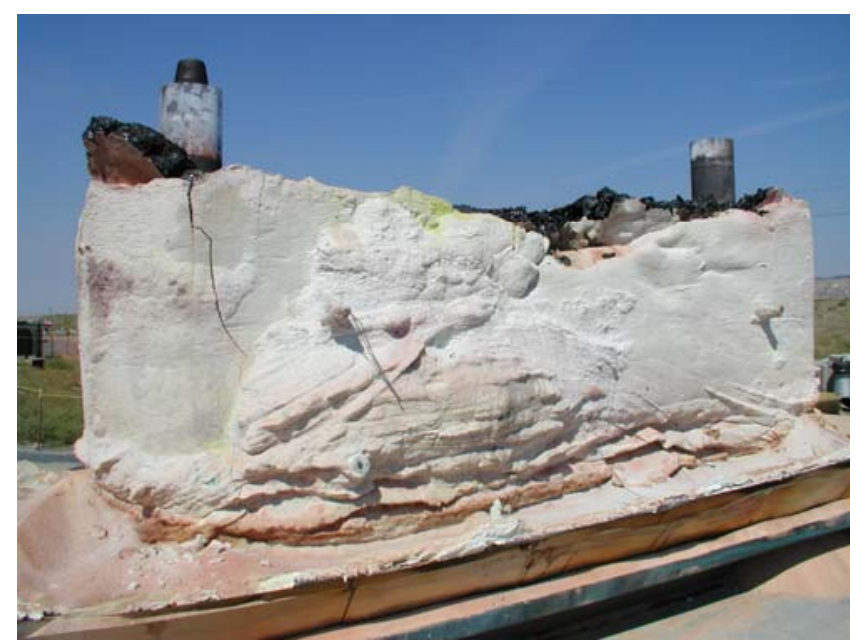

Figure 6.1. Photograph of LS-3 Glass Block 


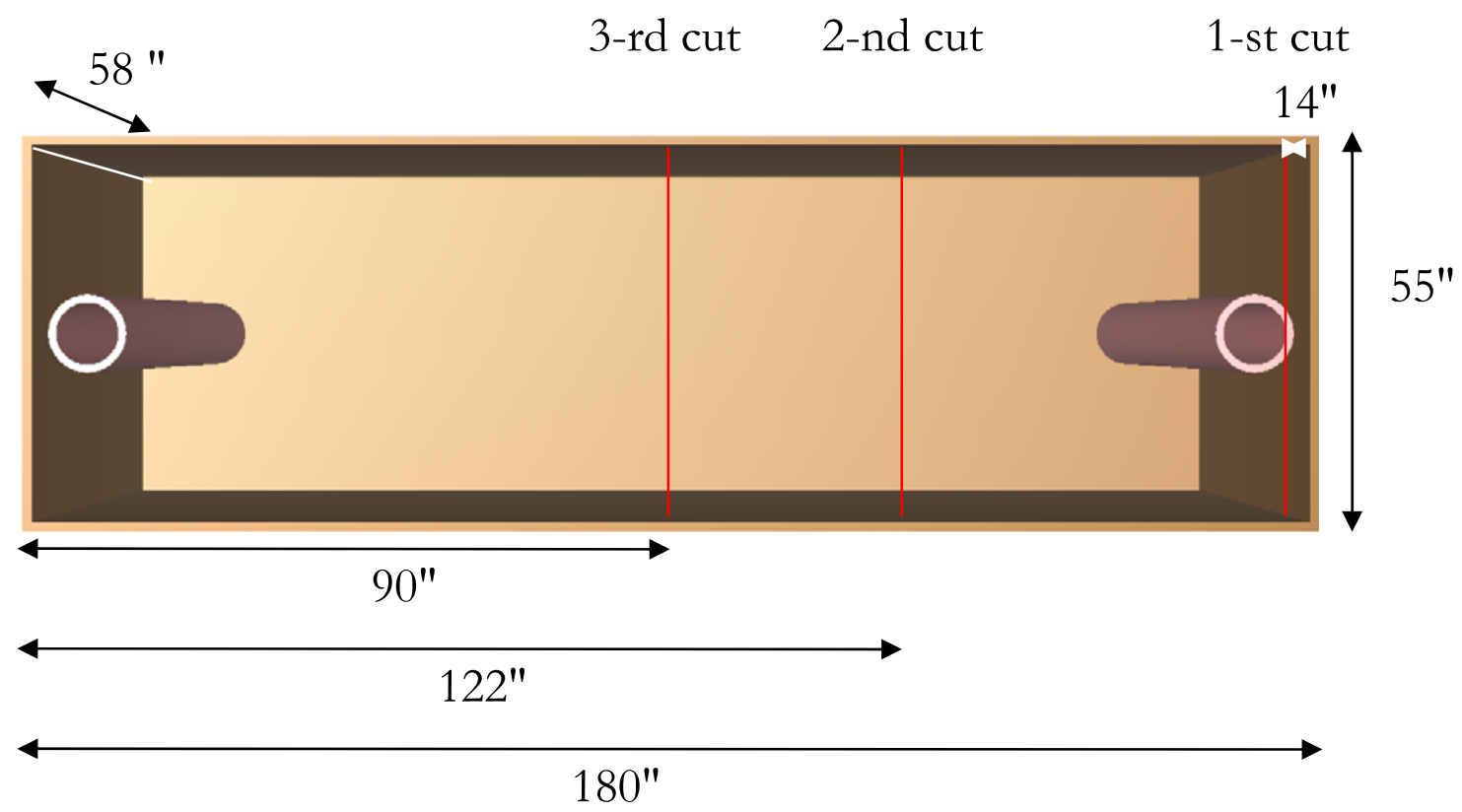

(a)
1-st cut (14")
2-nd cut (122")
3-rd cut (90")

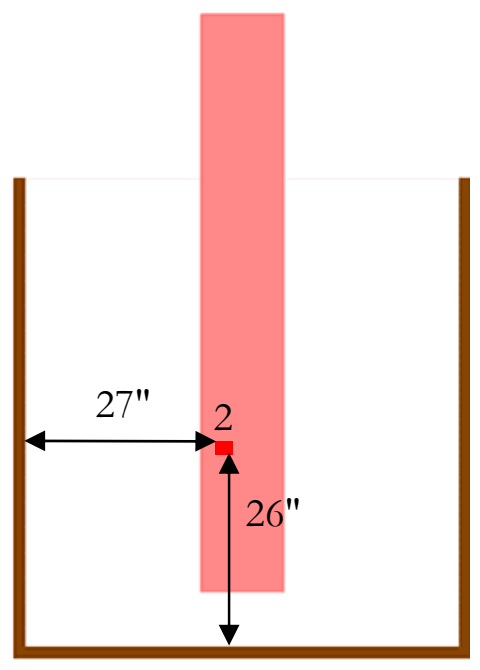

LS-3-GB-01: glass block side LS-3-GB-02: near electrode

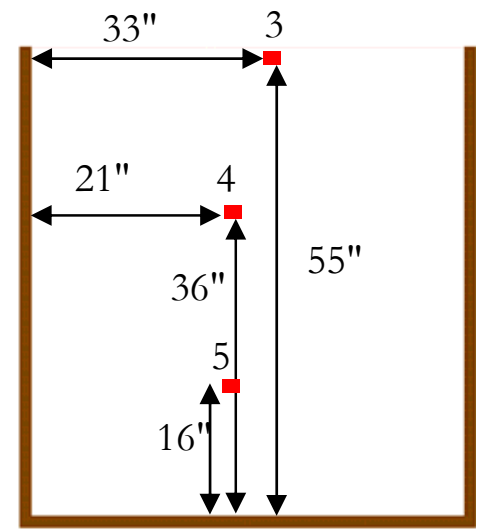

LS-3-GB-03: top layer LS-3-GB-04: near center LS-3-GB-05: near bottom

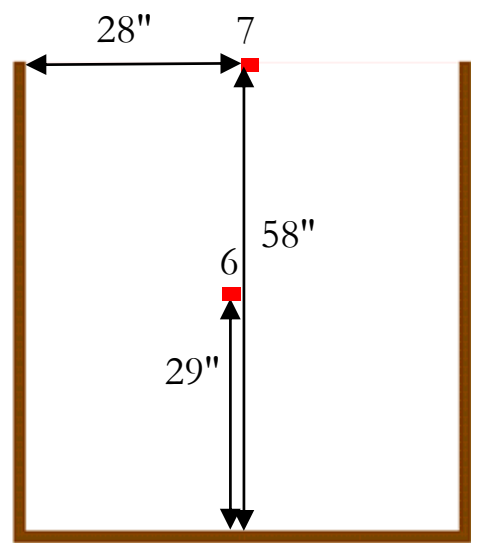

LS-3-GB-06: center (under cavity) LS-3-GB-07: foamy layer

(b)

Figure 6.2. Diagram of LS-3 Glass Block (a) with Sample Locations (b). 


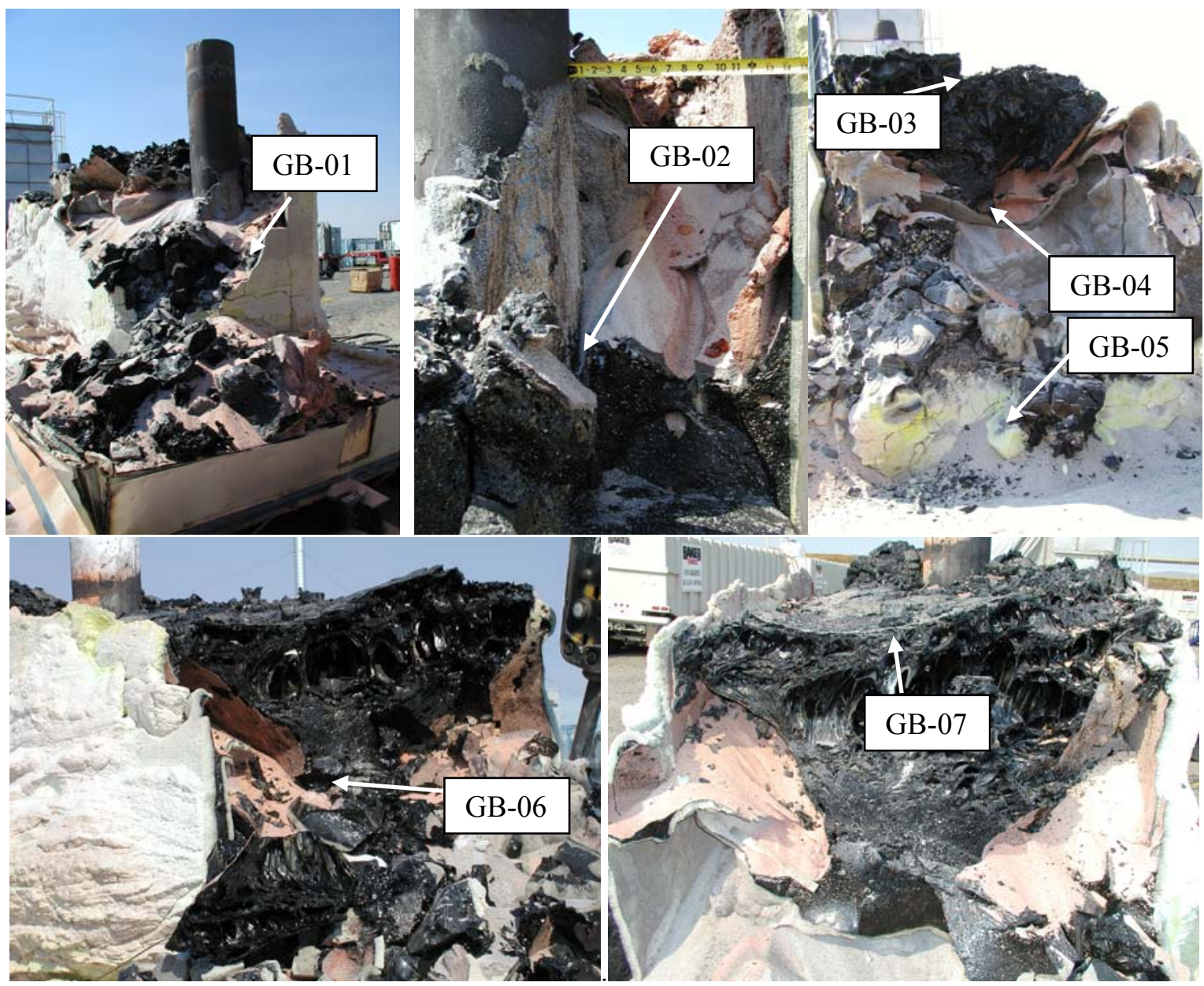

Figure 6.3. Photographs of LS-3 Block with Sample Locations Marked.

\subsection{Chemical Analysis}

Table 6.3 summarizes the results of glass-composition (Re that was not measured) and iron-redox analyses of the five samples (LS-3-02, -04, -05, -06, and -07) taken from different locations in the LS-2 glass block. Among these samples, glass composition was reasonably constant, which indicates that the glass melt was well mixed by convection currents during the melting process. Figure 6.4 compares of measured and target concentrations of seven major glass components (those with a target mass fraction higher than 0.03) in these samples. The Target/Combined column in 
Table 6.2 was used for target composition. Compared to target concentrations, measured values were higher for $\mathrm{SiO}_{2}$, similar for $\mathrm{CaO}$ and $\mathrm{Fe}_{2} \mathrm{O}_{3}$, and lower for the remeining the four components. The difference of measured versus target concentrations ranged from 20 to $23 \%$ for $\mathrm{SiO}_{2}$ and from 7 to $27 \%$ for the four major components that had lower measured concentrations than targets. As in LS-2, the increase of $\mathrm{SiO}_{2}$ resulted from the dissolution of silica sand that lined the container walls to insulate them from the glass melt. Table 6.4 lists the $S$ values obtained from Equation 5.1, using the analyzed compositions of sand and LS-3 glass samples in Table 4.1 and Table 6.3. Estimated $S$ ranged from 0.19 to 0.22 with an average of 0.20 . This estimated value is slightly larger than those obtained from LS-2 but still much smaller than those from engineering-scale tests. The average redox ratio of iron in glass was 0.45 , ranging from 0.41 to 0.52 , which was slightly lower than that for the LS-2 glasses.

Table 6.3. Measured Compositions of LS-3 Glass Samples (in mass fractions)

\begin{tabular}{|c|c|c|c|c|c|c|}
\hline Component & LS-3-02 & LS-3-04 & LS-3-05 & LS-3-06 & LS-3-07 & Average \\
\hline $\mathrm{Al}_{2} \mathrm{O}_{3}$ & 0.0848 & 0.0860 & 0.0874 & 0.0845 & 0.0853 & 0.0856 \\
\hline $\mathrm{B}_{2} \mathrm{O}_{3}$ & 0.0408 & 0.0399 & 0.0402 & 0.0391 & 0.0392 & 0.0398 \\
\hline $\mathrm{CaO}$ & 0.0289 & 0.0297 & 0.0303 & 0.0290 & 0.0294 & 0.0294 \\
\hline $\mathrm{Cr}_{2} \mathrm{O}_{3}$ & 0.0007 & 0.0007 & 0.0007 & 0.0007 & 0.0007 & 0.0007 \\
\hline $\mathrm{Fe}_{2} \mathrm{O}_{3}$ & 0.0442 & 0.0443 & 0.0455 & 0.0453 & 0.0440 & 0.0447 \\
\hline $\mathrm{K}_{2} \mathrm{O}$ & 0.0149 & 0.0154 & 0.0152 & 0.0150 & 0.0153 & 0.0152 \\
\hline $\mathrm{MgO}$ & 0.0135 & 0.0135 & 0.0140 & 0.0132 & 0.0134 & 0.0135 \\
\hline $\mathrm{Na}_{2} \mathrm{O}$ & 0.1239 & 0.1280 & 0.1277 & 0.1282 & 0.1300 & 0.1276 \\
\hline $\mathrm{P}_{2} \mathrm{O}_{5}$ & 0.0042 & 0.0041 & 0.0043 & 0.0041 & 0.0030 & 0.0039 \\
\hline $\mathrm{SiO}_{2}$ & 0.5913 & 0.5829 & 0.5758 & 0.5827 & 0.5912 & 0.5848 \\
\hline $\mathrm{SO}_{3}$ & 0.0009 & 0.0012 & 0.0009 & 0.0010 & 0.0013 & 0.0011 \\
\hline $\mathrm{TiO}_{2}$ & 0.0078 & 0.0078 & 0.0080 & 0.0076 & 0.0078 & 0.0078 \\
\hline $\mathrm{ZrO}_{2}$ & 0.0626 & 0.0649 & 0.0663 & 0.0618 & 0.0638 & 0.0639 \\
\hline Total & 1.0186 & 1.0183 & 1.0163 & 1.0122 & 1.0244 & 1.0180 \\
\hline \multicolumn{7}{|l|}{ Redox } \\
\hline $\mathrm{Fe}(\mathrm{II}) / \mathrm{Fe}($ total $)$ & 0.52 & 0.44 & 0.41 & 0.45 & 0.44 & 0.45 \\
\hline
\end{tabular}




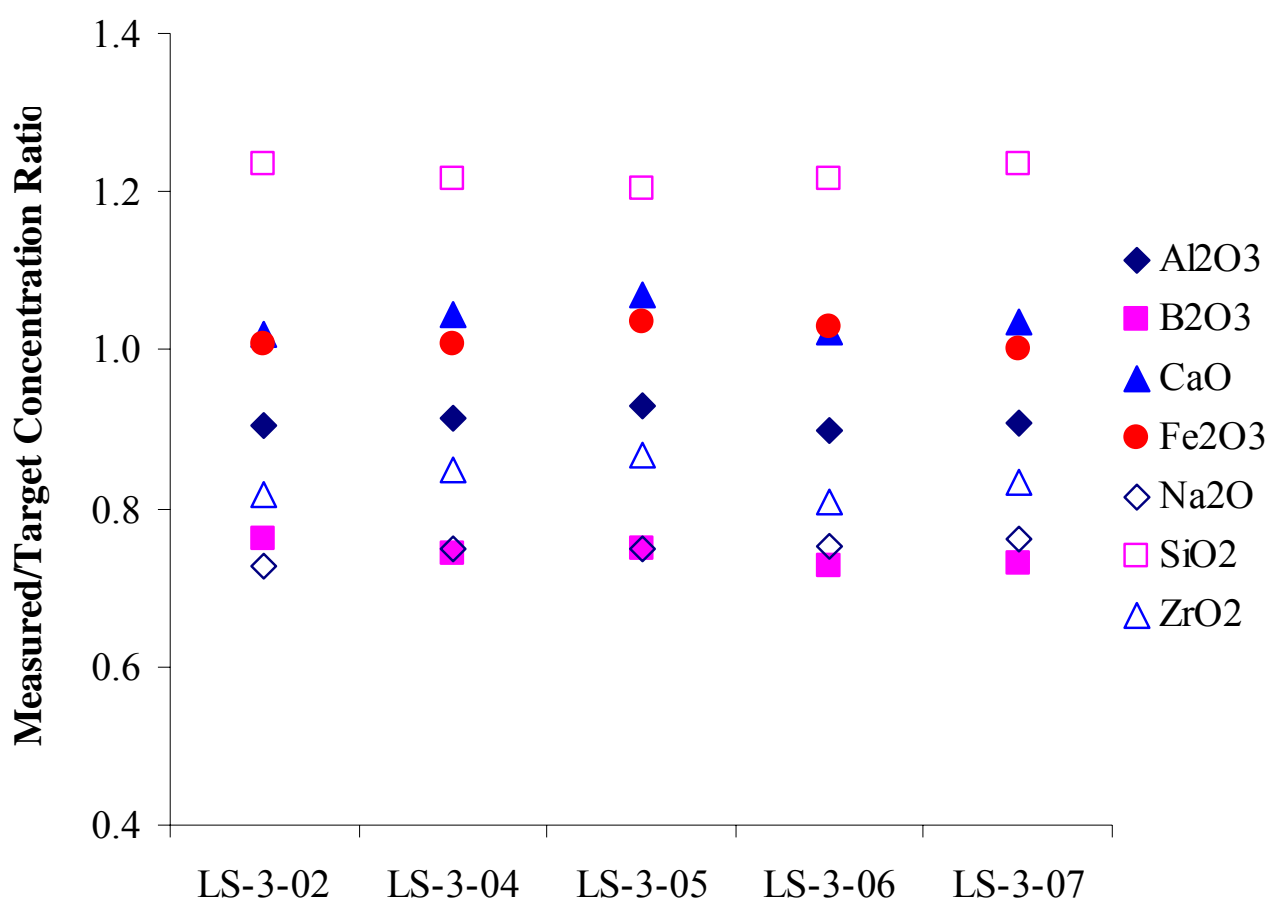

LS-2 Sample ID

Figure 6.4. Ratio of Measured and Target (Adjusted) Concentrations Selected Major Components in LS-3 Glasses

Table 6.4. Estimated Mass Fraction of Sand Dissolved in the LS-3 Glass Samples $(\mathcal{S}$ ) Calculated Based on Analyzed Glass Compositions

\begin{tabular}{||c|c|c|c|c|c|c||}
\hline Component & LS-3-02 & LS-3-04 & LS-3-05 & LS-3-06 & LS-3-07 & Average \\
\hline$S$ & 0.22 & 0.20 & 0.19 & 0.20 & 0.21 & 0.20 \\
\hline
\end{tabular}

\subsection{Product Consistency Test}

Table 6.5 summarizes the PCT response of LS-3 glass samples. The normalized releases were calculated based on the "Combined" target compositions in Table 6.2. The normalized releases from LS-3 glasses were much lower (roughly 1/3) than quenched and SC-treated ASCM-01 glasses that had similar target composition. The same trend was also observed in engineering-scale tests summarized in Section 3.3, which was attributed to the dissolution of silica sand. There was little difference between samples taken at different locations, suggesting that the glass was well homogenized during the melting process. The same conclusion was reached from the analyses of measured glass compositions (see Section 6.3). 
Table 6.5. PCT Responses for LS-3 Glasses

\begin{tabular}{||c|c|c|c|c|c||}
\hline \hline Component & LS-3-02 & LS-3-04 & LS-3-05 & LS-3-06 & LS-3-07 \\
\hline 7-day PCT leachate concentration (mg/L) \\
\hline$c_{N a}$ & 26.0 & 31.5 & 27.9 & 27.6 & 27.7 \\
\hline$c_{A l}$ & 4.7 & 5.8 & 5.1 & 4.8 & 4.9 \\
\hline$c_{B}$ & 2.7 & 2.9 & 2.6 & 2.4 & 2.4 \\
\hline$c_{S i}$ & 34.4 & 34.7 & 35.4 & 35.7 & 34.7 \\
\hline 7-day PCT Normalized release $\left(\mathrm{g} / \mathrm{m}^{2}\right)$ & \multicolumn{1}{l||}{} \\
\hline$r_{N a}$ & 0.103 & 0.124 & 0.110 & 0.109 & 0.109 \\
\hline$r_{A l}$ & 0.047 & 0.058 & 0.051 & 0.048 & 0.049 \\
\hline$r_{B}$ & 0.081 & 0.088 & 0.077 & 0.072 & 0.073 \\
\hline$r_{S i}$ & 0.077 & 0.078 & 0.079 & 0.080 & 0.078 \\
\hline \hline
\end{tabular}

\subsection{Vapor Hydration Test}

The VHT results of the glass samples taken from different positions of the LS-3 glass block after 14 days at $200^{\circ} \mathrm{C}$ are listed in Table 6.6. Within experimental uncertainty, the 14-day VHT responses of all these specimens are zero, suggesting a very durable glass product.

Table 6.6. Measured VHT Responses for LS-3 Specimens

\begin{tabular}{||l|c|c|c||}
\hline \hline Sample ID & $\mathrm{t}($ day $)$ & $\begin{array}{c}\mathrm{m}_{\mathrm{a}} \\
\left(\mathrm{g} / \mathrm{m}^{2}\right)^{(\mathrm{a})}\end{array}$ & $\mathrm{r}_{\mathrm{a}}\left(\mathrm{g} / \mathrm{m}^{2} / \mathrm{d}\right)$ \\
\hline LS-3-02-VHT-014 & 14.0 & -1 & -0.1 \\
\hline LS-3-04-VHT-014 & 14.0 & 4 & 0.3 \\
\hline LS-3-05-VHT-014 & 14.0 & -1 & -0.1 \\
\hline LS-3-06-VHT-014 & 14.0 & -2 & -0.2 \\
\hline LS-3-07-VHT-014 & 14.0 & -1 & -0.1 \\
\hline Average & 14.0 & -0.2 & 0.0 \\
\hline (a) Estimated measurement uncertainty is $\pm 10 \mathrm{~g} / \mathrm{m}^{2}$. \\
\hline
\end{tabular}

\subsection{Toxicity Characteristic Leach Procedure}

Table 6.7 lists the TCLP responses of the glass samples taken from different positions of the LS-3 glass block. Table 6.7 shows that the Cr release detected in only one sample is more than an order of magnitude lower than the UTS limit of $0.6 \mathrm{mg} / \mathrm{L}$. As in LS-2 experiment, the LS-3 glasses had slightly lower TCLP $r_{\mathrm{B}}$ values compared to the crucible melt glass with a similar composition (quenched and SC treated ASCM-01). This is consistent with the PCT results although the difference is smaller. As expected, the LS-3 results are comparable to engineering-scale test data summarized in Section 6.3. 
Table 6.7. TCLP Responses for LS-3 Glasses

\begin{tabular}{|c|c|c|c|c|c|}
\hline $\begin{array}{c}\text { Glass } \\
\text { Sample }\end{array}$ & LS-3-02 & LS-3-04 & LS-3-05 & LS-3-06 & LS-3-07 \\
\hline$c_{B}(\mathrm{mg} / \mathrm{L})$ & 0.18 & 0.25 & 0.35 & 0.29 & 0.42 \\
\hline$c_{C r}(\mathrm{mg} / \mathrm{L})$ & & & 0.044 & & \\
\hline$r_{B}(\mathrm{mg} / \mathrm{L})$ & 10.80 & 15.01 & 21.01 & 17.41 & 25.21 \\
\hline
\end{tabular}

\subsection{Glass Density}

Glass density was measured for the same set of samples characterized in previous tests (LS-3-01 through 07). The results are listed in Table 6.8. The density values range from 2.55 to $2.64 \mathrm{~g} / \mathrm{mL}$ with a mean of $2.59 \mathrm{~g} / \mathrm{mL}$ and a standard deviation of $0.03 \mathrm{~g} / \mathrm{mL}$.

Table 6.8. Measured Densities of LS-3 Glass Samples

\begin{tabular}{||l|c|c||}
\hline Sample ID & Mean Density $(\mathrm{g} / \mathrm{mL})$ & Standard Deviation $(\mathrm{g} / \mathrm{mL})$ \\
\hline LS-3-01-D & 2.5547 & 0.0007 \\
\hline LS-3-02-D & 2.5582 & 0.0006 \\
\hline LS-3-03-D & 2.5875 & 0.0003 \\
\hline LS-3-04-D & 2.6416 & 0.0010 \\
\hline LS-3-05-D & 2.6236 & 0.0004 \\
\hline LS-3-06-D & 2.5889 & 0.0008 \\
\hline LS-3-07-D & 2.5602 & 0.0006 \\
\hline Average & 2.5878 & 0.0339 \\
\hline
\end{tabular}

\subsection{Secondary Phase Identification}

Samples of glass were characterized for the amount and type of inclusions, such as crystalline material, metallic droplets, graphite, or bubbles that formed during the process or cooling. Figure 6.5 shows a portion of the LS-3 glass block close to the top area used for XRD analysis. The left side of Figure 6.5 is towards the container wall. The rectangular insert represents the area of sample collection. Table 6.9 summarizes the crystalline phase concentrations from the XRD analyses, which is plotted against sample locations in Figure 6.6. As seen in Table 6.9 and Figure 6.6, samples 09 and 10 that represent glass region have a small fraction of quartz phase only, which may be a result of inclusion of the sand particles. The sandy region close to the container wall (Samples 01 to 07) contains $\mathrm{SiO}_{2}$ polymorphs (quartz, tridymite, and cristobalite) in overall similar proportions whereas the sandy region close the cavity (Samples 12 and 13) has mainly quartz. It seems that the sandy region close to the wall was kept at a temperature range favorable for transformation of quartz sand to tridymite and cristobalite. A small fraction of anhydrite $\left(\mathrm{CaSO}_{4}\right)$ phase was identified throughout the samples except for the glass region (09 and 10). However, it would be necessary to use SEM/EDS on these samples to positively identify this 
sulfate phase, which was not attempted in the present study. A small fraction of zircon $\left(\mathrm{ZrSiO}_{4}\right)$ was identified only in the sandy region close the cavity (Samples 12 and 13).

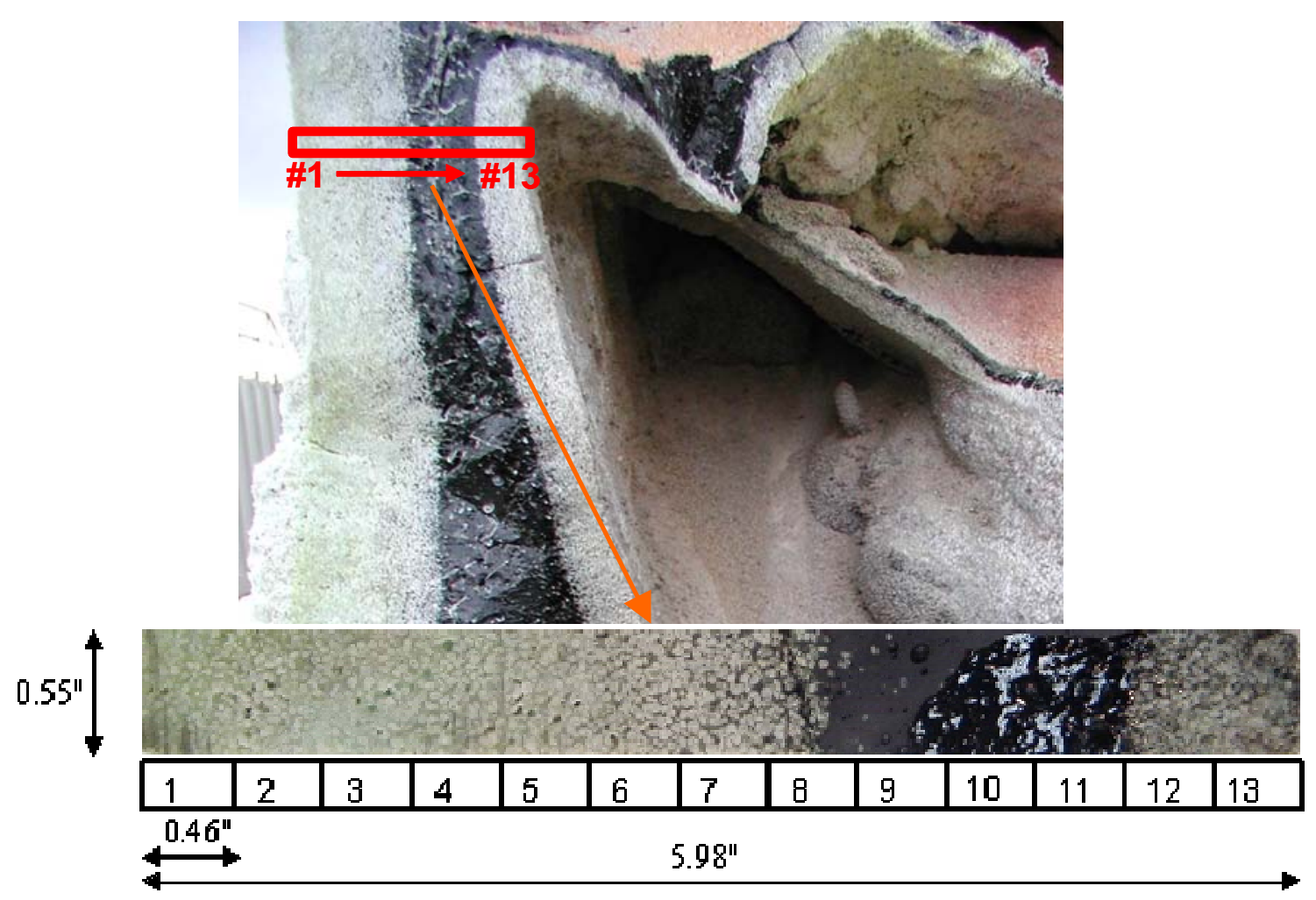

Figure 6.5. A Portion of LS-3 Glass Block Close to the Top Area Used for XRD Analysis

Table 6.9. Crystalline Phase Concentrations in LS-3 Glass Block Samples

\begin{tabular}{||c|c|c|c|c|c||}
\hline \hline & \multicolumn{5}{|c||}{ Phase concentrations in mass\% } \\
\hline Sample & $\begin{array}{c}\text { Quartz } \\
\left(\mathrm{SiO}_{2}\right)\end{array}$ & $\begin{array}{c}\text { Cristobalite } \\
\left(\mathrm{SiO}_{2}\right)\end{array}$ & $\begin{array}{c}\text { Tridymite } \\
\left(\mathrm{SiO}_{2}\right)\end{array}$ & $\begin{array}{c}\text { Anhydrite } \\
\left(\mathrm{CaSO}_{4}\right)\end{array}$ & $\begin{array}{c}\text { Zircon } \\
\left(\mathrm{ZrSiO}_{4}\right)\end{array}$ \\
\hline 01 & 15.1 & 4.8 & 13.4 & 1.4 & - \\
\hline 02 & 13.1 & 6.5 & 15.4 & 0.2 & - \\
\hline 03 & 10.6 & 8.8 & 16.6 & 0.1 & - \\
\hline 04 & 9.4 & 10.8 & 20.8 & 0.1 & - \\
\hline 05 & 10.2 & 12.4 & 20.8 & 0.3 & - \\
\hline 06 & 13.5 & 10.5 & 21.0 & 0.1 & - \\
\hline 07 & 20.9 & 8.6 & 15.8 & 0.8 & - \\
\hline 08 & 9.9 & 1.9 & - & 0.1 & - \\
\hline 09 & 0.6 & - & - & - & - \\
\hline 10 & 0.5 & - & - & - & - \\
\hline 11 & 9.2 & 2.5 & - & 0.2 & - \\
\hline 12 & 27.9 & 2.8 & - & 2.2 & 0.4 \\
\hline 13 & 38.0 & 4.9 & - & 2.3 & 0.6 \\
\hline
\end{tabular}




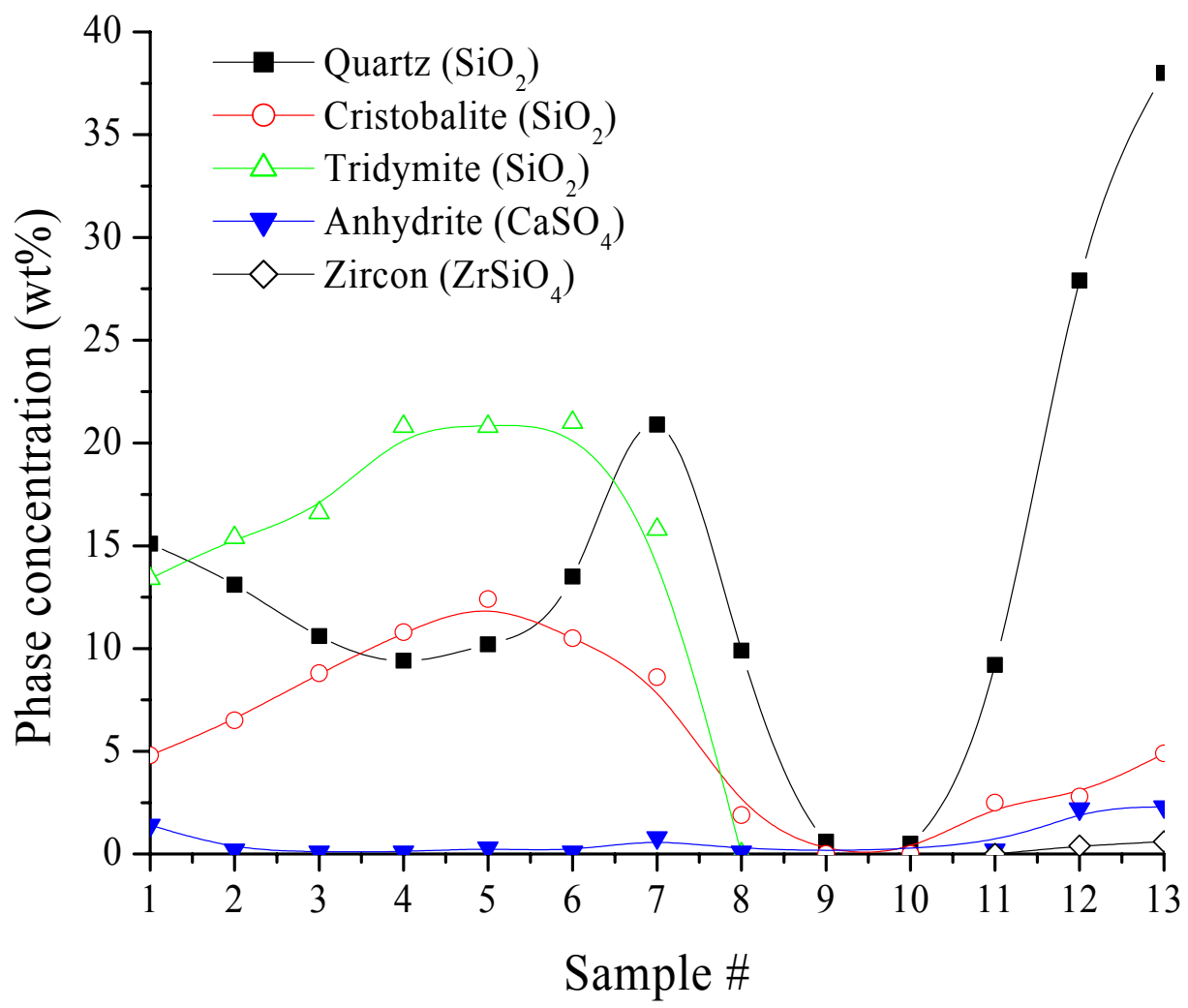

Figure 6.6. A Plot of Crystalline Phase Concentrations in LS-3 Glass Block Samples against Sample Location

In addition to XRD analyses above described, samples of sand layers from the LS-3 glass piece shown in Figure 6.5 were analyzed by ICP/AES. Figure 6.7 shows the representative location of sand layer samples taken for ICP analysis. Table 6.10 summarizes the measured compositions of four sand layer samples. Also included in Table 6.10 is the average composition of five glass samples in Table 6.3 for comparison. As expected, the relative concentrations of most components other than $\mathrm{SiO}_{2}$ in sand layers approximately resemble those in glass samples, suggesting the presence of glass phase between sand particles. One noticeable exception was $\mathrm{SO}_{3}$, which is higher in sand layer samples than in glass, especially in sample 12. Among three samples taken from the sand layers in the container wall side (09, 11, and 12), the glass fraction decreases (judging from $\mathrm{SiO}_{2}$ concentration) but the concentration of $\mathrm{SO}_{3}$ increases as it moves away from the glass interface. It is likely that the sulfate volatilized from glass melt condensed within the sand layers kept at relatively low temperatures. 


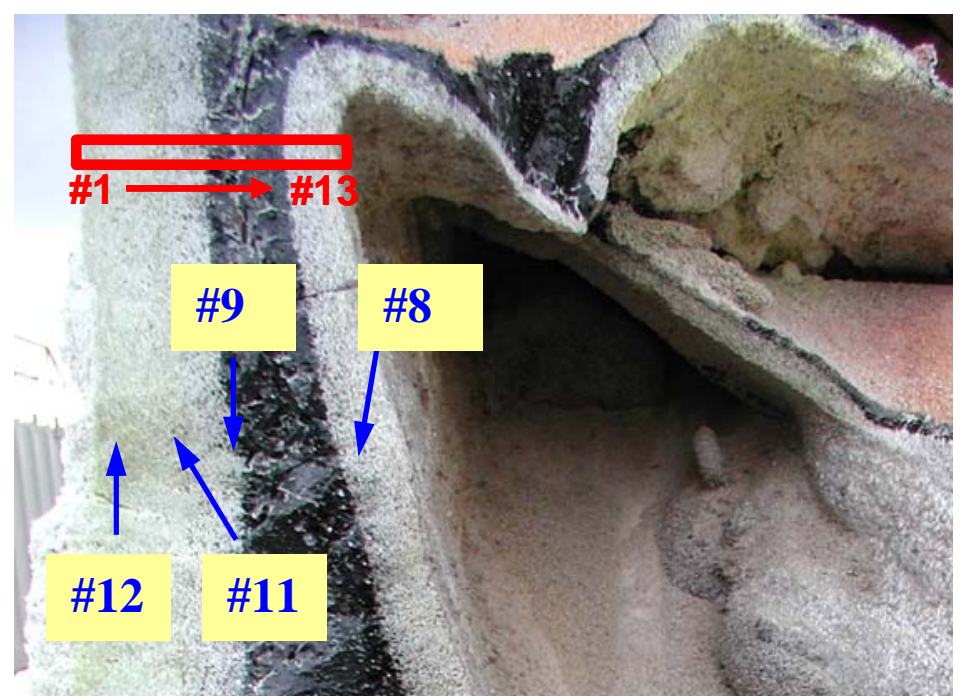

Figure 6.7. A Portion of LS-3 Glass Block Showing the Location of Sand Layer Samples for Composition Analyses

Table 6.10. Measured Compositions of Sand Layers in LS-3 Glass Block

\begin{tabular}{||l|c|c|c|c|c||}
\hline \hline \multicolumn{1}{|c|}{ Component } & $\begin{array}{c}\text { Average } \\
\text { in Glass Samples }\end{array}$ & $\mathbf{0 8}$ & $\mathbf{0 9}$ & $\mathbf{1 1}$ & $\mathbf{1 2}$ \\
\hline $\mathrm{Al}_{2} \mathrm{O}_{3}$ & 0.0856 & 0.0316 & 0.0364 & 0.0180 & 0.0045 \\
\hline $\mathrm{B}_{2} \mathrm{O}_{3}$ & 0.0398 & 0.0164 & 0.0169 & 0.0077 & 0.0013 \\
\hline $\mathrm{CaO}$ & 0.0294 & 0.0099 & 0.0129 & 0.0028 & 0.0000 \\
\hline $\mathrm{Cr}_{2} \mathrm{O}_{3}$ & 0.0007 & 0.0005 & 0.0005 & 0.0006 & 0.0005 \\
\hline $\mathrm{Fe}_{2} \mathrm{O}_{3}$ & 0.0447 & 0.0163 & 0.0177 & 0.0070 & 0.0021 \\
\hline $\mathrm{K}_{2} \mathrm{O}$ & 0.0152 & 0.0063 & 0.0066 & 0.0053 & 0.0014 \\
\hline $\mathrm{MgO}$ & 0.0135 & 0.0048 & 0.0054 & 0.0015 & 0.0000 \\
\hline $\mathrm{Na}_{2} \mathrm{O}$ & 0.1276 & 0.0458 & 0.0637 & 0.0645 & 0.0189 \\
\hline $\mathrm{P}_{2} \mathrm{O}_{5}$ & 0.0039 & 0.0015 & 0.0017 & 0.0010 & 0.0004 \\
\hline $\mathrm{SiO}_{2}$ & 0.5848 & 0.8587 & 0.8277 & 0.8819 & 0.9898 \\
\hline $\mathrm{SO}_{3}$ & 0.0011 & 0.0006 & 0.0020 & 0.0035 & 0.0050 \\
\hline $\mathrm{TiO}_{2}$ & 0.0078 & 0.0031 & 0.0034 & 0.0020 & 0.0005 \\
\hline $\mathrm{ZrO}_{2}$ & 0.0639 & 0.0214 & 0.0242 & 0.0093 & 0.0001 \\
\hline $\mathrm{Total}$ & & 1.0168 & 1.0191 & 1.0051 & 1.0245 \\
\hline \hline
\end{tabular}

Figure 6.8 shows the regions of LS-3 glass block samples used for SEM/EDS analyses. Region 1 is glass, region 2 is the interfacial area between the glass and sand layer, and regions 3 and 4 represent the sandy area of blue (region 3) and yellow (region 4) layers discussed in Section 4.2. A very small number of $\mathrm{ZrO}_{2}$ (that appears dissolving based on morphology) phases and zircon crystals were observed in regions 1 and 2. Figure 6.9 shows an example of these dissolving $\mathrm{ZrO}_{2}$ and crystal of zircon in region 1. An example of the interfacial region 2 is shown in Figure 6.10. Sand particles and crystals of $\mathrm{SiO}_{2}$ (presumably tridymite and cristobalite) that formed in the glassy area can be seen (darker phases in lighter background). Regions 3 and 4 are mainly sand particles with some glass phase in between. The inbetween glass phase typically contains a high fraction of $\mathrm{SiO}_{2}$ crystals (presumably tridymite and cristobalite) as illustrated in Figure 6.11. 


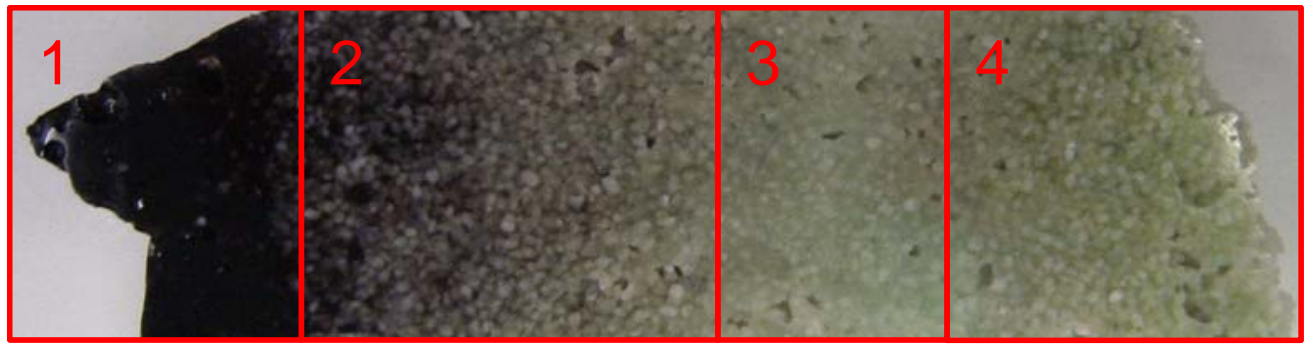

Figure 6.8. Regions of LS-3 Glass Block Used for SEM/EDS Analyses

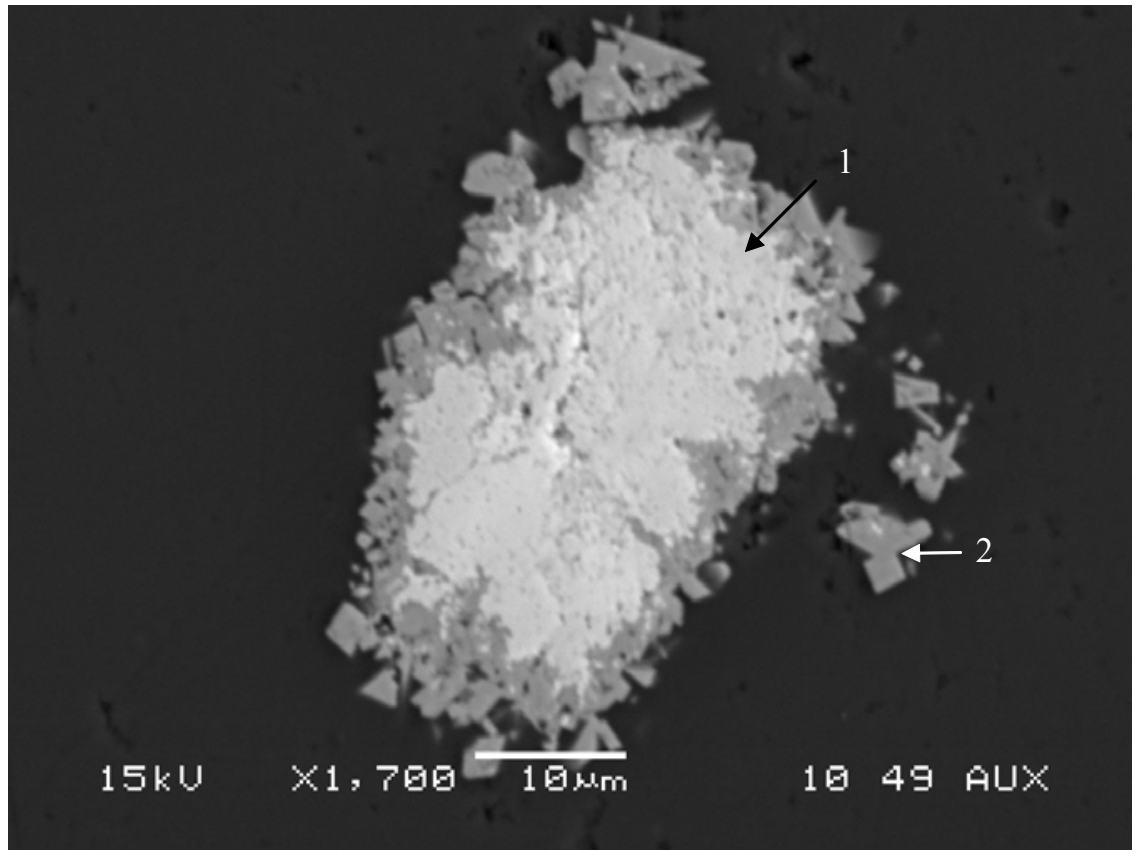

Figure 6.9. Dissolving $\mathrm{ZrO}_{2}$ (1) and Crystals of Zircon (2) Observed in Region 1 of LS-3 Glass Block Sample in Figure 6.8 


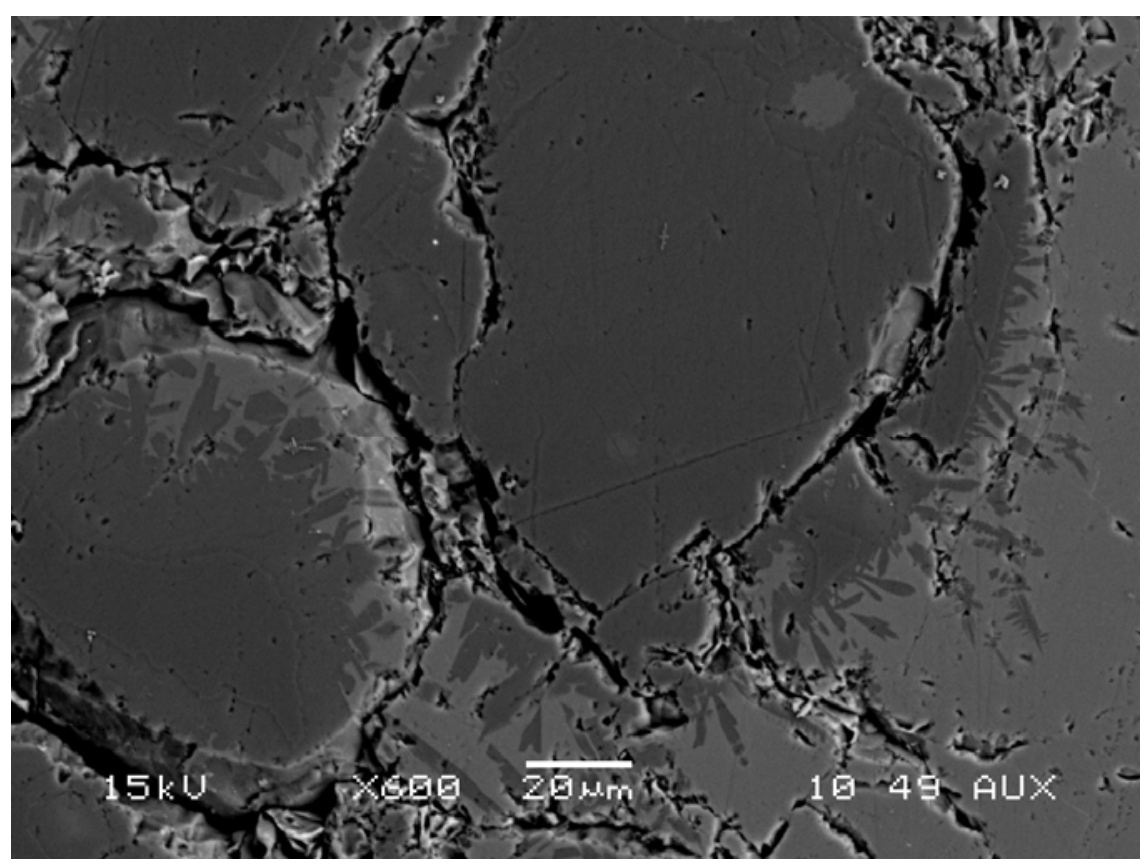

Figure 6.10. Sand Particles and Glassy Area with Crystals in Region 2 of LS-3 Glass Block Sample in Figure 6.8

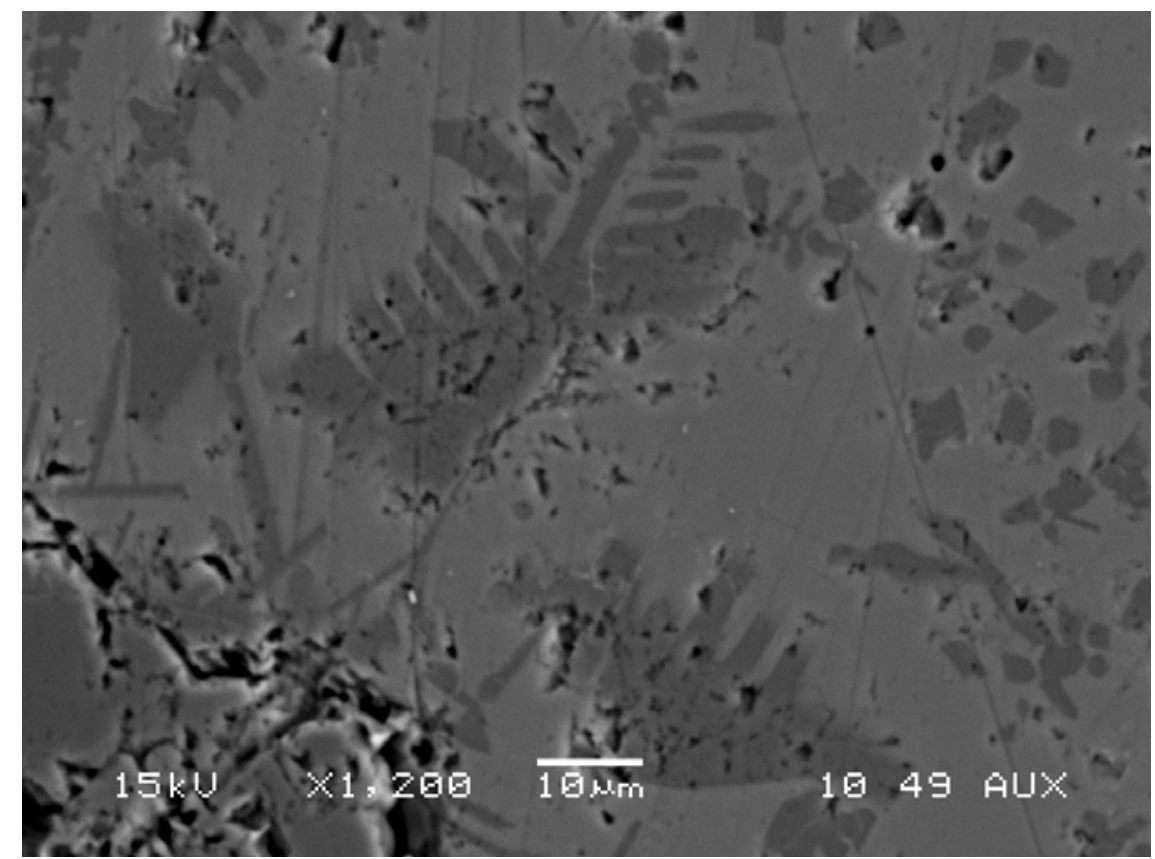

Figure 6.11. Crystals of $\mathrm{SiO}_{2}$ Polymorphs (Presumably Tridymite and Cristobalite) in Region 3 of LS-3 Glass Block Sample in Figure 6.8

\subsection{Summary and Conclusions}

This test has been designed to provide information necessary to assess the ICV process and product quality performance and to support full-scale engineering design. Glass was formulated with 19.7 mass $\%$ 
$\mathrm{Na}_{2} \mathrm{O}$. An extra amount of flux materials was added to the top of the dried feed. Assuming perfect mixing, the final target content of $\mathrm{Na}_{2} \mathrm{O}$ was 17.1 mass \%. Among five samples taken from different positions of the final block, glass composition was reasonably constant, indicating that the glass melt was well mixed. Due to the dissolution of silica sand that lined the container walls, measured concentrations of all components except $\mathrm{SiO}_{2}$ were lower than the targets. The average analyzed $\mathrm{Na}_{2} \mathrm{O}$ content in glass dropped to 12.8 mass $\%$. The average redox ratio of iron in glass was 0.45 , ranging from 0.41 to 0.52 .

The average normalized 7-day PCT Na release from LS-3 glasses was $0.11 \mathrm{~g} / \mathrm{m}^{2}$, and the TCLP Cr release was $0.04 \mathrm{mg} / \mathrm{L}$, detected in only one sample. The PCT and TCLP releases are more than an order of magnitude lower than the regulatory limits. The 14-day VHT responses of all specimens tested were zero, suggesting a very durable glass product. Glass density ranged from 2.55 to $2.64 \mathrm{~g} / \mathrm{mL}$ with a mean of $2.59 \mathrm{~g} / \mathrm{mL}$ and a standard deviation of $0.03 \mathrm{~g} / \mathrm{mL}$. SEM-EDS and XRD analysis of LS-3 glass block samples detected only very small fractions of quartz and zircon in the glass area. 


\subsection{References}

40 CFR 268.40. 2001. U.S. Environmental Protection Agency. "Applicability of Treatment Standards." (Land Disposal Restrictions). U.S. Code of Federal Regulations.

40 CFR 268.48. 2001. U.S. Environmental Protection Agency. "Universal Treatment Standards." (Land Disposal Restrictions). U.S. Code of Federal Regulations.

Allen, G. K., S. Blacker, R. Blackmon, P. J. Certa, W. Hamel, I. G. Papp, S. A. Saunders, K. R. Wells, and R. D. Williamson. 2002. River Protection Project System Plan, ORP-11242, Rev. 0, U. S.

Department of Energy, Office of River Protection, Richland, Washington.

AMEC. 2002. "Demonstration and Test Plan," in AMEC Proposal to Bulk Vitrification RFP \#93505, to CH2M Hill Hanford Group, November 2002.

American Society for Testing and Materials (ASTM). 1998. "Standard Test Methods for Determining Chemical Durability of Nuclear, Hazardous, and Mixed Waste Glasses: The Product Consistency Test (PCT)," C 1285-97 in 1998 Annual Book of ASTM Standards Vol. 12.01, ASTM, West Conshohocken, Pennsylvania.

American Society for Testing and Materials (ASTM). 1999. "Standard Test Methods for Electrical Conductivity and Resistivity of Water," ASTM D 1125-95 (Reapproved 1999), West Conshohocken, Pennsylvania.

Bechtel National, Inc. (BNI) 2003. Design, Construction, and Commissioning of the Hanford Tank Waste Treatment and Immobilization Plant Contract, DE-AC27-01RV14136, US Department of Energy, Office of River Protection, Richland, Washington.

Buelt, J. L., C. L. Timmerman, K. H. Oma, V. F. FitzPatrick, and J. G. Carter. 1987. In Situ Vitrification of Transuranic Waste: An Updated Systems Evaluation and Applications Assessment, PNL-4800, Suppl. 1, Pacific Northwest Laboratory, Richland, Washington.

Darab J. G., D. D. Graham, B. D. MacIsaac, R. L. Russell, D. K. Peeler, H. D. Smith, and J. D. Vienna. 2001. Sulfur Partitioning During Vitrification of INEEL Sodium Bearing Waste: Status Report. PNNL13588, Pacific Northwest National Laboratory, Richland, WA.

Ebert, W.L. and S.F. Wolf. 2000. "An Interlaboratory study of a standard glass for acceptance testing of low-activity waste glass,” J. Nuclear Materials, Vol. 282, 112-124.

EPA, see U.S. Environmental Protection Agency

Geosafe. 1998. Treatment Study for Planar In Situ Vitrification of INEEL Test Area North V-Tanks, INEEL/EXT-98-00854, Idaho National Engineering and Environmental Laboratory, Idaho Falls, Idaho.

Goles, R. W. 1996. Determination of Halogen Content in Glass for Assessment of Melter

Decontamination Factors, PNNL-11060, Pacific Northwest National Laboratory, Richland, Washington. 
Hrma P., G. F. Piepel, M. J. Schweiger, D. E. Smith, D-S. Kim, P. E. Redgate, J. D. Vienna, C. A. LoPresti, D. B. Simpson, D. K. Peeler, and M. H. Langowski. 1994. Property/Composition Relationships for Hanford High-Level Waste Glasses Melting at $1150^{\circ} \mathrm{C}$. PNL-10359, Vol. 1 and 2, Pacific Northwest Laboratory, Richland, WA.

Hrma, P., P. Izak, J.D. Vienna, G.M. Irwin and M-L. Thomas. 2002. "Partial Molar Liquidus Temperatures of Multivalent Elements in Multicomponent Borosilicate Glass." Phys. Chem. Glasses 43 (2) 128-136.

Jantzen, C. M., K. G. Brown, and J. B. Pickett. 2001. Impact of Phase Separation on Durability in Phosphate Containing Borosilicate Waste Glasses: Relevance to Vitrification of INEEL High Level Waste (U). WSRC-MS-2000-00307, Westinghouse Savannah River Company, Aiken, SC.

Kim, D-S. and J. D. Vienna. 2002. Model for TCLP Releases from Waste Glasses, PNNL-14061, Pacific Northwest National Laboratory, Richland, Washington.

Kot, W. K., and I. L. Pegg. 2001. Glass Formulation and Testing with RPP-WTP HLW Simulants - Final Report, VSL-01R2540-2, Vitreous State Laboratory, The Catholic University of America, Washington D.C.

Mann, F. M., K. C. Burgard, W. R. Root, R. J. Puigh, S. H. Finfrock, R. Khaleel, S. H. Bacon, E. J. Freeman, B. P. McGrail, S. K. Wurstner, and P. E. Lamont. 2001. Hanford Immobilized Low-Activity Waste Performance Assessment: 2001 Version, DOE/ORP-2000-24, Rev. 0, U. S. Department of Energy, Office of River Protection, Richland, Washington.

Muller, I. S., and I. L. Pegg. 1998. Glass Formulation and Testing with TWRS LAW Simulants, Final Report for GTS Durateck Inc. and BNFL Inc., Catholic University of America, Washington D.C.

Muller, I. S., A. C. Buechele, I. L. Pegg. 2001. Glass Formulation and Testing with RTP-WTP LAW Simulants-Final Report, VSL-01R3560-2, Vitreous State Laboratory at The Catholic University of America, Washington, D.C.

National Institute of Standards and Technology (NIST). 1980. Standard Reference Material 773, SodaLime-Silica Glass for Gradient-Furnace Liquidus Temperature. Washington, D.C.

Rapko, B. M., S. I. Sinkov, and T. G. Levitskaia. 2003. Removal of ${ }^{137}$ Cs from Dissolved Hanford Tank Saltcake by Treatment with IE-911, PNNL-14250, Pacific Northwest National Laboratory, Richland, Washington.

Rassat, S. D., L. A. Mahoney, R. L. Russell, S. A. Bryan, and R. L. Sell. 2003. Cold Dissolved Saltcake Waste Simulant Development, Preparation, and Analysis, PNNL-14194, Pacific Northwest National Laboratory, Richland, Washington.

Spalding, B. P., G. K. Jacobs, N. W. Dunbar, M. T. Naney, J. S. Tixier, and T. D. Powell. 1992. Tracerlevel Radioactive Pilot-scale Test of In Situ Vitrification for the Stabilization of Contaminated Soil Sites at ORNL, ORNL/TM-12201, Oak Ridge National Laboratory, Oak Ridge, Tennessee.

U.S. Environmental Protection Agency (EPA). 1992. Toxicity Characteristic Leach Procedure, SW-846 Method 1311, Washington, D. C. 
Vienna J. D., P. Hrma, A. Jiricka, D. E. Smith, T. H. Lorier, I. A. Reamer, and R. L. Schulz. 2001 Hanford Immobilized LAW Product Acceptance Testing: Tanks Focus Area Results, PNNL-13744, Pacific Northwest National Laboratory, Richland, Washington.

Vienna, J. D., D. S. Kim, and P. Hrma. 2002. Database and Interim Glass Property Models for Hanford HLW and LAW Glasses, PNNL-14060, Pacific Northwest National Laboratory, Richland, Washington.

Weier, D. R. and G. F. Piepel. 2003. Methodology for Adjusting and Normalizing Analyzed Glass Compositions, PNWD-3260 WTP-RPT-049, Rev. 0, Pacific Northwest National Laboratory, Richland, Washington.

Wolf, S. F., W. L. Ebert, J. S. Luo, and D. M. Strachan. 1998. "A Data Base and a Standard Material for Use in Acceptance Testing of Low-Activity Waste Products. ANL-98/9, Argonne National Laboratory, Argonne, IL. 
Appendix

\section{PNNL Technical Procedures Used}




\section{Appendix: PNNL Technical Procedures Used}

APEL-PAD-V, Rev. 2, Operation of Scintag Pad-V X-Ray Diffractometer, Safe Operating Procedure, 2002.

APEL-PIP-4, Rev. 2, Gas Pycnometry Method for Apparent Specific Gravity Determination of consolidated Solids, PNNL Technical Procedure, 2001.

GDL-ECC, Electrical Conductivity Calibration Procedure for Molten Glass, Pacific Northwest National Laboratory, Technical Procedure, 2003.

GDL-ELC, Electrical Conductivity Measurement Procedure, Pacific Northwest National Laboratory, Technical Procedure, 2003.

GDL-GBM, Rev. 3, Glass Batching and Melting, Pacific Northwest National Laboratory, Technical Procedure, 2002.

GDL-VHT, Vapor Hydration Test Procedure, Pacific Northwest National Laboratory, Technical Procedure, 2000.

GDL-VIS, Standard Viscosity Measurement Procedure for Vitrified Nuclear Waste, Pacific Northwest National Laboratory, Technical Procedure, 1998.

GDL-VSC, Standard Viscosity Calibration Procedure, Pacific Northwest National Laboratory, Technical Procedure, 1998.

GDL-XRD, Quantitative and Semi-quantitative analysis using X-Ray Diffraction, Pacific Northwest National Laboratory, Technical Procedure, 2002.

RPL-PIP-1, Rev.2, Preparation, Processing, and Testing of Radioactive Glass and Ceramics, PNNL Technical Procedure, 2001.

RPL-PIP-4, Rev. 2, Mounting Radioactive Samples in PIP XRD Sample Holder Base, Technical Procedure, 2002. 